\title{
New Islands of Tractability of Cost-Optimal Planning
}

\author{
Michael Katz, \\ Carmel Domshlak, \\ Faculty of Industrial Engineering and Management, \\ Technion - Israel Institute of Technology, Haifa, Israel
}

\author{
DUGI@TX.TECHNION.AC.IL, \\ DCARMEL@IE.TECHNION.AC.IL
}

\begin{abstract}
We study the complexity of cost-optimal classical planning over propositional state variables and unary-effect actions. We discover novel problem fragments for which such optimization is tractable, and identify certain conditions that differentiate between tractable and intractable problems. These results are based on exploiting both structural and syntactic characteristics of planning problems. Specifically, following Brafman and Domshlak (2003), we relate the complexity of planning and the topology of the causal graph. The main results correspond to tractability of cost-optimal planning for propositional problems with polytree causal graphs that either have $O(1)$-bounded in-degree, or are induced by actions having at most one prevail condition each. Almost all our tractability results are based on a constructive proof technique that connects between certain tools from planning and tractable constraint optimization, and we believe this technique is of interest on its own due to a clear evidence for its robustness.
\end{abstract}

\section{Précis}

AI problem solving is inherently facing a computational paradox. On the one hand, most general tasks of AI reasoning are known to be very hard, and this to a degree that membership in NP by itself is sometimes perceived as "good news". On the other hand, if the intelligence is somehow modeled by a computation, and the computation is delegated to computers, then artificial intelligence has to escape the traps of intractability as much as possible. Planning is one of such reasoning tasks, corresponding to finding a sequence of state-transforming actions that achieve a goal from a given initial state. It is well known that planning is intractable in general (Chapman, 1987), and that even the "simple" classical planning with propositional state variables is PSPACE-complete (Bylander, 1994).

While there were ups and downs in the interest of the planning community in the formal complexity analysis of planning problems, it is of a growing understanding these days that computational tractability is a fundamental issue in all problem solving. The pragmatic reasons for that are twofold.

1. Many planning problems in the manufacturing and other process controlling systems are believed to be highly structured, thus have a potential to allow for efficient planning if exploiting this structure (Klein, Jonsson, \& Bäckström, 1998). In fact, if this structure is not accounted for explicitly, a general-purpose planner is likely to go on tour in an exponential search space even for tractable problems. Moreover, since intractable theories provide no guarantees about the performance of engineering systems, in cases where such guarantees are required it is unavoidable to design 
the controlled system in a complexity-aware manner so that planning for it will be provably tractable (Williams \& Nayak, 1996, 1997).

2. Computational tractability can be an invaluable tool even for dealing with problems that fall outside all the known tractable fragments of planning. For instance, tractable fragments of planning provide the foundations for most (if not all) rigorous heuristic estimates employed in planning as heuristic search (Bonet \& Geffner, 2001; Hoffmann, 2003; Helmert, 2006; Hoffmann \& Nebel, 2001; Edelkamp, 2001). This is in particular true for admissible heuristic functions for planning that are typically defined as the optimal cost of achieving the goals in an over-approximating abstraction of the planning problem in hand. Such an abstraction is obtained by relaxing certain constraints in the specification of the original problem, and the purpose of the abstraction is to provide us with a provably tractable abstract problem (Haslum, 2006; Haslum \& Geffner, 2000; Haslum, Bonet, \& Geffner, 2005).

Unfortunately, the palette of known tractable fragments of planning is still very limited, and the situation is even more severe for tractable optimal planning. To our knowledge, just less than a handful of non-trivial fragments of optimal planning are known to be tractable. While there is no difference in theoretical complexity of regular and optimal planning in the general case (Bylander, 1994), many of the classical planning domains are provably easy to solve, but hard to solve optimally (Helmert, 2003). Practice also provides a clear evidence for strikingly different scalability of satisficing and optimal general-purpose planners (Hoffmann \& Edelkamp, 2005).

In this work we show that the search for new islands of tractability of optimal classical planning is far from being exhausted. Specifically, we study the complexity of optimal planning for problems specified in terms of propositional state variables, and actions that each changes the value of a single variable. In some sense, we continue the line of complexity analysis suggested by Brafman and Domshlak (2003), and extend it from satisficing to optimal planning. Our results for the first time provide a dividing line between tractable and intractable such problems.

\subsection{The UB (Optimal) Planning Problems}

Problems of classical planning correspond to reachability analysis in state models with deterministic actions and complete information. In this work we focus on state models describable in a certain fragment of the $\mathrm{SAS}^{+}$formalism (Bäckström \& Nebel, 1995) that allows only for propositional state variables and unary-effect actions. Following Bäckström and Nebel (1995), in what follows we refer to this subclass of $\mathrm{SAS}^{+}$as UB (short for "unary-effect, binary-valued"). Somewhat surprisingly, even non-optimal planning for UB is PSPACE-complete, that is, as hard as general propositional planning (Bylander, 1994).

Definition $1 A \mathbf{S A S}^{+}$problem instance is given by a quadruple $\Pi=\langle V, A, I, G\rangle$, where:

- $V=\left\{v_{1}, \ldots, v_{n}\right\}$ is a set of state variables, each associated with a finite domain $\operatorname{Dom}\left(v_{i}\right)$; the initial state $I$ is a complete assignment, and the goal $G$ is a partial assignment to $V$, respectively. 
- $A=\left\{a_{1}, \ldots, a_{N}\right\}$ is a finite set of actions, where each action a is a pair $\langle\operatorname{pre}(a), \operatorname{eff}(a)\rangle$ of partial assignments to $V$ called preconditions and effects, respectively. Each action $a \in A$ is associated with a non-negative real-valued cost $\mathcal{C}(a)$. An action a is applicable in a state $s \in \operatorname{Dom}(V)$ iff $s[v]=\operatorname{pre}(a)[v]$ whenever $\operatorname{pre}(a)[v]$ is specified. Applying an applicable action a changes the value of each variable $v$ to $\operatorname{eff}(a)[v]$ if $\operatorname{eff}(a)[v]$ is specified.

A $\mathrm{SAS}^{+}$problem instance belongs to the fragment $\mathrm{UB}$ of $\mathrm{SAS}^{+}$iff all the state variables in $V$ are binary-valued, and each action changes the value of exactly one variable, that is, for all $a \in A$, we have $|\operatorname{eff}(a)|=1$.

Different sub-fragments of UB can be defined by placing syntactic and structural restrictions on the actions sets of the problems. For instance, Bylander (1994) shows that planning in UB domains where each action is restricted to have only positive preconditions is tractable, yet optimal planning for this UB fragment is hard. In general, the seminal works by Bylander (1994) and Erol, Nau, and Subrahmanian (1995) indicate that extremely severe syntactic restrictions on single actions are required to guarantee tractability, or even membership in NP. Bäckström and Klein (1991) consider syntactic restrictions of a more global nature, and show that UB planning is tractable if no two actions have the same effect, and the preconditions of no two actions require different values for the variables that are not affected by these actions. Interestingly, this fragment of UB, known as PUBS, remains tractable for optimal planning as well. While the characterizing properties of PUBS are very restrictive, this result of Bäckström and Klein has provided an important milestone in the research on planning tractability.

Given the limitations of syntactic restrictions observed by Bylander (1994), Erol et al. (1995), and Bäckström and Klein (1991), more recent works have studied the impact of posing structural and mixed structural/syntactic restrictions on the action sets. In the scope of UB, most of these works relate the complexity of planning and the topological properties of the problem's causal graph structure.

Definition 2 The causal graph $C G(\Pi)$ of a $\mathrm{SAS}^{+}$problem $\Pi=\langle V, A, I, G\rangle$ is a digraph over the nodes $V$. An arc $\left(v, v^{\prime}\right)$ belongs to $C G(\Pi)$ iff $v \neq v^{\prime}$ and there exists an action $a \in A$ changing the value of $v^{\prime}$ while being preconditioned by some value of $v$, that is, both $\operatorname{eff}(a)\left[v^{\prime}\right]$ and $\operatorname{pre}(a)[v]$ are specified.

Informally, the immediate predecessors of $v$ in $C G(\Pi)$ are all those variables that directly affect our ability to change the value of $v$, and it is evident that constructing the causal graph $C G(\Pi)$ of any given UB planning problem $\Pi$ is straightforward. For instance, consider the action set depicted in Figure 1a. It is easy to verify that all the actions in this set are unary effect. The causal graph induced by this action set is depicted in Figure 1b. The actions $a_{1}$ and $a_{2}$ are the only actions that change the values of $v_{1}$ and $v_{2}$, respectively, and these actions have no preconditions outside the affected variables. Hence, the causal graph contains no arcs incoming to the nodes $v_{1}$ and $v_{2}$. On the other hand, the actions changing $v_{3}$ and $v_{4}$ are preconditioned (in both cases) by the values of $v_{1}$ and $v_{2}$, and thus both $v_{3}$ and $v_{4}$ have incoming arcs from $v_{1}$ and $v_{2}$.

Way before being used for the complexity analysis, causal graphs have been (sometimes indirectly) considered in the scope of hierarchically decomposing planning tasks (Newell \& 


\begin{tabular}{|c||c|c|c|c||c|c|c|c|}
\hline \multicolumn{1}{|c||}{$A$} & \multicolumn{5}{c||}{$\operatorname{pre}(a)$} & \multicolumn{4}{c|}{$\operatorname{eff}(a)$} \\
\hline & $v_{1}$ & $v_{2}$ & $v_{3}$ & $v_{4}$ & $v_{1}$ & $v_{2}$ & $v_{3}$ & $v_{4}$ \\
\hline \hline$a_{1}$ & 0 & & & & 1 & & & \\
\hline$a_{2}$ & & & & & & 1 & & \\
\hline$a_{3}$ & 0 & 0 & & & & & 1 & \\
\hline$a_{4}$ & 0 & 0 & & 0 & & & & 1 \\
\hline$a_{5}$ & & 1 & & 1 & & & & 0 \\
\hline \hline
\end{tabular}

(a)

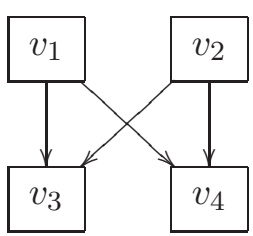

(b)

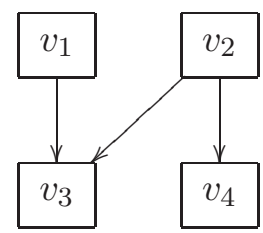

(c)

Figure 1: Example of two simple action sets that fit the characteristics of the UB fragment. (a) Unary-effect action set $A$ over propositional variables $V=\left\{v_{1}, \ldots, v_{4}\right\}$, (b) Causal graph induced by $A$, (c) Causal graph induced by $A \backslash\left\{a_{4}\right\}$.

Simon, 1963; Sacerdoti, 1974; Knoblock, 1994; Tenenberg, 1991; Bacchus \& Yang, 1994). The first result relating between the complexity of UB planning and the structure of the causal graph is due to Bäckström and Jonsson (1995, 1998b) that identify a fragment of $\mathrm{UB}$, called $\mathbf{3 S}$, which has an interesting property of inducing tractable plan existence yet intractable plan generation. One of the key characteristics of $\mathbf{3 S}$ is the acyclicity of the causal graphs. A special case of $\mathbf{3 S}$ is also independently studied by Williams and Nayak (1997) in the scope of incremental planning for more general $\mathrm{SAS}^{+}$problems.

More recently, Brafman and Domshlak (2003) provide a detailed account of the complexity of finding plans for UB problems with acyclic causal graphs. These results are most closely related to the problems examined in our paper, and thus we survey them in more details. For ease of presentation, we now introduce certain notation that is heavily used throughout the paper.

- For each node $v \in C G(\Pi)$, by $\ln (v)$ and $\operatorname{Out}(v)$ we denote the in- and out-degrees of $v$, respectively, and $\ln (C G(\Pi)) /$ Out $(C G(\Pi))$ stand for the maximal in-degree/out-degree of the $C G(\Pi)$ nodes.

- Assuming $C G(\Pi)$ is connected ${ }^{1}$, we provide a special notation to the following topologies of acyclic causal graphs, also depicted in Figure 2. A causal $C G(\Pi)$ is a

$\mathbf{T}$ tree if $\ln (C G(\Pi)) \leq 1$, and there exists $v \in V$ such that $\ln (v)=0$.

I inverted tree if Out $(C G(\Pi)) \leq 1$, and there exists $v \in V$ such that Out $(v)=0$.

$\mathbf{P}$ polytree if $C G(\Pi)$ contains no undirected cycles. (For an example of a polytree that is neither tree nor inverted tree see Figure 1c or Figure 2.)

$\mathbf{S}$ directed-path singly connected if there is at most one directed path from each node $v \in C G(\Pi)$ to any other node $v^{\prime} \in C G(\Pi)$. (For an example of a directed-path singly connected DAG see Figure $1 \mathrm{~b}$ or Figure 2.)

1. If $C G(\Pi)$ consists of a few connected components, then these components identify independent subproblems of $\Pi$ that can be easily identified and treated separately. 


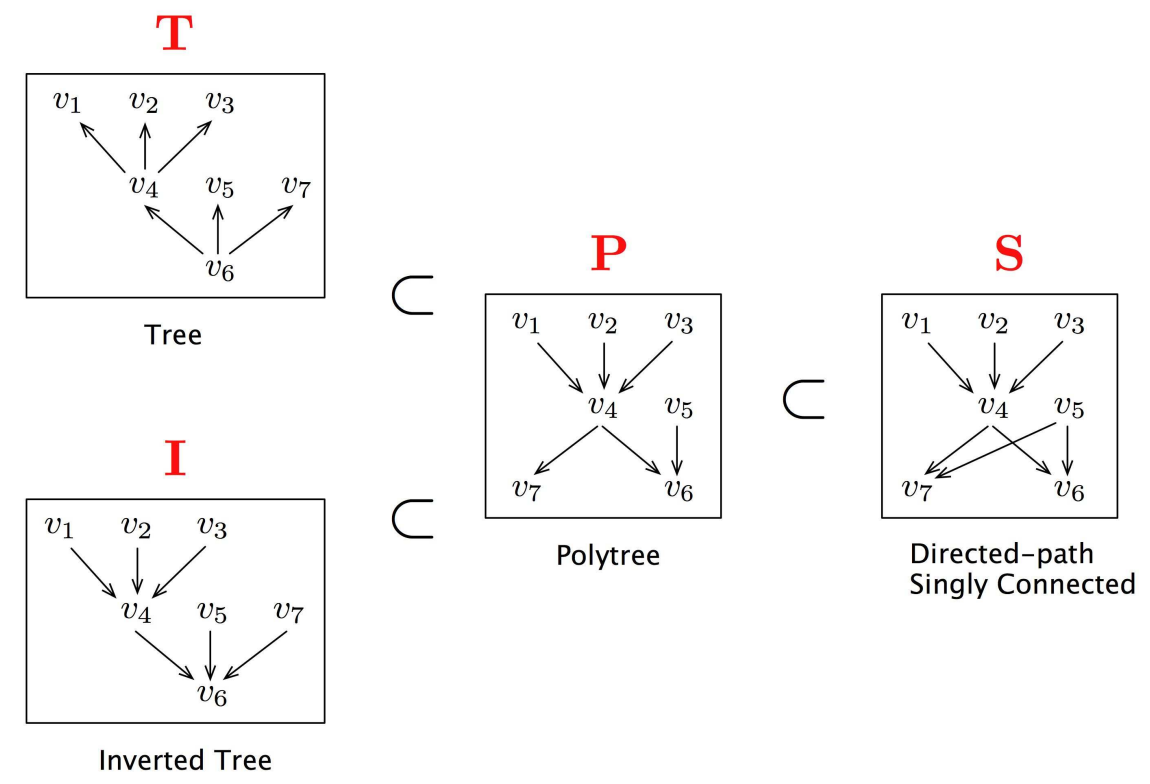

Figure 2: Examples of the causal graphs topologies considered in the paper, along with the inclusion relations between the induced fragments of UB.

In what follows, we use $\mathbf{T}, \mathbf{I}, \mathbf{P}$, and $\mathbf{S}$ to refer to the corresponding fragments of UB, and we use subscript/superscript $b$ to refer to a fragment induced by the additional constraint of in-degree/out-degree being bounded by a constant. It is not hard to verify that we have $\mathbf{T}, \mathbf{I} \subset \mathbf{P} \subset \mathbf{S}$, with $\mathbf{T} \subset \mathbf{P}_{b}$ and $\mathbf{I} \subset \mathbf{P}^{b}$; the complete inclusion hierarchy of these sub-fragments of UB is shown in Figure 3a.

The key tractability result by Brafman and Domshlak (2003) corresponds to a polynomial time plan generation procedure for $\mathbf{P}_{b}$, that is, for UB problems inducing polytree causal graphs with all nodes having $O(1)$-bounded indegree. In addition, Brafman and Domshlak show that plan generation is NP-complete for the fragment $\mathbf{S}$, and we note that their proof of this claim can be easily modified to hold for $\mathbf{S}_{b}^{b}$. These results of tractability and hardness (as well as their immediate implications) are depicted in Figure $3 \mathrm{~b}$ by the shaded bottom-most and the transparent top-most free-shaped regions. The empty freeshaped region in between corresponds to the gap left by Brafman and Domshlak (2003). This gap has been recently closed by Gimenez and Jonsson (2008) that prove NP-completeness of plan generation for $\mathbf{P}$. We note that the proof of Gimenez and Jonsson actually carries out to the I fragment as well, and so the gap left by Brafman and Domshlak (2003) is now entirely closed.

\subsection{Summary of Results}

The complexity results by both Brafman and Domshlak (2003) and Gimenez and Jonsson (2008) correspond to satisficing planning, and do not distinguish between the plans on the 


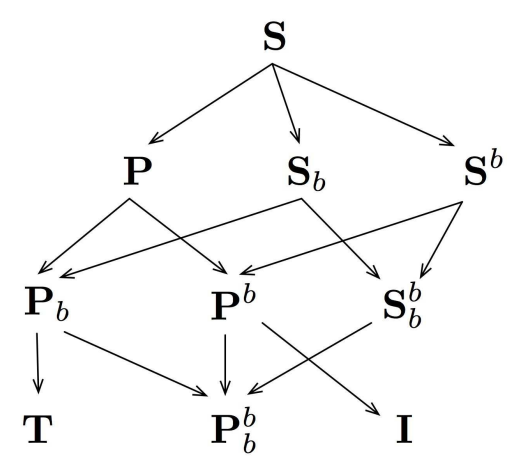

(a)

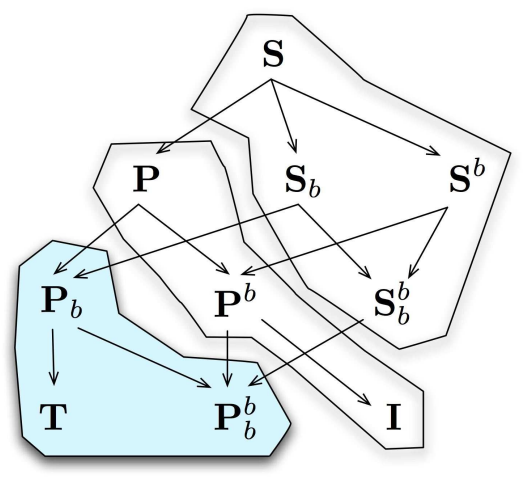

(b)

Figure 3: Inclusion-based hierarchy and complexity of plan generation for some UB problems with acyclic causal graphs. (a) The hierarchy of STRIPS fragments corresponding to tree, inverted tree, polytree, and directed-path singly connected topologies of the causal graph, and (possibly) $O(1)$ bounds on the causal graph in-degree and/or out-degree. (b) Plan generation is tractable for the fragments in the (bottom-most) shaded region, and NP-complete for all other depicted fragments. The top-most and intermediate (transparent) regions correspond to the results by Brafman and Domshlak (2003) and Gimenez and Jonsson (2008), respectively.

basis of their quality. In contrast, we study the complexity of optimal plan generation for UB, focusing on (probably the most canonical) cost-optimal (also known as sequentiallyoptimal) planning. Cost-optimal planning corresponds to the task of finding a plan $\rho \in A^{*}$ that minimizes $\mathcal{C}(\rho)=\sum_{a \in \rho} \mathcal{C}(a)$. We provide novel tractability results for cost-optimal planning for $\mathrm{UB}$, and draw a dividing line between the tractable and intractable such problems. Almost all our tractability results are based on a proof technique that connects between certain tools from planning and tractable constraint optimization. We strongly believe that this "proof-technical" contribution of the paper is of interest on its own due to a clear evidence for its robustness - our different algorithms exploit this proof technique, but in very much different manners.

In the rest of this section we aim at providing an adequate description of our results for readers who do not want to delve into formal details, or just prefer not to do it in the first reading of the paper. ${ }^{2}$ Hence, most formal definitions, constructions, and proofs underlying these results are given later, starting in Section 2.

2. We adopted this format from the seminal paper by Bylander (1994) as we feel the format has contributed something to making that paper an extremely enjoyable read. 


\subsubsection{Cost-Optimal Planning for $\mathbf{P}_{b}$}

Following Brafman and Domshlak (2003), we relate the complexity of (cost-optimal) UB planning and the topology of the causal graph. For that we consider the structural hierarchy depicted in Figure 3a. We begin with considering cost-optimal planning for $\mathbf{P}_{b}$-it is apparent from Figure $3 \mathrm{~b}$ that this is the most expressive fragment of the hierarchy that is still a candidate for tractable cost-optimal planning. Our first positive result affirms this possibility, showing that the complexity map of the cost-optimal planning for the UB fragments in Figure 3a is identical to this for satisficing planning (that is, Figure 3b).

Our algorithm for $\mathbf{P}_{b}$ is based on compiling a given $\mathbf{P}_{b}$ problem $\Pi$ into a constraint optimization problem $\mathrm{COP}_{\Pi}=(\mathcal{X}, \mathcal{F})$ over variables $\mathcal{X}$, functional components $\mathcal{F}$, and the global objective $\min \sum_{\varphi \in \mathcal{F}} \varphi(\mathcal{X})$ such that

(I) $\mathrm{COP}_{\Pi}$ can be constructed in time polynomial in the description size of $\Pi$,

(II) the tree-width of the cost network of $\mathrm{COP}_{\Pi}$ is bounded by a constant, and the optimal tree-decomposition of the network is given by the compilation process,

(III) if $\Pi$ is unsolvable then all the assignments to $\mathcal{X}$ evaluate the objective function to $\infty$, and otherwise, the optimum of the global objective is obtained on and only on the assignments to $\mathcal{X}$ that correspond to cost-optimal plans for $\Pi$,

(IV) given an optimal solution to $\mathrm{COP}_{\Pi}$, the corresponding cost-optimal plan for $\Pi$ can be reconstructed from the former in polynomial time.

Having such a compilation scheme, we then solve $\mathrm{COP}_{\Pi}$ using the standard, poly-time algorithm for constraint optimization over trees (Dechter, 2003), and find an optimal solution for $\Pi$. The compilation is based on a certain property of the cost-optimal plans for $\mathbf{P}_{b}$ that allows for conveniently bounding the number of times each state variable changes its value along such an optimal plan. Given this property of $\mathbf{P}_{b}$, each state variable $v$ is compiled into a single COP variable $x_{v}$, and the domain of that $\mathrm{COP}$ variable corresponds to all possible sequences of value changes that $v$ may undergo along a cost-optimal plan. The functional components $\mathcal{F}$ are then defined one for each COP variable $x_{v}$, and the scope of such a function captures the "family" of the original state variable $v$ in the causal graph, that is, $v$ itself and its immediate predecessors in $C G(\Pi)$. For an illustration, Figure 4a depicts a causal graph of a $\mathbf{P}$ problem $\Pi$, with the family of the state variable $v_{4}$ being depicted by the shaded region, and Figure $4 \mathrm{~b}$ shows the cost network induced by compiling $\Pi$ as a $\mathbf{P}_{b}$ problem, with the dashed line surrounding the scope of the functional component induced by the family of $v_{4}$. It is not hard to verify that such a cost network induces a tree over variable-families cliques, and for a $\mathbf{P}_{b}$ problem, the size of each such clique is bounded by a constant. Hence, the tree-width of the cost-network is bounded by a constant as well.

\subsubsection{Causal Graphs and $k$-Dependence}

While causal graphs provide important information about the structure of the planning problems, a closer look at their definition reveals that some information used for defining causal graphs actually gets hidden by this structure. To start with an example, let us consider the multi-valued encoding of the Logistics-style problems (Helmert, 2006). In 
these problems, each variable representing the location of a package has as its parents in the causal graph all the variables representing alternative transportation means (i.e., tracks, planes, etc.), and yet, each individual action affecting the location of a package is preconditioned by at most one such parent variable. (You cannot load/unload a package into/from more than one vehicle.) This exemplifies the fact that, even if the in-degree of the causal graph is proportional to some problem domain's parameters, the number of variables that determine applicability of each action may still be bounded by a constant.

In other words, while the causal graph provides an aggregate view on the independence relationships between the problem variables, the individual dependencies of the problem actions on the unaffected variables are suppressed by this view. Targeting these actual individual dependencies of the actions, here we define a (tangential to the causal graph's topology) classification of the UB problems, and study the connection between this classification and the computational tractability of both general and cost-optimal plan generation for UB.

Definition 3 For any $k \in \mathbb{Z}^{*}$, and any $\mathrm{SAS}^{+}$problem instance $\Pi=(V, A, I, G)$, we say that $\Pi$ is $\mathbf{k - d e p e n d e n t ~ i f ~ i t ~ s a t i s f i e s ~}$

$$
\max _{a \in A}|\{v \in V \mid \operatorname{pre}(a)[v] \neq \mathrm{u} \wedge \operatorname{eff}(a)[v]=\mathrm{u}\}| \leq k
$$

with "= u" standing for "unspecified".

In other words, a $\mathrm{SAS}^{+}$problem is $k$-dependent if no action in its action set depends on more than $k$ unaffected variables. Combining our two classifications of the problems, for any structural fragment $\mathbf{F}$ of UB (such as, e.g., these in Figure 3), and any $k \in \mathbb{Z}^{*}$, by $\mathbf{F}(k)$ we denote the set of all $k$-dependent problems within $\mathbf{F}$.

Recall that the fragment $\mathbf{P}$ of UB is NP-hard even for satisficing planning (Gimenez \& Jonsson, 2008). Our main result is positive - at least for the most extreme (yet, says the Logistics example above, not unrealistic) setting of $k=1$, satisfying $k$-dependence does bring us to an island of tractability $\mathbf{P}(1)$.

Similarly to our treatment of $\mathbf{P}_{b}$, our algorithm for $\mathbf{P}(1)$ exploits the idea of compiling a planning problem $\Pi$ into a tractable constraint optimization problem $\mathrm{COP}_{\Pi}$. However, the planning-to-COP compilation scheme for $\mathbf{P}(1)$ is very much different from that devised for $\mathbf{P}_{b}$. In fact, this difference is unavoidable since our construction for $\mathbf{P}_{b}$ heavily relies on the assumption that $\ln (C G(\Pi))=O(1)$, and we do not have this luxury in $\mathbf{P}(1)$. Instead, we identify certain properties of the cost-optimal plan sets of the $\mathbf{P}(1)$ problems, and exploit these properties in devising suitable planning-to-COP compilation schemes.

We begin with considering only $\mathbf{P}(1)$ problems with uniform-cost actions; the cost of a plan for such a problem is proportional to the length of the plan ${ }^{3}$. We show that any such solvable problem has a cost-optimal plan that makes all the changes of each variable to a certain value using exactly the same (type of) action. And while devising a correct and tractable planning-to-COP compilation scheme is more than a step away from identifying this property of $\mathbf{P}(1)$, the latter provides a critical brick that everything else relies upon. Relying on this property of $\mathbf{P}(1)$, each state variable $v$ and each edge $\left(v, v^{\prime}\right)$ are uniquely

3. This is probably the origin for the term "sequential optimality". 


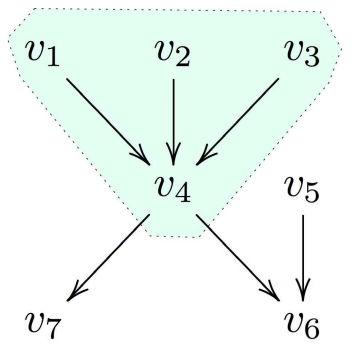

(a)

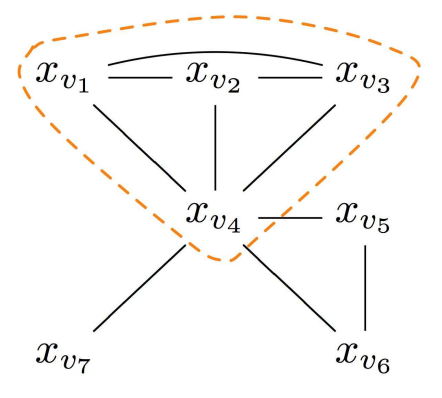

(b)

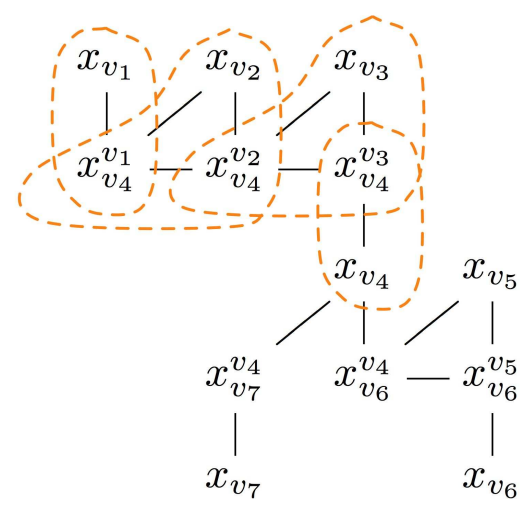

(c)

Figure 4: Cost networks induced by the planning-to-COP compilation schemes for $\mathbf{P}$. (a) Causal graph of a $\mathbf{P}$ problem $\Pi$, with the family of the state variable $v_{4}$ being depicted by the shaded region. (b) Cost network induced by compiling $\Pi$ as a $\mathbf{P}_{b}$ problem, with the dashed line surrounding the scope of the functional component induced by the family of $v_{4}$. (c) Cost network induced by compiling $\Pi$ as a $\mathbf{P}(1)$ problem, with the dashed lines surrounding the scopes of the four functional components induced by the family of $v_{4}$.

compiled into COP variables $x_{v}$ and $x_{v^{\prime}}^{v}$ (see Figure 4c). A certain set of functional components are then defined for each COP variable $x_{v}$. Both the domains of the COP variables and the specification of the functional components are technically involved, and thus relegated to later in the paper. It is important, however, to note already here that the cost networks of such COPs are guaranteed to induce trees over cliques of size $\leq 3$, and thus having tree-width bounded by a constant. The reader can get an intuition of where these "cliques of size $\leq 3$ " are coming from by looking on the example depicted in Figure 4c.

Unfortunately, the aforementioned helpful property of the $\mathbf{P}(1)$ problems with uniformcost actions does not hold for more general action-cost schemes for $\mathbf{P}(1)$. Turns out, however, that all problems in $\mathbf{P}(1)$ satisfy another property that still allows for devising a general, correct, and tractable planning-to-COP scheme for $\mathbf{P}(1)$. Specifically, we show that any solvable problem in $\mathbf{P}(1)$ has a cost-optimal plan that makes all the changes of each variable using at most three types of action. The algorithm resulting from exploiting this property is more complex and more costly than that devised for the $\mathbf{P}(1)$ problems with uniform-cost actions, yet it is still poly-time. Interestingly, the cost networks of such COPs are topologically identical to these for problems with uniform-cost actions, with the difference being in the domains of the COP variables, and in the specification of the functional components.

Having read this far, the reader may rightfully wonder whether $O(1)$-dependence is not a strong enough property to make the cost-optimal planning tractable even for some more complex than polytree forms of the causal graph. Turns out that the dividing line between 


\begin{tabular}{|l|c|c|c|c|}
\hline & $k=1$ & $k=2$ & $k=3$ & $k=\Theta(n)$ \\
\hline \hline $\mathbf{P}_{b}$ & - & - & - & $\mathrm{P}$ \\
\hline $\mathbf{P}(k)$ & $\mathrm{P}$ & & & $\mathrm{NPC}$ \\
\hline $\mathbf{S}_{b}^{b}$ & $\mathrm{NPC}$ & - & - & $\mathrm{NPC}$ \\
\hline
\end{tabular}

(a)

\begin{tabular}{|l|c|c|c|c|}
\hline & $k=1$ & $k=2$ & $k=3$ & $k=\Theta(n)$ \\
\hline \hline $\mathbf{P}_{b}$ & - & - & - & $\mathrm{P}$ \\
\hline $\mathbf{P}(k)$ & $\mathrm{P}$ & & & $\mathrm{NPC}$ \\
\hline $\mathbf{S}_{b}^{b}$ & & $\mathrm{NPC}$ & - & $\mathrm{NPC}$ \\
\hline
\end{tabular}

(b)

Figure 5: Complexity of (a) cost-optimal and (b) satisficing plan generation for fragments of UB. The "-_" mark indicates that the complexity is implied by other results in the row. All other shaded and regular cells correspond to the results obtained in this work and in the past, respectively. Empty cells correspond to open questions. Note that the only difference in our understanding of cost-optimal and satisficing planning for the fragments in question is the complexity of planning for $\mathbf{S}(1)$.

tractable and intractable problems is much more delicate. Figure 5 summarizes our current understanding of time complexity of both cost-optimal and satisficing plan generation for the $\mathbf{P}$ and $\mathbf{S}$ fragments of UB. First, in this paper we show that even the satisficing planning with directed-path singly connected, bounded in- and out-degree causal graphs is hard under 2-dependence, and that cost-optimal planning for this structural fragment of UB is hard even for 1-dependent such problems. Note that the complexity of (both cost-optimal and satisficing) plan generation for $\mathbf{P}(k)$ for $k=O(1)$ remains an interesting open problem. An additional question that remains open is the complexity of satisficing plan generation for $\mathbf{S}(1)$.

\subsection{Remarks}

Our goal in this work has been identifying new islands of tractability for cost-optimal planning, and improving by that our understanding of what makes the planning problems hard or easy to solve. Of our lesser interest was to make the poly-time algorithms practically efficient by reducing their (quite prohibitive) polynomial time complexity. In fact, in some places we intentionally sacrificed some possible optimizations to keep the already involved constructions as apprehensible as possible. Therefore, it is more than likely that the time complexity of our planning-to-COP algorithms can be further improved, or some conceptually different algorithmic ideas will be found more appropriate for the problems in question. In addition, much more efficient algorithms may work for some special cases of the general tractable families. For instance, in the paper we illustrate such possibility by presenting a low poly-time algorithm for UB problems with tree causal graphs (that is, the $\mathbf{T}$ fragment) and uniform-cost actions.

Of course, the reader may ask whether striving for practical efficiency in solving various special fragments of planning is at all motivated. As our discussion at the beginning of the paper suggests, we believe that the answer to this question is "yes". While most of the research on AI planning is rightfully devoted to solving general planning problems, many tools developed and employed in that research rely on tractable fragments of planning. For 
instance, if one works on devising an effective heuristic estimator for a planning problem by projecting it to (or embedding it in) this or another "relaxed" problem, then she will be happy to know that the latter can be solved in low poly-time. On the other hand, making a tractable fragment also efficiently solvable in practical terms is probably worth the effort only in face of some concrete "customer" of that fragment in practice.

\section{Definitions and Notation}

Starting with Definitions 1-3 from the previous section, in this section we introduce some additional definitions and notation that are used throughout the paper.

In contrast to the well-known STRIPS formalism for propositional planning, we assume that all our actions are "value changing", and this in contrast to "value setting". That is, we have $\operatorname{eff}(a)[v]$ being specified only if pre $(a)[v]$ is also specified, in which case we have $\operatorname{eff}(a)[v] \neq \operatorname{pre}(a)[v]$. While in general this assumption requires an exponential time translation, in case of unary-effect actions the translation takes only linear time. Given a UB problem $\Pi=\langle V, A, I, G\rangle$, by $A_{v} \subseteq A$ we denote the actions that change the value of $v$. Note that the unary-effectness of $\Pi$ implies that $A_{v_{1}}, \ldots, A_{v_{n}}$ is a partition of the problem actions $A$. Considering the applicability of actions, in $\mathrm{SAS}^{+}$it also helps to give a special attention and notation to the action preconditions that are left unaffected by the action. The customary name for such preconditions is prevail conditions (Bäckström \& Klein, 1991). For example, having truck $T$ and package $P$ at location $L$ are both preconditions of loading $P$ into $T$ in $L$, but only the former is a prevail condition of this action because the truck is still in $L$ after loading $P$, while $P$ is no longer there (but inside $T$ ).

Given a UB problem $\Pi=\langle V, A, I, G\rangle$, a variable subset $V^{\prime} \subseteq V$, and an arbitrary sequence of actions $\rho \in A^{*}$, by $\rho \downarrow_{V^{\prime}}$ we denote the order-preserving restriction of $\rho$ to the actions $\bigcup_{v \in V^{\prime}} A_{v}$. If the restriction is with respect to a singleton set $V^{\prime}=\{v\}$, then we allow writing $\rho \downarrow_{\{v\}}$ simply as $\rho \downarrow_{v}$. One of the key properties of cost-optimal plans for the UB problems with directed-path singly connected causal graphs is immediately derivable from Lemma 1 by Brafman and Domshlak (2003), and it is given by Corollary 1 below. Henceforth, a valid plan $\rho$ for a given problem $\Pi$ is called irreducible if any subplan $\rho^{\prime}$ of $\rho$ is not a plan for $\Pi$, in the following sense ${ }^{4}$ : Removal of any subset of (not necessarily subsequent) actions from $\rho$ makes the resulting plan either illegal, or its initial state is not $I$, or its end state is not one of the states specified by $G$.

Lemma 1 (Brafman and Domshlak, 2003) For any solvable problem $\Pi \in \mathbf{S}$ over $n$ state variables, any irreducible plan $\rho$ for $\Pi$, and any state variable $v$ in $\Pi$, the number of value changes of $v$ along $\rho$ is $\leq n$, that is, $\left|\rho \downarrow_{v}\right| \leq n$.

Corollary 1 For any solvable problem $\Pi \in \mathbf{S}$ over $n$ state variables, any cost-optimal plan $\rho$ for $\Pi$, and any state variable $v$ in $\Pi$, we have $\left|\rho \downarrow_{v}\right| \leq n$.

4. The notion of irreducible plans was introduced by Kambhampati (1995), where it was called minimal plans, and exploited for admissible pruning of partial plans during search. We adopt the terminology suggested by Brafman and Domshlak (2003) to prevent ambiguity between minimal as irreducible and minimal as optimal. 
Given an $\mathbf{S}$ problem $\Pi=\langle V, A, I, G\rangle$, we denote the initial value $I[v]$ of for each variable $v \in V$ by $\mathrm{b}_{v}$, and the opposite value by $\mathrm{w}_{v}$ (short for, black/white). Using this notation and exploiting Corollary 1, by $\sigma(v)$ we denote the longest possible sequence of values obtainable by $v$ along a cost-optimal plan $\rho$, with $|\sigma(v)|=n+1, \mathbf{b}_{v}$ occupying all the odd positions of $\sigma(v)$, and $\mathrm{w}_{v}$ occupying all the even positions of $\sigma(v)$. In addition, by $\tau(v)$ we denote a per-value time-stamping of $\sigma(v)$

$$
\tau(v)=\left\{\begin{array}{ll}
\mathrm{b}_{v}^{1} \cdot \mathrm{w}_{v}^{1} \cdot \mathrm{b}_{v}^{2} \cdot \mathrm{w}_{v}^{2} \cdots \mathrm{b}_{v}^{j+1}, & n=2 j, \\
\mathrm{~b}_{v}^{1} \cdot \mathrm{w}_{v}^{1} \cdot \mathrm{b}_{v}^{2} \cdot \mathrm{w}_{v}^{2} \cdots \mathrm{w}_{v}^{j}, & n=2 j-1,
\end{array}, j \in \mathbb{N} .\right.
$$

The sequences $\sigma(v)$ and $\tau(v)$ play an important role in our constructions both by themselves and via their prefixes and suffixes. In general, for any sequence seq, by $\unrhd[$ seq] and $\unlhd[s e q]$ we denote the set of all non-empty prefixes and suffixes of seq, respectively. In our context, a prefix $\sigma^{\prime} \in \unrhd[\sigma(v)]$ is called goal-valid if either the goal value $G[v]$ is unspecified, or the last element of $\sigma^{\prime}$ equals $G[v]$. The set of all goal-valid prefixes of $\sigma(v)$ is denoted by $\unrhd^{*}[\sigma(v)] \subseteq \unrhd[\sigma(v)]$. The notion of goal-valid prefixes is also similarly specified for $\tau(v)$.

Finally, given a $\mathrm{SAS}^{+}$problem $\Pi=\langle V, A, I, G\rangle$, a subset of state variables $V^{\prime} \subseteq V$, and an action sequence $\rho \in A^{*}$, we say that $\rho$ is applicable with respect to $V^{\prime}$ if restricting the preconditions and effects of the actions in $\rho$ to the variables $V^{\prime}$ makes $\rho$ applicable in $I$.

\section{Cost-Optimal Planning for $\mathbf{P}_{b}$}

This section is devoted to the proof of tractability of cost-optimal planning for the problem fragment $\mathbf{P}_{b}$. We begin with describing our planning-to-COP scheme for $\mathbf{P}_{b}$, and then prove its correctness and complexity. Finally, we present a interesting subset of $\mathbf{P}_{b}$ for which costoptimal planning is not only tractable, but also provably solvable in low polynomial time.

\subsection{Construction}

Before we proceed with the details of the construction, we make an assumption that our actions are fully specified in terms of the variables' parents in the causal graph. If $\operatorname{pred}(v) \subset$ $V$ denotes the set of all the immediate predecessors of $v$ in the causal graph $C G(\Pi)$, then we assume that, for each action $a \in A_{v}$, pre $(a)[w]$ is specified for each $w \in \operatorname{pred}(v)$. While in general such an assumption requires an exponential translation, this is not the case with $\mathbf{P}_{b}$. Let $A^{\bowtie}$ be such a translation of the original problem actions $A$. To obtain $A^{\bowtie}$, for every variable $v \in V$, every action in $A_{v}$ is represented in $A^{\bowtie}$ by a set of actions that are preconditioned by complete assignments to $\operatorname{pred}(v)$. If $|\operatorname{pred}(v)|=k$, and the precondition of $a$ is specified only in terms of some $0 \leq k^{\prime} \leq k$ parents of $v$, then $a$ is represented in $A^{\bowtie}$ by a set of actions, each extending the precondition pre $(a)$ by a certain instantiation of the previously unspecified $k-k^{\prime}$ parents of $v$, and having the cost $\mathcal{C}\left(a^{\prime}\right)=\mathcal{C}(a)$. Note that the expansions of two or more original actions may overlap, and thus $A^{\bowtie}$ may contain syntactically identical yet differently priced actions. Without loss of generality, we assume that only a minimally-priced such clone is kept in $A^{\bowtie}$. The key point is that compiling $A$ into $A^{\bowtie}$ for the $\mathbf{P}_{b}$ problems is poly-time, as the procedure is linear in $\left|A^{\bowtie}\right|=O\left(n 2^{\ln (\Pi)+1}\right)$. Finally, the (straightforward to prove) Proposition 1 summarizes the correctness of our assumption with respect to the cost-optimal planning for UB. 
Proposition 1 For any UB problem $\Pi=\langle V, A, I, G\rangle$, the cost of the optimal plans for $\Pi$ is equal to this for $\Pi^{\bowtie}=\left\langle V, A^{\bowtie}, I, G\right\rangle$, with optimal plans for $\Pi$ being reconstructible in linear time from the optimal plans for $\Pi^{\bowtie}$ and vice versa.

We now specify our compilation of a given $\mathbf{P}_{b}$ problem $\Pi$ into a constraint optimization problem $\mathrm{COP}_{\Pi}$. The $\mathrm{COP}$ variable set $\mathcal{X}$ contains a variable $x_{v}$ for each planning variable $v \in V$, and the domain $\operatorname{Dom}\left(x_{v}\right)$ consists of all valid prefixes of $\tau(v)$. That is,

$$
\begin{aligned}
\mathcal{X} & =\left\{x_{v} \mid v \in V\right\} \\
\operatorname{Dom}\left(x_{v}\right) & =\unrhd^{*}[\tau(v)]
\end{aligned}
$$

Informally, the domain of each variable $x_{v}$ contains all possible sequences of values that the planning variable $v$ may undergo along a cost-optimal plan. Now, for each planning variable $v$ with parents $\operatorname{pred}(v)=\left\{w_{1}, \ldots, w_{k}\right\}$, the set of COP functions $\mathcal{F}$ contains a single non-negative, real-valued function $\varphi_{v}$ with the scope

$$
Q_{v}=\left\{x_{v}, x_{w_{1}}, \ldots, x_{w_{k}}\right\}
$$

The purpose of these functions is to connect the value-changing sequences of $v$ and these of its parents $\operatorname{pred}(v)$. The specification of these functions is the more involved part of the compilation.

First, for each planning variable $v$ with $\operatorname{pred}(v)=\emptyset$, and each of its goal-valid (timestamped) value-changing sequences $\tau^{\prime} \in \unrhd^{*}[\tau(v)]$, we set

$$
\varphi_{v}\left(\tau^{\prime}\right)= \begin{cases}0, & \left|\tau^{\prime}\right|=1 \\ \mathfrak{C}\left(a_{\mathrm{w}_{v}}\right), & \left|\tau^{\prime}\right|=2 \\ \left\lfloor\frac{\left|\tau^{\prime}\right|}{2}\right\rfloor \cdot \mathfrak{C}\left(a_{\mathrm{w}_{v}}\right)+\left\lfloor\frac{\left|\tau^{\prime}\right|-1}{2}\right\rfloor \cdot \mathfrak{C}\left(a_{\mathbf{b}_{v}}\right), & \text { otherwise }\end{cases}
$$

where $\operatorname{eff}\left(a_{\mathrm{w}_{v}}\right)[v]=\left\{\mathrm{w}_{v}\right\}, \operatorname{eff}\left(a_{\mathrm{b}_{v}}\right)[v]=\left\{\mathrm{b}_{v}\right\}$, and $\mathfrak{C}(a)=\mathcal{C}(a)$ if $a \in A$, and $\infty$, otherwise. It is not hard to verify that $\varphi_{v}\left(\tau^{\prime}\right)$ corresponds to the optimal cost of performing $\left|\sigma^{\prime}\right|-1$ value changes of $v$ in $\Pi$.

Now, for each non-root variable $v$ with $\operatorname{pred}(v)=\left\{w_{1}, \ldots, w_{k}\right\}, k \geq 1$, we specify the function $\varphi_{v}$ as follows. For each goal-valid value-changing sequence $\tau^{\prime} \in \unrhd^{*}[\tau(v)]$ of $v$, and each set of such goal-valid value-changing sequences $\left\{\tau_{1}^{\prime} \in \unrhd^{*}\left[\tau\left(w_{1}\right)\right], \ldots, \tau_{k}^{\prime} \in \unrhd^{*}\left[\tau\left(w_{k}\right)\right]\right\}$ of $v$ 's parents, we want to set $\varphi_{v}\left(\tau^{\prime}, \tau_{1}^{\prime}, \ldots, \tau_{k}^{\prime}\right)$ to the optimal cost of performing $\left|\tau^{\prime}\right|-1$ value changes of $v$, given that $w_{1}, \ldots, w_{k}$ change their values $\left|\tau_{1}^{\prime}\right|-1, \ldots,\left|\tau_{k}^{\prime}\right|-1$ times, respectively. In what follows, we reduce setting the value $\varphi_{v}\left(\tau^{\prime}, \tau_{1}^{\prime}, \ldots, \tau_{k}^{\prime}\right)$ to solving a single-source shortest path problem on an edge-weighted digraph $G_{e}^{\prime}(v)$ that slightly enhances a similarly-named graphical structure suggested by Brafman and Domshlak (2003). Despite the substantial similarity, we provide our construction of $G_{e}^{\prime}(v)$ in full details to save the reader patching the essential differences.

Given the value-changing sequences $\tau_{1}^{\prime}, \ldots, \tau_{k}^{\prime}$ as above, the digraph $G_{e}^{\prime}(v)$ is created in three steps. First, we construct a labeled directed graph $G(v)$ capturing information about all sequences of assignments on $\operatorname{pred}(v)$ that can enable $n$ or less value flips of $v$. The graph $G(v)$ is defined as follows:

1. $G(v)$ consist of $\eta=\max _{\tau^{\prime} \in \unrhd^{*}[\tau(v)]}\left|\tau^{\prime}\right|$ nodes. 
2. $G(v)$ forms a 2-colored multichain, i.e., (i) the nodes of the graph are colored with black (b) and white (w), starting with black; (ii) there are no two subsequent nodes with the same color; (iii) for $1 \leq i \leq \eta-1$, edges from the node $i$ are only to the node $i+1$.

Observe that such a construction of $G(v)$ promises that the color of the last node will be consistent with the goal value $G[v]$ if such is specified.

3. The nodes of $G(v)$ are denoted precisely by the elements of the longest goal-valid value-changing sequence $\tau^{\prime} \in \unrhd^{*}[\tau(v)]$, that is, $\mathrm{b}_{v}^{i}$ stands for the $i$ th black node in $G(v)$.

4. Suppose that there are $m$ operators in $A_{v}$ that, under different preconditions, change the value of $v$ from $\mathbf{b}_{v}$ to $\mathbf{w}_{v}$. In this case, for each $i$, there are $m$ edges from $\mathbf{b}_{v}^{i}$ to $\mathbf{w}_{v}^{i}$, and $\left|A_{v}\right|-m$ edges from $\mathbf{w}_{v}^{i}$ to $\mathbf{b}_{v}^{i+1}$. Each such edge $e$ is labeled with the cost of the corresponding action, as well as with the prevail conditions of that action, which is a $k$-tuple of the values of $w_{1}, \ldots, w_{k}$. This compound label of $e$ is denoted by $l(e)$, and the prevail condition and cost parts of $l(e)$ are henceforth denoted by $\operatorname{prv}(e)$ and $\mathcal{C}(e)$, respectively.

As the formal definition of $G(v)$ is somewhat complicated, we provide an illustrating example. Suppose that we are given a $\mathbf{P}_{b}$ problem over 5 variables, and we consider a variable $v$ with $\operatorname{pred}(v)=\{u, w\}, I[v]=\mathrm{b}_{v}$, and $G[v]=\mathrm{w}_{v}$. Let

$$
A_{v}=\left\{\begin{array}{l}
a_{1}: \operatorname{pre}\left(a_{1}\right)=\left\{\mathrm{b}_{v}, \mathrm{~b}_{u}, \mathrm{w}_{w}\right\}, \operatorname{eff}\left(a_{1}\right)=\left\{\mathrm{w}_{v}\right\}, \mathcal{C}\left(a_{1}\right)=\alpha_{1} \\
a_{2}: \operatorname{pre}\left(a_{2}\right)=\left\{\mathrm{w}_{v}, \mathrm{~b}_{u}, \mathrm{~b}_{w}\right\}, \operatorname{eff}\left(a_{2}\right)=\left\{\mathrm{b}_{v}\right\}, \mathcal{C}\left(a_{2}\right)=\alpha_{2} \\
a_{3}: \operatorname{pre}\left(a_{3}\right)=\left\{\mathrm{w}_{v}, \mathrm{w}_{u}, \mathrm{w}_{w}\right\}, \operatorname{eff}\left(a_{3}\right)=\left\{\mathrm{b}_{v}\right\}, \mathcal{C}\left(a_{3}\right)=\alpha_{3}
\end{array}\right.
$$

The corresponding graph $G(v)$ is depicted in Figure 6a. Informally, the graph $G(v)$ captures information about all potential executions of the actions in $A_{v}$ along a cost-optimal plan for $\Pi$. Each path from the source node of $G(v)$ uniquely corresponds to one such execution. Although the number of these alternative executions may be exponential in $n$, their graphical representation via $G(v)$ is compact-the number of edges in $G(v)$ is $O\left(n \cdot\left|A_{v}\right|\right)$. Note that the information about the number of times each action in $A_{v}$ can be executed is not captured by $G(v)$. The following two steps add this essential information into the graphical structure.

At the second step, the digraph $G(v)=(V, E)$ is expanded into a digraph $G^{\prime}(v)=$ $\left(V^{\prime}, E^{\prime}\right)$ by substituting each edge $e \in E$ with a set of edges (between the same nodes), but with the labels corresponding to all possible assignments of the elements of $\tau_{1}^{\prime}, \ldots, \tau_{k}^{\prime}$ to $\operatorname{prv}(e)$. For example, an edge $e \in E$ labeled with $\left\|\mathrm{b}_{w_{1}} \mathrm{~b}_{w_{2}}, 10\right\|$ might be substituted in $E^{\prime}$ with edges labeled with $\left\{\left\|\mathrm{b}_{w_{1}}^{1} \mathrm{~b}_{w_{2}}^{1}, 10\right\|,\left\|\mathrm{b}_{w_{1}}^{1} \mathrm{~b}_{w_{2}}^{2}, 10\right\|,\left\|\mathrm{b}_{w_{1}}^{2} \mathrm{~b}_{w_{2}}^{1}, 10\right\|, \ldots\right\}$. Finally, we set $V^{\prime}=V \cup\left\{s_{v}, t_{v}\right\}$, and add a single edge labeled with the first elements of $\tau_{1}^{\prime}, \ldots, \tau_{k}^{\prime}$ and zero cost (that is, $\left.\left\|\mathrm{b}_{w_{1}}^{1} \cdots \mathrm{b}_{w_{k}}^{1}, 0\right\|\right)$ from $s_{v}$ to the original source node $\mathrm{b}_{v}^{1}$, plus a single edge labeled with the last elements of $\tau_{1}^{\prime}, \ldots, \tau_{k}^{\prime}$ and zero cost from the original sink node of $G(v)$ to $t_{v}$. Informally, the digraph $G^{\prime}(v)$ can be viewed as a projection of the value-changing sequences $\tau_{1}^{\prime}, \ldots, \tau_{k}^{\prime}$ on the base digraph $G(v)$. Figure $6 \mathrm{~b}$ illustrates $G^{\prime}(v)$ for the example above, assuming $\tau_{u}^{\prime}=\mathrm{b}_{u}^{1} \cdot \mathrm{w}_{u}^{1} \cdot \mathrm{b}_{u}^{2} \cdot \mathrm{w}_{u}^{2} \cdot \mathrm{b}_{u}^{3}$ and $\tau_{w}=\mathrm{b}_{w}^{1} \cdot \mathrm{w}_{w}^{1} \cdot \mathrm{b}_{w}^{2} \cdot \mathrm{w}_{w}^{2}$.

At the third step, a digraph $G_{e}^{\prime}(v)=\left(V_{e}^{\prime}, E_{e}^{\prime}\right)$ is constructed from $G^{\prime}(v)$ as follows. 


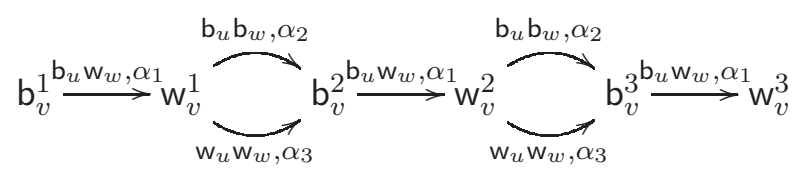

(a)

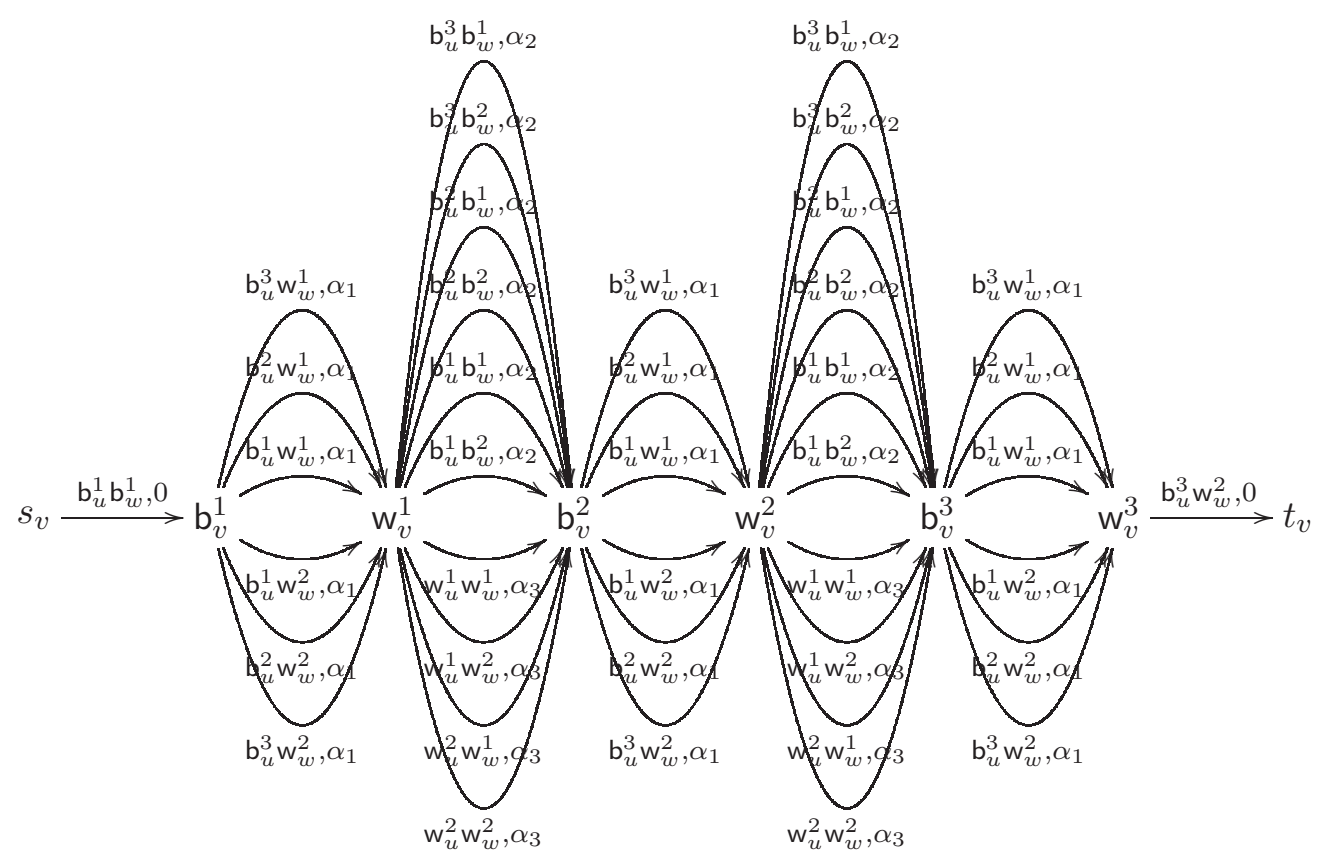

(b)

Figure 6: Example of the graphs (a) $G(v)$, and (b) $G^{\prime}(v)$.

(i) The nodes $V_{e}^{\prime}$ correspond to the edges of $G^{\prime}(v)$.

(ii) The edges $\left(v_{e}, v_{e^{\prime}}\right) \in E_{e}^{\prime}$ correspond to all pairs of immediately consecutive edges $e, e^{\prime} \in E^{\prime}$ such that, for $1 \leq i \leq k$, either $\operatorname{prv}(e)\left[w_{i}\right]=\operatorname{prv}\left(e^{\prime}\right)\left[w_{i}\right]$, or $\operatorname{prv}\left(e^{\prime}\right)\left[w_{i}\right]$ appears after $\operatorname{prv}(e)\left[w_{i}\right]$ along $\tau_{i}^{\prime}$.

(iii) Each edge $\left(v_{e}, v_{e^{\prime}}\right) \in E_{e}^{\prime}$ is weighted with $\mathcal{C}\left(e^{\prime}\right)$.

Figure 7 depicts the graph $G_{e}^{\prime}(v)$ for our example.

Assuming $\alpha_{3} \leq \alpha_{2}$, the dashed edges correspond to the minimal-cost path of length 5 from the dummy source node $\mathbf{b}_{u}^{1} \mathrm{~b}_{w}^{1}$. Note that, if the costs of actions $A_{v}$ is all we care about, this path corresponds to the cost-optimal sequence of 5 value changes of $v$ starting from its initial value $\mathrm{b}_{v}$ in $\Pi$. In fact, not only this path corresponds to such a cost-optimal sequence, but it also explicitly describes the underlying sequence of actions from $A_{v}$, as well as the total cost of these actions. Finally, for all $0 \leq i \leq n$, such minimal-cost paths of length $i$ can be determined by running on $G_{e}^{\prime}(v)$ a low-polynomial single-source shortest 


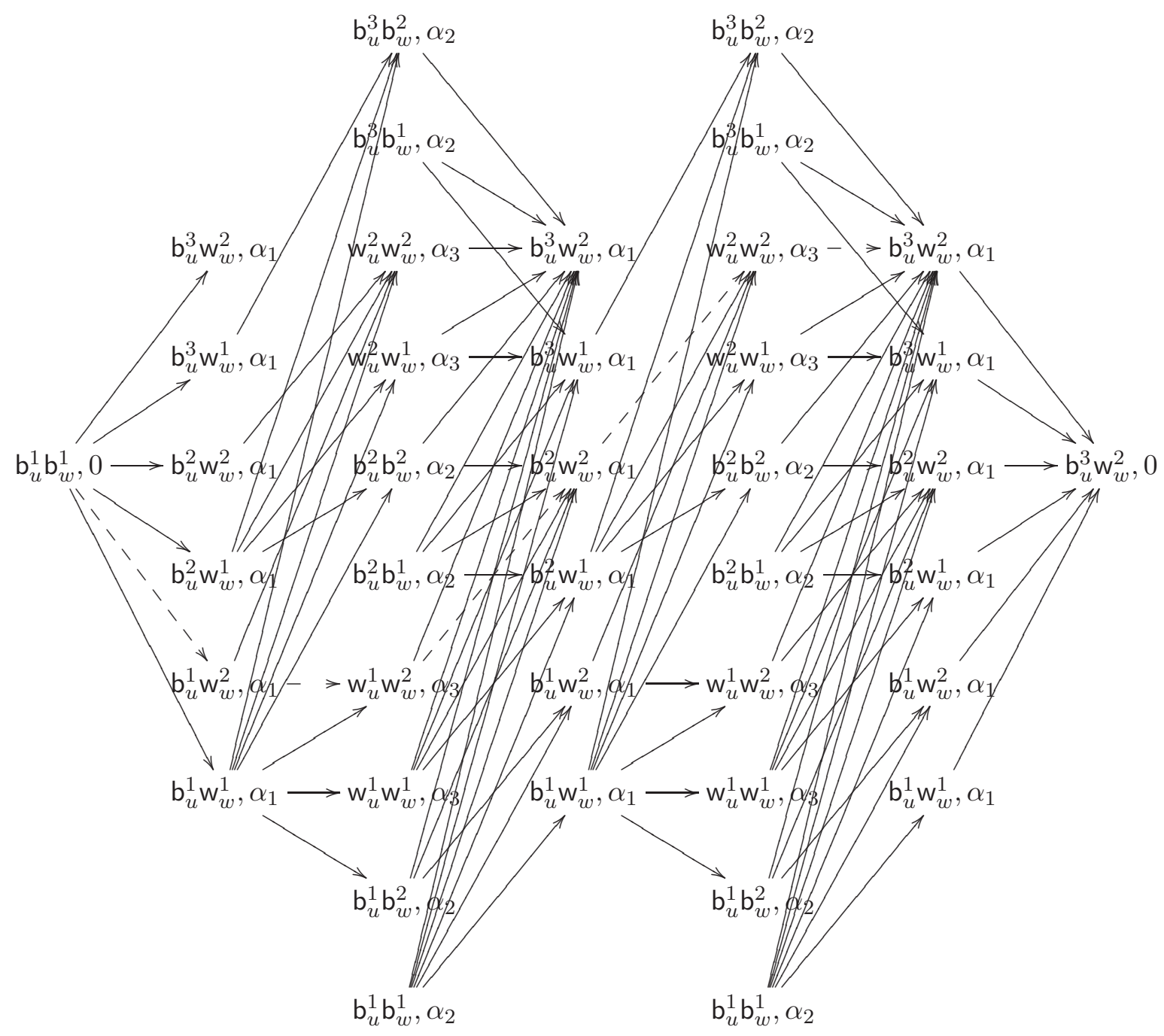

Figure 7: The graph $G_{e}^{\prime}(v)$ constructed from the graph $G^{\prime}(v)$ in Fugure 6b.

paths algorithm by Dijkstra (Cormen, Leiserson, \& Rivest, 1990). This property of the graph $G_{e}^{\prime}(v)$ provides us with the last building block for our algorithm for cost-optimal planning for $\mathbf{P}_{b}$.

The overall algorithm for cost-optimal planning for $\mathbf{P}_{b}$ that is based on the above construction is depicted in Figure 8. Given a problem $\Pi \in \mathbf{P}_{b}$, the algorithm compiles it into the constraint optimization problem $\mathrm{COP}_{\Pi}$, and solves it using a standard algorithm for constraint optimization over tree constraint networks (Dechter, 2003). The specification of $\mathrm{COP}_{\Pi}$ has already been explained inline. We believe it is already intuitive that this compilation takes time polynomial in the description size of $\Pi$, but in the next section we also prove it formally. Solving $\mathrm{COP}_{\Pi}$ using the algorithm for tree-structured constraint networks can be done in time polynomial in the description size of $\mathrm{COP}_{\Pi}$ because 


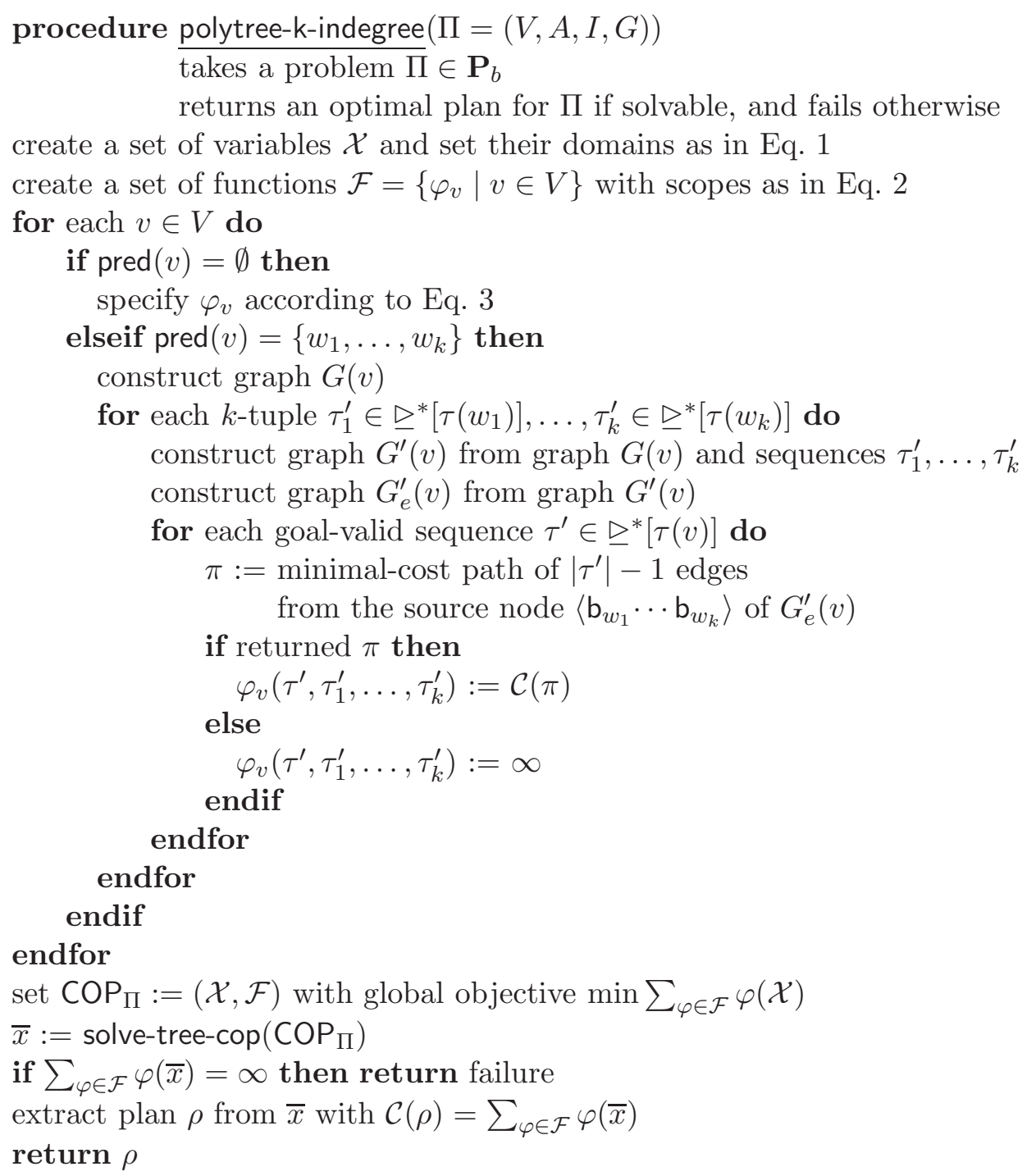

Figure 8: Algorithm for cost-optimal planning for $\mathbf{P}_{b}$.

(i) the tree-width of the cost network of $\mathrm{COP}_{\Pi}$ is bounded by the same constant that bounds the in-degree of the causal graph, and

(ii) optimal tree-decomposition of the $\mathrm{COP}_{\Pi}$ 's cost network is given by any topological ordering of the causal graph.

\subsection{Correctness and Complexity}

We now proceed with proving both the correctness and the polynomial time complexity of our algorithm for $\mathbf{P}_{b}$. We begin with proving in Theorem 1 below a rather general property of polytrees that helps us analyzing both the $\mathbf{P}_{b}$ fragment in question, as well as the $\mathbf{P}(1)$ fragment considered later in the paper. We note that a special case of this property has 
been already exploited in the past in the proof of Lemma 2 by Brafman and Domshlak (2003), but, to our knowledge, this property has never been formulated as a generic claim of Theorem 1. Throughout the paper we then demonstrate that this generic claim can be helpful in numerous situations; the proof of Theorem 1 is in Appendix A, p. 245.

Theorem 1 Let $G$ be a polytree over vertices $V=\{1, \ldots, n\}$, and $\operatorname{pred}(i) \subset V$ denote the immediate predecessors of $i$ in $G$. For each $i \in V$, let $O_{i}$ be a finite set of objects associated with the vertex $i$, with the sets $O_{1}, \ldots, O_{n}$ being pairwise disjoint. For each $i \in V$, let $>_{i}$ be a strict partial order over $O_{i}$, and, for each $j \in \operatorname{pred}(i)$, let $>_{i, j}$ be a strict partial order over $O_{i} \cup O_{j}$.

If, for each $i \in V, j \in \operatorname{pred}(i)$, the transitively closed $>_{i} \cup>_{i, j}$ and $>_{j} \cup>_{i, j}$ induce (strict) partial orders over $O_{i} \cup O_{j}$, then so does the transitively closed

$$
>=\bigcup_{i \in V}\left(>_{i} \cup \bigcup_{j \in \operatorname{pred}(i)}>_{i, j}\right)
$$

over $O=\bigcup_{i \in V} O_{i}$.

Using Theorem 1 we now proceed with proving the correctness and complexity of the polytree-k-indegree algorithm.

Theorem 2 Let $\Pi$ be a planning problem in $\mathbf{P}_{b}, \operatorname{COP}_{\Pi}=(\mathcal{X}, \mathcal{F})$ be the corresponding constraint optimization problem, and $\bar{x} \in \operatorname{Dom}(\mathcal{X})$ be an optimal solution for $\mathrm{COP}_{\Pi}$ with $\sum_{\varphi \in \mathcal{F}} \varphi(\bar{x})=\alpha$.

(I) If $\alpha<\infty$, then a plan of cost $\alpha$ for $\Pi$ can be reconstructed from $\bar{x}$ in time polynomial in the description size of $\Pi$.

(II) If $\Pi$ has a plan, then $\alpha<\infty$.

Proof Sketch: The proof of Theorem 2 is in Appendix A. p. 247. To prove (I), given a COP solution $\bar{x}=\left\{\tau_{v_{1}}, \ldots, \tau_{v_{n}}\right\}$ with $\sum_{\varphi \in \mathcal{F}} \varphi(\bar{x})=\alpha<\infty$, we construct a plan $\rho$ for $\Pi$ with $\mathcal{C}(\rho)=\alpha$. This is done by constructing action sequences $\rho_{v}$ for each $v \in V$, as well as constructing partial orders over the elements of these sequences of each variable and its parents. These orders are then combined and linearized (using Theorem 1) into an action sequence $\rho$ that is a valid plan for $\Pi$ with $\mathcal{C}(\rho)=\sum_{v \in V} \mathcal{C}\left(\rho_{v}\right)=\sum_{v \in V} \varphi_{v}(\bar{x})=$ $\sum_{\varphi \in \mathcal{F}} \varphi(\bar{x})=\alpha$. To prove (II), given a solvable problem $\Pi$ and some irreducible plan $\rho$, we construct a COP assignment $\bar{x}_{\rho}$ such that $\sum_{\varphi \in \mathcal{F}} \varphi\left(\bar{x}_{\rho}\right)=\mathcal{C}(\rho)$. Then, from $\alpha \leq \sum_{\varphi \in \mathcal{F}} \varphi\left(\bar{x}_{\rho}\right)$ and $\mathcal{C}(\rho)<\infty$, we obtain the claimed $\alpha<\infty$.

Theorem 3 Cost-optimal planning for $\mathbf{P}_{b}$ is tractable.

Proof: Given a planning problem $\Pi \in \mathbf{P}_{b}$, we show that the corresponding constraint optimization problem $\mathrm{COP}_{\Pi}$ can be constructed and solved in time polynomial in the description size of $\Pi$.

Let $n$ be the number of state variables in $\Pi$, and $\kappa$ be the maximal node in-degree in the causal graph $C G(\Pi)$. In polytree-k-indegree, for each planning variable $v \in V$ with $\operatorname{pred}(v)=\left\{w_{1}, \ldots, w_{k}\right\}$, and each $k$-tuple $\tau_{1}^{\prime} \in \unrhd^{*}\left[\tau\left(w_{1}\right)\right], \ldots, \tau_{k}^{\prime} \in \unrhd^{*}\left[\tau\left(w_{k}\right)\right]$, we 
(i) construct the graph $G_{e}^{\prime}(v)$, and

(ii) use the Dijkstra algorithm to compute shortest paths from the source node of $G_{e}^{\prime}(v)$ to all other nodes in that graph.

For each $w_{i}$, we have $\tau\left(w_{i}\right)=n$, and thus the number of $k$-tuples as above for each $v \in V$ is $O\left(n^{k}\right)$. For each such $k$-tuple, the corresponding graph $G_{e}^{\prime}(v)$ can be constructed in time linear in the number of its edges $=O\left(n^{2 k+2} \cdot\left|A_{v}\right|^{2}\right)=O\left(n^{2 k+2} \cdot 2^{2 k+2}\right)($ Brafman \& Domshlak, 2003). The time complexity of the Dijkstra algorithm on a digraph $G=(N, E)$ is $O(E \log (N))$, and on $G_{e}^{\prime}(v)$ it gives us $O\left(n^{2 k+2} \cdot 2^{2 k+2} \cdot \log \left(n^{k+1} \cdot 2^{k+1}\right)\right)$. Putting things together, the complexity of constructing $\mathrm{COP}_{\Pi}$ is

$$
O\left(n^{3 \kappa+3} \cdot 2^{2 \kappa+2} \cdot \log \left(n^{\kappa+1} \cdot 2^{\kappa+1}\right)\right) .
$$

Applying a tree-decomposition of $\mathrm{COP}_{\Pi}$ along the scopes of its functional components we arrive into an equivalent, tree-structured constraint optimization problem over $n$ variables with domains of size $O\left(n^{\kappa+1}\right)$. This COP is defined by the hard binary "compatibility" constraints between the variables, and costs associated with the variables' values. Such a tree-structured COP can be solved in time $O\left(x y^{2}\right)$ where $x$ is the number of variables and $y$ is an upper bound on the size of a variable's domain (Dechter, 2003). Therefore, solving our $\mathrm{COP}_{\Pi}$ can be done in time $O\left(n^{2 \kappa+3}\right)$. As the expression in Eq. 4 dominates both $O\left(n^{2 \kappa+3}\right)$, and the time complexity of extracting a plan from the optimal solution to $\mathrm{COP}_{\Pi}$ (see the proof of (I) in Theorem 2), the overall complexity of the algorithm polytree-k-indegree is given by Eq. 4 . And since in $\mathbf{P}_{b}$ we have $\kappa=O(1)$, we conclude that the complexity of polytree-k-indegree is polynomial in the description size of $\Pi$.

\subsection{Towards Practically Efficient Special Cases}

The polytree-k-indegree algorithm for $\mathbf{P}_{b}$ is polynomial, but is rather involved and its complexity is exponential in $\ln (C G(\Pi))$. It is quite possible that more efficient algorithms for $\mathbf{P}_{b}$, or some of its fragments can be devised. Indeed, below we show that a simple algorithm for $\mathbf{T} \subset \mathbf{P}_{b}$ problems has already appeared in the literature in a different context, but it was never checked to when (if at all) it provides cost-optimal solutions. This is the TreeDT algorithm for preferential reasoning with tree-structured CP-nets (Boutilier, Brafman, Domshlak, Hoos, \& Poole, 2004), and it turns out that its straightforward adaptation for $\mathbf{T}$ planning problems always provides cost-optimal solutions for $\mathbf{T}$ problems with uniform-cost actions. The algorithm is depicted in Figure 9, and it is not hard to verify that its time complexity is linear in the length of the generated plan $\rho$-all it does is iteratively "removing" the parts of the problem that can be safely ignored in the later steps, and then applying a value-changing action on a lowest (in the causal graph) variable for which such an action exists.

Theorem 4 Given a $\mathbf{T}$ problem $\Pi$ with uniform-cost actions over $n$ state variables,

(I) the algorithm tree-uniform-cost finds a plan if and only if $\Pi$ is solvable,

(II) if the algorithm tree-uniform-cost finds a plan for $\Pi$, then this plan is cost-optimal, and 


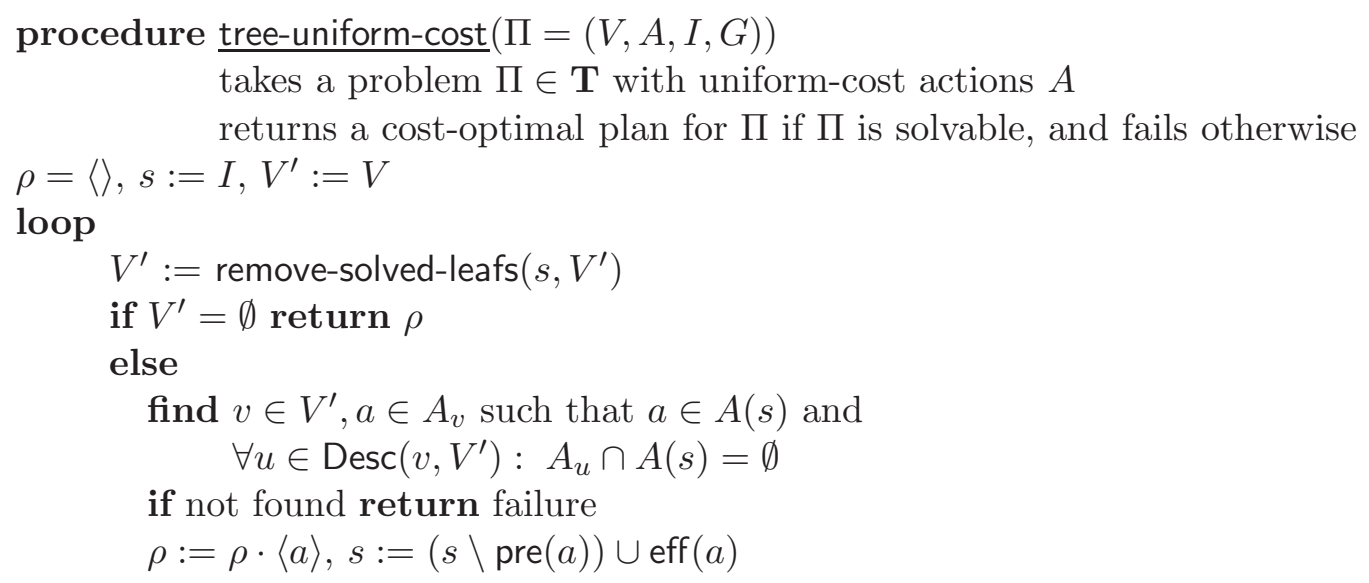

Figure 9: A simple algorithm for cost-optimal planning for $\mathbf{T}$ problems with uniform-cost actions. The notation $\operatorname{Desc}\left(v, V^{\prime}\right)$ stands for the subset of $V^{\prime}$ containing the descendants of $v$ in $C G(\Pi)$, and $A(s)$ stands for the set of all actions applicable in the state $s$.

(III) the time complexity of tree-uniform-cost is $\Theta\left(n^{2}\right)$.

Proof: Without loss of generality, in what follows we assume that the actions of $\Pi$ are all unit-cost, that is, for each plan $\rho$ for $\Pi, \mathcal{C}(\rho)=|\rho|$.

(I) Straightforward by reusing as is the proof of Theorem 11 by Boutilier et al. (2004).

(II) Assume to the contrary that the plan $\rho$ provided by tree-uniform-cost is not optimal, that is, there exists a plan $\rho^{\prime}$ such that $\left|\rho^{\prime}\right|<|\rho|$. In particular, this implies existence of a variable $v$ such that $\left|\rho^{\prime} \downarrow_{v}\right|<\left|\rho \downarrow_{v}\right|$. The semantics of planning implies that

$$
\left|\rho^{\prime} \downarrow_{v}\right| \leq\left|\rho \downarrow_{v}\right|-\left(\epsilon_{v}+1\right)
$$

where $\epsilon_{v}=1$ if $G[v]$ is specified, and 0 otherwise. Likewise, since the causal graph $C G(\Pi)$ forms a directed tree, there exists a variable $v$ satisfying Eq. 5 such that, for all the descendants $u$ of $v$ in $C G(\Pi)$ holds:

$$
\left|\rho^{\prime} \downarrow_{u}\right| \geq\left|\rho \downarrow_{u}\right|
$$

Let $C h(v)$ be the set of all the immediate descendants of $v$ in $C G(\Pi)$. By the construction of tree-uniform-cost, we have that:

1. If $C h(v)=\emptyset$, then $\left|\rho \downarrow_{v}\right| \leq \epsilon_{v}$, and this contradicts Eq. 5 as $\left|\rho^{\prime} \downarrow_{v}\right|$ is a non-negative quantity by definition.

2. Otherwise, if $C h(v) \neq \emptyset$, then, by the construction of tree-uniform-cost, there exists $u \in C h(v)$ such that changing its value $\left|\rho \downarrow_{u}\right|$ times requires changing the value of $v$ at least $\left|\rho \downarrow_{v}\right|-\epsilon_{v}$ times. In other words, there is no action sequence $\varrho$ applicable 
in $I$ such that $\left|\varrho \downarrow_{u}\right| \geq\left|\rho \downarrow_{u}\right|$ while $\left|\varrho \downarrow_{v}\right|<\left|\rho \downarrow_{v}\right|-\epsilon_{v}$. However, from Eq. 6 we have $\left|\rho^{\prime} \downarrow_{u}\right| \geq\left|\rho \downarrow_{u}\right|$, and thus $\left|\rho^{\prime} \downarrow_{v}\right|$ has to be at least $\left|\rho \downarrow_{v}\right|-\epsilon_{v}$. This, however, contradicts Eq. 5.

Hence, we have proved that $\left|\rho^{\prime} \downarrow_{v}\right| \geq\left|\rho \downarrow_{v}\right|$, contradicting our assumption that $\left|\rho^{\prime}\right|<|\rho|$.

(III) Implied by Theorems 12 and 13 by Boutilier et al. (2004).

The requirement in Theorem 4 for all actions to have the same cost is essential. The example below shows that in more general case the algorithm tree-uniform-cost is no longer cost-optimal. Consider $\Pi=(V, A, I, G) \in \mathbf{T}$ with $V=\{v, u\}, I=\left\{\mathrm{b}_{v}, \mathrm{~b}_{u}\right\}, G=\left\{\mathrm{b}_{v}, \mathrm{w}_{u}\right\}$, and $A=\left\{a_{1}, a_{2}, a_{3}, a_{4}\right\}$ with

$$
\begin{aligned}
\operatorname{eff}\left(a_{1}\right) & =\left\{\mathrm{w}_{v}\right\}, \operatorname{pre}\left(a_{1}\right)=\left\{\mathrm{b}_{v}\right\} \\
\operatorname{eff}\left(a_{2}\right) & =\left\{\mathrm{b}_{v}\right\}, \operatorname{pre}\left(a_{2}\right)=\left\{\mathrm{w}_{v}\right\} \\
\operatorname{eff}\left(a_{3}\right) & =\left\{\mathrm{w}_{u}\right\}, \operatorname{pre}\left(a_{3}\right)=\left\{\mathrm{b}_{u}, \mathrm{w}_{v}\right\} \\
\operatorname{eff}\left(a_{4}\right)= & \left\{\mathrm{w}_{u}\right\}, \operatorname{pre}\left(a_{4}\right)=\left\{\mathrm{b}_{u}, \mathrm{~b}_{v}\right\} \\
\mathcal{C}\left(a_{1}\right) & =\mathcal{C}\left(a_{2}\right)=\mathcal{C}\left(a_{3}\right)=1 \\
\mathcal{C}\left(a_{4}\right) & =4
\end{aligned}
$$

On this problem, the tree-uniform-cost algorithm returns $\rho=\left\langle a_{4}\right\rangle$ with $\mathcal{C}(\rho)=4$, while the optimal plan is $\rho^{\prime}=\left\langle a_{1}, a_{3}, a_{2}\right\rangle$ with $\mathcal{C}\left(\rho^{\prime}\right)=3$.

\section{Cost-Optimal Planning for $\mathrm{P}(1)$ with Uniform-Cost Actions}

In this section we provide a polynomial time algorithm for cost-optimal planning for $\mathbf{P}(1)$ problems with uniform-cost actions. We begin with showing that such problems exhibit an interesting property, then we exploit this property for devising a planning-to-COP scheme for these problems, and then prove the correctness and complexity of the algorithm.

We begin with providing some useful notation. Given a $\mathbf{P}(1)$ problem $\Pi=(V, A, I, G)$, for each $v \in V$, each $w \in \operatorname{pred}(v)$, and each $\alpha \in\left\{\mathrm{b}_{v}, \mathrm{w}_{v}\right\}, \beta \in\left\{\mathrm{b}_{w}, \mathrm{w}_{w}\right\}$, by $a_{\alpha \mid \beta}$ we denote the action $a$ with $\operatorname{eff}(a)[v]=\alpha$ and $\operatorname{pre}(a)[w]=\beta$. Since $\Pi$ is 1-dependent, the applicability of $a_{\alpha \mid \beta}$ is prevailed only by the value of $w$. It is important to keep in mind that $a_{\alpha \mid \beta}$ is just a notation; the action $a_{\alpha \mid \beta}$ may not belong to the action set $A$ of $\Pi$.

\subsection{Post-Unique Plans and P(1) Problems}

We now proceed with introducing the notion of post-unique action sequences that plays a key role in our planning-to-COP compilation here.

Definition 4 Let $\Pi=(V, A, I, G)$ be a UB problem instance. An action sequence $\varrho \in A^{*}$ is called post-unique if, for each pair of actions a, $a^{\prime} \in \varrho$, we have $\operatorname{eff}(a)=\operatorname{eff}\left(a^{\prime}\right)$ only if $a=a^{\prime}$. That is, all the changes of each variable to a certain value along @ are performed by the same (type of) action. The (possibly empty) set of all post-unique plans for $\Pi i$ is denoted by $\mathcal{P}^{p u}(\Pi)$ (or simply $\mathcal{P}^{p u}$, if the identity of $\Pi$ is clear from the context). 
The notion of post-unique action sequences is closely related to the notion of postunique planning problems (Bäckström \& Klein, 1991; Bäckström \& Nebel, 1995), but is considerably weaker than the latter. While action sets of post-unique planning problems are not allowed to contain two actions with the same effect, Definition 4 poses a similar restriction only on action sequences, and not on the underlying planning problems. Still, the property of post-uniqueness for plans is strong. In general, solvable problems in UB may not exhibit post-unique plans at all. Turns out, however, that for the problems in $\mathbf{P}(1)$ this is very much not the case.

Theorem 5 For any solvable $\mathbf{P}(1)$ problem $\Pi=(V, A, I, G)$, we have $\mathcal{P}^{p u}(\Pi) \neq \emptyset$. Moreover, if the actions $A$ are uniform-cost, then $\mathcal{P}^{p u}(\Pi)$ contains at least one cost-optimal plan.

Proof: As the correctness of the second claim immediately implies the correctness of the first one, we focus on the proof the second claim. Given a $\mathbf{P}(1)$ problem $\Pi=(V, A, I, G)$ with uniform-cost actions, and plan $\rho=\left\langle a_{1}, \ldots, a_{m}\right\rangle$ for $\Pi$, we construct a sequence of actions $\rho^{*}$ such that:

- $\rho^{*}$ is a post-unique plan for $\Pi$,

- $\mathcal{C}\left(\rho^{*}\right)=\mathcal{C}(\rho)$.

This construction is two-step. First, for each $v \in V$, we map the subsequence $\rho \downarrow_{v}=$ $\left\langle a_{i_{1}}, \ldots, a_{i_{k}}\right\rangle$ into a post-unique sequence of actions $\rho_{v}^{*}=\left\langle a_{i_{1}}^{*}, \ldots, a_{i_{k}}^{*}\right\rangle$. Note that the indexes $i_{1}, \ldots, i_{k}$ of the action elements of each $\rho \downarrow_{v}$ are the global indexes of these actions along $\rho$, and exactly the same indexes are used for marking the elements of the constructed sequences $\rho_{v}^{*}$. Having constructed the sequences $\rho_{v_{1}}^{*}, \ldots, \rho_{v_{n}}^{*}$, we then merge them into a single actions sequence $\rho^{*}$, and show that $\rho^{*}$ is a valid plan for $\Pi$. The two properties of $\rho^{*}$ as required above will then hold immediately because $\left|\rho^{*}\right|=|\rho|$, and post-uniqueness of $\rho^{*}$ is implied by the individual post-uniqueness of all its per-variable components $\rho_{v}^{*}$.

The mapping of subsequences $\rho \downarrow_{v}$ of $\rho$ to the desired sequences $\rho_{v}^{*}$ for all variables $v$ is performed top-down, consistently with a topological ordering of the causal graph $C G(\Pi)$. This top-down processing allows us to assume that, when constructing $\rho_{v}^{*}$, the subsequences $\rho_{w}^{*}$ for all $w \in \operatorname{pred}(v)$ are already constructed. Given that, while mapping each $\rho \downarrow_{v}=$ $\left\langle a_{i_{1}}, \ldots, a_{i_{k}}\right\rangle$ to the corresponding $\rho_{v}^{*}$, we distinguish between the following three cases.

(1) The subsequence $\rho \downarrow_{v}$ is already post-unique.

In this case, we simply set $\rho_{v}^{*}$ to $\rho \downarrow_{v}$. In addition, we construct the following sets of ordering constraints. First, we set a binary relation $>_{v}$ over the action elements of $\rho_{v}^{*}=\left\langle a_{i_{1}}^{*}, \ldots, a_{i_{k}}^{*}\right\rangle$ to

$$
>_{v}=\left\{a_{i}^{*}<a_{j}^{*} \mid a_{i}^{*}, a_{j}^{*} \in \rho_{v}^{*}, i<j\right\} .
$$

It is immediate from Eq. 7 that $>_{v}$ is a strict total order over the elements of $\rho_{v}^{*}$ as $>_{v}$ simply follows the action indexing inherited by $\rho_{v}^{*}$ from plan $\rho$ via $\rho \downarrow_{v}$.

Now, for each $w \in \operatorname{pred}(v)$, we set a binary relation $>_{v, w}$ over the elements of $\rho_{v}^{*}$ and $\rho_{w}^{*}$ to

$$
>_{v, w}=\left\{\begin{array}{ll}
\bigcup_{a_{i}^{*} \in \rho_{v}^{*}, a_{j}^{*} \in \rho_{w}^{*}}\left\{a_{i}^{*}<a_{j}^{*} \mid i<j\right\} \cup\left\{a_{j}^{*}<a_{i}^{*} \mid j<i\right\}, & \text { pre }(a)[w] \text { is specified for some } a \in \rho_{v}^{*} \\
\emptyset, & \text { otherwise }
\end{array} .\right.
$$


For each $w \in \operatorname{pred}(v)$, the relation $>_{v, w}$ defined by Eq. 8 is a strict total order over its domain because the ordering constraints between the elements of $\rho_{v}^{*}$ and $\rho_{w}^{*}$ are a subset of the constraints induced by the total-order plan $\rho$ over the (corresponding) actions from $\rho \downarrow_{v}$ and $\rho \downarrow_{w}$. For the same reason, from Eqs. 7 and 8, we have that, for each $w \in \operatorname{pred}(v),>_{v} \cup>_{v, w}$ is a strict total order over the union of the elements of $\rho_{v}^{*}$ and $\rho_{w}^{*}$.

From Eqs. 7-8 we can now derive that any linearization of $>_{v} \cup \bigcup_{w \in \operatorname{pred}(v)}>_{v, w}$ defines a sequence of actions that is applicable with respect to $\{v\} \cup \operatorname{pred}(v)$. In addition, $\left|\rho_{v}^{*}\right|=\left|\rho \downarrow_{v}\right|$ implies that this action sequence provides to $v$ the value $G[v]$ if the latter is specified.

(2) The subsequence $\rho \downarrow_{v}$ is not post-unique, but the actions in $\rho \downarrow_{v}$ are all prevailed by the value of a single parent $w \in \operatorname{pred}(v)$.

Since $\rho \downarrow_{v}$ is not post-unique, $\rho \downarrow_{v}$ in this case has to contain instances of at least three action types from $\left\{a_{\mathbf{b}_{v} \mid \mathrm{b}_{w}}, a_{\mathbf{b}_{v} \mid \mathrm{w}_{w}}, a_{\mathrm{w}_{v} \mid \mathbf{b}_{w}}, a_{\mathrm{w}_{v} \mid \mathrm{w}_{w}}\right\}$. Thus, in particular, it must be that

(a) $\left|\rho \downarrow_{w}\right| \geq 1$, and

(b) for some $\beta \in\left\{\mathrm{b}_{w}, \mathrm{w}_{w}\right\}$, we have $a_{\mathrm{w}_{v} \mid \beta}, a_{\mathrm{b}_{v} \mid \beta} \in \rho \downarrow_{v}$.

Given that, we set $\rho_{v}^{*}=\left\langle a_{i_{1}}^{*}, \ldots, a_{i_{k}}^{*}\right\rangle$ to

$$
\forall 1 \leq j \leq k: \quad a_{i_{j}}^{*}=\left\{\begin{array}{ll}
a_{\mathrm{w}_{v} \mid \beta}, & j \text { is odd } \\
a_{\mathrm{b}_{v} \mid \beta}, & j \text { is even }
\end{array} .\right.
$$

Both post-uniqueness of such $\rho_{v}^{*}$, as well as its applicability with respect to $v$ are straightforward. The ordering constraints $>_{v}$ are then set according to Eq. 7. Likewise, if $\rho_{w}^{*}=\left\langle a_{j_{1}}, \ldots, a_{j_{l}}\right\rangle$, we set

$$
>_{v, w}= \begin{cases}\bigcup_{a_{i}^{*} \in \rho_{v}^{*}}\left\{a_{i}^{*}<a_{j_{1}}\right\}, & \beta=\mathrm{b}_{w} \\ \bigcup_{a_{i}^{*} \in \rho_{v}^{*}}\left\{a_{i}^{*}>a_{j_{1}}\right\}, & \beta=\mathrm{w}_{w}, l=1 \\ \bigcup_{a_{i}^{*} \in \rho_{v}^{*}}\left\{a_{i}^{*}>a_{j_{1}}\right\} \cup\left\{a_{i}^{*}<a_{j_{2}}\right\}, & \beta=\mathrm{w}_{w}, l>1\end{cases}
$$

Finally, the ordering constraints $>_{v, w^{\prime}}$ for the rest of the parents $w^{\prime} \in \operatorname{pred}(v) \backslash\{w\}$ are set to empty sets.

The relation $>_{v}$ here is identical to this in case (1), and thus it is a strict total order over the elements of $\rho_{v}^{*}$. From Eq. 9, it is easy to verify that $>_{v, w}$ is also a strict partial order over the union of the elements of $\rho_{v}^{*}$ and $\rho_{w}^{*}$. Finally, as all the elements of $\rho_{v}^{*}$ are all identically constrained with respect to the elements of $\rho_{w}^{*}$, we have $>_{v} \cup>_{v, w}$ forming a strict partial order over the union of the elements of $\rho_{v}^{*}$ and $\rho_{w}^{*}$. (For all other parents $w^{\prime} \in \operatorname{pred}(v)$, we simply have $>_{v} \cup>_{v, w}=>_{v}$.)

From Eqs. 7 and 9 we can now derive that any linearization of $>_{v} \cup \bigcup_{w \in \operatorname{pred}(v)}>_{v, w}$ defines a sequence of actions that is applicable with respect to $\{v\} \cup \operatorname{pred}(v)$. In addition, $\left|\rho_{v}^{*}\right|=\left|\rho \downarrow_{v}\right|$ implies that this action sequence provides to $v$ the value $G[v]$ if the latter is specified. 
(3) The subsequence $\rho \downarrow_{v}$ is not post-unique, and the actions of $\rho \downarrow_{v}$ are prevailed by more than one parent of $v$.

The setting of this case in particular implies that there is a pair of $v$ 's parents $\{u, w\} \subseteq$ $\operatorname{pred}(v)$ such that $a_{\mathrm{w}_{v} \mid \alpha}, a_{\mathrm{b}_{v} \mid \beta} \in \rho \downarrow_{v}$ for some $\alpha \in\left\{\mathrm{b}_{u}, \mathrm{w}_{u}\right\}, \beta \in\left\{\mathrm{b}_{w}, \mathrm{w}_{w}\right\}$. Given that, we set $\rho_{v}^{*}$ to

$$
\forall 1 \leq j \leq k: \quad a_{i_{j}}^{*}=\left\{\begin{array}{ll}
a_{\mathrm{w}_{v} \mid \alpha}, & j \text { is odd } \\
a_{\mathrm{b}_{v} \mid \beta}, & j \text { is even }
\end{array},\right.
$$

and, similarly to case (2), both post-uniqueness of $\rho_{v}^{*}$, and its applicability with respect to $v$ are straightforward.

Here as well, the ordering constraints $>_{v}$ are set according to Eq. 7. Likewise, if $\rho_{w}^{*}=\left\langle a_{j_{1}}, \ldots, a_{j_{l}}\right\rangle$, and $\rho_{u}^{*}=\left\langle a_{j_{1}^{\prime}}, \ldots, a_{j_{l^{\prime}}}\right\rangle$, we set $\rangle_{v, w}$ according to Eq. 9 above, and $>_{v, u}$ according to Eq. 10 below.

$$
>_{v, u}= \begin{cases}\bigcup_{a_{i}^{*} \in \rho_{v}^{*}}\left\{a_{i}^{*}<a_{j_{1}^{\prime}}\right\}, & \alpha=\mathrm{b}_{u} \\ \bigcup_{a_{i}^{*} \in \rho_{v}^{*}}\left\{a_{i}^{*}>a_{j_{1}^{\prime}}\right\}, & \alpha=\mathrm{w}_{u}, l^{\prime}=1 \\ \bigcup_{a_{i}^{*} \in \rho_{v}^{*}}\left\{a_{i}^{*}>a_{j_{1}^{\prime}}\right\} \cup\left\{a_{i}^{*}<a_{j_{2}^{\prime}}\right\}, & \alpha=\mathrm{w}_{u}, l^{\prime}>1\end{cases}
$$

Finally, the ordering constraints $>_{v, w^{\prime}}$ for the rest of the parents $w^{\prime} \in \operatorname{pred}(v) \backslash\{u, w\}$ are set to empty sets.

The relation $>_{v}$ here is identical to this in cases (1-2), and relations $>_{v, u}$ and $>_{v, w}$ are effectively identical to the relation $>_{v, w}$ in case (2). Thus, we have $>_{v} \cup>_{v, u}$ and $>_{v} \cup>_{v, w}$ forming strict partial orders over the unions of the elements of $\rho_{v}^{*}$ and $\rho_{u}^{*}$, and $\rho_{v}^{*}$ and $\rho_{w}^{*}$, respectively.

From Eqs. 7, 9, and 10 we can now derive that any linearization of $>_{v} \cup \bigcup_{w \in \operatorname{pred}(v)}>_{v, w}$ defines a sequence of actions that is applicable with respect to $\{v\} \cup \operatorname{pred}(v)$. In addition, $\left|\rho_{v}^{*}\right|=\left|\rho \downarrow_{v}\right|$ implies that this action sequence provides to $v$ the value $G[v]$ if the latter is specified.

As the last step, we now prove that, for each $v \in V$ and each $w \in \operatorname{pred}(v)$, we have $>_{w} \cup>_{v, w}$ being a strict partial order over the union of the elements of $\rho_{w}^{*}$ and $\rho_{v}^{*}$.

- If $>_{v, w}$ is constructed according to Eq. 8, then $>_{w} \cup>_{v, w}$ is a subset of the constraints induced by plan $\rho$ over the (corresponding to $\rho_{v}^{*}$ and $\rho_{w}^{*}$ ) actions from $\rho \downarrow_{v}$ and $\rho_{w}$.

- Otherwise, if $>_{v, w}$ is constructed according to Eq. 9 or (in this case, equivalent) Eq. 10, then $>_{v, w}$ (i) addresses at most two elements of $\rho_{w}$, (ii) orders these elements consistently with $>{ }_{w}$.

In both cases, the argued properties of $>_{w} \cup>_{v, w}$ implies that it forms a strict partial order over the union of the elements of $\rho_{v}^{*}$ and $\rho_{w}^{*}$.

Until now, we have specified the sequences $\rho_{v}^{*}$, the orders $>_{v}$ induced by these sequences, the orders $>_{v, w}$, and proved that all $>_{v} \cup>_{v, w}$ and $>_{w} \cup>_{v, w}$ form strict partial orders 
over their domains. This construction allows us to apply now Theorem 1 to the (considered as sets) sequences $\rho_{v}^{*}$ and orders $>_{v}$ and $>_{v, w}$, proving that

$$
>=\bigcup_{v \in V}\left(>_{v} \cup \bigcup_{w \in \operatorname{pred}(v)}>_{v, w}\right)
$$

forms a strict partial order over the union of $\rho_{v_{1}}^{*}, \ldots, \rho_{v_{n}}^{*}$. Putting thing together, the above implies that any linearization $\rho^{*}$ of $>$ is a plan for $\Pi$, and post-uniqueness of all its subsequences $\rho_{v_{1}}^{*}, \ldots, \rho_{v_{n}}^{*}$ then implies $\rho^{*} \in \mathcal{P}^{\mathrm{pu}}(\Pi)$. Moreover, if $\rho$ is an optimal plan for $\Pi$, then $\left|\rho^{*}\right|=|\rho|$ implies the optimality of $\rho^{*}$.

\subsection{Construction, Correctness, and Complexity}

The main impact of Theorem 5 on our planning-to-COP scheme for uniform-cost $\mathbf{P}(1)$ is that we can now restrict our attention to post-unique plans only. Given that, the constraint optimization problem $\operatorname{COP}_{\Pi}=(\mathcal{X}, \mathcal{F})$ for a uniform-cost problem $\Pi=(V, A, I, G) \in \mathbf{P}(1)$ is specified as follows.

The variable set $\mathcal{X}$ contains a variable $x_{v}$ for each planning variable $v \in V$, and a variable $x_{v}^{w}$ for each edge $(w, v) \in C G(\Pi)$. That is,

$$
\begin{aligned}
\mathcal{X} & =\mathcal{X}^{V} \cup \mathcal{X}^{\mathcal{E}}, \\
\mathcal{X}^{V} & =\left\{x_{v} \mid v \in V\right\} \\
\mathcal{X}^{\mathcal{E}} & =\left\{x_{v}^{w} \mid(w, v) \in C G(\Pi)\right\}
\end{aligned}
$$

For each variable $x_{v} \in \mathcal{X}^{V}$, the domain $\operatorname{Dom}\left(x_{v}\right)$ consists of all goal-valid prefixes of $\sigma(v)$. For each variable $x_{v}^{w} \in \mathcal{X}^{\mathcal{E}}$, the domain $\operatorname{Dom}\left(x_{v}^{w}\right)$ consists of all triples of integers $\left.\llbracket \delta_{\mathrm{w}}, \delta_{\mathrm{b}}, \eta\right]$ satisfying Eq. 12.

$$
\begin{aligned}
& \operatorname{Dom}\left(x_{v}\right)=\unrhd^{*}[\sigma(v)] \\
& \operatorname{Dom}\left(x_{v}^{w}\right)=\left\{\left[\delta_{\mathrm{w}}, \delta_{\mathrm{b}}, \eta\right] \mid \delta_{\mathrm{w}}, \delta_{\mathrm{b}} \in\{0,1\}, 0 \leq \eta \leq n\right\}
\end{aligned}
$$

The semantics of Eq. 12 is as follows. Let $\left\{w_{1}, \ldots, w_{k}\right\}$ be an arbitrary fixed ordering of $\operatorname{pred}(v)$. If $x_{v}$ takes the value $\sigma_{v} \in \operatorname{Dom}\left(x_{v}\right)$, then $v$ is forced to provide the sequence of values $\sigma_{v}$. In turn, if $x_{v}^{w_{i}}$ takes the value $\left.\llbracket \delta_{\mathrm{w}}, \delta_{\mathrm{b}}, \eta\right]$, then $\eta$ corresponds to the number of value changes of $v, \delta_{\mathrm{w}}=1\left(\delta_{\mathrm{b}}=1\right)$ forces the subset of parents $\left\{w_{1}, \ldots, w_{i}\right\} \subseteq \operatorname{pred}(v)$ to support (that is, prevail) all the changes of $v$ to $\mathrm{w}_{v}$ (respectively, to $\mathrm{b}_{v}$ ), and $\delta_{\mathrm{w}}=0$ $\left(\delta_{\mathbf{b}}=0\right)$ relieves this subset of parents $\left\{w_{1}, \ldots, w_{i}\right\}$ from that responsibility.

For each variable $x \in \mathcal{X}$, the set $\mathcal{F}$ contains a non-negative, real-valued function $\varphi_{x}$ with the scope

$$
Q_{x}= \begin{cases}\left\{x_{v}\right\}, & x=x_{v}, k=0 \\ \left\{x_{v}, x_{v}^{w_{k}}\right\}, & x=x_{v}, k>0 \\ \left\{x_{v}^{w_{1}}, x_{w_{1}}\right\}, & x=x_{v}^{w_{1}}, k>0 \\ \left\{x_{v}^{w_{j}}, x_{v}^{w_{j-1}}, x_{w_{j}}\right\}, & x=x_{v}^{w_{j}}, 1<j \leq k\end{cases}
$$

where $\operatorname{pred}(v)=\left\{w_{1}, \ldots, w_{k}\right\}$ (and $k=0$ means $\operatorname{pred}(v)=\emptyset$ ). Proceeding now with specifying these functional components $\mathcal{F}$ of $\mathrm{COP}_{\Pi}$, first, for each $x_{v}$ with $\operatorname{pred}(v)=\emptyset$, and 
for each $\sigma_{v} \in \unrhd^{*}[\sigma(v)]$, we set $\varphi_{x_{v}}\left(\sigma_{v}\right)$ to

$$
\varphi_{x_{v}}\left(\sigma_{v}\right)= \begin{cases}0, & \left|\sigma_{v}\right|=1 \\ 1, & \left(\left|\sigma_{v}\right|=2\right) \wedge\left(a_{\mathrm{w}_{v}} \in A_{v}\right) \\ \left|\sigma_{v}\right|-1, & \left(\left|\sigma_{v}\right|>2\right) \wedge\left(a_{\mathrm{w}_{v}}, a_{\mathrm{b}_{v}} \in A_{v}\right) \\ \infty, & \text { otherwise }\end{cases}
$$

In turn, for each planning variable $v \in V$ with $\operatorname{pred}(v)=\left\{w_{1}, \ldots, w_{k}\right\}, k>0$, the function $\varphi_{x_{v}}$ is set to

$$
\varphi_{x_{v}}\left(\sigma_{v},\left[\delta_{\mathrm{w}}, \delta_{\mathrm{b}}, \eta\right]\right)= \begin{cases}0, & \left(\left|\sigma_{v}\right|=1\right) \wedge\left(\left[\delta_{\mathrm{w}}, \delta_{\mathrm{b}}, \eta\right]=[0,0,0]\right), \\ 1, & \left(\left|\sigma_{v}\right|=2\right) \wedge\left(\left[\delta_{\mathrm{w}}, \delta_{\mathrm{b}}, \eta\right]=[1,0,1]\right), \\ \left|\sigma_{v}\right|-1, & \left(\left|\sigma_{v}\right|>2\right) \wedge\left(\left[\delta_{\mathrm{w}}, \delta_{\mathrm{b}}, \eta\right]=\left[1,1,\left|\sigma_{v}\right|-1\right]\right), \\ \infty, & \text { otherwise }\end{cases}
$$

The functions $\varphi_{x_{v}}$ capture the, marginal over the actions $A_{v}$, cost of providing a sequence $\sigma_{v}$ of value changes of $v$ in $\Pi$, given that (in case of Eq. 15) the parents of $v$ are "ready to support these value changes". In specifying the remaining functional components we use an "indicator" function $\varphi$ specified in Eq. 16.

$$
\varphi\left(\left[\delta_{\mathrm{w}}, \delta_{\mathrm{b}}, \eta\right], \sigma_{w}\right)= \begin{cases}0, & \delta_{\mathrm{w}}=0, \delta_{\mathrm{b}}=0, \\ 0, & \delta_{\mathrm{w}}=1, \delta_{\mathrm{b}}=0,\left(a_{\mathrm{w}_{v} \mid \mathrm{b}_{w}} \in A_{v}\right) \vee\left(\left(\left|\sigma_{w}\right|>1\right) \wedge\left(a_{\mathrm{w}_{v} \mid \mathrm{w}_{w}} \in A_{v}\right)\right), \\ 0, & \delta_{\mathrm{w}}=0, \delta_{\mathrm{b}}=1,\left(a_{\mathrm{b}_{v}} \mid \mathrm{b}_{w} \in A_{v}\right) \vee\left(\left(\left|\sigma_{w}\right|>1\right) \wedge\left(a_{\mathrm{b}_{v}} \mid \mathrm{w}_{w} \in A_{v}\right)\right), \\ 0, & \delta_{\mathrm{w}}=1, \delta_{\mathrm{b}}=1,\left(a_{\mathrm{w}_{v}} \mid \mathrm{b}_{w}, a_{\mathrm{b}_{v} \mid \mathrm{b}_{w}} \in A_{v}\right) \vee\left(\left(\left|\sigma_{w}\right|>1\right) \wedge\left(a_{\mathrm{w}_{v}} \mid \mathrm{w}_{w}, a_{\mathrm{b}_{v} \mid \mathrm{w}_{w}} \in A_{v}\right)\right), \\ 0, & \delta_{\mathrm{w}}=1, \delta_{\mathrm{b}}=1,\left|\sigma_{w}\right| \geq \eta, a_{\mathrm{w}_{v} \mid \mathrm{b}_{w}}, a_{\mathrm{b}_{v}} \mid \mathrm{w}_{w} \in A_{v}, \\ 0, & \delta_{\mathrm{w}}=1, \delta_{\mathrm{b}}=1,\left|\sigma_{w}\right|>\eta, a_{\mathrm{w}_{v}}\left|\mathrm{w}_{w}, a_{\mathrm{b}_{v}}\right| \mathrm{b}_{w} \in A_{v}, \\ \infty, & \text { otherwise }\end{cases}
$$

The semantics of $\varphi$ is that, for each planning variable $v \in V$, each $w \in \operatorname{pred}(v)$, and each $\left(\left[\delta_{\mathrm{w}}, \delta_{\mathrm{b}}, \eta\right], \sigma_{w}\right) \in \operatorname{Dom}\left(x_{v}^{w}\right) \times \operatorname{Dom}\left(x_{w}\right)$, we have $\varphi\left(\left[\delta_{\mathrm{w}}, \delta_{\mathrm{b}}, \eta\right], \sigma_{w}\right)=0$ if the value sequence $\sigma_{w}$ of $w$ can support all the changes of $v$ to $\mathrm{w}_{v}$ (if $\delta_{\mathrm{w}}=1$ ) and all the changes of $v$ to $\mathrm{b}_{v}$ (if $\delta_{\mathrm{b}}=1$ ), out of $\eta$ value changes of $v$ in $\Pi$. Given this indicator function $\varphi$, for each $v \in V$, the functional component $\varphi_{x_{v}}^{w_{1}}$ is specified as

$$
\varphi_{x_{v}^{w_{1}}}\left(\left[\delta_{\mathrm{w}}, \delta_{\mathrm{b}}, \eta\right], \sigma_{w_{1}}\right)=\varphi\left(\left[\delta_{\mathrm{w}}, \delta_{\mathrm{b}}, \eta\right], \sigma_{w_{1}}\right),
$$

and the rest of the functions $\varphi_{x_{v}}^{w_{2}}, \ldots, \varphi_{x_{v}}^{w_{k}}$ are specified as follows. For each $2 \leq i \leq k$, the value of the function $\varphi_{x_{v}}^{w_{j}}$ at the combination of $\left[\delta_{\mathrm{w}}, \delta_{\mathrm{b}}, \eta\right] \in \operatorname{Dom}\left(x_{v}^{w_{i}}\right), \llbracket\left[\delta_{\mathrm{w}}^{\prime}, \delta_{\mathrm{b}}^{\prime}, \eta^{\prime} \rrbracket \in\right.$ $\operatorname{Dom}\left(x_{v}^{w_{i-1}}\right)$, and $\sigma_{w_{i}} \in \operatorname{Dom}\left(x_{w_{i}}\right)=\unrhd^{*}\left[\sigma\left(w_{i}\right)\right]$ is specified as

$$
\left.\varphi_{x_{v}^{w_{i}}}\left(\left[\delta_{\mathrm{w}}, \delta_{\mathrm{b}}, \eta\right], \llbracket \delta_{\mathrm{w}}^{\prime}, \delta_{\mathrm{b}}^{\prime}, \eta^{\prime}\right], \sigma_{w_{j}}\right)= \begin{cases}\varphi\left(\left[\delta_{\mathrm{w}}-\delta_{\mathrm{w}}^{\prime}, \delta_{\mathrm{b}}-\delta_{\mathrm{b}}^{\prime}, \eta\right], \sigma_{w_{j}}\right), & \eta=\eta^{\prime} \wedge \delta_{\mathrm{w}} \geq \delta_{\mathrm{w}}^{\prime} \wedge \delta_{\mathrm{b}} \geq \delta_{\mathrm{b}}^{\prime} \\ \infty & \text { otherwise }\end{cases}
$$




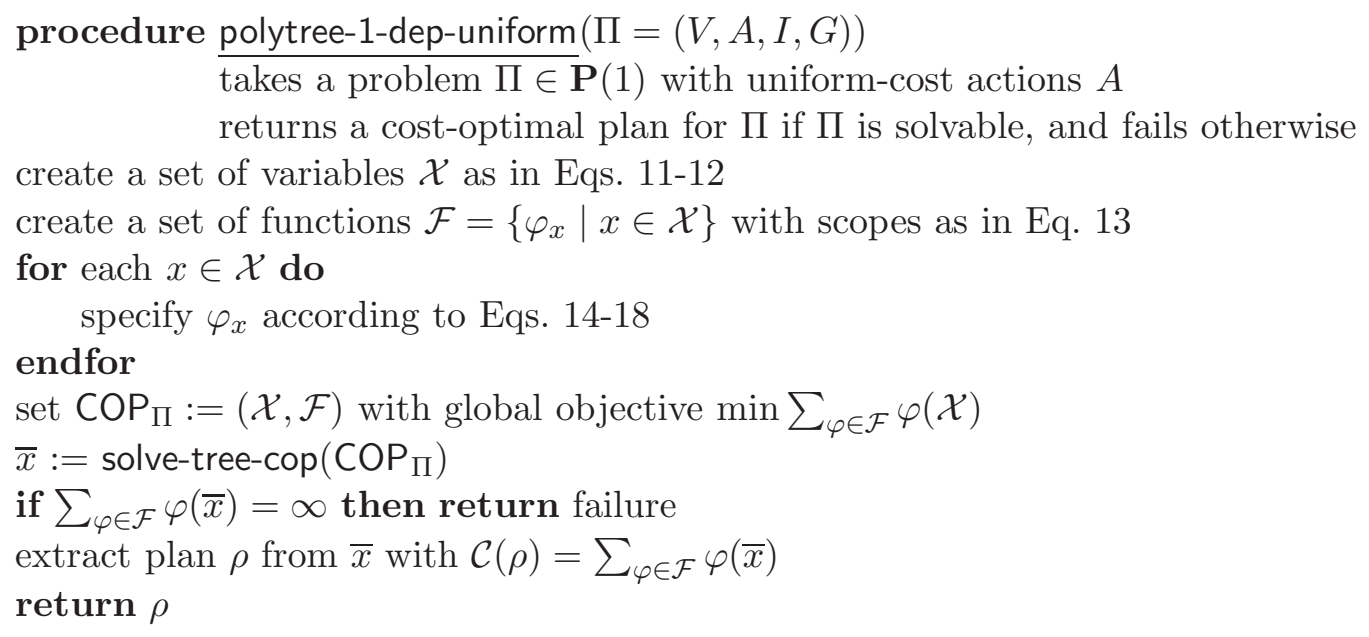

Figure 10: Algorithm for cost-optimal planning for $\mathbf{P}(1)$ problems with uniform-cost actions.

This finalizes the construction of $\mathrm{COP}_{\Pi}$, and this construction constitutes the first three steps of the algorithm polytree-1-dep-uniform in Figure 10(a). The subsequent steps of this algorithm are conceptually similar to these of the polytree-k-indegree algorithm in Section 3, with the major difference being in the plan reconstruction routines. It is not hard to verify from Eqs. 11-13, and the fact that the causal graph of $\Pi \in \mathbf{P}(1)$ forms a polytree that

(i) for each variable $x \in \mathcal{X},|\operatorname{Dom}(x)|=\operatorname{poly}(n)$,

(ii) the tree-width of the cost network of $\mathcal{F}$ is $\leq 3$, and

(iii) the optimal tree-decomposition of the $\mathrm{COP}_{\Pi}$ 's cost network is given by any topological ordering of the causal graph that is consistent with the (arbitrary yet fixed at the time of the $\mathrm{COP}_{\Pi}$ 's construction) orderings of each planning variable's parents in the causal graph.

For an illustration, we refer the reader to Figure 4 (p. 211) where Figure 4(a) depicts the causal graph of a problem $\Pi \in \mathbf{P}(1)$, and Figure 4(c) depicts the cost network of the corresponding $\mathrm{COP}_{\Pi}$. The top-most variables and the cliques in the cost network correspond to the functional components of $\mathrm{COP}_{\Pi}$.

We now proceed with proving the correctness and complexity of the polytree-1-depuniform algorithm.

Theorem 6 Let $\Pi$ be a $\mathbf{P}(1)$ problem with uniform-costs actions, $\operatorname{COP}_{\Pi}=(\mathcal{X}, \mathcal{F})$ be the corresponding constraint optimization problem, and $\bar{x}$ be an optimal assignment to $\mathcal{X}$ with $\sum_{\varphi \in \mathcal{F}} \varphi(\bar{x})=\alpha$.

(I) If $\alpha<\infty$, then a plan of cost $\alpha$ for $\Pi$ can be reconstructed from $\bar{x}$ in time polynomial in the description size of $\Pi$.

(II) If $\Pi$ has a plan, then $\alpha<\infty$. 
Proof Sketch: The proof of Theorem 6 is in Appendix A. p. 249. The overall flow of the proof is similar to this of the proof of Theorem 2, yet the details are very much different. The main source of the proof's complexity is that, in proving (I), we must distinguish between several cases based on the roles taken by the (up to) two parents supporting the value changes of each variable in question.

Theorem 7 Cost-optimal planning for $\mathbf{P}(1)$ with uniform cost actions is tractable.

Proof: Given a planning problem $\Pi \in \mathbf{P}(1)$ with uniform cost actions, we show that the corresponding constraint optimization problem $\mathrm{COP}_{\Pi}$ can be constructed and solved in time polynomial in the description size of $\Pi$.

Let $n$ be the number of state variables in $\Pi$. In polytree-1-dep-uniform, we first construct the constraint optimization problem $\operatorname{COP}_{\Pi}$ over $\Theta\left(n^{2}\right)$ variables $\mathcal{X}$ with domain sizes bounded by $O(n)$, and $\Theta\left(n^{2}\right)$ functional components $\mathcal{F}$, each defined over at most three COP variables. The construction is linear in the size of the resulting COP, and thus is accomplished in time $O\left(n^{5}\right)$.

Applying then to $\mathrm{COP}_{\Pi}$ a tree-decomposition along the scopes of the functional components $\mathcal{F}$, we arrive into an equivalent, tree-structured constraint optimization problem over $\Theta\left(n^{2}\right)$ variables with domains of size $O\left(n^{3}\right)$. Such a tree-structured COP can be solved in time $O\left(x y^{2}\right)$ where $x$ is the number of variables and $y$ is an upper bound on the size of a variable's domain (Dechter, 2003). Therefore, solving $\mathrm{COP}_{\Pi}$ can be done in time $O\left(n^{8}\right)$. As this dominates both the time complexity of constructing $\mathrm{COP}_{\Pi}$, and the time complexity of extracting a plan from the optimal solution to $\mathrm{COP}_{\Pi}$ (see the proof of (I) in Theorem 6), the overall complexity of the algorithm polytree-1-dep-uniform is $O\left(n^{8}\right)$, and therefore polynomial in the description size of $\Pi$.

\section{Cost-Optimal Planning for $\mathrm{P}(1)$ with General Action Costs}

We now consider cost-optimal planning for $\mathbf{P}(1)$ problems without the constraints on actions to be all of the same cost. While Theorem 5 in Section 4 shows that any solvable $\mathbf{P}(1)$ problem $\Pi$ has at least one post-unique plan, it is possible that no such plan is cost-optimal for $\Pi$, and Example 1 below affirms this possibility.

Example 1 Let $\Pi=\langle V, A, I, G\rangle$ be the $\mathbf{P}(1)$ problem instance over variables $V=\left\{v_{1}, \ldots, v_{5}\right\}$, $I=\{0,0,0,0,0\}, G=\left\{v_{1}=0, v_{2}=1, v_{3}=1\right\}$, and actions $A$ as depicted in Figure 11a. The polytree causal graph of $\Pi$ is shown in Figure 11b, and it is easy verify from the table in Figure 11a that $\Pi \in \mathbf{P}(1)$.

Ignoring the non-uniformity of the action costs, this problem has a post-unique costoptimal plan $\rho=\left\langle a_{4} \cdot a_{2} \cdot a_{1} \cdot a_{5} \cdot a_{3} \cdot a_{4}\right\rangle$. However, considering the action costs as in the last column in Figure 11a, then the cost of each optimal plan, such as $\rho^{\prime}=\left\langle a_{4} \cdot a_{2} \cdot a_{1} \cdot a_{5} \cdot a_{3} \cdot a_{7} \cdot a_{6}\right\rangle$ will be $\mathcal{C}\left(\rho^{\prime}\right)=16<24=\mathcal{C}(\rho)$. Note that the plan $\rho^{\prime}$ is not post-unique because it changes the value of $v_{3}$ to 1 using both actions $a_{4}$ and $a_{6}$. In fact, any plan for this problem will have at least two action instances that change the value of $v_{3}$ to 1 . However, the cheap such action $a_{6}$ cannot be applied twice because it requires $v_{4}=1$, and the only action $a_{5}$ that sets $v_{3}=0$ cannot be applied after $a_{6}-a_{5}$ requires $v_{4}=0$, and there is no action that has 


\begin{tabular}{|c||c|c|c|c|c||c|c|c|c|c||c|}
\hline \multicolumn{1}{|c||}{$A$} & \multicolumn{6}{c||}{$\operatorname{pre}(a)$} & \multicolumn{5}{c|}{$\operatorname{eff}(a)$} \\
\hline & $v_{1}$ & $v_{2}$ & $v_{3}$ & $v_{4}$ & $v_{5}$ & $v_{1}$ & $v_{2}$ & $v_{3}$ & $v_{4}$ & $v_{5}$ & \\
\hline \hline$a_{1}$ & 1 & 0 & & & & & 1 & & & & 1.0 \\
\hline$a_{2}$ & 0 & & 1 & & & 1 & & & & & 1.0 \\
\hline$a_{3}$ & 1 & & 0 & & & 0 & & & & & 1.0 \\
\hline$a_{4}$ & & & 0 & & 0 & & & 1 & & & 10.0 \\
\hline$a_{5}$ & & & 1 & 0 & & & & 0 & & & 1.0 \\
\hline$a_{6}$ & & & 0 & 1 & & & & 1 & & & 1.0 \\
\hline$a_{7}$ & & & & 0 & & & & & 1 & & 1.0 \\
\hline \hline
\end{tabular}

(a)

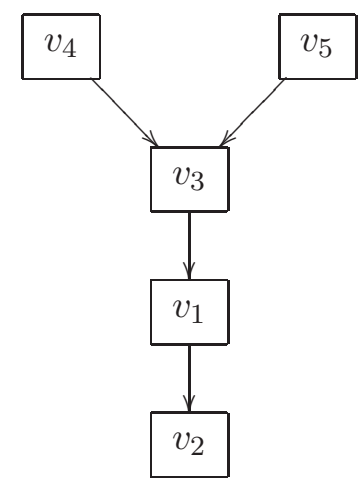

(b)

Figure 11: Action set and the causal graph for the problem in Example 1.

this effect. Therefore, any post-unique plan for this problem will have to invoke twice the action $a_{4}$, and thus will have cost of at least $2 \cdot \mathcal{C}\left(a_{4}\right)=20$.

Fortunately, we show that any solvable $\mathbf{P}(1)$ problem is guaranteed to have a costoptimal plan satisfying a certain "relaxation" of action sequence post-uniqueness that still allows us to devise a (more costly than polytree-1-dep-uniform) planning-to-COP scheme for general $\mathbf{P}(1)$ problems.

\subsection{Post-3/2 Plans and P(1) Problems}

We now proceed with introducing the notion of post-3/2 property for action sequences that relaxes the post-uniqueness property exploited in the previous section.

Definition 5 Let $\Pi=(V, A, I, G)$ be a UB problem instance. An action sequence $\varrho \in A^{*}$ is called post-3/2 if, for each $v \in V, a \in \varrho \downarrow_{v}$, there exist $\alpha \neq \beta \in\left\{\mathrm{b}_{v}, \mathrm{w}_{v}\right\}$, a parent $w \in \operatorname{pred}(v), \gamma, \delta \in\left\{\mathrm{b}_{w}, \mathrm{w}_{w}\right\}$, and $\xi \in\left\{\mathrm{b}_{u}, \mathrm{w}_{u} \mid u \in \operatorname{pred}(v)\right\}$, such that $a \in\left\{a_{\alpha \mid \gamma}, a_{\beta \mid \delta}, a_{\alpha \mid \xi}\right\}$. That is, all the changes of each variable are done using at most three types of actions which are prevailed by at most two parents, and if $u$ is different from $w$, then different actions prevailed by $w$ perform different value changes of $v$.

The (possibly empty) set of all post-3/2 plans for $\Pi$ is denoted by $\mathcal{P}^{3 / 2}(\Pi)$ (or simply $\mathcal{P}^{3 / 2}$, if the identity of $\Pi$ is clear from the context).

To illustrate the rather involved definition of post-3/2 plans, consider the following four action sequences of four actions each. The only value changes made by these sequences are changes of variable $v$ with $\operatorname{pred}(v)=\{x, y, z\}$.

- $\left\langle a_{\mathrm{w}_{v} \mid \mathbf{b}_{x}} \cdot a_{\mathbf{b}_{v} \mid \mathrm{w}_{x}} \cdot a_{\mathrm{w}_{v} \mid \mathbf{b}_{x}} \cdot a_{\mathbf{b}_{v} \mid \mathbf{b}_{y}}\right\rangle$ is post-3/2 because it uses three types of actions, each prevailed by one of the two parents $x, y$.

- $\left\langle a_{\mathrm{w}_{v} \mid \mathbf{b}_{x}} \cdot a_{\mathbf{b}_{v} \mid \mathbf{b}_{y}} \cdot a_{\mathrm{w}_{v} \mid \mathbf{b}_{x}} \cdot a_{\mathbf{b}_{v} \mid \mathbf{b}_{y}}\right\rangle$ is post-3/2 because it uses two types of actions, each prevailed by one of the two parents $x, y$. 
- $\left\langle a_{\mathrm{w}_{v} \mid \mathbf{b}_{x}} \cdot a_{\mathbf{b}_{v} \mid \mathbf{b}_{y}} \cdot a_{\mathrm{w}_{v} \mid \mathbf{b}_{x}} \cdot a_{\mathbf{b}_{v} \mid \mathbf{b}_{z}}\right\rangle$ is not post-3/2 because it uses three types of actions, each prevailed by one of the three parents $x, y, z$.

- $\left\langle a_{\mathrm{w}_{v} \mid \mathbf{b}_{x}} \cdot a_{\mathrm{b}_{v} \mid \mathrm{w}_{x}} \cdot a_{\mathrm{w}_{v} \mid \mathrm{b}_{y}} \cdot a_{\mathrm{b}_{v} \mid \mathrm{b}_{y}}\right\rangle$ is not post-3/2 because it uses four types of actions, each prevailed by one of the two parents $x, y$.

It is not hard to verify that post-3/2 is a relaxation of post-uniqueness - if a plan is post-unique, then it is post- $3 / 2$, but not necessarily the other way around. Turns out that, for any $\mathbf{P}(1)$ problem $\Pi$, this relaxed property is guaranteed to be satisfied by at least one cost-optimal plan for $\Pi$.

Theorem 8 For every solvable $\mathbf{P}(1)$ problem $\Pi=(V, A, I, G)$, the plan set $\mathcal{P}^{3 / 2}(\Pi)$ contains at least one cost-optimal plan.

Proof Sketch: The proof of Theorem 8 is in Appendix A, pp. 255-278. This proof is flow-wise similar to the proof of Theorem 5, but is technically much more involved. Here we provide only a sketch of the proof. We note, however, that many building blocks of this proof are then used in the correctness proof for our planning-to-COP algorithm (notably, in Theorem 9).

Given a $\mathbf{P}(1)$ problem $\Pi=(V, A, I, G)$, and cost-optimal plan $\rho$ for $\Pi$, we construct a post-3/2 plan $\rho^{*}$ for $\Pi$, such that $\mathcal{C}\left(\rho^{*}\right)=\mathcal{C}(\rho)$. In nutshell, first, for each $v \in V$, we map the subsequence $\rho \downarrow_{v}=\left\langle a_{1}, \ldots, a_{k}\right\rangle$ of $\rho$ into a sequence of actions $\rho_{v}^{*}=\left\langle a_{1}^{*}, \ldots, a_{k}^{*}\right\rangle$ that (i) satisfy the post-3/2 property, and (ii) $\mathcal{C}\left(\rho_{v}^{*}\right) \leq \mathcal{C}\left(\rho \downarrow_{v}\right)$. Then, we merge the constructed sequences $\left\{\rho_{v}^{*}\right\}_{v \in V}$ into $\rho^{*}$, and show that $\rho^{*}$ is a valid plan for $\Pi$. The two properties of $\rho^{*}$ as required above will then hold immediately because $\mathcal{C}\left(\rho^{*}\right)=\mathcal{C}(\rho)$, and $\rho^{*}$ being post-3/2 is implied by all its per-variable components $\rho_{v}^{*}$ being post- $3 / 2$.

For each variable $v \in V$, with $\operatorname{pred}(v)=\emptyset$, we set $\rho_{v}^{*}=\rho \downarrow_{v}$. In turn, for each variable $v \in V$ with $\operatorname{pred}(v) \neq \emptyset$, given $\left\{\sigma_{w}\right\}_{w \in \operatorname{pred}(v)}$, such that $\left|\sigma_{w}\right|=\left|\rho \downarrow_{w}\right|+1$, let $a_{i}^{\alpha}$ be the $i$ 'th cheapest action that changes variable $v$ to $\alpha \in\left\{\mathrm{b}_{v}, \mathrm{w}_{v}\right\}$ and prevailed by some value from $\left\{\sigma_{w}\right\}_{w \in \operatorname{pred}(v)}$ (that is, applicable given the sequences of values $\left\{\sigma_{w}\right\}_{w \in \operatorname{pred}(v)}$ respectively obtained by the parents of $v$ ). The proof considers (in groups) all possible settings for $a_{1}^{\mathrm{w}}=a_{\mathrm{w}_{v} \mid \gamma}, a_{2}^{\mathrm{w}}=a_{\mathrm{w}_{v} \mid \mu}, a_{1}^{\mathrm{b}}=a_{\mathrm{b}_{v} \mid \delta}, a_{2}^{\mathrm{b}}=a_{\mathrm{b}_{v} \mid \nu}$ (that is, all possible combinations of $\left.\{\gamma, \mu, \delta, \nu\} \subseteq \bigcup_{w \in \operatorname{pred}(v)}\left\{\mathrm{b}_{w}, \mathrm{w}_{w}\right\}\right)$ on a case-by-case basis. Specifically, the cases correspond to

(I) $\gamma=\delta \in\left\{\mathrm{b}_{w}, \mathrm{w}_{w}\right\}$.

(II) $\gamma \in\left\{\mathrm{b}_{w}, \mathrm{w}_{w}\right\}, \delta \in\left\{\mathrm{b}_{u}, \mathrm{w}_{u}\right\}$, such that $w \neq u$.

(III) $\gamma=\mathrm{b}_{w}, \delta=\mathrm{w}_{w}$; here we distinguish between a few cases based on $\sigma_{w}$ and $\sigma_{v}$.

(1) $\left|\rho \downarrow_{v}\right|=2 y+1,\left|\sigma_{w}\right|=2 x,\left|\sigma_{w}\right| \leq\left|\rho \downarrow_{v}\right|$.

(2) $\left|\rho \downarrow_{v}\right|=2 y+1,\left|\sigma_{w}\right|=2 x,\left|\sigma_{w}\right|>\left|\rho \downarrow_{v}\right|$.

(3) $\left|\rho \downarrow_{v}\right|=2 y,\left|\sigma_{w}\right|=2 x,\left|\sigma_{w}\right|<\left|\rho \downarrow_{v}\right|$.

(4) $\left|\rho \downarrow_{v}\right|=2 y,\left|\sigma_{w}\right|=2 x,\left|\sigma_{w}\right| \geq\left|\rho \downarrow_{v}\right|$.

(5) $\left|\rho \downarrow_{v}\right|=2 y+1,\left|\sigma_{w}\right|=2 x+1,\left|\sigma_{w}\right|<\left|\rho \downarrow_{v}\right|$.

(6) $\left|\rho \downarrow_{v}\right|=2 y+1,\left|\sigma_{w}\right|=2 x+1,\left|\sigma_{w}\right| \geq\left|\rho \downarrow_{v}\right|$. 
(7) $\left|\rho \downarrow_{v}\right|=2 y,\left|\sigma_{w}\right|=2 x+1,\left|\sigma_{w}\right| \leq\left|\rho \downarrow_{v}\right|$.

(8) $\left|\rho \downarrow_{v}\right|=2 y,\left|\sigma_{w}\right|=2 x+1,\left|\sigma_{w}\right|>\left|\rho \downarrow_{v}\right|$.

(IV) $\gamma=\mathrm{w}_{w}, \delta=\mathrm{b}_{w}$; here as well we distinguish between eight cases that specification-wise almost identical to these of (III), but lead to different settings of $\rho_{v}^{*}$.

\subsection{Construction, Correctness, and Complexity}

Given a post-3/2 action sequence $\varrho$ from $A$ and a variable $v \in V$, we can distinguish between the following exhaustive roles of each parent $w \in \operatorname{pred}(v)$ with respect to $v$ along $\varrho$.

R1 All the actions in $\varrho$ that change the value of $v$ are supported by the same value of $w$. That is, for some $\gamma \in\left\{\mathrm{b}_{w}, \mathrm{w}_{w}\right\}$, if $a \in \varrho_{v}$, then $a \in\left\{a_{\mathrm{b}_{v} \mid \gamma}, a_{\mathrm{w}_{v} \mid \gamma}\right\}$.

R2 All the actions in $\varrho$ that change the value of $v$ to $\mathrm{w}_{v}$ are supported by the same value of $w$, and all the actions in $\varrho$ that change the value of $v$ to $\mathrm{b}_{v}$ are supported by another value of $w$.

That is, for some $\gamma \neq \delta \in\left\{\mathrm{b}_{w}, \mathrm{w}_{w}\right\}$, if $a \in \varrho_{v}$, then $a \in\left\{a_{\mathrm{b}_{v} \mid \gamma}, a_{\mathrm{w}_{v} \mid \delta}\right\}$.

R3 All the actions in $\varrho$ that change the value of $v$ to $\mathrm{w}_{v}$ are supported by the same value of $w$, and none of the actions in $\varrho$ that change the value of $v$ to $\mathrm{b}_{v}$ are supported by $w$. That is, for some $\gamma \in\left\{\mathrm{b}_{w}, \mathrm{w}_{w}\right\}$ and $\delta \notin\left\{\mathrm{b}_{w}, \mathrm{w}_{w}\right\}$, if $a \in \varrho_{v}$, then $a \in\left\{a_{\mathrm{b}_{v} \mid \delta}, a_{\mathrm{w}_{v} \mid \gamma}\right\}$.

R4 All the actions in $\varrho$ that change the value of $v$ to $\mathrm{b}_{v}$ are supported by the same value of $w$, and none of the actions in $\varrho$ that change the value of $v$ to $\mathbf{w}_{v}$ are supported by $w$. That is, for some $\gamma \in\left\{\mathrm{b}_{w}, \mathrm{w}_{w}\right\}$ and $\delta \notin\left\{\mathrm{b}_{w}, \mathrm{w}_{w}\right\}$, if $a \in \varrho_{v}$, then $a \in\left\{a_{\mathrm{b}_{v} \mid \gamma}, a_{\mathrm{w}_{v} \mid \delta}\right\}$.

R5 All the actions in $\varrho$ that change the value of $v$ to $\mathrm{w}_{v}$ are supported by the same value of $w$, and all the actions in $\varrho$ that change the value of $v$ to $\mathrm{b}_{v}$ are supported by two values of $w$.

That is, for some $\gamma \neq \delta \in\left\{\mathrm{b}_{w}, \mathrm{w}_{w}\right\}$, if $a \in \varrho_{v}$, then $a \in\left\{a_{\mathrm{w}_{v} \mid \gamma}, a_{\mathrm{b}_{v} \mid \delta}, a_{\mathrm{b}_{v} \mid \gamma}\right\}$.

R6 All the actions in $\varrho$ that change the value of $v$ to $\mathrm{b}_{v}$ are supported by the same value of $w$, and all the actions in $\varrho$ that change the value of $v$ to $\mathrm{w}_{v}$ are supported by two values of $w$.

That is, for some $\gamma \neq \delta \in\left\{\mathrm{b}_{w}, \mathrm{w}_{w}\right\}$, if $a \in \varrho_{v}$, then $a \in\left\{a_{\mathrm{b}_{v} \mid \gamma}, a_{\mathrm{w}_{v} \mid \delta}, a_{\mathrm{w}_{v} \mid \gamma}\right\}$.

R7 All the actions in $\varrho$ that change the value of $v$ to $\mathrm{w}_{v}$ are supported by the same value of $w$, and some of the actions in $\varrho$ that change the value of $v$ to $\mathrm{b}_{v}$ are supported by another value of $w$ and others are supported by another parent.

That is, for some $\gamma \neq \delta \in\left\{\mathrm{b}_{w}, \mathrm{w}_{w}\right\}$ and $\mu \notin\left\{\mathrm{b}_{w}, \mathrm{w}_{w}\right\}$, if $a \in \varrho_{v}$, then $a \in\left\{a_{\mathrm{w}_{v} \mid \gamma}, a_{\mathrm{b}_{v} \mid \delta}, a_{\mathrm{b}_{v} \mid \mu}\right\}$.

R8 All the actions in $\varrho$ that change the value of $v$ to $\mathrm{b}_{v}$ are supported by the same value of $w$, and some of the actions in $\varrho$ that change the value of $v$ to $\mathrm{w}_{v}$ are supported by another value of $w$ and others are supported by another parent.

That is, for some $\gamma \neq \delta \in\left\{\mathrm{b}_{w}, \mathrm{w}_{w}\right\}$ and $\mu \notin\left\{\mathrm{b}_{w}, \mathrm{w}_{w}\right\}$, if $a \in \varrho_{v}$, then $a \in\left\{a_{\mathrm{b}_{v} \mid \gamma}, a_{\mathrm{w}_{v} \mid \delta}, a_{\mathrm{w}_{v} \mid \mu}\right\}$. 
R9 Part of the actions in $\varrho$ that change the value of $v$ to $\mathrm{b}_{v}$ are supported by the same value of $w$, and none of the actions in $\varrho$ that change the value of $v$ to $\mathrm{w}_{v}$ are supported by the same value of $w$.

R10 Part of the actions in $\varrho$ that change the value of $v$ to $\mathrm{w}_{v}$ are supported by the same value of $w$, and none of the actions in $\varrho$ that change the value of $v$ to $\mathrm{b}_{v}$ are supported by the same value of $w$.

R11 None of the actions in $\varrho$ are supported by $w$.

That is, if $a_{\alpha \mid \gamma} \in \varrho$, then $\gamma \notin\left\{\mathrm{b}_{w}, \mathrm{w}_{w}\right\}$.

For a given post-3/2 action sequence $\varrho$ from $A$ and a variable $v \in V$, each parent of $v$ performs one of the roles R1-R11 with respect to $v$ along $\varrho$, and each of the roles R1-R10 is performed by at most one of the parents of $v$. In addition, there are sets of roles that cannot be simultaneously performed by the parents of $v$ with respect to $v$ and the same action sequence $\varrho$, and there are roles that have to be performed in pairs. Specifically,

- If one of the roles $\{\mathrm{R} 1, \mathrm{R} 2, \mathrm{R} 5, \mathrm{R} 6\}$ is played by some parent $w^{\prime} \in \operatorname{pred}(v)$, then $\mathrm{R} 11$ must be played by all other parents $w \in \operatorname{pred}(v) \backslash\left\{w^{\prime}\right\}$.

- If $\mathrm{R} 3 / \mathrm{R} 7 / \mathrm{R} 8$ is played by some parent $w_{1} \in \operatorname{pred}(v)$, then $\mathrm{R} 4 / \mathrm{R} 9 / \mathrm{R} 10$, respectively, must be played by some parent $w_{2} \in \operatorname{pred}(v) \backslash\left\{w_{1}\right\}$, and R11 must be played by all other parents $w \in \operatorname{pred}(v) \backslash\left\{w_{1}, w_{2}\right\}$.

Considering a variable $v$ and its parents $\operatorname{pred}(v)$ through the lens of these eleven roles, suppose we now aim at assigning these roles to $\operatorname{pred}(v)$ by considering them one after another in some arbitrary order. Given the aforementioned constraints on the role assignment, at each step of this sequential process we can be in one of the following eight states, with the whole process being described by a state machine depicted in Fig. 12.

S1 All the roles R1-R11 are still available (to be assigned to the parents of $v$ ).

S2 Only roles $\{\mathrm{R} 3, \mathrm{R} 11\}$ are still available.

S3 Only roles $\{\mathrm{R} 4, \mathrm{R} 11\}$ are available.

S4 Only roles $\{\mathrm{R} 7, \mathrm{R} 11\}$ are available.

S5 Only roles $\{\mathrm{R} 8, \mathrm{R} 11\}$ are available.

S6 Only roles $\{\mathrm{R} 9, \mathrm{R} 11\}$ are available.

S7 Only roles $\{\mathrm{R} 10, \mathrm{R} 11\}$ are available.

S8 Only role R11 is available.

Given this language of "roles" and "states", we now proceed with specifying our constraint optimization problem $\mathrm{COP}_{\Pi}=(\mathcal{X}, \mathcal{F})$ for a problem $\Pi=(V, A, I, G) \in \mathbf{P}(1)$. In what follows, for each variable $v \in V$, we assume a fixed (arbitrarily chosen) numbering $\left\{w_{1}, \ldots, w_{k}\right\}$ of $\operatorname{pred}(v)$ with respect to $v$. 


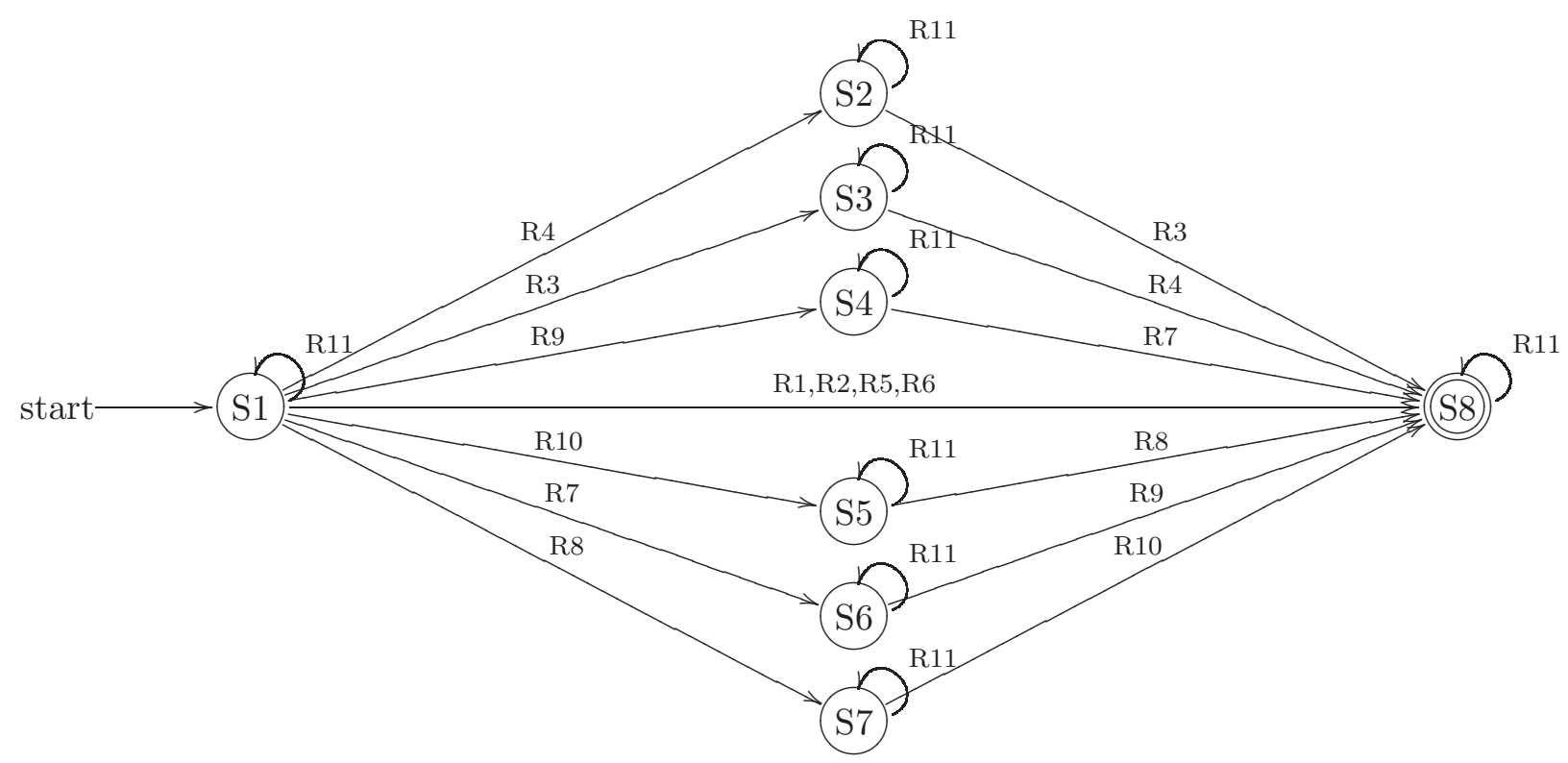

Figure 12: State machine describing the process of "sequential" role assignment to the parents of $v$ (with respect to $v$ ). Each transition is labeled with a set of roles, one of which is getting assigned to a parent of $v$ at the corresponding step.

1. Similarly to the uniform-cost case, the variable set $\mathcal{X}$ contains a variable $x_{v}$ for each planning variable $v \in V$, and a variable $x_{v}^{w}$ for each edge $(w, v) \in C G(\Pi)$. That is,

$$
\begin{aligned}
\mathcal{X} & =\mathcal{X}^{V} \cup \mathcal{X}^{\mathcal{E}} \\
\mathcal{X}^{V} & =\left\{x_{v} \mid v \in V\right\} \\
\mathcal{X}^{\mathcal{E}} & =\left\{x_{v}^{w} \mid(w, v) \in C G(\Pi)\right\}
\end{aligned}
$$

2. For each variable $x_{v} \in \mathcal{X}^{V}$, the domain $\operatorname{Dom}\left(x_{v}\right)$ consists of all possible valid prefixes of $\sigma(v)$. For each variable $x_{v}^{w_{i}} \in \mathcal{X}^{\mathcal{E}}$, the domain $\operatorname{Dom}\left(x_{v}^{w_{i}}\right)$ consists of all possible quadruples satisfying Eq. 20.

$$
\begin{aligned}
\operatorname{Dom}\left(x_{v}\right) & =\left\{\sigma_{v} \in \unrhd^{*}[\sigma(v)]\right\} \\
\operatorname{Dom}\left(x_{v}^{w_{i}}\right) & =\left\{\begin{array}{l|l}
{\left[\mathbf{S}, \#_{\mathrm{w}}, \#_{\mathrm{b}}, \eta\right]} & \begin{array}{l}
0 \leq \eta \leq n, 0 \leq \#_{\mathrm{w}}, \#_{\mathrm{b}} \leq\left\lceil\frac{\eta}{2}\right\rceil \\
\mathbf{S} \in\{\mathrm{S} 1, \ldots, \mathrm{S} 8\}
\end{array}
\end{array}\right\}
\end{aligned}
$$

The semantics of Eq. 20 is as follows. Let $\left\{w_{1}, \ldots, w_{k}\right\}$ be an arbitrary fixed ordering of $\operatorname{pred}(v)$. If $x_{v}$ takes the value $\sigma_{v} \in \operatorname{Dom}\left(x_{v}\right)$, then $v$ is forced to provide the sequence of values $\sigma_{v}$. In turn, if $x_{v}^{w_{i}}$ takes the value $\left[\mathbf{S}, \#_{\mathbf{w}}, \#_{\mathbf{b}}, \eta\right]$, then $\eta$ corresponds to the number of value changes of $v, \#_{\mathrm{w}}$ and $\#_{\mathrm{b}}$ correspond to the number of value changes of $v$ to $\mathbf{w}_{v}$ and $\mathrm{b}_{v}$, respectively, that should be performed by the actions prevailed by the values of 
$\left\{w_{1}, \ldots, w_{i}\right\}$, and the state-component $\mathbf{S}$ captures the roles that can be assigned to the parents $\left\{w_{1}, \ldots, w_{i}\right\}$.

3. Similarly to the uniform-cost case, for each variable $x \in \mathcal{X}$, the set $\mathcal{F}$ contains a nonnegative, real-valued function $\varphi_{x}$ with the scope

$$
Q_{x}= \begin{cases}\left\{x_{v}\right\}, & x=x_{v}, k=0 \\ \left\{x_{v}, x_{v}^{w_{k}}\right\}, & x=x_{v}, k>0 \\ \left\{x_{v}^{w_{1}}, x_{w_{1}}\right\}, & x=x_{v}^{w_{1}}, k>0 \\ \left\{x_{v}^{w_{j}}, x_{v}^{w_{j-1}}, x_{w_{j}}\right\}, & x=x_{v}^{w_{j}}, 1<j \leq k\end{cases}
$$

where $\operatorname{pred}(v)=\left\{w_{1}, \ldots, w_{k}\right\}$ (with $k=0$ meaning $\operatorname{pred}(v)=\emptyset$ ).

Proceeding now with specifying the functional components $\mathcal{F}$ of $\mathrm{COP}_{\Pi}$, first, for each $x_{v}$ with $\operatorname{pred}(v)=\emptyset$, and for each $\sigma_{v} \in \unrhd^{*}[v]$, we set $\varphi_{x_{v}}\left(\sigma_{v}\right)$ according to Eq. 22.

$$
\varphi_{x_{v}}\left(\sigma_{v}\right)= \begin{cases}0, & \left|\sigma_{v}\right|=1, \\ \mathcal{C}\left(a_{\mathrm{w}_{v}}\right), & \left|\sigma_{v}\right|=2, a_{\mathrm{w}_{v}} \in A_{v}, \\ \left\lceil\frac{\left|\sigma_{v}\right|-1}{2}\right\rceil \cdot \mathcal{C}\left(a_{\mathrm{w}_{v}}\right)+\left\lfloor\frac{\left|\sigma_{v}\right|-1}{2}\right\rfloor \cdot \mathcal{C}\left(a_{\mathrm{b}_{v}}\right), & \left|\sigma_{v}\right|>2, a_{\mathrm{w}_{v}}, a_{\mathrm{b}_{v}} \in A_{v}, \\ \infty, & \text { otherwise }\end{cases}
$$

In turn, for each planning variable $v \in V$ with $\operatorname{pred}(v)=\left\{w_{1}, \ldots, w_{k}\right\}, k>0$, the function $\varphi_{x_{v}}$ is set as in Eq. 23.

$$
\varphi_{x_{v}}\left(\sigma_{v},\left[\mathbf{S}, \#_{\mathrm{w}}, \#_{\mathbf{b}}, \eta\right]\right)= \begin{cases}0, & \left|\sigma_{v}\right|=1,\left[\mathbf{S}, \#_{\mathrm{w}}, \#_{\mathbf{b}}, \eta\right]=[\mathrm{S} 8,0,0,0], \\ 0, & \left|\sigma_{v}\right|>1,\left[\mathbf{S}, \#_{\mathrm{w}}, \#_{\mathbf{b}}, \eta\right]=\llbracket \mathrm{S} 1,\left\lceil\frac{\left|\sigma_{v}\right|-1}{2}\right\rceil,\left\lfloor\frac{\left|\sigma_{v}\right|-1}{2}\right\rfloor,\left|\sigma_{v}\right|-1 \rrbracket, \\ \infty, & \text { otherwise }\end{cases}
$$

The semantics of Eq. 23 is simple - if no value changes of $v$ are required, then trivially no $\operatorname{support}$ of $\operatorname{pred}(v)$ to $v$ is needed; otherwise, all possible roles for $\operatorname{pred}(v)$ should be considered.

Now, we proceed with specifying a generic function $\varphi$ that, for each $v \in V$, each $w \in \operatorname{pred}(v)$, and each $\left(\mathrm{R},\left[\mathbf{S}, \#_{\mathrm{w}}, \#_{\mathbf{b}}, \eta\right], \sigma_{w}\right) \in\{\mathrm{R} 1, \ldots, \mathrm{R} 10\} \times \operatorname{Dom}\left(x_{v}^{w}\right) \times \operatorname{Dom}\left(x_{w}\right)$, provides the marginal over the actions $A_{v}$ cost of $w$ taking the role $\mathrm{R}$, and under this role, supporting $\#_{\mathrm{w}}$ changes of $v$ to $\mathrm{w}_{v}$ and $\#_{\mathbf{b}}$ changes of $v$ to $\mathbf{b}_{v}$, out of total $\eta$ changes of $v$ needed. For ease of presentation, let $\xi\left(x_{1}, x_{2}, y_{1}, y_{2}\right)$ denote the cost of an action sequence consisting of $x_{1}$ actions of type $a_{\mathrm{w}_{v} \mid \mathrm{b}_{w}}, x_{2}$ actions of type $a_{\mathrm{w}_{v} \mid \mathrm{w}_{w}}, y_{1}$ actions of type $a_{\mathrm{b}_{v} \mid \mathrm{w}_{w}}$, $y_{2}$ actions of type $a_{\mathbf{b}_{v} \mid \mathbf{b}_{w}}$, that is

$$
\xi\left(x_{1}, x_{2}, y_{1}, y_{2}\right)=x_{1} \cdot \mathcal{C}\left(a_{\mathrm{w}_{v} \mid \mathbf{b}_{w}}\right)+x_{2} \cdot \mathcal{C}\left(a_{\mathrm{w}_{v} \mid \mathrm{w}_{w}}\right)+y_{1} \cdot \mathcal{C}\left(a_{\mathbf{b}_{v} \mid \mathrm{w}_{w}}\right)+y_{2} \cdot \mathcal{C}\left(a_{\mathbf{b}_{v} \mid \mathbf{b}_{w}}\right)
$$

While the notation $\xi_{v, w}$ is probably more appropriate for the semantics of $\xi$, we adopt the latter for its shortness because the identity of $v$ and $w$ will always be clear from the context.

The Eqs. 25-34 below specify $\varphi\left(\mathrm{R},\left[\mathbf{S}, \#_{\mathrm{w}}, \#_{\mathrm{b}}, \eta\right], \sigma_{w}\right)$ for $\mathrm{R} \in\{\mathrm{R} 1, \ldots, \mathrm{R} 10\}$. The semantics of $\varphi\left(\mathrm{R},\left[\mathbf{S}, \#_{\mathrm{w}}, \#_{\mathbf{b}}, \eta\right], \sigma_{w}\right)$ is to capture the minimal cumulative cost over the actions from $A_{v}$ to achieve $\#_{\mathrm{w}}$ and $\#_{\mathrm{b}}$ (out of $\eta$ ) value changes of $v$ under the support of the 
parent $w$ playing the role $\mathrm{R}$ with respect to $v$. For example, role $\mathrm{R} 3$ means supporting all the actions that change the value of $v$ to $\mathrm{w}_{v}$, and Eq. 27 gives us the minimal cost of this support in terms of the cumulative cost of the supported actions from $A_{v}$. These minimal costs are taken from the relevant cases in the proof of Theorem 8 , notably

(Eq. 25) Case $(I)$.

(Eq. 26) Cases $\{(I I I),(I V)\} \cdot\{2,4,6,8\}$.

(Eq. 27) Case $(I I)$, the cost of all actions that change the value of $v$ to $\mathrm{w}_{v}$.

(Eq. 28) Case $(I I)$, the cost of all actions that change the value of $v$ to $\mathrm{b}_{v}$.

(Eq. 29) Cases $\{(I I I),(I V)\} .\{1,3,5,7\} . a$, the minimal cost.

(Eq. 30) Cases $\{(I I I),(I V)\} .\{1,3,5,7\} . b$, the minimal cost.

(Eq. 31) Cases $\{(I I I),(I V)\} .\{1,3,5,7\} . a$, the cost of all actions prevailed by one parent.

(Eq. 32) Cases $\{(I I I),(I V)\} \cdot\{1,3,5,7\} . b$, the cost of all actions prevailed by one parent.

(Eq. 33) The residue of cases $\{(I I I),(I V)\} .\{1,3,5,7\} . a$. (Together with Eq. 31 this gives us the full cost of changing $v$ as required.)

(Eq. 34) The residue of cases $\{(I I I),(I V)\} .\{1,3,5,7\} . b$. (Together with Eq. 31 this gives us the full cost of changing $v$ as required.)

$$
\begin{aligned}
& \varphi\left(\mathrm{R} 1,\left[\mathbf{S}, \#_{\mathrm{w}}, \#_{\mathrm{b}}, \eta\right], \sigma_{w}\right)= \begin{cases}\xi\left(\#_{\mathrm{w}}, 0,0, \#_{\mathrm{b}}\right), & \left|\sigma_{w}\right|=1, \#_{\mathrm{w}}=\left\lceil\frac{\eta}{2}\right\rceil, \#_{\mathrm{b}}=\left\lfloor\frac{\eta}{2}\right\rfloor \\
\min \left\{\begin{array}{c}
\xi\left(\#_{\mathrm{w}}, 0,0, \#_{\mathrm{b}}\right), \\
\xi\left(0, \#_{\mathrm{w}}, \#_{\mathrm{b}}, 0\right)
\end{array}\right\}, & \left|\sigma_{w}\right|>1, \#_{\mathrm{w}}=\left\lceil\frac{\eta}{2}\right\rceil, \#_{\mathrm{b}}=\left\lfloor\frac{\eta}{2}\right\rfloor \\
\infty, & \text { otherwise }\end{cases} \\
& \varphi\left(\mathrm{R} 2,\left[\mathbf{S}, \#_{\mathrm{w}}, \#_{\mathbf{b}}, \eta\right], \sigma_{w}\right)= \begin{cases}\xi\left(\#_{\mathrm{w}}, 0, \#_{\mathrm{b}}, 0\right), & \left|\sigma_{w}\right|=\eta \geq 2, \#_{\mathrm{w}}=\left\lceil\frac{\eta}{2}\right\rceil, \#_{\mathbf{b}}=\left\lfloor\frac{\eta}{2}\right\rfloor \\
\min \left\{\begin{array}{c}
\xi\left(\#_{\mathrm{w}}, 0, \#_{\mathrm{b}}, 0\right), \\
\xi\left(0, \#_{\mathrm{w}}, 0, \#_{\mathbf{b}}\right)
\end{array}\right\}, & \left|\sigma_{w}\right|>\eta \geq 2, \#_{\mathrm{w}}=\left\lceil\frac{\eta}{2}\right\rceil, \#_{\mathbf{b}}=\left\lfloor\frac{\eta}{2}\right\rfloor \\
\infty, & \text { otherwise }\end{cases} \\
& \varphi\left(\mathrm{R} 3,\left[\mathbf{S}, \#_{\mathbf{w}}, \#_{\mathbf{b}}, \eta\right], \sigma_{w}\right)= \begin{cases}\#_{\mathrm{w}} \cdot \mathcal{C}\left(a_{\mathrm{w}_{v} \mid \mathbf{b}_{w}}\right), & \left|\sigma_{w}\right|=1, \#_{\mathrm{w}}=\left\lceil\frac{\eta}{2}\right\rceil, \#_{\mathbf{b}}=0 \\
\min \left\{\begin{array}{l}
\#_{\mathrm{w}} \cdot \mathcal{C}\left(a_{\mathrm{w}_{v} \mid \mathbf{b}_{w}}\right), \\
\#_{\mathrm{w}} \cdot \mathcal{C}\left(a_{\mathrm{w}_{v} \mid \mathrm{w}_{w}}\right)
\end{array}\right\}, & \left|\sigma_{w}\right|>1, \#_{\mathrm{w}}=\left\lceil\frac{\eta}{2}\right\rceil, \#_{\mathbf{b}}=0 \\
\infty, & \text { otherwise }\end{cases} \\
& \varphi\left(\mathrm{R} 4,\left[\mathbf{S}, \#_{\mathrm{w}}, \#_{\mathrm{b}}, \eta\right], \sigma_{w}\right)= \begin{cases}\#_{\mathrm{b}} \cdot \mathcal{C}\left(a_{\mathrm{b}_{v} \mid \mathbf{b}_{w}}\right), & \left|\sigma_{w}\right|=1, \#_{\mathrm{w}}=0, \#_{\mathrm{b}}=\left\lfloor\frac{\eta}{2}\right\rfloor \\
\min \left\{\begin{array}{l}
\#_{\mathrm{b}} \cdot \mathcal{C}\left(a_{\mathrm{b}_{v} \mid \mathrm{b}_{w}}\right), \\
\#_{\mathrm{b}} \cdot \mathcal{C}\left(a_{\mathrm{b}_{v} \mid \mathrm{w}_{w}}\right)
\end{array}\right\}, & \left|\sigma_{w}\right|>1, \#_{\mathrm{w}}=0, \#_{\mathbf{b}}=\left\lfloor\frac{\eta}{2}\right\rfloor \\
\infty, & \text { otherwise }\end{cases}
\end{aligned}
$$




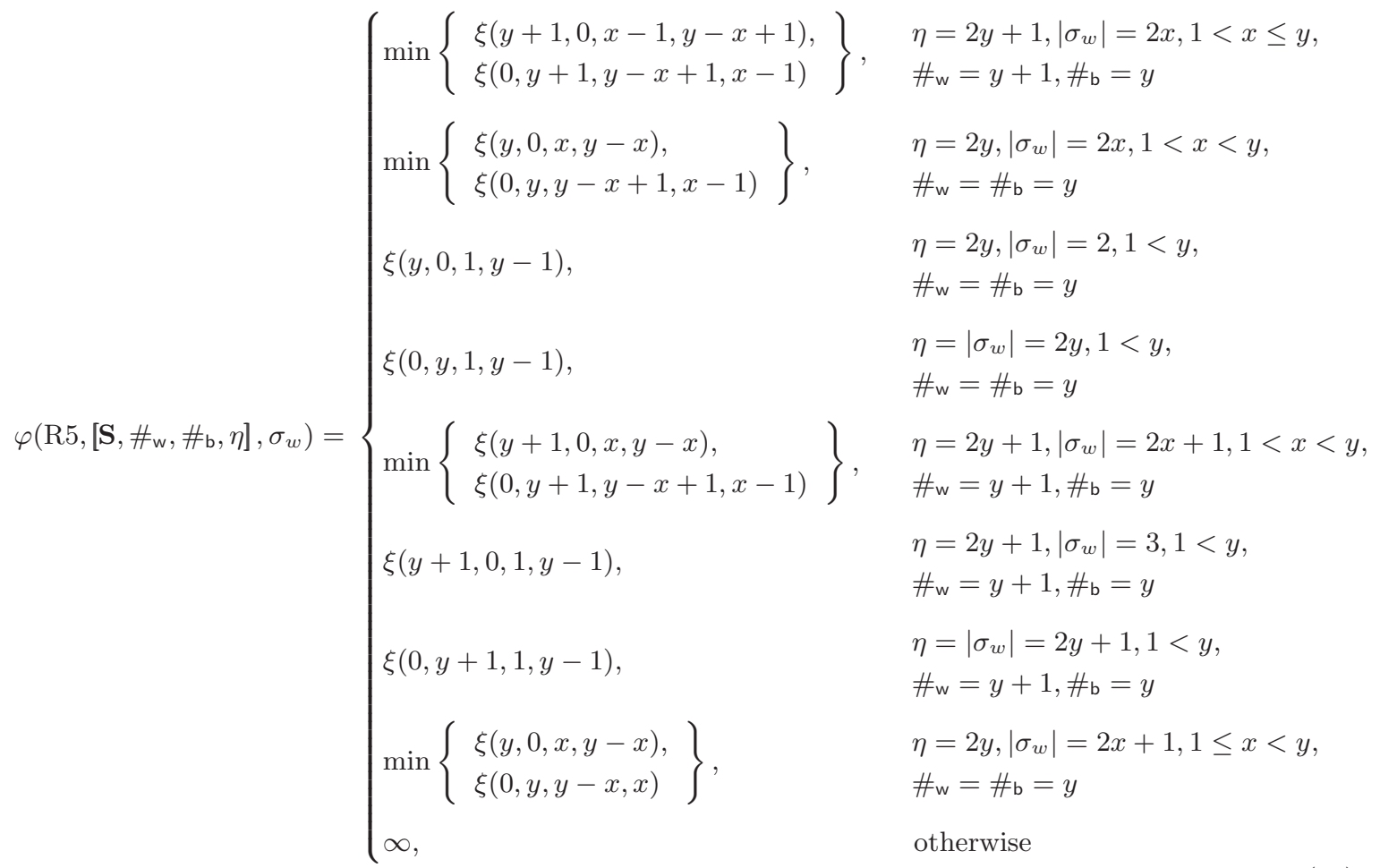

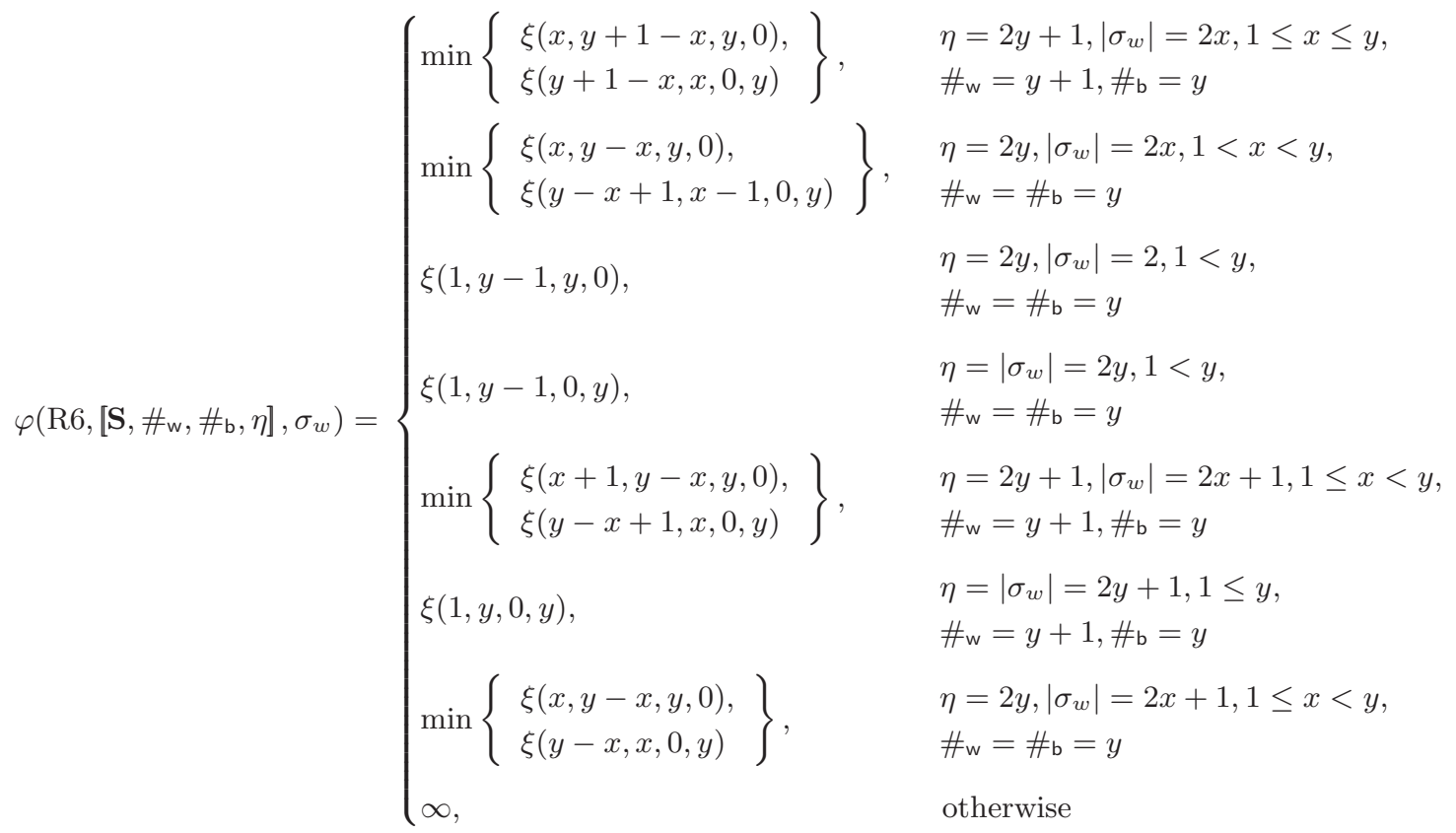


Tractable Cost-Optimal Planning

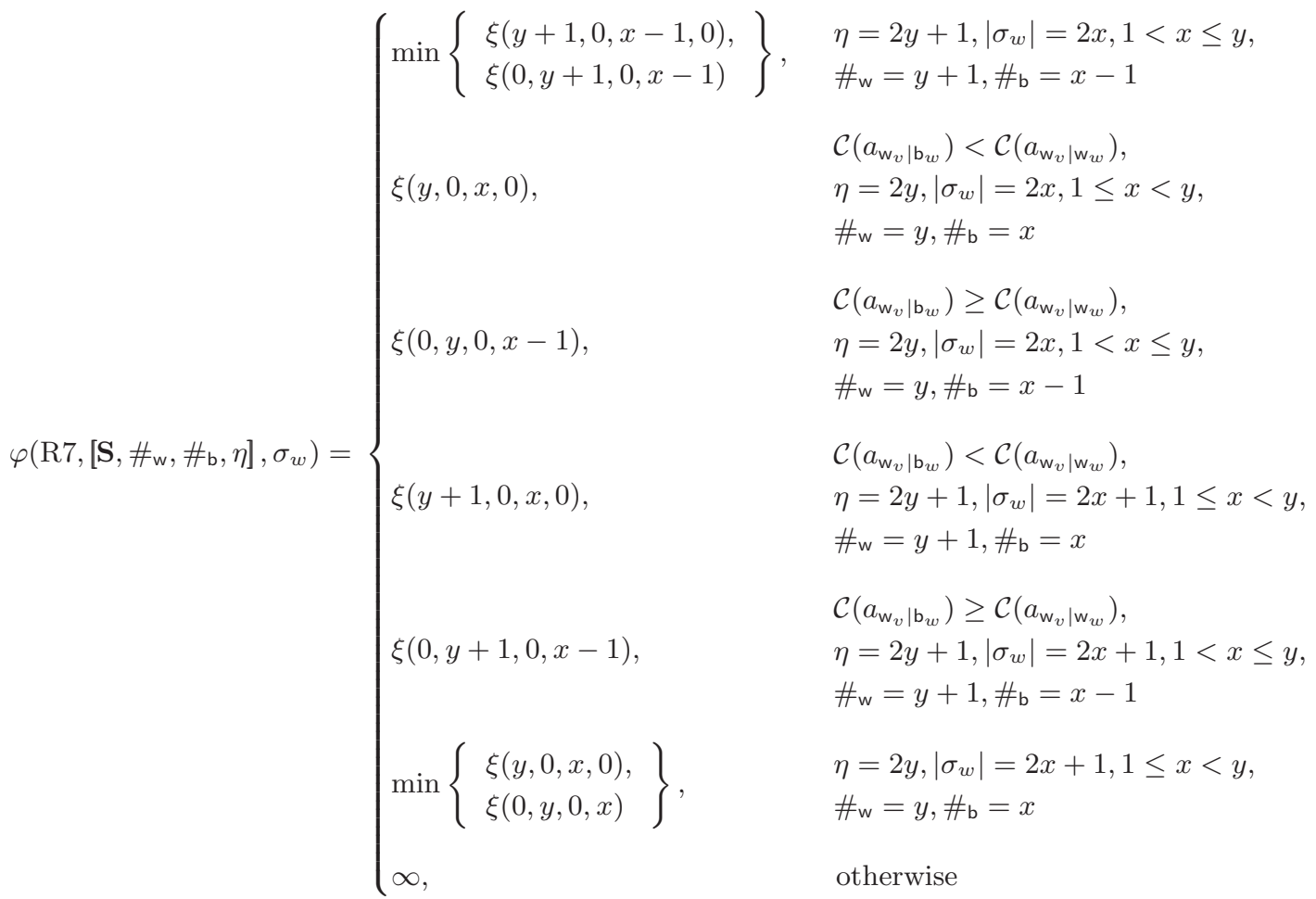

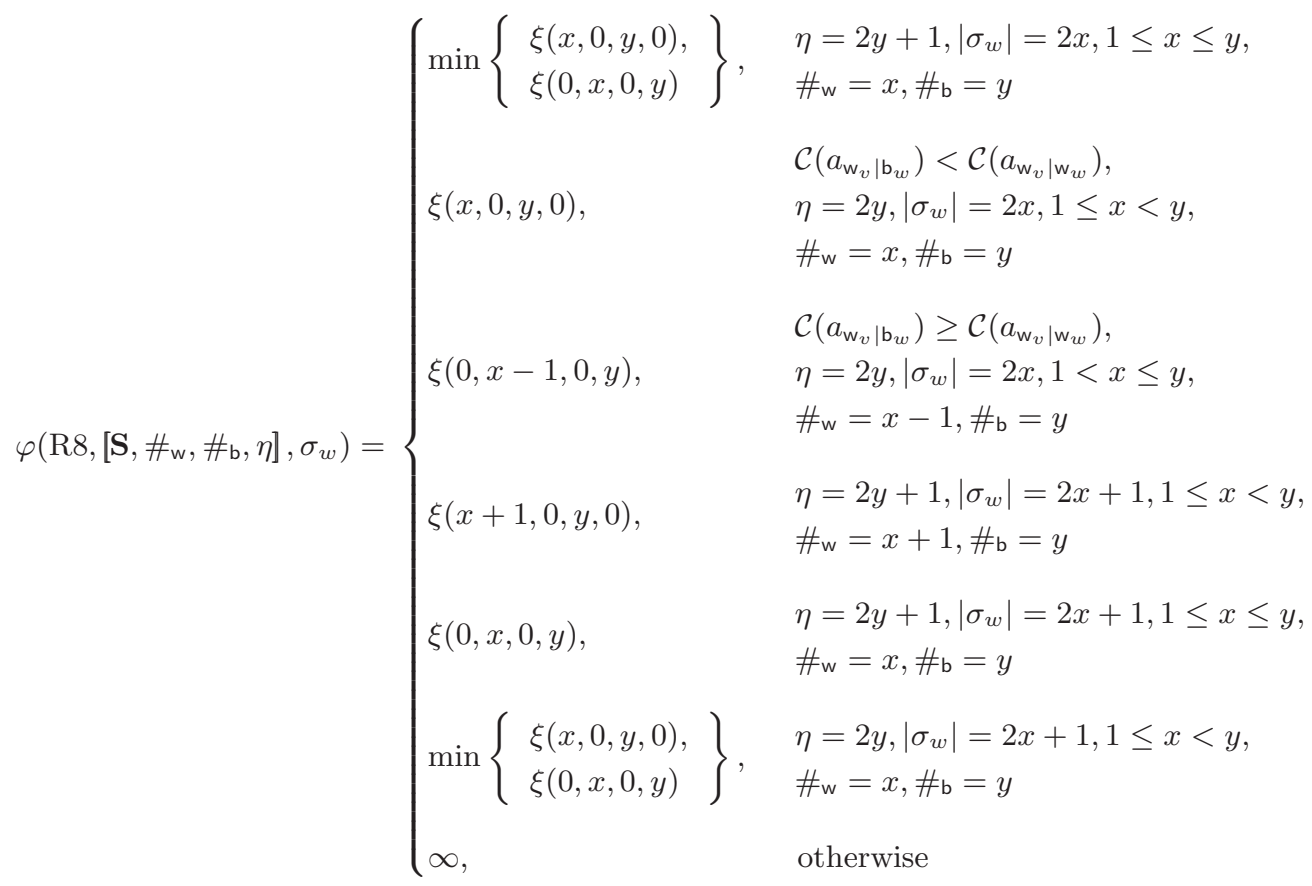




$$
\begin{gathered}
\varphi\left(\mathrm{R} 9,\left[\mathbf{S}, \#_{\mathrm{w}}, \#_{\mathrm{b}}, \eta\right], \sigma_{w}\right)= \begin{cases}\#_{\mathrm{b}} \cdot \mathcal{C}\left(a_{\mathrm{b}_{v} \mid \mathrm{w}_{w}}\right), & \left|\sigma_{w}\right|=1, \#_{\mathrm{w}}=0, \#_{\mathrm{b}}<\left\lfloor\frac{\eta}{2}\right\rfloor \\
\min \left\{\begin{array}{l}
\#_{\mathrm{b}} \cdot \mathcal{C}\left(a_{\mathrm{b}_{v} \mid \mathrm{w}_{w}}\right), \\
\#_{\mathrm{b}} \cdot \mathcal{C}\left(a_{\mathrm{b}_{v} \mid \mathrm{b}_{w}}\right)
\end{array}\right\}, & \left|\sigma_{w}\right|>1, \#_{\mathrm{w}}=0, \#_{\mathrm{b}}<\left\lfloor\frac{\eta}{2}\right\rfloor \\
\infty, & \text { otherwise }\end{cases} \\
\varphi\left(\mathrm{R} 10,\left[\mathbf{S}, \#_{\mathrm{w}}, \#_{\mathrm{b}}, \eta \rrbracket, \sigma_{w}\right)= \begin{cases}\#_{\mathrm{w}} \cdot \mathcal{C}\left(a_{\mathrm{w}_{v} \mid \mathrm{b}_{w}}\right), & \left|\sigma_{w}\right|=1, \#_{\mathrm{w}}<\left\lceil\frac{\eta}{2}\right\rceil, \#_{\mathrm{b}}=0 \\
\min \left\{\begin{array}{l}
\#_{\mathrm{w}} \cdot \mathcal{C}\left(a_{\mathrm{w}_{v} \mid \mathrm{b}_{w}}\right), \\
\#_{\mathrm{w}} \cdot \mathcal{C}\left(a_{\mathrm{w}_{v} \mid \mathrm{w}_{w}}\right)
\end{array}\right\}, & \left|\sigma_{w}\right|>1, \#_{\mathrm{w}}<\left\lceil\frac{\eta}{2}\right\rceil, \#_{\mathrm{b}}=0 \\
\infty, & \text { otherwise }\end{cases} \right.
\end{gathered}
$$

Having specified the function $\varphi$, we now use it, in particular, for specifying the functional component $\varphi_{x_{v}}^{w_{1}}$ as in Eq. 35. This equation actually emulates movements in the state machine for $v$ as in Figure 12 to the terminal state $S 8$.

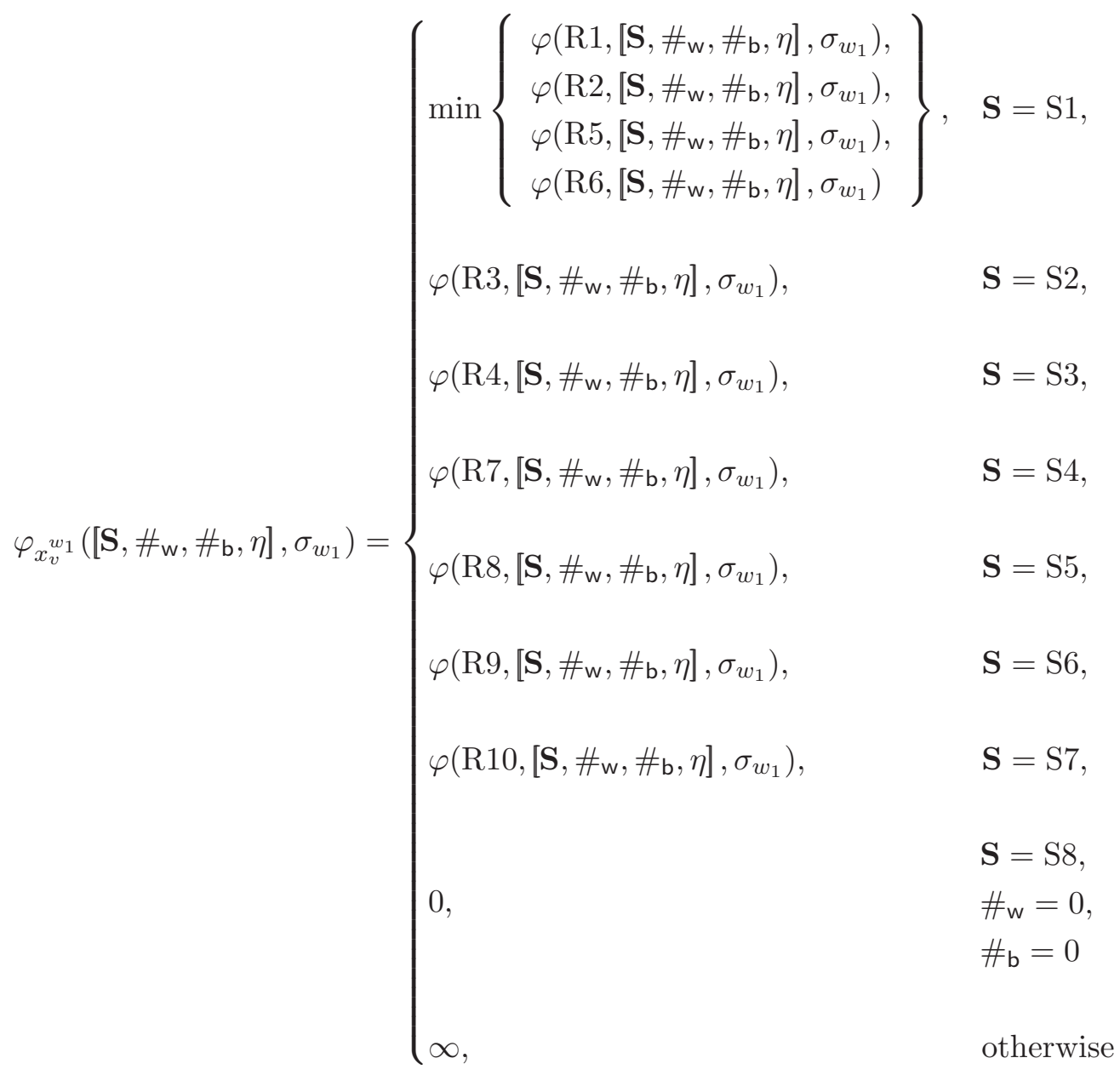

We now proceed with the rest of the functional components $\varphi_{x_{v}}^{w_{2}}, \ldots, \varphi_{x_{v}}^{w_{k}}$. For each $2 \leq j \leq k$, each $\left[\mathbf{S}, \#_{\mathrm{w}}, \#_{\mathbf{b}}, \eta\right] \in \operatorname{Dom}\left(x_{v}^{w_{j}}\right)$, each $\left[\mathbf{S}^{\prime}, \#_{\mathrm{w}}^{\prime}, \#_{\mathbf{b}}^{\prime}, \eta^{\prime}\right] \in \operatorname{Dom}\left(x_{v}^{w_{j-1}}\right)$, and each $\sigma_{w} \in \operatorname{Dom}\left(x_{w_{j}}\right)=\unrhd^{*}\left[w_{j}\right]$, the value of $\varphi_{x_{v}}^{w_{j}}$ is set according to Eq. 36. This equation also 
emulates movements in the state machine for $v$ as in Figure 12-each sub-case of Eq. 36 deals with a certain transition in that state machine.

$$
\begin{aligned}
& \varphi_{x_{v} w_{j}}\left(\left[\mathbf{S}, \#_{\mathbf{w}}, \# \mathbf{b}, \eta\right], \llbracket \mathbf{S}^{\prime}, \#_{\mathrm{w}}^{\prime}, \#_{\mathrm{b}}^{\prime}, \eta^{\prime} \rrbracket, \sigma_{w_{j}}\right)= \\
& \min \left\{\begin{array}{l}
\varphi\left(\mathrm{R} 1,\left[\mathbf{S}, \#_{\mathrm{w}}-\#_{\mathrm{w}}^{\prime}, \#_{\mathrm{b}}-\#_{\mathrm{b}}^{\prime}, \eta\right], \sigma_{w_{j}}\right), \\
\varphi\left(\mathrm{R} 2,\left[\mathbf{S}, \#_{\mathrm{w}}-\#_{\mathrm{w}}^{\prime}, \#_{\mathrm{b}}-\#_{\mathrm{b}}^{\prime}, \eta\right], \sigma_{w_{j}}\right), \\
\varphi\left(\mathrm{R} 5,\left[\mathbf{S}, \#_{\mathrm{w}}-\#_{\mathrm{w}}^{\prime}, \#_{\mathrm{b}}-\#_{\mathrm{b}}^{\prime}, \eta\right], \sigma_{w_{j}}\right), \\
\varphi\left(\mathrm{R} 6,\left[\mathbf{S}, \#_{\mathrm{w}}-\#_{\mathrm{w}}^{\prime}, \#_{\mathrm{b}}-\#_{\mathrm{b}}^{\prime}, \eta\right], \sigma_{w_{j}}\right)
\end{array}\right\}, \quad \begin{array}{l}
\mathbf{S}=\mathrm{S} 1, \mathbf{S}^{\prime}=\mathrm{S} 8, \eta=\eta^{\prime}, \\
\#_{\mathrm{w}} \geq \#_{\mathrm{w}}^{\prime}, \#_{\mathrm{b}} \geq \#_{\mathrm{b}}^{\prime}
\end{array}
\end{aligned}
$$

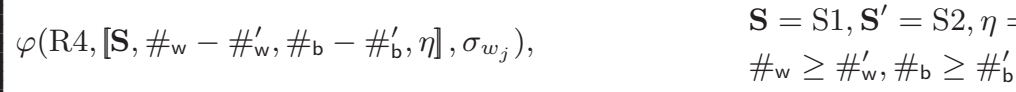

$$
\begin{aligned}
& \varphi\left(\mathrm{R} 3,\left[\mathbf{S}, \#_{\mathrm{w}}-\#_{\mathrm{w}}^{\prime}, \#_{\mathrm{b}}-\#_{\mathrm{b}}^{\prime}, \eta\right], \sigma_{w_{j}}\right), \quad \mathbf{S}=\mathrm{S} 1, \mathbf{S}^{\prime}=\mathrm{S} 3, \eta=\eta^{\prime}, \\
& \#_{\mathrm{w}} \geq \#_{\mathrm{w}}^{\prime}, \#_{\mathrm{b}} \geq \#_{\mathrm{b}}^{\prime} \\
& \varphi\left(\mathrm{R} 9,\left[\mathbf{S}, \#_{\mathrm{w}}-\#_{\mathrm{w}}^{\prime}, \#_{\mathrm{b}}-\#_{\mathrm{b}}^{\prime}, \eta\right], \sigma_{w_{j}}\right), \quad \mathbf{S}=\mathrm{S} 1, \mathbf{S}^{\prime}=\mathrm{S} 4, \eta=\eta^{\prime}, \\
& \#_{\mathrm{w}} \geq \#_{\mathrm{w}}^{\prime}, \#_{\mathrm{b}} \geq \#_{\mathrm{b}}^{\prime} \\
& \mathbf{S}=\mathrm{S} 1, \mathbf{S}^{\prime}=\mathrm{S} 5, \eta=\eta^{\prime}, \\
& \#_{\mathrm{w}} \geq \#_{\mathrm{w}}^{\prime}, \#_{\mathrm{b}} \geq \#_{\mathrm{b}}^{\prime} \\
& \mathbf{S}=\mathrm{S} 1, \mathbf{S}^{\prime}=\mathrm{S} 6, \eta=\eta^{\prime}, \\
& \#_{\mathrm{w}} \geq \#_{\mathrm{w}}^{\prime}, \#_{\mathrm{b}} \geq \#_{\mathrm{b}}^{\prime} \\
& \mathbf{S}=\mathrm{S} 1, \mathbf{S}^{\prime}=\mathrm{S} 7, \eta=\eta^{\prime}, \\
& \#_{\mathrm{w}} \geq \#_{\mathrm{w}}^{\prime}, \#_{\mathrm{b}} \geq \#_{\mathrm{b}}^{\prime} \\
& \mathbf{S}=\mathrm{S} 2, \mathbf{S}^{\prime}=\mathrm{S} 8, \eta=\eta^{\prime}, \\
& \varphi\left(\mathrm{R} 3,\left[\mathbf{S}, \#_{\mathrm{w}}-\#_{\mathrm{w}}^{\prime}, \#_{\mathrm{b}}-\#_{\mathrm{b}}^{\prime}, \eta\right], \sigma_{w_{j}}\right), \quad \quad \#_{\mathrm{w}} \geq \#_{\mathrm{w}}^{\prime}, \#_{\mathrm{b}} \geq \#_{\mathrm{b}}^{\prime} \\
& \varphi\left(\mathrm{R} 4,\left[\mathbf{S}, \#_{\mathrm{w}}-\#_{\mathrm{w}}^{\prime}, \#_{\mathrm{b}}-\#_{\mathrm{b}}^{\prime}, \eta\right], \sigma_{w_{j}}\right), \quad \mathbf{S}=\mathrm{S} 3, \mathbf{S}^{\prime}=\mathrm{S} 8, \eta=\eta^{\prime}, \\
& \#_{\mathrm{w}} \geq \#_{\mathrm{w}}^{\prime}, \#_{\mathrm{b}} \geq \#_{\mathrm{b}}^{\prime} \\
& \varphi\left(\mathrm{R} 7,\left[\mathbf{S}, \#_{\mathrm{w}}-\#_{\mathrm{w}}^{\prime}, \#_{\mathrm{b}}-\#_{\mathrm{b}}^{\prime}, \eta\right], \sigma_{w_{j}}\right), \quad \mathbf{S}=\mathrm{S} 4, \mathbf{S}^{\prime}=\mathrm{S} 8, \eta=\eta^{\prime}, \\
& \#_{\mathrm{w}} \geq \#_{\mathrm{w}}^{\prime}, \#_{\mathrm{b}} \geq \#_{\mathrm{b}}^{\prime} \\
& \mathbf{S}=\mathrm{S} 5, \mathbf{S}^{\prime}=\mathrm{S} 8, \eta=\eta^{\prime}, \\
& \varphi\left(\mathrm{R} 8,\left[\mathbf{S}, \#_{\mathrm{w}}-\#_{\mathrm{w}}^{\prime}, \#_{\mathrm{b}}-\#_{\mathrm{b}}^{\prime}, \eta\right], \sigma_{w_{j}}\right), \quad \#_{\mathrm{w}} \geq \#_{\mathrm{w}}^{\prime}, \#_{\mathrm{b}} \geq \#_{\mathrm{b}}^{\prime} \\
& \varphi\left(\mathrm{R} 9,\left[\mathbf{S}, \#_{\mathrm{w}}-\#_{\mathrm{w}}^{\prime}, \#_{\mathrm{b}}-\#_{\mathrm{b}}^{\prime}, \eta\right], \sigma_{w_{j}}\right), \quad \mathbf{S}=\mathrm{S} 6, \mathbf{S}^{\prime}=\mathrm{S} 8, \eta=\eta^{\prime}, \\
& \#_{\mathrm{w}} \geq \#_{\mathrm{w}}^{\prime}, \#_{\mathrm{b}} \geq \#_{\mathrm{b}}^{\prime} \\
& \mathbf{S}=\mathrm{S} 7, \mathbf{S}^{\prime}=\mathrm{S} 8, \eta=\eta^{\prime}, \\
& \#_{\mathrm{w}} \geq \#_{\mathrm{w}}^{\prime}, \#_{\mathrm{b}} \geq \#_{\mathrm{b}}^{\prime} \\
& \mathbf{S}=\mathbf{S}^{\prime}, \eta=\eta^{\prime}, \\
& \#_{\mathrm{w}}=\#_{\mathrm{w}}^{\prime}, \#_{\mathrm{b}}=\#_{\mathrm{b}}^{\prime} \\
& \text { otherwise }
\end{aligned}
$$

This finalizes the construction of $\mathrm{COP}_{\Pi}$, and this construction constitutes the first three steps of the algorithm polytree-1-dep in Figure 13(a). The subsequent steps of this algorithm are conceptually similar to these of the polytree-1-dep-uniform algorithm in Section 4. It is 


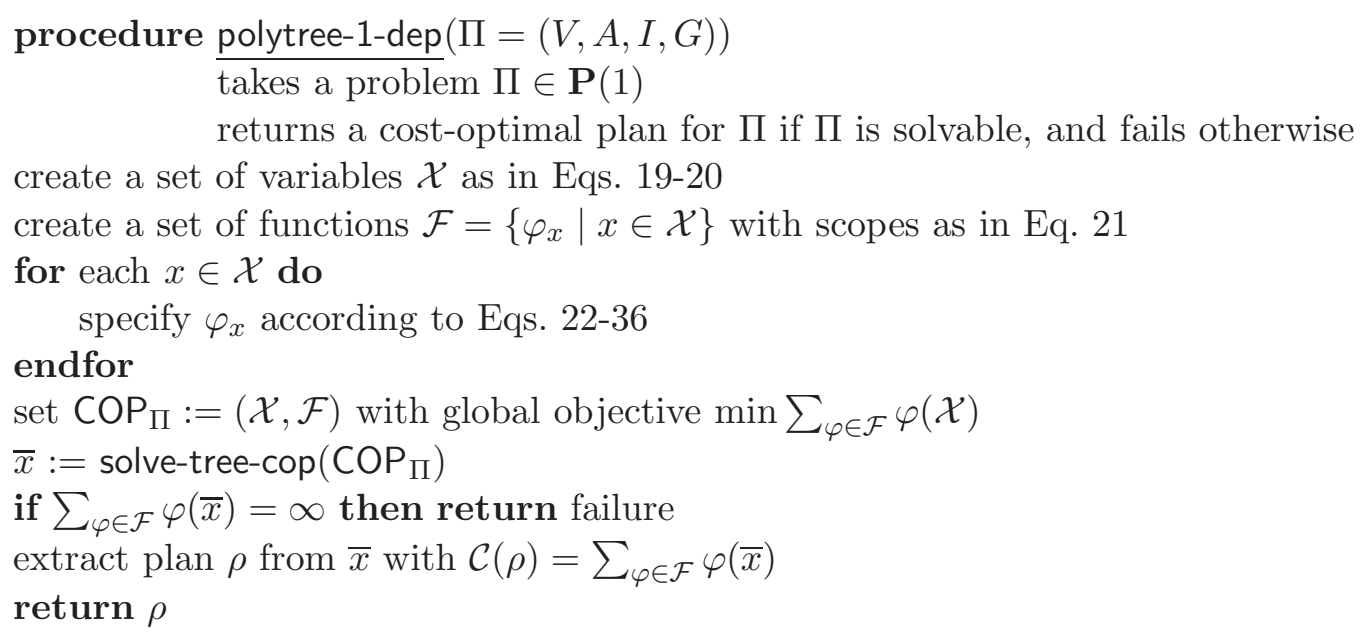

Figure 13: Algorithm for cost-optimal planning for $\mathbf{P}(1)$ problems.

not hard to verify from Eqs. 19-21, and the fact that the causal graph of $\Pi \in \mathbf{P}(1)$ forms a polytree that

(i) for each variable $x \in \mathcal{X},|\operatorname{Dom}(x)|=\operatorname{poly}(n)$,

(ii) the tree-width of the cost network of $\mathcal{F}$ is $\leq 3$, and

(iii) the optimal tree-decomposition of the $\mathrm{COP}_{\Pi}$ 's cost network is given by any topological ordering of the causal graph that is consistent with the (arbitrary yet fixed at the time of the $\mathrm{COP}_{\Pi}$ 's construction) orderings of each planning variable's parents in the causal graph.

Theorem 9 Let $\Pi$ be a $\mathbf{P}(1)$ problem, $\operatorname{COP}_{\Pi}=(\mathcal{X}, \mathcal{F})$ be the corresponding constraint optimization problem, and $\bar{x}$ be an optimal assignment to $\mathcal{X}$ with $\sum_{\varphi \in \mathcal{F}} \varphi(\bar{x})=\alpha$.

(I) If $\alpha<\infty$, then a plan of cost $\alpha$ for $\Pi$ can be reconstructed from $\bar{x}$ in time polynomial in the description size of $\Pi$.

(II) If $\Pi$ has a plan, then $\alpha<\infty$.

Proof Sketch: The proof of Theorem 9 is in Appendix A, pp. 278-286; here we provide only a sketch of the proof's skeleton. To prove (I), given a COP solution $\bar{x}=\left\{\sigma_{v_{1}}, \ldots, \sigma_{v_{n}}\right\}$ with $\sum_{\varphi \in \mathcal{F}} \varphi(\bar{x})=\alpha<\infty$, we construct a plan $\rho$ for $\Pi$ with $\mathcal{C}(\rho)=\alpha$. This is done by constructing action sequences $\rho_{v}$ for each $v \in V$, as well as constructing partial orders over the elements of these sequences of each variable and its parents. This construction distinguishes between numerous possibilities of the (joint) role that can be taken by the parents of the variable in question. The constructed orders over the "local" actions sequences are then combined and linearized (using Theorem 1) into an action sequence $\rho$ that is a valid plan for $\Pi$ with $\mathcal{C}(\rho)=\sum_{v \in V} \mathcal{C}\left(\rho_{v}\right)=\sum_{v \in V} \varphi_{v}(\bar{x})=\sum_{\varphi \in \mathcal{F}} \varphi(\bar{x})=\alpha$. To prove (II), given solvable problem $\Pi$ and some irreducible post-3/2 plan $\rho$ for $\Pi$, we construct a COP assignment $\bar{x}_{\rho}$ such that $\sum_{\varphi \in \mathcal{F}} \varphi\left(\bar{x}_{\rho}\right)=\mathcal{C}(\rho)$. Then, from $\alpha \leq \sum_{\varphi \in \mathcal{F}} \varphi\left(\bar{x}_{\rho}\right)$ and $\mathcal{C}(\rho)<\infty$, we obtain the claimed $\alpha<\infty$. 
Theorem 10 Cost-optimal planning for $\mathbf{P}(1)$ is tractable.

Proof: Given a planning problem $\Pi \in \mathbf{P}(1)$, we show that the corresponding constraint optimization problem $\mathrm{COP}_{\Pi}$ can be constructed and solved in time polynomial in the description size of $\Pi$. Let $n$ be the number of state variables in $\Pi$. In polytree-1-dep, we first construct the constraint optimization problem $\operatorname{COP}_{\Pi}$ over $\Theta\left(n^{2}\right)$ variables $\mathcal{X}$ with domain sizes being bounded either by $O(n)$ or by $O\left(n^{3}\right)$ (for COP variables representing state variables and causal graph edges, respectively). The number of functional components in $\mathrm{COP}_{\Pi}$ is $\Theta\left(n^{2}\right)$, each defined over one variable with domain size of $O(n)$ and either one or two variables with domain sizes of $O\left(n^{3}\right)$. The construction is linear in the size of the resulting $\mathrm{COP}$, and thus is accomplished in time $O\left(n^{9}\right)$.

Applying then to $\mathrm{COP}_{\Pi}$ a tree-decomposition that clusters the scopes of the functional components $\mathcal{F}$, we arrive into an equivalent, tree-structured constraint optimization problem over $\Theta\left(n^{2}\right)$ variables with domains of size $O\left(n^{7}\right)$. Such a tree-structured COP can be solved

in time $O\left(x y^{2}\right)$ where $x$ is the number of variables and $y$ is an upper bound on the size of a variable's domain (Dechter, 2003). Therefore, solving $\mathrm{COP}_{\Pi}$ can be done in time $O\left(n^{16}\right)$. As this dominates both the time complexity of constructing $\mathrm{COP}_{\Pi}$, and the time complexity of extracting a plan from the optimal solution to $\mathrm{COP}_{\Pi}$ (see the proof of (I) in Theorem 9), the overall complexity of the algorithm polytree-1-dep is $O\left(n^{16}\right)$, and therefore polynomial in the description size of $\Pi$.

\section{Drawing the Limits of $k$-Dependence}

Having read this far, the reader may wonder whether 1-dependence is not a strong enough property to make the cost-planning tractable even for some more complex than polytree forms of the causal graph. In this last technical section we discuss the limits of the power of $k$-dependence (and, in particular, of 1-dependence), and present some negative results that draw a boundary between the tractable and intractable $k$-dependent UB problems.

- In Theorem 11 we show that cost-optimal planning is already hard for the $\mathbf{S}_{b}^{b}(1)$ problem class, that is, the class of 1-dependent UB problems inducing directed-path singly connected causal graphs with both in- and out-degrees being bounded by a constant. This result further stresses the connection between the undirected cycles in the causal graph and the complexity of various planning tasks, which has been first discussed by Brafman and Domshlak (2003).

- In Theorem 12 we show that even non-optimal planning is hard for the $\mathbf{S}_{b}^{b}(2)$ problem class. This results suggests that 1 -dependence is a rather special case of $k$-dependence in terms of the connection to computational tractability. However, given the (still) empty entries in Figures 5a and 5b, further analysis of the "criticality" of 1-dependence is needed.

Theorem 11 Cost-optimal planning for $\mathbf{S}_{b}^{b}(1)$ is NP-complete.

Proof: The membership in NP is implied by Theorem 2 by Brafman and Domshlak (2003). The proof of NP-hardness is by a polynomial reduction from the well-known Vertex Cover problem (Garey \& Johnson, 1978). The problem of Vertex Cover is: given an undirected 
graph $\mathbf{G}=(\mathbf{V}, \mathbf{E})$, find a minimal-size subset $\mathbf{V}^{\prime}$ of $\mathbf{V}$ such that each edge in $\mathbf{E}$ has at least one of its two end-nodes in $\mathbf{V}^{\prime}$. Given an undirected graph $\mathbf{G}=(\mathbf{V}, \mathbf{E})$, let the planning problem $\Pi_{\mathbf{G}}=\left\langle V_{\mathbf{G}}, A_{\mathbf{G}}, I_{\mathbf{G}}, G_{\mathbf{G}}\right\rangle$ be defined as follows.

- $V_{\mathbf{G}}=\left\{v_{1}, \ldots, v_{|\mathbf{V}|}, u_{1}, \ldots, u_{|\mathbf{E}|}\right\}$, and, for all $v_{i}, u_{j}, \operatorname{Dom}\left(v_{i}\right)=\operatorname{Dom}\left(u_{j}\right)=\{T, F\}$,

- $I_{\mathbf{G}}=\left\{v_{i}=F \mid v_{i} \in V_{\mathbf{G}}\right\} \bigcup\left\{u_{i}=F \mid u_{i} \in V_{\mathbf{G}}\right\}$,

- $G_{\mathbf{G}}=\left\{u_{i}=T \mid u_{i} \in V_{\mathbf{G}}\right\}$,

- Actions $A_{\mathbf{G}}=A_{\mathbf{V}} \cup A_{\mathbf{E}}$, where $A_{\mathbf{V}}=\left\{a_{v_{1}}, \ldots, a_{v_{|\mathbf{V}|}}\right\}$ with

$$
\operatorname{pre}\left(a_{v_{i}}\right)=\left\{v_{i}=F\right\}, \quad \operatorname{eff}\left(a_{v_{i}}\right)=\left\{v_{i}=T\right\}, \mathcal{C}\left(a_{v_{i}}\right)=1
$$

and $A_{\mathbf{E}}=\left\{a_{u_{1}}, a_{u_{1}}^{\prime}, \ldots, a_{u_{|\mathbf{E}|}}, a_{u_{|\mathbf{E}|}}^{\prime}\right\}$ with

$$
\begin{aligned}
\operatorname{pre}\left(a_{u_{i}}\right) & =\left\{u_{i}=F, v_{i_{1}}=T\right\}, \\
\operatorname{pre}\left(a_{u_{i}}^{\prime}\right) & =\left\{u_{i}=F, v_{i_{2}}=T\right\}, \\
\operatorname{eff}\left(a_{u_{i}}\right)=\operatorname{eff}\left(a_{u_{i}}^{\prime}\right) & =\left\{u_{i}=T\right\}, \\
\mathcal{C}\left(a_{u_{i}}\right)=\mathcal{C}\left(a_{u_{i}}^{\prime}\right) & =0
\end{aligned}
$$

where the variables $v_{i_{1}}, v_{i_{2}}$ correspond to the endpoints of the edge corresponding to the variable $u_{i}$.

Given this construction of $\Pi_{\mathbf{G}}$, it is easy to see that (i) any plan $\rho$ for $\Pi_{\mathbf{G}}$ provides us with a vertex cover $\mathbf{V}_{\rho}$ for $\mathbf{G}$ such that $\left|\mathbf{V}_{\rho}\right|=\mathcal{C}(\rho)$ and vice versa, and thus (ii) costoptimal plans for $\Pi_{\mathbf{G}}$ (and only such plans for $\Pi_{\mathbf{G}}$ ) provide us with minimal vertex covers for $\mathbf{G}$. The topology of the causal graph of $\Pi_{\mathbf{G}}$ is as required, and 1-dependence of $\Pi_{\mathbf{G}}$ is immediate from Eqs. 37-38. This finalizes the proof of NP-completeness of cost-optimal planning for $\mathbf{S}_{b}^{b}(1)$.

Theorem 12 Planning for $\mathbf{S}_{b}^{b}(2)$ is NP-complete.

Proof: The proof is basically given by the construction in the proof by Brafman and Domshlak (2003) for their Theorem 2. The polynomial reduction there is from 3-SAT to planning for S. Observing that 3-SAT remains hard even if no variable participates in more than five clauses of the formula (Garey \& Johnson, 1978), and that the reduction of Brafman and Domshlak from such 3-SAT formulas is effectively to planning for $\mathbf{S}_{b}^{b}(2)$, accomplishes the proof of our claim.

\section{Conclusion and Future Work}

One of the key conclusions by Bylander (1994) in his seminal article on planning complexity was that "... the analysis strongly suggests that there is no such thing as a set of generallyapplicable domain independent properties that lead to efficient planning." The later works by, e.g., Bäckström and Nebel (1995), Jonsson and Bäckström (1998a), and Brafman and Domshlak $(2003,2006)$ have shown that this conclusion was too pessimistic. By considering not only local restrictions on actions, but also global restrictions on the action sets, as well 
as some structural properties of the problems, these works managed to identify numerous domain-independent tractable fragments of classical planning. Having said that, the palette of known tractable fragments of planning remains limited, and even less is known about tractable optimal planning. While there is no difference in theoretical complexity of regular and optimal planning in the general case (Bylander, 1994), many of the classical planning domains are provably easy to solve, but hard to solve optimally (Helmert, 2003).

In this work we have studied the complexity of cost-optimal classical planning over propositional state variables and unary-effect actions. We discovered novel problem fragments for which such optimization is tractable, and identified certain conditions that differentiate between tractable and intractable problems. The results are based on exploiting certain structural and syntactic characteristics of planning problems. Almost all our tractability results are based on a proof technique that connects between certain tools from planning and tractable constraint optimization, and we believe that this technique is in itself interesting due to a clear evidence for its robustness - our different algorithms exploit this proof technique, but in very much different manners.

Our results suggest that discovering new islands of tractability of optimal planning is not hopeless, and we strongly believe that this is indeed the case. In particular, our ongoing work is devoted both to the questions that have been left open in this paper (see Figure 5), as well as to planning problems with simple causal graphs but with multi-valued (in contrast to propositional) state variables. In fact, recently we have reported some preliminary positive results in the latter direction (Katz \& Domshlak, 2007). Interestingly, these recent results have been presented in a context of a potential "customer" for such tractability results, namely, in the context of homomorphism abstractions for admissible heuristics for general planning as heuristic search.

\section{Acknowledgments}

This research is supported in part by the Israel Science Foundations grants 2008100 and 2009589, as well as by the C. Wellner Research Fund. We thank Adele Howe and the anonymous reviewers whose attentive comments and helpful suggestions have greatly improved this paper.

\section{Appendix A. Proofs}

Theorem 1 Let $G$ be a polytree over vertices $V=\{1, \ldots, n\}$, and $\operatorname{pred}(i) \subset V$ denote the immediate predecessors of $i$ in $G$. For each $i \in V$, let $O_{i}$ be a finite set of objects associated with the vertex $i$, with the sets $O_{1}, \ldots, O_{n}$ being pairwise disjoint. For each $i \in V$, let $>_{i}$ be a strict partial order over $O_{i}$, and, for each $j \in \operatorname{pred}(i)$, let $>_{i, j}$ be a strict partial order over $O_{i} \cup O_{j}$.

If, for each $i \in V, j \in \operatorname{pred}(i)$, the transitively closed $>_{i} \cup>_{i, j}$ and $>_{j} \cup>_{i, j}$ induce (strict) partial orders over $O_{i} \cup O_{j}$, then so does the transitively closed

$$
>=\bigcup_{i \in V}\left(>_{i} \cup \bigcup_{j \in \operatorname{pred}(i)}>_{i, j}\right)
$$


over $O=\bigcup_{i \in V} O_{i}$.

Proof: In what follows, by $o_{i}$ we denote an arbitrary object from $O_{i}$. Assume to the contrary that all $>_{i} \cup>_{i, j}$ and $>_{j} \cup>_{i, j}$ are (strict) partial orders, and yet $>$ is not so. That is, there exists a pair of objects $o_{i}, o_{j} \in O$ for which hold both $o_{i}>o_{j}$ and $o_{j}>o_{i}$. By the construction of $>$, we have that there is a, possibly empty, path between the vertices $i$ and $j$ in the undirected graph induced by $G$. Since $G$ is a polytree, we know that such an undirected path

$$
i=i_{0} \cdot i_{1} \cdot \ldots \cdot i_{m-1} \cdot i_{m}=j
$$

between $i$ and $j$ is unique. Thus, we must have a cycle $\alpha \beta$ in $>$ such that

$$
\begin{array}{r}
\alpha: o_{i}=o_{i_{0}}^{1}<\ldots<o_{i_{0}}^{x_{0}}<o_{i_{1}}^{1}<\ldots<o_{i_{1}}^{x_{1}}<\ldots<<o_{i_{m}}^{1}<\ldots<o_{i_{m}}^{x_{m}}=o_{j} \\
\beta: o_{i}=\bar{o}_{i_{0}}^{1}>\ldots>\bar{o}_{i_{0}}^{y_{0}}>\bar{o}_{i_{1}}^{1}>\ldots>\bar{o}_{i_{1}}^{y_{1}}>\ldots \ldots>\bar{o}_{i_{m}}^{1}>\ldots>\bar{o}_{i_{m}}^{y_{m}}=o_{j}
\end{array}
$$

where, for $0 \leq k \leq m$, both $x_{k} \geq 1$ and $y_{k} \geq 1$, and each step in both chains $\alpha$ and $\beta$ is directly implied by some "local" relation $>_{l}$ or $>_{l, l^{\prime}}$ constructing $>$.

Without loss of generality, we assume that the cycle in $>$ induced by $\alpha$ and $\beta$ is lengthwise minimal among all such cycles in $>$. In particular, this implies that

(i) for $0 \leq k \leq m$, we have $1 \leq x_{k}, y_{k} \leq 2$ (one object from $O_{i_{k}}$ is required to connect the local relations $>_{i_{k-1}}$ and $>_{i_{k+1}}$, and no more than two elements from $O_{i_{k}}$ are required because $>_{i_{k}}$ is transitively closed),

(ii) for each pair of objects $o \in \alpha, o^{\prime} \in \beta$, we have $o \neq o^{\prime}$, unless $o=o^{\prime}=o_{i}$ or $o=o^{\prime}=o_{j}$, (or otherwise there would be a shorter cycle than $\alpha \beta$ ) and

(iii) for each pair of objects $o \in \alpha, o^{\prime} \in \beta$, no $>_{l}$ (and no $>_{l, l^{\prime}}$ ) implies $o^{\prime}>_{l} o$ (respectively, $o^{\prime}>_{l, l^{\prime}} o$ ), or otherwise, again, there would be a shorter cycle than $\alpha \beta$.

First, let us show that at least one of the chains $\alpha$ and $\beta$ contains at least one internal element. Assume, to the contrary, that both $\alpha$ and $\beta$ contain no internal elements. If $i=j$, then we have $o_{i}>_{i} o_{i}^{\prime}$ (where $o_{i}^{\prime}=o_{j}$ ) and $o_{i}^{\prime}>_{i} o_{i}$, contradicting our assumption that $>_{i}$ is a partial order. (If $>_{i}$ is not a partial order, then neither all $>_{i} \cup>_{i, j}$.) Otherwise, if $i \neq j$, then either $i \in \operatorname{pred}(j)$ or $j \in \operatorname{pred}(i)$. Assuming the latter, $\left(o_{i}>o_{j}\right) \wedge\left(o_{i}>o_{j}\right)$ implies $\left(o_{i}>_{i, j} o_{j}\right) \wedge\left(o_{i}>_{i, j} o_{j}\right)$, contradicting our assumption that $>_{i, j}$ is a partial order.

Given that, let us now prove that $o_{i_{m}}^{x_{m}} \neq \bar{o}_{i_{m}}^{y_{m}}$, contradicting the assumption that the chains $\alpha$ and $\beta$ as in Eq. 40 exist. We do it on a case-by-case basis of possible combinations of $x_{m}, y_{m}$, and length-minimality of the cycle $\alpha \beta$ implies that there are only four such cases to consider.

$\left[x_{m}=2, y_{m}=2\right]$ In this case, Eq. 40 implies $\bar{o}_{i_{m}}^{1}>_{i_{m}} \bar{o}_{i_{m}}^{2}=o_{i_{m}}^{2}>_{i_{m}} o_{i_{m}}^{1}$. The transitivity of $>_{i_{m}}$ then implies $\bar{o}_{i_{m}}^{1}>o_{i_{m}}^{1}$, contradicting our assumption of minimality of the cycle $\alpha \beta$.

$\left[x_{m}=1, y_{m}=1\right]$ From Eq. 39 we have either $i_{m-1} \in \operatorname{pred}\left(i_{m}\right)$ or $i_{m} \in \operatorname{pred}\left(i_{m-1}\right)$. If $i_{m-1} \in \operatorname{pred}\left(i_{m}\right)$, then Eq. 40 implies $\bar{o}_{i_{m-1}}^{y_{m-1}}>_{i_{m}, i_{m-1}} \bar{o}_{i_{m}}^{1}=o_{i_{m}}^{1}>_{i_{m}, i_{m-1}} o_{i_{m-1}}^{x_{m-1}}$. The transitivity of $>_{i_{m}, i_{m-1}}$ then implies $\bar{o}_{i_{m-1}}^{y_{m-1}}>o_{i_{m-1}}^{x_{m-1}}$, contradicting our assumption 
of minimality of the cycle $\alpha \beta$. Otherwise, if $i_{m} \in \operatorname{pred}\left(i_{m-1}\right)$, then Eq. 40 implies $\bar{o}_{i_{m-1}}^{y_{m-1}}>_{i_{m-1}, i_{m}} \bar{o}_{i_{m}}^{1}=o_{i_{m}}^{1}>_{i_{m-1}, i_{m}} o_{i_{m-1}}^{x_{m-1}}$. Again, the transitivity of $>_{i_{m-1}, i_{m}}$ then implies $\bar{o}_{i_{m-1}}^{y_{m-1}}>o_{i_{m-1}}^{x_{m-1}}$, contradicting our assumption of minimality of the cycle $\alpha \beta$.

$\left[x_{m}=2, y_{m}=1\right]$ In this case as well, Eq. 39 implies that we have either $i_{m-1} \in \operatorname{pred}\left(i_{m}\right)$ or $i_{m} \in \operatorname{pred}\left(i_{m-1}\right)$. If $i_{m-1} \in \operatorname{pred}\left(i_{m}\right)$, then Eq. 40 implies $\bar{o}_{i_{m-1}}^{y_{m-1}}>_{i_{m}, i_{m-1}} \bar{o}_{i_{m}}^{1}=$ $o_{i_{m}}^{2}>_{i_{m}} o_{i_{m}}^{1}$. Then, the transitivity of $>_{i_{m}} \cup>_{i_{m}, i_{m-1}}$ implies $\bar{o}_{i_{m-1}}^{y_{m-1}}>o_{i_{m}}^{1}$, contradicting our assumption of minimality of the cycle $\alpha \beta$. Otherwise, if $i_{m} \in \operatorname{pred}\left(i_{m-1}\right)$, then Eq. 40 implies $\bar{o}_{i_{m-1}}^{y_{m-1}}>_{i_{m-1}, i_{m}} \bar{o}_{i_{m}}^{1}=o_{i_{m}}^{2}>_{i_{m}} o_{i_{m}}^{1}$. Then, the transitivity of $>_{i_{m}} \cup>_{i_{m-1}, i_{m}}$ implies $\bar{o}_{i_{m-1}}^{y_{m-1}}>o_{i_{m}}^{1}$, contradicting our assumption of minimality of the cycle $\alpha \beta$.

$\left[x_{m}=1, y_{m}=2\right]$ This case is similar to the previous case of $x_{m}=2, y_{m}=1$, mutatis mutandis.

Theorem 2 Let $\Pi$ be a planning problem in $\mathbf{P}_{b}, \operatorname{COP}_{\Pi}=(\mathcal{X}, \mathcal{F})$ be the corresponding constraint optimization problem, and $\bar{x} \in \operatorname{Dom}(\mathcal{X})$ be an optimal solution for $\mathrm{COP}_{\Pi}$ with $\sum_{\varphi \in \mathcal{F}} \varphi(\bar{x})=\alpha$.

(I) If $\alpha<\infty$, then a plan of cost $\alpha$ for $\Pi$ can be reconstructed from $\bar{x}$ in time polynomial in the description size of $\Pi$.

(II) If $\Pi$ has a plan, then $\alpha<\infty$.

\section{Proof:}

(I) Given a COP solution $\bar{x}=\left\{\tau_{v_{1}}, \ldots, \tau_{v_{n}}\right\}$ with $\sum_{\varphi \in \mathcal{F}} \varphi(\bar{x})=\alpha<\infty$, we construct a plan $\rho$ for $\Pi$ with $\mathcal{C}(\rho)=\alpha$.

First, for each variable $v \in V$ with $\operatorname{pred}(v)=\emptyset$, let a sequence $\rho_{v}$ of actions from $A_{v}$ be defined as

$$
\rho_{v}=\left\{\begin{array}{ll}
\emptyset & \left|\tau_{v}\right|=1 \\
a_{v}^{1} \cdot \ldots \cdot a_{v}^{\left|\tau_{v}\right|-1} & \text { otherwise }
\end{array},\right.
$$

where, for $1 \leq j \leq\left|\tau_{v}\right|-1$,

$$
a_{v}^{j}=\left\{\begin{array}{ll}
a_{\mathbf{b}_{v}}, & j \text { is even } \\
a_{\mathrm{w}_{v}}, & j \text { is odd }
\end{array},\right.
$$

with $\operatorname{eff}\left(a_{\mathbf{b}_{v}}\right)=\left\{\mathrm{b}_{v}\right\}$, and $\operatorname{eff}\left(a_{\mathrm{w}_{v}}\right)=\left\{\mathrm{w}_{v}\right\}$. From Eq. 3 and $\varphi_{v}\left(\tau_{v}\right)<\infty$, we immediately have (i) $\left\{a_{\mathrm{w}_{v}}\right\} \subseteq A_{v}$ if $\left|\tau_{v}\right| \geq 2$, and $\left\{a_{\mathrm{b}_{v}}, a_{\mathrm{w}_{v}}\right\} \subseteq A_{v}$ if $\left|\tau_{v}\right|>2$, and (ii) $\mathcal{C}\left(\rho_{v}\right)=\varphi_{v}\left(\tau_{v}\right)$. Now, for a purpose that gets clear below, let a binary relation $>_{v}$ over the action elements of $\rho_{v}$ be defined as the transitive closure of $\left\{a_{v}^{j-1}<a_{v}^{j}|1<j \leq| \tau_{v} \mid-1\right\}$. Clearly, $>_{v}$ constitutes a strict total ordering over the elements of $\rho_{v}$.

Next, for each non-root variable $v \in V$ with $\operatorname{pred}(v)=\left\{w_{1}, \ldots, w_{k}\right\}$, we construct the graph $G_{e}^{\prime}(v)$ with respect to $\tau_{w_{1}}, \ldots, \tau_{w_{k}}$, and determine in $G_{e}^{\prime}(v)$ a minimal-cost path of 
$\left|\tau_{v}\right|-1$ edges from the source node $\left\langle\mathrm{b}_{w_{1}}^{1} \cdots \mathrm{b}_{w_{k}}^{1}\right\rangle$. The existence of such a path is implied by $\varphi_{v}\left(\tau_{v}, \tau_{w_{1}}, \ldots, \tau_{w_{k}}\right)<\infty$. By the construction of $G_{e}^{\prime}(v)$ we also know that, for $1 \leq j \leq\left|\tau_{v}\right|-$ 1 , the $j$-th edge on this path is from a node labeled with $\left\langle\tau_{w_{1}}\left[l_{1}^{j-1}\right] \cdots \tau_{w_{k}}\left[l_{k}^{j-1}\right]\right\rangle$ to a node labeled with $\left\langle\tau_{w_{1}}\left[l_{1}^{j}\right] \cdots \tau_{w_{k}}\left[l_{k}^{j}\right]\right\rangle$, where for $1 \leq l \leq k$, we have $l_{i}^{0}=1$ and $l_{i}^{j-1} \leq l_{i}^{j}$. Having that, let a sequence $\rho_{v}$ of actions from $A_{v}$ be defined as in Eq. 41, with, for $1 \leq j \leq\left|\tau_{v}\right|-1$,

$$
\begin{aligned}
\operatorname{eff}\left(a_{v}^{j}\right) & =\left\{\tau_{v}[j+1]\right\} \\
\operatorname{pre}\left(a_{v}^{j}\right) & =\left\{\tau_{v}[j], \tau_{w_{1}}\left[l_{1}^{j}\right], \tau_{w_{2}}\left[l_{2}^{j}\right], \ldots, \tau_{w_{k}}\left[l_{k}^{j}\right]\right\}
\end{aligned}
$$

Note that $\left\{a_{v}^{1}, \ldots, a_{v}^{\left|\tau_{v}\right|-1}\right\} \subseteq A_{v}$ is implied by the construction of $G_{e}^{\prime}(v)$ and the presence of the considered minimal-cost path in it.

Now, similarly to the case of the root variables, let a binary relation $>_{v}$ over the action elements of $\rho_{v}$ be defined as the transitive closure of $\left\{a_{v}^{j-1}<a_{v}^{j}|1<j \leq| \tau_{v} \mid-1\right\}$. Here as well, $>_{v}$ constitutes a strict total ordering over the elements of $\rho_{v}$. In addition, for each parent $w_{i}$ of $v$, let a binary relation $>_{v, w_{i}}$ over the union of the action elements of $\rho_{v}$ and $\rho_{w_{i}}$ be defined as the transitive closure of $>_{v, w_{i}}^{-} \cup>_{v, w_{i}}^{+}$, which in turn are defined as

$$
\begin{aligned}
& >_{v, w_{i}}^{-}=\left\{a_{w_{i}}^{l_{i}^{j}-1}<a_{v}^{j}|1 \leq j \leq| \tau_{v} \mid-1, l_{i}^{j}>1\right\} \\
& >_{v, w_{i}}^{+}=\left\{a_{v}^{j}<a_{w_{i}}^{l_{i}^{j}}|1 \leq j \leq| \tau_{v}\left|-1, l_{i}^{j}<\right| \tau_{w_{i}} \mid\right\} .
\end{aligned}
$$

It is not hard to verify from Eq. 44 that, for each $v \in V$ and each $w \in \operatorname{pred}(v)$, not only $>_{v, w}$ constitutes a strict partial ordering, but so are the transitively closed $>_{v} \cup>_{v, w}$ and $>_{w} \cup>_{v, w}$. Given that,

- By the definition of $\rho_{w}=\left\langle a_{w}^{1} \cdot \ldots \cdot a_{w}^{l}\right\rangle$, and the polytree structure of the causal graph $C G(\Pi)$, restricting the preconditions and effects of each $a_{w}^{i}$ to the variables $\{v\} \cup \operatorname{pred}(v)$, we have $\operatorname{pre}\left(a_{w}^{i}\right)=\left\{\mathrm{b}_{w}\right\}, \operatorname{eff}\left(a_{w}^{i}\right)=\left\{\mathrm{w}_{w}\right\}$ for $i$ being odd, and pre $\left(a_{w}^{i}\right)=$ $\left\{\mathrm{w}_{w}\right\}, \operatorname{eff}\left(a_{w}^{i}\right)=\left\{\mathrm{b}_{w}\right\}$ for $i$ being even. For each $1 \leq i \leq k$, from Eq. 43 we have $\operatorname{eff}\left(a_{w_{i}}^{l_{i}^{j}-1}\right) \in \operatorname{pre}\left(a_{v}^{j}\right)$. From Eq. 44 we can now derive that any linearization of $>_{v}$ $\cup \bigcup_{w \in \operatorname{pred}(v)}>_{v, w}$ defines a sequence of actions that is applicable with respect to $\{v\} \cup$ $\operatorname{pred}(v)$. In addition, the construction of the graph $G_{e}^{\prime}(v)$ implies that this action sequence provides to $v$ the value $G[v]$ if the latter is specified.

- The polytree structure of the causal graph $C G(\Pi)$ and Theorem 1 together imply that the transitively closed relation

$$
>=\bigcup_{v \in V}\left(>_{v} \cup \bigcup_{w \in \operatorname{pred}(v)}>_{v, w}\right)
$$

is a strict partial order over the union of the action elements of $\rho_{v_{1}}, \ldots, \rho_{v_{n}}$.

Putting thing together, the above implies that any linearization of $>$ constitutes a valid plan $\rho$ for $\Pi$ with cost

$$
\mathcal{C}(\rho)=\sum_{v \in V} \mathcal{C}\left(\rho_{v}\right)=\sum_{v \in V} \varphi_{v}(\bar{x})
$$


which is exactly what we had to prove. We also note that the plan extraction step of the algorithm polytree-k-indegree corresponds exactly to the above construction along Eqs. 4144 , providing us in polynomial time with a concrete cost-optimal plan corresponding to the optimal solution for $\mathrm{COP}_{\Pi}$.

(II) We now prove that if $\Pi$ is solvable, then we must have $\alpha<\infty$. Assume to the contrary that this is not the case. Let $\Pi$ be a solvable planning problem, and let $\rho$ be an irreducible plan for $\Pi$. Given such $\rho$, let $\bar{x}_{\rho}=\left\{\tau_{v_{1}}, \ldots, \tau_{v_{n}}\right\}$ be an COP assignment with each $\left|\tau_{v_{i}}\right|=\left|\rho \downarrow_{v_{i}}\right|-1$. Note that $\bar{x}_{\rho}$ is well-defined (that is, for $1 \leq i \leq n$, we have $\left.\tau_{v_{i}} \in \unrhd^{*}\left[\tau\left(v_{i}\right)\right]\right)$ by the definition of $\tau\left(v_{i}\right)$, Corollary 1 , and $\rho$ being irreducible. Let us now show that $\sum_{\varphi \in \mathcal{F}} \varphi\left(\bar{x}_{\rho}\right) \leq \mathcal{C}(\rho)$, contradicting our assumption that $\alpha=\infty$ due to $\alpha \leq \sum_{\varphi \in \mathcal{F}} \varphi\left(\bar{x}_{\rho}\right)$ and $\mathcal{C}(\rho)<\infty$.

First, for each variable $v$ with $\operatorname{pred}(v)=\emptyset$, Eq. 3 immediately implies $\varphi_{v}\left(\bar{x}_{\rho}\right) \leq \mathcal{C}\left(\rho \downarrow_{v}\right)$. Next, for each non-root variable $v \in V$ with $\operatorname{pred}(v)=\left\{w_{1}, \ldots, w_{k}\right\}$, consider the graph $G_{e}^{\prime}(v)$ constructed with respect to $\tau_{w_{1}}, \ldots, \tau_{w_{k}}$. Let $\left\{a_{1}, \ldots, a_{\left|\rho \downarrow_{v}\right|}\right\}$ be the actions of $\rho \downarrow_{v}$ numbered in the order of their appearance along $\rho \downarrow_{v}$. Let $\left\{y_{w_{1}}(1), \ldots, y_{w_{k}}(1)\right\}$ denote the prevail condition of $a_{1}$ with each $y_{w_{i}}(1)$ being time-stamped with its earliest appearance along $\tau_{w_{i}}$, that is, $y_{w_{i}}(1) \in\left\{\mathrm{b}_{w_{i}}^{1}, \mathrm{w}_{w_{i}}^{1}\right\}$. Now, for $2 \leq j \leq\left|\rho \downarrow_{v}\right|$, we set $\left\{y_{w_{1}}(j), \ldots, y_{w_{k}}(j)\right\}$ to the prevail condition of $a_{i}$ with each $y_{w_{i}}(j)$ being time-stamped with the lowest possible time index along $\tau_{w_{i}}$ satisfying " $y_{w_{i}}(j-1)$ does not come after $y_{w_{i}}(j)$ along $\tau_{w_{i}}$ ". Given that

(i) $\rho \downarrow_{v}$ is a complete order-preserving restriction of $\rho$ to the $v$-changing actions $A_{v}$,

(ii) the sequence of time-stamped prevail conditions $\left\{\left\{y_{w_{1}}(j), \ldots, y_{w_{k}}(j)\right\}\right\}_{j=1}^{\left|\rho \downarrow_{v}\right|}$ is constructed as above, and

(iii) $\left|\rho \downarrow_{v}\right|=\left|\tau_{v}\right|-1$ by the construction of $\bar{x}_{\rho}$,

we have that $G_{e}^{\prime}(v)$ contains a path

$$
\left\langle\mathrm{b}_{w_{1}}^{1} \cdots \mathrm{b}_{w_{k}}^{1}\right\rangle \rightarrow\left\langle y_{w_{1}}(1) \cdots y_{w_{k}}(1)\right\rangle \rightarrow \ldots \rightarrow\left\langle y_{w_{1}}\left(\left|\rho \downarrow_{v}\right|\right) \cdots y_{w_{k}}\left(\left|\rho \downarrow_{v}\right|\right)\right\rangle
$$

and the cost of this path is $\mathcal{C}\left(\rho \downarrow_{v}\right)<\infty$. However, from the constructive definition of $\varphi_{v}$ in the algorithm polytree-k-indegree, we have $\varphi_{v}\left(\bar{x}_{\rho}\right)$ being the cost of the minimal-cost path of $\left|\tau_{v}\right|-1$ edges in $G_{e}^{\prime}(v)$ originated in $\left\langle\mathrm{b}_{w_{1}}^{1} \cdots \mathrm{b}_{w_{k}}^{1}\right\rangle$, and thus $\varphi_{v}\left(\bar{x}_{\rho}\right) \leq \mathcal{C}\left(\rho \downarrow_{v}\right)$. The latter argument is valid for all planning variables $v \in V$, and thus we have

$$
\sum_{\varphi \in \mathcal{F}} \varphi\left(\bar{x}_{\rho}\right) \leq \sum_{v \in V} \mathcal{C}\left(\rho \downarrow_{v}\right)=\mathcal{C}(\rho)
$$

which is what we had to prove.

Theorem 6 Let $\Pi$ be a $\mathbf{P}(1)$ problem with uniform-costs actions, $\operatorname{COP}_{\Pi}=(\mathcal{X}, \mathcal{F})$ be the corresponding constraint optimization problem, and $\bar{x}$ be an optimal assignment to $\mathcal{X}$ with $\sum_{\varphi \in \mathcal{F}} \varphi(\bar{x})=\alpha$. 
(I) If $\alpha<\infty$, then a plan of cost $\alpha$ for $\Pi$ can be reconstructed from $\bar{x}$ in time polynomial in the description size of $\Pi$.

(II) If $\Pi$ has a plan, then $\alpha<\infty$.

\section{Proof:}

(I) Given a COP solution $\bar{x}$ with $\sum_{\varphi \in \mathcal{F}} \varphi(\bar{x})=\alpha<\infty$, we construct a plan $\rho$ for $\Pi$ with $\mathcal{C}(\rho)=\alpha$. We construct this plan by

1. Traversing the planning variables in a topological ordering of the causal graph $C G(\Pi)$, and associating each variable $v$ with a sequence $\rho_{v} \in A_{v}^{*}$.

2. Merging the constructed sequences $\rho_{v_{1}}, \ldots, \rho_{v_{n}}$ into the desired plan $\rho$.

For each variable $x_{v} \in \mathcal{X}$, let $\sigma_{v}$ denote the value provided by $\bar{x}$ to $x_{v}$. First, for each variable $v \in V$ with $\operatorname{pred}(v)=\emptyset$, let a sequence $\rho_{v}$ of actions from $A_{v}$ be defined as

$$
\rho_{v}=\left\{\begin{array}{ll}
\emptyset & \left|\sigma_{v}\right|=1 \\
a_{v}^{1} \cdot \ldots \cdot a_{v}^{\left|\sigma_{v}\right|-1} & \text { otherwise }
\end{array},\right.
$$

where, for $1 \leq j \leq\left|\sigma_{v}\right|-1$,

$$
a_{v}^{j}=\left\{\begin{array}{ll}
a_{\mathbf{b}_{v}}, & j \text { is even } \\
a_{\mathrm{w}_{v}}, & j \text { is odd }
\end{array},\right.
$$

with $\operatorname{eff}\left(a_{\mathbf{b}_{v}}\right)=\left\{\mathrm{b}_{v}\right\}$, and $\operatorname{eff}\left(a_{\mathrm{w}_{v}}\right)=\left\{\mathrm{w}_{v}\right\}$. From Eq. 14 and $\varphi_{v}\left(\sigma_{v}\right) \leq \alpha<\infty$, we immediately have (i) $\left\{a_{\mathrm{w}_{v}}\right\} \subseteq A_{v}$ if $\left|\sigma_{v}\right| \geq 2$, and $\left\{a_{\mathrm{b}_{v}}, a_{\mathrm{w}_{v}}\right\} \subseteq A_{v}$ if $\left|\sigma_{v}\right|>2$, and (ii) $\mathcal{C}\left(\rho_{v}\right)=\varphi_{v}\left(\sigma_{v}\right)$. Let a binary relation $>_{v}$ over the action elements of $\rho_{v}$ be defined as the transitive closure of $\left\{a_{v}^{j-1}<a_{v}^{j}|1<j \leq| \sigma_{v} \mid-1\right\}$, that is

$$
>_{v}=\left\{a_{v}^{j^{\prime}}<a_{v}^{j}\left|1 \leq j^{\prime}<j \leq\right| \sigma_{v} \mid-1\right\}
$$

Clearly, $>_{v}$ constitutes a strict total ordering over the elements of $\rho_{v}$, making $\rho_{v}$ an applicable sequence of actions that provides to $v$ the value $G[v]$ if the latter is specified.

Next, for each variable $v \in V$ with $\operatorname{pred}(v) \neq \emptyset$, let $\operatorname{pred}(v)=\left\{w_{1}, \ldots, w_{k}\right\}$ be numbered according to their ordering used for constructing $\operatorname{COP}_{\Pi}$. Likewise, for each $w_{i} \in \operatorname{pred}(v)$, let $\left[\delta_{\mathrm{w}}(i), \delta_{\mathrm{b}}(i), \eta(i)\right]$ be the value provided by $\bar{x}$ to $x_{v}^{w_{i}}$. Given that, let a pair of indexes $0 \leq\langle\mathrm{w}\rangle,\langle\mathrm{b}\rangle \leq k$ be defined as

$$
\begin{aligned}
& \langle\mathrm{w}\rangle= \begin{cases}0, & \delta_{\mathrm{w}}(k)=0, \\
1, & \delta_{\mathrm{w}}(1)=1, \\
j, & \delta_{\mathrm{w}}(j-1)<\delta_{\mathrm{w}}(j), 2 \leq j \leq k\end{cases} \\
& \langle\mathrm{b}\rangle= \begin{cases}0, & \delta_{\mathrm{b}}(k)=0, \\
1, & \delta_{\mathrm{b}}(1)=1, \\
j, & \delta_{\mathrm{b}}(j-1)<\delta_{\mathrm{b}}(j), 2 \leq j \leq k\end{cases}
\end{aligned}
$$


In other words, $\langle\mathrm{w}\rangle$ captures the smallest $1 \leq j \leq k$ such that $\delta_{\mathrm{w}}(j)=1$, and 0 , if there is no such $j$ at all; the semantics of $\langle\mathrm{b}\rangle$ is similar, mutatis mutandis.

Informally, in our next-coming construction of the action sequence $\rho_{v}$ for the state variable $v,\langle\mathrm{w}\rangle$ and $\langle\mathrm{b}\rangle$ will indicate the parents prevailing the value changes of $v$ to $\mathrm{w}_{v}$ and to $\mathrm{b}_{v}$, respectively, along $\rho_{v}$. Note that Eqs. 48-49 are well-defined because, for $2 \leq j \leq k$, Eq. 18 implies

$$
\delta_{\mathrm{w}}(j-1) \leq \delta_{\mathrm{w}}(j) \wedge \delta_{\mathrm{b}}(j-1) \leq \delta_{\mathrm{b}}(j) \wedge \eta(j-1)=\eta(j) .
$$

Given this notation, the action sequence $\rho_{v}$ and the partial orders $>_{v, w_{1}}, \ldots,>_{v, w_{k}}$ are constructed as follows.

$[\langle\mathbf{w}\rangle=0,\langle\mathbf{b}\rangle=0]$ In this case, the constructed plan $\rho$ should perform no value changes of $v$, and thus $\rho_{v}$ is set to an empty action sequence, and, consequently, both $>_{v}$ and all $>_{v, w}$ are set to empty sets.

$[\langle w\rangle>0,\langle b\rangle=0]$ In this case, the constructed plan $\rho$ should perform exactly one value change of $v$ (from $\mathrm{b}_{v}$ to $\mathrm{w}_{v}$ ), and thus $\rho_{v}$ is set to contain exactly one action $a_{v}^{1}$ with $\operatorname{eff}(a)=\left\{w_{v}\right\}$, and

$$
\operatorname{pre}\left(a_{v}^{1}\right)= \begin{cases}\left\{\mathrm{b}_{v}, \mathrm{~b}_{\left.w_{\langle w\rangle}\right\}}\right\}, & a_{\mathrm{w}_{v} \mid \mathrm{b}_{w_{\langle\mathrm{w}}}} \in A_{v} \\ \left\{\mathrm{~b}_{v}, \mathrm{w}_{w_{\langle\mathrm{w}\rangle}}\right\}, & \text { otherwise }\end{cases}
$$

Note that $a_{v}^{1}$ is well-defined, as $\alpha<\infty$ and Eq. 16 together imply that $\left\{a_{\mathrm{w}_{v} \mid \mathrm{b}_{w_{\langle\mathrm{w}\rangle}}}, a_{\mathrm{w}_{v} \mid \mathrm{b}_{w_{\langle\mathrm{w}\rangle}}}\right\} \cap$ $A_{v} \neq \emptyset$ (see case (2) in Eq. 16). In both outcomes of Eq. 50, we set $>_{v}=\emptyset$. If $a_{v}^{1}=a_{\mathrm{w}_{v} \mid \mathrm{b}_{w_{\langle\mathrm{w}\rangle}}}$, we set

$$
>_{v, w\langle\mathrm{w}\rangle}=\left\{a_{v}^{1}<a_{w_{\langle\mathrm{w}\rangle}}^{1} \mid a_{w_{\langle\mathrm{w}\rangle}}^{1} \in \rho_{w_{\langle\mathrm{w}\rangle}}\right\}
$$

Otherwise, if $a_{v}^{1}=a_{\mathrm{w}_{v} \mid \mathrm{w}_{w_{\langle\mathrm{w}\rangle}}}$, then from case (2) in Eq. 16, $a_{\mathrm{w}_{v} \mid \mathrm{b}_{\left.w_{\langle\mathrm{w}\rangle}\right\rangle}} \notin A_{v}$, and $\alpha<\infty$, we have $\left|\sigma_{w_{\langle w\rangle} \mid}\right|>1$, and thus $\left|\rho_{w_{\langle w\rangle}}\right| \geq 1$. Given that, we set

$$
>_{v, w_{\langle\mathrm{w}\rangle}}=\left\{a_{w_{\langle\mathrm{w}\rangle}}^{1}<a_{v}^{1}\right\} \cup\left\{a_{v}^{1}<a_{w_{\langle\mathrm{w}\rangle}}^{2} \mid a_{w_{\langle\mathrm{w}\rangle}}^{2} \in \rho_{w_{\langle\mathrm{w}\rangle}}\right\}
$$

In both cases, it is easy to verify that $>_{v} \cup>_{v, w_{\langle w\rangle}} \cup>_{w_{\langle w\rangle}}$ constitutes a strict total order over the action elements of $\rho_{v}$ and $\rho_{w_{\langle w\rangle}}$. (In particular, this trivially implies that $>_{v} \cup>_{v, w}$ and $>_{v, w} \cup>_{w}$ are strict partial orderings over their domains.)

From Eqs. 47, 51, and 52 we can now derive that any linearization of $>_{v} \cup \bigcup_{w \in \operatorname{pred}(v)}>_{v, w}$ defines a sequence of actions that is applicable with respect to $\{v\} \cup \operatorname{pred}(v)$. In addition, Eq. 12 implies that this action sequence provides to $v$ the value $G[v]$ if the latter is specified.

$[\langle w\rangle>0,\langle b\rangle>0,\langle w\rangle=\langle b\rangle]$ In this case, the constructed plan $\rho$ should perform more than one value change of $v$, and all these value changes should be performed by (a pair of types of) actions prevailed by the value of $w_{\langle w\rangle}$. From $\alpha<\infty$, we have $\varphi\left(\left[\delta_{\mathrm{w}}(\langle\mathrm{w}\rangle), \delta_{\mathrm{b}}(\langle\mathrm{w}\rangle), \eta\right], \sigma_{w\langle\mathrm{w}\rangle}\right)=0$. The specification of the case in question (that is, $\langle w\rangle=\langle b\rangle>0$ ) thus implies that one of the conditions of the cases (4-6) of Eq. 16 should hold. Given that, we distinguish between the following four settings. 
(1) If $\left\{a_{\mathrm{w}_{v} \mid \mathbf{b}_{w_{\langle\mathrm{w}\rangle}}}, a_{\mathrm{b}_{v} \mid \mathbf{b}_{w_{\langle\mathrm{w}\rangle}}}\right\} \subseteq A_{v}$, then $\rho_{v}$ is specified according to Eq. 45, and its action elements are specified as

$$
a_{v}^{i}=\left\{\begin{array}{ll}
a_{\mathrm{w}_{v} \mid \mathrm{b}_{\left.w_{\langle\mathrm{w}\rangle}\right\rangle},}, \quad i \text { is odd } \\
a_{\mathrm{b}_{v} \mid \mathrm{b}_{w_{\langle\mathrm{w}\rangle}},}, \quad i \text { is even }
\end{array} .\right.
$$

The relation $>_{v}$ is set according to Eq. 47 , and $>_{v, w_{\langle\mathrm{w}\rangle}}$ is set to

$$
>_{v, w\langle w\rangle}=\left\{a_{v}^{i}<a_{w_{\langle\mathrm{w}\rangle}}^{1} \mid a_{v}^{i} \in \rho_{v}, a_{w_{\langle\mathrm{w}\rangle}}^{1} \in \rho_{w_{\langle\mathrm{w}\rangle}}\right\}
$$

Finally, for all $w \in \operatorname{pred}(v) \backslash\left\{w_{\langle\mathrm{w}\rangle}\right\}$, we set $>_{v, w}=\emptyset$.

(2) Otherwise, if $\left\{a_{\mathrm{w}_{v} \mid \mathrm{w}_{w_{\langle\mathrm{w}\rangle}}}, a_{\mathrm{b}_{v}\left|\mathrm{w}_{\left.w_{\langle}\right\rangle}\right\rangle}\right\} \subseteq A_{v}$ and $\left|\sigma_{\left.w_{\langle\mathrm{w}\rangle}\right\rangle}\right|>1$, then we have $\left|\rho_{w_{\langle\mathrm{w}\rangle}}\right| \geq 1$. Given that, we again set $\rho_{v}$ according to Eq. 45 , but now with its action elements being set as

$$
a_{v}^{i}=\left\{\begin{array}{ll}
a_{\left.\mathrm{w}_{v}\left|\mathrm{w}_{w}\right\rangle\right\rangle}, & i \text { is odd } \\
a_{\mathrm{b}_{v} \mid \mathrm{w}_{\left.w_{\langle w}\right\rangle}}, & i \text { is even }
\end{array} .\right.
$$

The relation $>_{v}$ is set according to Eq. 47 , and $>_{v, w\langle w\rangle}$ is set to

$$
>_{v, w_{\langle\mathrm{w}\rangle}}=\left\{a_{w_{\langle\mathrm{w}\rangle}}^{1}<a_{v}^{i} \mid a_{v}^{i} \in \rho_{v}\right\} \cup\left\{a_{v}^{i}<a_{w_{\langle\mathrm{w}\rangle}}^{2} \mid a_{v}^{i} \in \rho_{v}, a_{w_{\langle\mathrm{w}\rangle}}^{2} \in \rho_{w_{\langle\mathrm{w}\rangle}}\right\}
$$

Finally, for all $w \in \operatorname{pred}(v) \backslash\left\{w_{\langle\mathrm{w}\rangle}\right\}$, we set $>_{v, w}=\emptyset$.

(3) Otherwise, if $\left\{a_{\mathrm{w}_{v} \mid \mathbf{b}_{w_{\langle w\rangle}}}, a_{\mathbf{b}_{v} \mid \mathrm{w}_{\left.w_{\langle w}\right\rangle}}\right\} \subseteq A_{v}$, and $\left|\sigma_{w_{\langle\mathrm{w}\rangle}}\right| \geq\left|\sigma_{v}\right|-1$, then $\rho_{v}$ is specified according to Eq. 45, and its action elements are specified as

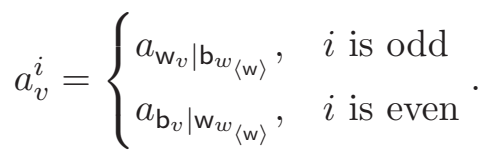

The relation $>_{v}$ is set according to Eq. 47 , and $>_{v, w_{\langle w\rangle}}$ is set to

$$
>_{v, w_{\langle\mathrm{w}\rangle}}=\bigcup_{a_{v}^{i} \in \rho_{v}, a_{w_{\langle\mathrm{w}\rangle}}^{j} \in \rho_{w_{\langle\mathrm{w}\rangle}}}\left\{a_{v}^{i}<a_{w_{\langle\mathrm{w}\rangle}}^{j} \mid i \leq j\right\} \cup\left\{a_{w_{\langle\mathrm{w}\rangle}}^{j}<a_{v}^{i} \mid i>j\right\}
$$

For all $w \in \operatorname{pred}(v) \backslash\left\{w_{\langle\mathrm{w}\rangle}\right\}$, we set $>_{v, w}=\emptyset$.

(4) Otherwise, if $\left\{a_{\mathrm{w}_{v} \mid \mathrm{w}_{w_{1}}}, a_{\mathbf{b}_{v} \mid \mathbf{b}_{w_{1}}}\right\} \subseteq A_{v}$, and $\left|\sigma_{\left.w_{\langle\mathrm{w}\rangle}\right\rangle}\right| \geq\left|\sigma_{v}\right|$, then $\rho_{v}$ is specified according to Eq. 45, and its action elements are specified as

$$
a_{v}^{i}=\left\{\begin{array}{ll}
a_{\mathrm{w}_{v} \mid \mathrm{w}_{w}}, & i \text { is odd } \\
a_{\mathrm{b}_{v} \mid \mathrm{b}_{w_{\langle\mathrm{w}\rangle}},}, & i \text { is even }
\end{array} .\right.
$$

The relation $>_{v}$ is set according to Eq. 47 , and $>_{v, w}$ iw is set to

$$
>_{v, w_{\langle\mathrm{w}\rangle}}=\bigcup_{a_{v}^{i} \in \rho_{v}, a_{w_{\langle\mathrm{w}\rangle}}^{j} \in \rho_{w_{\langle\mathrm{w}\rangle}}}\left\{a_{v}^{i}<a_{w_{\langle\mathrm{w}\rangle}}^{j} \mid i<j\right\} \cup\left\{a_{w_{\langle\mathrm{w}\rangle}}^{j}<a_{v}^{i} \mid i \geq j\right\}
$$

For all $w \in \operatorname{pred}(v) \backslash\left\{w_{\langle\mathrm{w}\rangle}\right\}$, we set $>_{v, w}=\emptyset$. 
In all the four cases above, $>_{v} \cup>_{v, w_{\langle w\rangle}} \cup>_{w_{\langle w\rangle}}$ constitutes a strict total order over the elements of $\rho_{v}$ and $\rho_{w_{\langle w\rangle}}$.

From Eqs. 47, 54, 56, and 58, 60 we can now derive that any linearization of $>_{v}$ $\cup \bigcup_{w \in \operatorname{pred}(v)}>_{v, w}$ defines a sequence of actions that is applicable with respect to $\{v\} \cup$ $\operatorname{pred}(v)$. In addition, Eq. 12 implies that this action sequence provides to $v$ the value $G[v]$ if the latter is specified.

$[\langle w\rangle>0,\langle b\rangle>0,\langle w\rangle \neq\langle b\rangle]$ In this case, the constructed plan $\rho$ should perform more than one value change of $v$, with changes of $v$ to $\mathrm{w}_{v}$ and $\mathrm{b}_{v}$ being performed by (a pair of types of) actions prevailed by the value of $w_{\langle\mathrm{w}\rangle}$ and $w_{\langle\mathbf{b}\rangle}$, respectively. From $\alpha<\infty$, we have $\left.\varphi\left(\left[\delta_{\mathrm{w}}(\langle\mathrm{w}\rangle), \delta_{\mathrm{b}}(\langle\mathrm{w}\rangle), \eta\right], \sigma_{\left.w_{\langle\mathrm{w}\rangle}\right\rangle}\right)=\varphi\left(\llbracket \delta_{\mathrm{w}}(\langle\mathrm{b}\rangle), \delta_{\mathrm{b}}(\langle\mathrm{b}\rangle), \eta\right], \sigma_{w_{\langle\mathrm{b}\rangle}}\right)=0$, and this is due to the respective satisfaction of the conditions of cases (2) and (3) in Eq. 16. Given that, we distinguish between the following four settings ${ }^{5}$.

(1) If $\left\{a_{\mathrm{w}_{v} \mid \mathbf{b}_{w_{\langle\mathrm{w}\rangle}}}, a_{\mathrm{b}_{v} \mid \mathbf{b}_{w_{\langle\mathbf{b}\rangle}}}\right\} \subseteq A_{v}$, then $\rho_{v}$ is specified according to Eq. 45, and its action elements are specified as

$$
a_{v}^{i}=\left\{\begin{array}{ll}
a_{\mathrm{w}_{v} \mid \mathrm{b}_{w}\langle\mathrm{w}\rangle}, & i \text { is odd } \\
a_{\mathrm{b}_{v} \mid \mathrm{b}_{w_{\langle\mathrm{b}\rangle}},}, & i \text { is even }
\end{array} .\right.
$$

The relation $>_{v}$ over the action elements of $\rho_{v}$ is set according to Eq. 47, the relation $>_{v, w_{\langle w\rangle}}$ over the action elements of $\rho_{v}$ and $\rho_{w_{\langle\mathrm{w}\rangle}}$ is set to

$$
>_{v, w\langle w\rangle}=\left\{a_{v}^{i}<a_{w_{\langle\mathrm{w}\rangle}}^{1} \mid i \text { is odd, } a_{v}^{i} \in \rho_{v}, a_{w_{\langle\mathrm{w}\rangle}}^{1} \in \rho_{w_{\langle\mathrm{w}\rangle}}\right\}
$$

and the relation $>_{v, w_{\langle\mathrm{b}\rangle}}$ over the action elements of $\rho_{v}$ and $\rho_{w_{\langle\mathrm{b}\rangle}}$ is set to

$$
>_{v, w_{\langle\mathrm{b}\rangle}}=\left\{a_{v}^{i}<a_{w_{\langle\mathrm{b}\rangle}}^{1} \mid i \text { is even, } a_{v}^{i} \in \rho_{v}, a_{w_{\langle\mathrm{b}\rangle}}^{1} \in \rho_{w_{\langle\mathrm{b}\rangle}}\right\}
$$

For all $w \in \operatorname{pred}(v) \backslash\left\{w_{\langle\mathbf{w}\rangle}, w_{\langle\mathbf{b}\rangle}\right\}$, we set $>_{v, w}=\emptyset$.

(2) Otherwise, if $\left\{a_{\mathrm{w}_{v} \mid \mathrm{w}_{\left.w_{w}\right\rangle}}, a_{\mathbf{b}_{v} \mid \mathbf{b}_{w_{\langle\mathbf{b}\rangle}}}\right\} \subseteq A_{v}$ and $\left|\sigma_{w_{\langle\mathrm{w}\rangle}}\right|>1$, then we have $\left|\rho_{w_{\langle\mathrm{w}\rangle}}\right| \geq 1$. Given that, we again set $\rho_{v}$ according to Eq. 45 , but now with its action elements being set as

$$
a_{v}^{i}=\left\{\begin{array}{ll}
a_{\mathrm{w}_{v} \mid \mathrm{w}_{w}}, & i \text { is odd } \\
a_{\mathrm{b}_{v} \mid \mathrm{b}_{\left.w_{\langle\mathrm{b}}\right\rangle}}, & i \text { is even }
\end{array} .\right.
$$

The relation $>_{v}$ is set according to Eq. $47,>_{v, w\langle w\rangle}$ is set to

$$
>_{v, w_{\langle\mathrm{w}\rangle}}=\bigcup_{a_{v}^{i} \in \rho_{v}, i \text { is odd }}\left\{a_{w_{\langle\mathrm{w}\rangle}}^{1}<a_{v}^{i}\right\} \cup\left\{a_{v}^{i}<a_{w_{\langle\mathrm{w}\rangle}}^{2} \mid a_{w_{\langle\mathrm{w}\rangle}}^{2} \in \rho_{w_{\langle\mathrm{w}\rangle}}\right\}
$$

and $>_{v, w_{\langle\mathbf{b}\rangle}}$ is set to

$$
>_{v, w_{\langle\mathrm{b}\rangle}}=\left\{a_{v}^{i}<a_{w_{\langle\mathrm{b}\rangle}}^{1} \mid i \text { is even, } a_{v}^{i} \in \rho_{v}, a_{w_{\langle\mathrm{b}\rangle}}^{1} \in \rho_{w_{\langle\mathrm{b}\rangle}}\right\}
$$

For all $w \in \operatorname{pred}(v) \backslash\left\{w_{\langle\mathbf{w}\rangle}, w_{\langle\mathbf{b}\rangle}\right\}$, we set $>_{v, w}=\emptyset$.

5. While the details are slightly different, the four settings in this case are conceptually similar to these in the previously considered case of $\langle w\rangle>0,\langle b\rangle>0,\langle w\rangle=\langle b\rangle$. 
(3) Otherwise, if $\left\{a_{\mathbf{b}_{v} \mid w_{w_{\langle\mathbf{b}\rangle}}}, a_{\mathbf{w}_{v} \mid \mathbf{b}_{w_{\langle\mathbf{w}\rangle}}}\right\} \subseteq A_{v}$, and $\left|\sigma_{w_{\langle\mathbf{b}\rangle}}\right|>1$, then we have $\left|\rho_{w_{\langle\mathbf{b}\rangle}}\right| \geq 1$. Given that, we $\rho_{v}$ is specified according to Eq. 45 , and its action elements are specified as

$$
a_{v}^{i}=\left\{\begin{array}{ll}
a_{\mathrm{w}_{v} \mid \mathrm{b}_{\left.w_{\langle\mathrm{w}}\right\rangle}}, & i \text { is odd } \\
a_{\mathrm{b}_{v} \mid \mathrm{w}_{w_{\langle\mathrm{b}\rangle}},}, & i \text { is even }
\end{array} .\right.
$$

The relation $>_{v}$ is set according to Eq. $47,>_{v, w_{\langle w\rangle}}$ is set to

$$
>_{v, w_{\langle\mathrm{w}\rangle}}=\left\{a_{v}^{i}<a_{w_{\langle\mathrm{w}\rangle}}^{1} \mid i \text { is odd, } a_{v}^{i} \in \rho_{v}, a_{w_{\langle\mathrm{w}\rangle}}^{1} \in \rho_{w_{\langle\mathrm{w}\rangle}}\right\}
$$

and $>_{v, w_{\langle\mathbf{b}\rangle}}$ is set to

$$
>_{v, w_{\langle\mathrm{b}\rangle}}=\bigcup_{a_{v}^{i} \in \rho_{v}, i \text { is even }}\left\{a_{w_{\langle\mathrm{b}\rangle}}^{1}<a_{v}^{i}\right\} \cup\left\{a_{v}^{i}<a_{w_{\langle\mathrm{b}\rangle}}^{2} \mid a_{w_{\langle\mathrm{b}\rangle}}^{2} \in \rho_{w_{\langle\mathrm{b}\rangle}}\right\}
$$

For all $w \in \operatorname{pred}(v) \backslash\left\{w_{\langle\mathrm{w}\rangle}\right\}$, we set $>_{v, w}=\emptyset$.

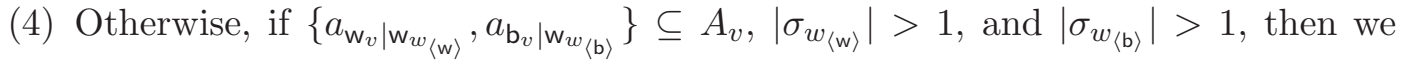
have both $\left|\rho_{w_{\langle\mathbf{w}\rangle}}\right| \geq 1$ and $\left|\rho_{w_{\langle\mathbf{b}\rangle}}\right| \geq 1$. Given that, we again set $\rho_{v}$ according to Eq. 45, and its action elements are specified as

$$
a_{v}^{i}=\left\{\begin{array}{ll}
a_{\mathrm{w}_{v} \mid \mathrm{w}_{\left.w_{\langle\mathrm{w}}\right\rangle},}, \quad i \text { is odd } \\
a_{\mathrm{b}_{v} \mid \mathrm{w}_{w_{\langle\mathrm{b}\rangle}},}, \quad i \text { is even }
\end{array} .\right.
$$

The relation $>_{v}$ is set according to Eq. $47,>_{v, w_{\langle w\rangle}}$ is set to

$$
>_{v, w_{\langle w\rangle}}=\bigcup_{a_{v}^{i} \in \rho_{v}, i \text { is odd }}\left\{a_{w_{\langle\mathrm{w}\rangle}}^{1}<a_{v}^{i}\right\} \cup\left\{a_{v}^{i}<a_{w_{\langle\mathrm{w}\rangle}}^{2} \mid a_{w_{\langle\mathrm{w}\rangle}}^{2} \in \rho_{w_{\langle\mathrm{w}\rangle}}\right\}
$$

and $>_{v, w_{\langle\mathbf{b}\rangle}}$ is set to

$$
>_{v, w_{\langle\mathbf{b}\rangle}}=\bigcup_{a_{v}^{i} \in \rho_{v}, i \text { is even }}\left\{a_{w_{\langle\mathbf{b}\rangle}}^{1}<a_{v}^{i}\right\} \cup\left\{a_{v}^{i}<a_{w_{\langle\mathbf{b}\rangle}}^{2} \mid a_{w_{\langle\mathbf{b}\rangle}}^{2} \in \rho_{w_{\langle\mathbf{b}\rangle}}\right\}
$$

For all $w \in \operatorname{pred}(v) \backslash\left\{w_{\langle\mathbf{w}\rangle}\right\}$, we set $>_{v, w}=\emptyset$.

In all the four cases above, both $>_{v} \cup>_{v, w_{\langle\mathrm{w}\rangle}} \cup>_{w_{\langle\mathrm{w}\rangle}}$ and $>_{v} \cup>_{v, w_{\langle\mathrm{b}\rangle}} \cup>_{w_{\langle\mathrm{b}\rangle}}$ constitute strict total orders over their respective domains.

From Eqs. 47, 62, 63, 65, 66, 68, 69, 71, and 72 we can now derive that any linearization of $>_{v} \cup \bigcup_{w \in \operatorname{pred}(v)}>_{v, w}$ defines a sequence of actions that is applicable with respect to $\{v\} \cup \operatorname{pred}(v)$. In addition, Eq. 12 implies that this action sequence provides to $v$ the value $G[v]$ if the latter is specified.

Until now, for each variable $v \in V$, we have specified an action sequence $\rho_{v}$ and the order $>_{v}$ over the elements of $\rho_{v}$. For each $w \in \operatorname{pred}(v)$, we have specified the order $>_{v, w}$, and proved that all $>_{v} \cup>_{v, w}$ and $>_{w} \cup>_{v, w}$ form strict partial orders over their domains, 
and any linearization of $>_{v} \cup \bigcup_{w \in \operatorname{pred}(v)}>_{v, w}$ defines a sequence of actions that is applicable with respect to $\{v\} \cup \operatorname{pred}(v)$ and provides to $v$ the value $G[v]$ if the latter is specified. This construction allows us to apply now Theorem 1 on the (considered as sets) sequences $\rho_{v}$ and orders $>_{v}$ and $>_{v, w}$, proving that

$$
>=\bigcup_{v \in V}\left(>_{v} \cup \bigcup_{w \in \operatorname{pred}(v)}>_{v, w}\right)
$$

forms a strict partial order over the union of $\rho_{v_{1}}, \ldots, \rho_{v_{n}}$.

Here we also note that the plan extraction step of the algorithm polytree-1-dep-uniform corresponds exactly to the above construction along Eqs. 45-72, providing us in polynomial time with a concrete cost-optimal plan corresponding to the optimal solution for $\mathrm{COP}_{\Pi}$.

(II) We now prove that if $\Pi$ is solvable, then we must have $\alpha<\infty$. Assume to the contrary that this is not the case. Let $\Pi$ be a solvable $\mathbf{P}(1)$ problem, and let (using Theorem 5) $\rho$ be an irreducible, post-unique plan for $\Pi$. Given such $\rho$, let a COP assignment $\bar{x}_{\rho}$ be defined as follows.

1. For each COP variable $x_{v}$, the assignment $\bar{x}_{\rho}$ provides the value $\sigma_{v} \in \unrhd^{*}[\sigma(v)]$ such that $\left|\sigma_{v}\right|=\left|\rho \downarrow_{v}\right|+1$.

2. For each COP variable $x_{v}^{w_{i}}$, the assignment $\bar{x}_{\rho}$ provides the value $\llbracket \delta_{\mathrm{w}_{v}}^{w}, \delta_{\mathrm{b}_{v}}^{w},\left|\sigma_{v}\right|-1 \rrbracket$, where $\delta_{\mathrm{w}_{v}}^{w}=1$ just if some action in $\rho \downarrow_{v}$ changes the value of $v$ to $\mathrm{w}_{v}$ while (considering the pre-fixed ordering of the $v$ 's parents) being preconditioned by the value of some $w_{j}, j \leq i$, and $\delta_{\mathbf{w}_{v}}^{w}=0$, otherwise. $\delta_{\mathbf{b}_{v}}^{w}$ is defined similarly to $\delta_{\mathbf{w}_{v}}^{w}$, mutatis mutandis.

From Eq. 14-18 we then directly have that, for all $v \in V, \varphi_{x_{v}}\left(\bar{x}_{\rho}\right)=\left|\rho \downarrow_{v}\right|$, and for all $w \in \operatorname{pred}(v), \varphi_{x_{v}^{w}}\left(\bar{x}_{\rho}\right)=0$. Therefore, we have

$$
\sum_{\varphi \in \mathcal{F}} \varphi\left(\bar{x}_{\rho}\right)=\sum_{v \in V} \mathcal{C}\left(\rho \downarrow_{v}\right)=\mathcal{C}(\rho)
$$

which is what we had to prove.

Theorem 8 For every solvable $\mathbf{P}(1)$ problem $\Pi=(V, A, I, G)$, the plan set $\mathcal{P}^{3 / 2}(\Pi)$ contains at least one cost-optimal plan.

Proof: Given a $\mathbf{P}(1)$ problem $\Pi=(V, A, I, G)$, and cost-optimal plan $\rho$ for $\Pi$, we construct a sequence of actions $\rho^{*}$ such that:

- $\rho^{*}$ is a post-3/2 plan for $\Pi$,

- $\mathcal{C}\left(\rho^{*}\right)=\mathcal{C}(\rho)$.

In nutshell, first, for each $v \in V$, we map the subsequence $\rho \downarrow_{v}=\left\langle a_{1}, \ldots, a_{k}\right\rangle$ of $\rho$ into a sequence of actions $\rho_{v}^{*}=\left\langle a_{1}^{*}, \ldots, a_{k}^{*}\right\rangle$ that (i) satisfy the post-3/2 property, and (ii) $\mathcal{C}\left(\rho_{v}^{*}\right) \leq \mathcal{C}\left(\rho \downarrow_{v}\right)$. Then, we merge the constructed sequences $\left\{\rho_{v}^{*}\right\}_{v \in V}$ into $\rho^{*}$, and show that $\rho^{*}$ is a valid plan for $\Pi$. The two properties of $\rho^{*}$ as required above will then hold 
immediately because $\mathcal{C}\left(\rho^{*}\right)=\mathcal{C}(\rho)$, and $\rho^{*}$ being post-3/2 is implied by all its per-variable components $\rho_{v}^{*}$ being post- $3 / 2$.

For each variable $v \in V$, with $\operatorname{pred}(v)=\emptyset$, we set $\rho_{v}^{*}=\rho \downarrow_{v}$ and

$$
>_{v}=\left\{a_{i}<a_{j} \mid a_{i}, a_{j} \in \rho \downarrow_{v}, i<j\right\} .
$$

It is immediate from Eq. 73 that $>_{v}$ is a strict total order over the elements of $\rho_{v}^{*}$.

In turn, for each variable $v \in V$ with $\operatorname{pred}(v) \neq \emptyset$, given $\left\{\sigma_{w}\right\}_{w \in \operatorname{pred}(v)}$, such that $\left|\sigma_{w}\right|=\left|\rho \downarrow_{w}\right|+1$, let $a_{i}^{\alpha}$ be the $i$ 'th cheapest action that changes variable $v$ to $\alpha \in\left\{\mathrm{b}_{v}, \mathrm{w}_{v}\right\}$ and prevailed by some value from $\left\{\sigma_{w}\right\}_{w \in \operatorname{pred}(v)}$ (that is, applicable given the sequences of values $\left\{\sigma_{w}\right\}_{w \in \operatorname{pred}(v)}$ respectively obtained by the parents of $v$ ). Let us now focus on $a_{1}^{\mathrm{w}}=a_{\mathrm{w}_{v} \mid \gamma}, a_{2}^{\mathrm{w}}=a_{\mathrm{w}_{v} \mid \mu}, a_{1}^{\mathrm{b}}=a_{\mathrm{b}_{v} \mid \delta}, a_{2}^{\mathrm{b}}=a_{\mathrm{b}_{v} \mid \nu}$ (that is, $\left.\{\gamma, \mu, \delta, \nu\} \subseteq \bigcup_{w \in \operatorname{pred}(v)}\left\{\mathrm{b}_{w}, \mathrm{w}_{w}\right\}\right) .{ }^{6}$

(I) If $\gamma=\delta \in\left\{\mathrm{b}_{w}, \mathrm{w}_{w}\right\}$, we set

$$
a_{i}^{*}= \begin{cases}a_{\mathrm{w}_{v} \mid \gamma} & i=2 j-1, j \in \mathbb{N} \\ a_{\mathrm{b}_{v} \mid \gamma} & \text { otherwise }\end{cases}
$$

In addition, we construct the following sets of ordering constraints. First, we set a binary relation $>_{v}$ over the action elements of $\rho_{v}^{*}=\left\langle a_{1}^{*}, \ldots, a_{k}^{*}\right\rangle$ to

$$
>_{v}=\left\{a_{i}^{*}<a_{j}^{*} \mid a_{i}^{*}, a_{j}^{*} \in \rho_{v}^{*}, i<j\right\} .
$$

It is immediate from Eq. 75 that $>_{v}$ is a strict total order over the elements of $\rho_{v}^{*}$. Likewise, if $\rho_{w}^{*}=\left\langle a_{j_{1}}, \ldots, a_{j_{l}}\right\rangle$, we set

$$
>_{v, w}= \begin{cases}\bigcup_{a_{i}^{*} \in \rho_{v}^{*}}\left\{a_{i}^{*}<a_{j_{1}}\right\}, & \gamma=\mathrm{b}_{w} \\ \bigcup_{a_{i}^{*} \in \rho_{v}^{*}}\left\{a_{i}^{*}>a_{j_{1}}\right\}, & \gamma=\mathrm{w}_{w}, l=1 \\ \bigcup_{a_{i}^{*} \in \rho_{v}^{*}}\left\{a_{i}^{*}>a_{j_{1}}\right\} \cup\left\{a_{i}^{*}<a_{j_{2}}\right\}, & \gamma=\mathrm{w}_{w}, l>1\end{cases}
$$

Finally, the ordering constraints $>_{v, w^{\prime}}$ for the rest of the parents $w^{\prime} \in \operatorname{pred}(v) \backslash\{w\}$ are set to empty sets.

For each $w \in \operatorname{pred}(v)$, it is easy to verify that the relation $>_{v, w}$ defined by Eq. 76 is a strict total order over its domain. Also, from Eqs. 75 and 76, we have that, for each $w \in \operatorname{pred}(v),>_{v} \cup>_{v, w}$ is a strict total order over the union of the elements of $\rho_{v}^{*}$ and $\rho_{w}^{*}$.

From Eqs. 75-76 we can now derive that any linearization of $>_{v} \cup \bigcup_{w \in \operatorname{pred}(v)}>_{v, w}$ defines a sequence of actions that is applicable with respect to $\{v\} \cup \operatorname{pred}(v)$. In addition, $\left|\rho_{v}^{*}\right|=\left|\rho \downarrow_{v}\right|$ together with Eq. 74 implies that this action sequence provides to $v$ the value $G[v]$ if the latter is specified.

6. It is possible that some of these actions do not exist, and our case by case analysis in the proof transparently takes this possibility into account. Specifically, if $a_{1}^{w}$ does not exist, then variable $v$ simply unchangeable, meaning $\rho \downarrow_{v}=\emptyset$. Next, if $a_{1}^{\mathrm{b}}$ does not exist, then $v$ can be changed at most once (from $\mathrm{b}$ to $\mathrm{w})$, and this is covered by a subcase of $(I)$. If $a_{2}^{\mathrm{w}}$ does not exist, and $a_{2}^{\mathrm{b}}$ does exist, then only sub-cases (a) of the cases $\{(I I I),(I V)\} .\{1,3,5,7\}$ are possible. Similarly, if $a_{2}^{\mathrm{b}}$ does not exist, and $a_{2}^{\mathrm{w}}$ does exist, then only sub-cases (b) of the cases $\{(I I I),(I V)\} \cdot\{1,3,5,7\}$ are possible. Finally, if both $a_{2}^{\mathrm{w}}$ and $a_{2}^{\mathrm{b}}$ do not exist, then cases $\{(I I I),(I V)\} \cdot\{1,3,5,7\}$ are not possible at all. 
(II) If $\gamma \in\left\{\mathrm{b}_{w}, \mathrm{w}_{w}\right\}$ and $\delta \in\left\{\mathrm{b}_{u}, \mathrm{w}_{u}\right\}$, such that $w \neq u$, we set

$$
a_{i}^{*}= \begin{cases}a_{\mathrm{w}_{v} \mid \gamma} & i=2 j-1, j \in \mathbb{N} \\ a_{\mathrm{b}_{v} \mid \delta} & \text { otherwise }\end{cases}
$$

In this case as well, the ordering constraints $>_{v}$ are set according to Eq. 75. Likewise, if $\rho_{w}^{*}=\left\langle a_{1}, \ldots, a_{l}\right\rangle$, and $\rho_{u}^{*}=\left\langle a_{1}^{\prime}, \ldots, a_{l^{\prime}}^{\prime}\right\rangle$, we set $\rangle_{v, w}$ according to Eq. 76 above, and $>_{v, u}$ according to Eq. 78 below.

$$
>_{v, u}= \begin{cases}\bigcup_{a_{i}^{*} \in \rho_{v}^{*}}\left\{a_{i}^{*}<a_{1}^{\prime}\right\}, & \nu=\mathrm{b}_{u} \\ \bigcup_{a_{i}^{*} \in \rho_{v}^{*}}\left\{a_{i}^{*}>a_{1}^{\prime}\right\}, & \nu=\mathrm{w}_{u}, l^{\prime}=1 \\ \bigcup_{a_{i}^{*} \in \rho_{v}^{*}}\left\{a_{i}^{*}>a_{1}^{\prime}\right\} \cup\left\{a_{i}^{*}<a_{2}^{\prime}\right\}, & \nu=\mathrm{w}_{u}, l^{\prime}>1\end{cases}
$$

Finally, the ordering constraints $>_{v, w^{\prime}}$ for the rest of the parents $w^{\prime} \in \operatorname{pred}(v) \backslash\{u, w\}$ are set to empty sets.

The relations $>_{v}$ in this case are identical to these in the previous case, and relations $>_{v, u}$ and $>_{v, w}$ are effectively identical to the relation $>_{v, w}$ in previous case. Thus, we have $>_{v} \cup>_{v, u}$ and $>_{v} \cup>_{v, w}$ forming strict partial orders over the unions of the elements of $\rho_{v}^{*}$ and $\rho_{u}^{*}$, and $\rho_{v}^{*}$ and $\rho_{w}^{*}$, respectively.

From Eqs. 75, 76, 78 we can now derive that any linearization of $>_{v} \cup \bigcup_{w \in \operatorname{pred}(v)}>_{v, w}$ defines a sequence of actions that is applicable with respect to $\{v\} \cup \operatorname{pred}(v)$. In addition, $\left|\rho_{v}^{*}\right|=\left|\rho \downarrow_{v}\right|$ together with Eq. 77 implies that this action sequence provides to $v$ the value $G[v]$ if the latter is specified.

(III) If $\gamma=\mathrm{b}_{w}, \delta=\mathrm{w}_{w}$, we distinguish between a few cases based on $\sigma_{w}$ and $\sigma_{v}$.

(1) If $\left|\rho \downarrow_{v}\right|=2 y+1,\left|\sigma_{w}\right|=2 x,\left|\sigma_{w}\right| \leq\left|\rho \downarrow_{v}\right|$, then we construct two post-3/2 candidates for $\rho_{v}^{*}$, and then assign $\rho_{v}^{*}$ to the cheapest among the two, proving that its cost has to be lower than $\mathcal{C}\left(\rho \downarrow_{v}\right)$.

(a) All the changes of $v$ to $\mathrm{w}_{v}$ are done using action $a_{1}^{\mathrm{w}}$, and then the largest possible number of changes to $\mathrm{b}_{v}$ are done using action $a_{1}^{\mathrm{b}}$, with the remaining changes to $\mathrm{b}_{v}$ being done using action $a_{2}^{\mathrm{b}}$. For this candidate for $\rho_{v}^{*}$, we set

$$
a_{i}^{*}= \begin{cases}a_{1}^{\mathrm{b}} & i=2 j, j \in \mathbb{N}, j<x \\ a_{2}^{\mathrm{b}} & i=2 j, j \in \mathbb{N}, x \leq j \leq y \\ a_{1}^{\mathrm{w}} & \text { otherwise }\end{cases}
$$

And the cost in this case is

$$
(y+1) \cdot \mathcal{C}\left(a_{1}^{\mathrm{w}}\right)+(x-1) \cdot \mathcal{C}\left(a_{1}^{\mathrm{b}}\right)+(y-x+1) \cdot \mathcal{C}\left(a_{2}^{\mathrm{b}}\right)
$$

The ordering constraints $>_{v}$ are set according to Eq. 75. Likewise, if $\rho_{w}^{*}=$ $\left\langle a_{1}, \ldots, a_{2 x-1}\right\rangle$, and $\rho_{u}^{*}=\left\langle a_{1}^{\prime}, \ldots, a_{l^{\prime}}^{\prime}\right\rangle$, we set $\rangle_{v, w}$ according to Eq. 81, and $>_{v, u}$ according to Eq. 82 .

$>_{v, w}=\bigcup_{a_{i}^{*} \in \rho_{v}^{*}, a_{j} \in \rho_{w}^{*}}\left\{a_{i}^{*}<a_{j} \mid i \leq j<2 x-1\right\} \cup\left\{a_{i}^{*}<a_{2 x-1}\right\} \cup\left\{a_{j}<a_{i}^{*} \mid j<i, j<2 x-1\right\}$ 
For each $u \in \operatorname{pred}(v) \backslash\{w\}$ we set,

$$
>_{v, u}=\left\{\begin{array}{ll}
\bigcup_{a_{i}^{*} \in \rho_{v}^{*}}\left\{a_{i}^{*}<a_{1}^{\prime}\right\}, & \nu=\mathrm{b}_{u} \\
\bigcup_{a_{i}^{*} \in \rho_{v}^{*}}\left\{a_{i}^{*}>a_{1}^{\prime}\right\}, & \nu=\mathrm{w}_{u}, l^{\prime}=1 \\
\bigcup_{a_{i}^{*} \in \rho_{v}^{*}}\left\{a_{i}^{*}>a_{1}^{\prime}\right\} \cup\left\{a_{i}^{*}<a_{2}^{\prime}\right\}, & \nu=\mathrm{w}_{u}, l^{\prime}>1 \\
\emptyset, & \text { otherwise }
\end{array} .\right.
$$

It is not hard to verify that the relation $>_{v, w}$ defined by Eq. 81 is a strict total order over its domain. Suppose to the contrary that for some $i, j$, both $a_{j}<a_{i}^{*}$ and $a_{i}^{*}<a_{j}$. Then from first inequality we have either $i \leq j<2 x-1$ or $j=2 x-1$, and from second we have $j<i, j<2 x-1$.

The relations $>_{v}$ and $>_{v, u}$ are effectively identical to these in case (II). Thus, we have $>_{v} \cup>_{v, u}$ and $>_{v} \cup>_{v, w}$ forming strict partial orders over the unions of the elements of $\rho_{v}^{*}$ and $\rho_{u}^{*}$, and $\rho_{v}^{*}$ and $\rho_{w}^{*}$, respectively.

From Eqs. 75, 81, 82 we can now derive that any linearization of $>_{v} \cup \bigcup_{w \in \operatorname{pred}(v)}>_{v, w}$ defines a sequence of actions that is applicable with respect to $\{v\} \cup \operatorname{pred}(v)$. In addition, $\left|\rho_{v}^{*}\right|=\left|\rho \downarrow_{v}\right|$ together with Eq. 79 implies that this action sequence provides to $v$ the value $G[v]$ if the latter is specified.

(b) All the changes of $v$ to $\mathrm{b}_{v}$ are done using action $a_{1}^{\mathrm{b}}$, and then the largest possible number of changes to $\mathrm{w}_{v}$ are done using action $a_{1}^{\mathrm{w}}$, with the remaining changes to $\mathrm{w}_{v}$ being done using action $a_{2}^{\mathrm{w}}$. For this candidate for $\rho_{v}^{*}$, we set

$$
a_{i}^{*}= \begin{cases}a_{1}^{\mathrm{w}} & i=2 j-1, j \in \mathbb{N}, j \leq x \\ a_{2}^{\mathrm{w}} & i=2 j-1, j \in \mathbb{N}, x<j \leq y+1 \\ a_{1}^{\mathrm{b}} & \text { otherwise }\end{cases}
$$

And the cost in this case is

$$
x \cdot \mathcal{C}\left(a_{1}^{\mathrm{w}}\right)+(y+1-x) \cdot \mathcal{C}\left(a_{2}^{\mathrm{w}}\right)+y \cdot \mathcal{C}\left(a_{1}^{\mathrm{b}}\right)
$$

The ordering constraints $>_{v}$ are set according to Eq. 75. Likewise, if $\rho_{w}^{*}=$ $\left\langle a_{1}, \ldots, a_{2 x-1}\right\rangle$, and $\rho_{u}^{*}=\left\langle a_{1}^{\prime}, \ldots, a_{l^{\prime}}^{\prime}\right\rangle$, we set $\rangle_{v, w}$ according to Eq. 85, and $>_{v, u}$ according to Eq. 86 .

$$
>_{v, w}=\bigcup_{a_{i}^{*} \in \rho_{v}^{*}, a_{j} \in \rho_{w}^{*}}\left\{a_{i}^{*}<a_{j} \mid i \leq j\right\} \cup\left\{a_{j}<a_{i}^{*} \mid j<i\right\}
$$

For each $u \in \operatorname{pred}(v) \backslash\{w\}$ we set,

$$
>_{v, u}=\left\{\begin{array}{ll}
\bigcup_{a_{i}^{*} \in \rho_{v}^{*}}\left\{a_{i}^{*}<a_{1}^{\prime}\right\}, & \mu=\mathrm{b}_{u} \\
\bigcup_{a_{i}^{*} \in \rho_{v}^{*}}\left\{a_{i}^{*}>a_{1}^{\prime}\right\}, & \mu=\mathrm{w}_{u}, l^{\prime}=1 \\
\bigcup_{a_{i}^{*} \in \rho_{v}^{*}}\left\{a_{i}^{*}>a_{1}^{\prime}\right\} \cup\left\{a_{i}^{*}<a_{2}^{\prime}\right\}, & \mu=\mathrm{w}_{u}, l^{\prime}>1 \\
\emptyset, & \text { otherwise }
\end{array} .\right.
$$

The relation $>_{v, w}$ defined by Eq. 85 is a strict total order over its domain. The relations $>_{v}$ and $>_{v, u}$ are effectively identical to these in case (II). Thus, 
we have $>_{v} \cup>_{v, u}$ and $>_{v} \cup>_{v, w}$ forming strict partial orders over the unions of the elements of $\rho_{v}^{*}$ and $\rho_{u}^{*}$, and $\rho_{v}^{*}$ and $\rho_{w}^{*}$, respectively.

From Eqs. 75, 85, 86 we can now derive that any linearization of $>_{v} \cup \bigcup_{w \in \operatorname{pred}(v)}>_{v, w}$ defines a sequence of actions that is applicable with respect to $\{v\} \cup \operatorname{pred}(v)$. In addition, $\left|\rho_{v}^{*}\right|=\left|\rho \downarrow_{v}\right|$ together with Eq. 83 implies that this action sequence provides to $v$ the value $G[v]$ if the latter is specified.

Now, for each cost-optimal plan $\rho, \rho \downarrow_{v}$ cannot contain more than $y+x$ actions of both types $a_{1}^{w}$ and $a_{1}^{\text {b }}$ totally. Suppose to the contrary that $\rho \downarrow_{v}$ contain at least $y+x+1$ actions of types $a_{1}^{\mathrm{w}}$ and $a_{1}^{\mathrm{b}}$. Then it contains no more than $y-x$ actions of other types. Let $\mathrm{b}_{w} \cdot \mathrm{w}_{w} \cdot \ldots \cdot \mathrm{b}_{w}$ sequence of $2 y+1$ values of $w$ that support a cost-optimal plan for $v$ given that $w$ can change its value any number of times. Then each action of any other type will decrease the needed length of this sequence by at most 2 . Therefore at most $y-x$ actions of other type will decrease the length by at most $2 y-2 x$, and we are left with the sequence of length $\geq 2 y+1-(2 y-2 x)=2 x+1$. Therefore $\sigma_{w}$ cannot support more than $y+x$ actions of types $a_{1}^{\mathrm{w}}$ and $a_{1}^{\mathrm{b}}$. Now, suppose that in some given cost-optimal plan $\rho \downarrow_{v}$ for $v$ there are $\alpha$ actions of type $a_{1}^{\mathrm{w}}$ and $\beta$ actions of type $a_{1}^{\mathrm{b}}$. Then

$$
\alpha+\beta \leq y+x
$$

and

$$
\mathcal{C}\left(\rho \downarrow_{v}\right) \geq \alpha \cdot \mathcal{C}\left(a_{1}^{\mathrm{w}}\right)+(y+1-\alpha) \cdot \mathcal{C}\left(a_{2}^{\mathrm{w}}\right)+\beta \cdot \mathcal{C}\left(a_{1}^{\mathrm{b}}\right)+(y-\beta) \cdot \mathcal{C}\left(a_{2}^{\mathrm{b}}\right)
$$

For $(80) \leq(84)$, we have

$$
\mathcal{C}\left(a_{2}^{\mathrm{b}}\right)-\mathcal{C}\left(a_{1}^{\mathrm{b}}\right) \leq \mathcal{C}\left(a_{2}^{\mathrm{w}}\right)-\mathcal{C}\left(a_{1}^{\mathrm{w}}\right)
$$

Now suppose to the contrary that the plan in first case is not cost-optimal. Then from Eq. 88 we have

$$
\begin{aligned}
& \alpha \cdot \mathcal{C}\left(a_{1}^{\mathrm{w}}\right)+(y+1-\alpha) \cdot \mathcal{C}\left(a_{2}^{\mathrm{w}}\right)+\beta \cdot \mathcal{C}\left(a_{1}^{\mathrm{b}}\right)+(y-\beta) \cdot \mathcal{C}\left(a_{2}^{\mathrm{b}}\right)< \\
& (y+1) \cdot \mathcal{C}\left(a_{1}^{\mathrm{w}}\right)+(x-1) \cdot \mathcal{C}\left(a_{1}^{\mathrm{b}}\right)+(y-x+1) \cdot \mathcal{C}\left(a_{2}^{\mathrm{b}}\right)
\end{aligned}
$$

and from it

$$
(y+1-\alpha) \cdot\left(\mathcal{C}\left(a_{2}^{\mathrm{w}}\right)-\mathcal{C}\left(a_{1}^{\mathrm{w}}\right)\right)<(\beta-x+1) \cdot\left(\mathcal{C}\left(a_{2}^{\mathrm{b}}\right)-\mathcal{C}\left(a_{1}^{\mathrm{b}}\right)\right)
$$

From Eq. 87 we have $y+1-\alpha \geq \beta-x+1$, together with Eq. 89 contradicting Eq. 90.

For $(84) \leq(80)$, we have

$$
\mathcal{C}\left(a_{2}^{\mathrm{w}}\right)-\mathcal{C}\left(a_{1}^{\mathrm{w}}\right) \leq \mathcal{C}\left(a_{2}^{\mathrm{b}}\right)-\mathcal{C}\left(a_{1}^{\mathrm{b}}\right)
$$

Now suppose to the contrary that the plan in second case is not cost-optimal. Then from Eq. 88 we have

$$
\begin{aligned}
& \alpha \cdot \mathcal{C}\left(a_{1}^{\mathrm{w}}\right)+(y+1-\alpha) \cdot \mathcal{C}\left(a_{2}^{\mathrm{w}}\right)+\beta \cdot \mathcal{C}\left(a_{1}^{\mathrm{b}}\right)+(y-\beta) \cdot \mathcal{C}\left(a_{2}^{\mathrm{b}}\right)< \\
& x \cdot \mathcal{C}\left(a_{1}^{\mathrm{w}}\right)+(y-x+1) \cdot \mathcal{C}\left(a_{2}^{\mathrm{w}}\right)+y \cdot \mathcal{C}\left(a_{1}^{\mathrm{b}}\right)
\end{aligned}
$$


and from it

$$
(y-\beta) \cdot\left(\mathcal{C}\left(a_{2}^{\mathrm{b}}\right)-\mathcal{C}\left(a_{1}^{\mathrm{b}}\right)\right)<(\alpha-x) \cdot\left(\mathcal{C}\left(a_{2}^{\mathrm{w}}\right)-\mathcal{C}\left(a_{1}^{\mathrm{w}}\right)\right)
$$

From Eq. 87 we have $y-\beta \geq \alpha-x$, together with Eq. 91 contradicting Eq. 92 . (2) If $\left|\rho \downarrow_{v}\right|=2 y+1,\left|\sigma_{w}\right|=2 x,\left|\sigma_{w}\right|>\left|\rho \downarrow_{v}\right|$, then the actions of $\rho_{v}^{*}$ are set to

$$
a_{i}^{*}= \begin{cases}a_{\mathrm{w}_{v} \mid \mathrm{b}_{w}} & i=2 j-1, j \in \mathbb{N} \\ a_{\mathrm{b}_{v} \mid \mathrm{w}_{w}} & \text { otherwise }\end{cases}
$$

In this case as well, the ordering constraints $>_{v}$ are set according to Eq. 75 . Likewise, if $\rho_{w}^{*}=\left\langle a_{1}, \ldots, a_{2 x-1}\right\rangle$, we set $\rangle_{v, w}$ according to Eq. 85 above. Finally, the ordering constraints $>_{v, w^{\prime}}$ for the rest of the parents $w^{\prime} \in \operatorname{pred}(v) \backslash\{u, w\}$ are set to empty sets.

The relations $>_{v}$ and $>_{v, w}$ are identical to the previous case. Thus, we have $>_{v} \cup>_{v, w}$ forming strict partial order over the union of the elements of $\rho_{v}^{*}$ and $\rho_{w}^{*}$.

From Eqs. 75, 85 we can now derive that any linearization of $>_{v} \cup \bigcup_{w \in \operatorname{pred}(v)}>_{v, w}$ defines a sequence of actions that is applicable with respect to $\{v\} \cup \operatorname{pred}(v)$. In addition, $\left|\rho_{v}^{*}\right|=\left|\rho \downarrow_{v}\right|$ together with Eq. 93 implies that this action sequence provides to $v$ the value $G[v]$ if the latter is specified.

(3) If $\left|\rho \downarrow_{v}\right|=2 y,\left|\sigma_{w}\right|=2 x,\left|\sigma_{w}\right|<\left|\rho \downarrow_{v}\right|$, then we construct two post-3/2 candidates for $\rho_{v}^{*}$, and then assign $\rho_{v}^{*}$ to the cheapest among the two, proving that its cost has to be lower than $\mathcal{C}\left(\rho \downarrow_{v}\right)$.

(a) All the changes of $v$ to $\mathrm{w}_{v}$ are done using action $a_{1}^{\mathrm{w}}$, and then the largest possible number of changes to $\mathrm{b}_{v}$ are done using action $a_{1}^{\mathrm{b}}$, with the remaining changes to $\mathrm{b}_{v}$ being done using action $\mathrm{a}_{2}^{\mathrm{b}}$. For this candidate for $\rho_{v}^{*}$, we set

$$
a_{i}^{*}= \begin{cases}a_{2}^{\mathrm{b}} & i=2 j, j \in \mathbb{N}, j \leq y-x \\ a_{1}^{\mathrm{b}} & i=2 j, j \in \mathbb{N}, y-x<j \leq y \\ a_{1}^{\mathrm{w}} & \text { otherwise }\end{cases}
$$

And the cost in this case is

$$
y \cdot \mathcal{C}\left(a_{1}^{\mathrm{w}}\right)+x \cdot \mathcal{C}\left(a_{1}^{\mathrm{b}}\right)+(y-x) \cdot \mathcal{C}\left(a_{2}^{\mathrm{b}}\right)
$$

The ordering constraints $>_{v}$ are set according to Eq. 75. Likewise, if $\rho_{w}^{*}=$ $\left\langle a_{1}, \ldots, a_{2 x-1}\right\rangle$, we set $>_{v, w}$ according to Eq. 96 .

$$
>_{v, w}=\bigcup_{a_{i}^{*} \in \rho_{v}^{*}, a_{j} \in \rho_{w}^{*}}\left\{a_{i}^{*}<a_{j} \mid i \leq 2 y-2 x+j\right\} \cup\left\{a_{j}<a_{i}^{*} \mid i>2 y-2 x+j\right\}
$$

For each $u \in \operatorname{pred}(v) \backslash\{w\}$ we set $>_{v, u}$ according to Eq. 82. It is easy to verify that the relation $>_{v, w}$ defined by Eq. 96 is a strict total order over its domain. The relations $>_{v}$ and $>_{v, u}$ are effectively identical to the previous 
case. Thus, we have $>_{v} \cup>_{v, u}$ and $>_{v} \cup>_{v, w}$ forming strict partial orders over the unions of the elements of $\rho_{v}^{*}$ and $\rho_{u}^{*}$, and $\rho_{v}^{*}$ and $\rho_{w}^{*}$, respectively. From Eqs. 75, 82, 96 we can now derive that any linearization of $>_{v} \cup \bigcup_{w \in \operatorname{pred}(v)}>_{v, w}$ defines a sequence of actions that is applicable with respect to $\{v\} \cup \operatorname{pred}(v)$. In addition, $\left|\rho_{v}^{*}\right|=\left|\rho \downarrow_{v}\right|$ together with Eq. 94 implies that this action sequence provides to $v$ the value $G[v]$ if the latter is specified.

(b) All the changes of $v$ to $\mathrm{b}_{v}$ are done using action $a_{1}^{\mathrm{b}}$, and then the largest possible number of changes to $\mathrm{w}_{v}$ are done using action $a_{1}^{\mathrm{w}}$, with the remaining changes to $\mathrm{w}_{v}$ being done using action $a_{2}^{\mathrm{w}}$. For this candidate for $\rho_{v}^{*}$, we set

$$
a_{i}^{*}= \begin{cases}a_{1}^{\mathrm{w}} & i=2 j-1, j \in \mathbb{N}, j \leq x \\ a_{2}^{\mathrm{w}} & i=2 j-1, j \in \mathbb{N}, x<j \leq y \\ a_{1}^{\mathrm{b}} & \text { otherwise }\end{cases}
$$

And the cost in this case is

$$
x \cdot \mathcal{C}\left(a_{1}^{\mathrm{w}}\right)+(y-x) \cdot \mathcal{C}\left(a_{2}^{\mathrm{w}}\right)+y \cdot \mathcal{C}\left(a_{1}^{\mathrm{b}}\right)
$$

The ordering constraints $>_{v}$ are set according to Eq. 75. Likewise, if $\rho_{w}^{*}=$ $\left\langle a_{1}, \ldots, a_{2 x-1}\right\rangle$, we set $>_{v, w}$ according to Eq. 85 above.

For each $u \in \operatorname{pred}(v) \backslash\{w\}$ we set $>_{v, u}$ according to Eq. 86. The relations $>_{v}$, $>_{v, w}$ and $>_{v, u}$ are effectively identical to the previous case. Thus, again, we have $>_{v} \cup>_{v, u}$ and $>_{v} \cup>_{v, w}$ forming strict partial orders over the unions of the elements of $\rho_{v}^{*}$ and $\rho_{u}^{*}$, and $\rho_{v}^{*}$ and $\rho_{w}^{*}$, respectively.

From Eqs. 75, 85, 86 we can now derive that any linearization of $>_{v} \cup \bigcup_{w \in \operatorname{pred}(v)}>_{v, w}$ defines a sequence of actions that is applicable with respect to $\{v\} \cup \operatorname{pred}(v)$. In addition, $\left|\rho_{v}^{*}\right|=\left|\rho \downarrow_{v}\right|$ together with Eq. 97 implies that this action sequence provides to $v$ the value $G[v]$ if the latter is specified.

Now, for each cost-optimal plan $\rho, \rho \downarrow_{v}$ cannot contain more than $y+x$ actions of both types $a_{1}^{\mathrm{w}}$ and $a_{1}^{\mathrm{b}}$ totally. Suppose to the contrary that $\rho \downarrow_{v}$ contain at least $y+x+1$ actions of types $a_{1}^{\mathrm{w}}$ and $a_{1}^{\mathrm{b}}$. Then it contains no more than $y-x-1$ actions of other types. Let $\mathrm{b}_{w} \cdot \mathrm{w}_{w} \cdot \ldots \cdot \mathrm{w}_{w}$ sequence of $2 y$ values of $w$ that support a cost-optimal plan for $v$ given that $w$ can change its value any number of times. Then each action of any other type will decrease the needed length of this sequence by at most 2 . Therefore at most $y-x-1$ actions of other type will decrease the length by at most $2 y-2 x-2$, and we are left with the sequence of length $\geq 2 y-(2 y-2 x-2)=2 x+2$. Therefore $\sigma_{w}$ cannot support more than $y+x$ actions of types $a_{1}^{\mathrm{w}}$ and $a_{1}^{\mathrm{b}}$. Now, suppose that in some given cost-optimal plan $\rho \downarrow_{v}$ for $v$ there are $\alpha$ actions of type $a_{1}^{\mathrm{w}}$ and $\beta$ actions of type $a_{1}^{\mathrm{b}}$. Then

$$
\alpha+\beta \leq y+x
$$

and

$$
\mathcal{C}\left(\rho \downarrow_{v}\right) \geq \alpha \cdot \mathcal{C}\left(a_{1}^{\mathrm{w}}\right)+(y-\alpha) \cdot \mathcal{C}\left(a_{2}^{\mathrm{w}}\right)+\beta \cdot \mathcal{C}\left(a_{1}^{\mathrm{b}}\right)+(y-\beta) \cdot \mathcal{C}\left(a_{2}^{\mathrm{b}}\right)
$$


For $(95) \leq(98)$, we have

$$
\mathcal{C}\left(a_{2}^{\mathrm{b}}\right)-\mathcal{C}\left(a_{1}^{\mathrm{b}}\right) \leq \mathcal{C}\left(a_{2}^{\mathrm{w}}\right)-\mathcal{C}\left(a_{1}^{\mathrm{w}}\right)
$$

Now suppose to the contrary that the plan in first case is not cost-optimal. Then from Eq. 100 we have

$$
\begin{aligned}
& \alpha \cdot \mathcal{C}\left(a_{1}^{\mathrm{w}}\right)+(y-\alpha) \cdot \mathcal{C}\left(a_{2}^{\mathrm{w}}\right)+\beta \cdot \mathcal{C}\left(a_{1}^{\mathrm{b}}\right)+(y-\beta) \cdot \mathcal{C}\left(a_{2}^{\mathrm{b}}\right)< \\
& y \cdot \mathcal{C}\left(a_{1}^{\mathrm{w}}\right)+x \cdot \mathcal{C}\left(a_{1}^{\mathrm{b}}\right)+(y-x) \cdot \mathcal{C}\left(a_{2}^{\mathrm{b}}\right)
\end{aligned}
$$

and from it

$$
(y-\alpha) \cdot\left(\mathcal{C}\left(a_{2}^{\mathrm{w}}\right)-\mathcal{C}\left(a_{1}^{\mathrm{w}}\right)\right)<(\beta-x) \cdot\left(\mathcal{C}\left(a_{2}^{\mathrm{b}}\right)-\mathcal{C}\left(a_{1}^{\mathrm{b}}\right)\right)
$$

From Eq. 99 we have $y-\alpha \geq \beta-x$, together with Eq. 101 contradicting Eq. 102. For $(98) \leq(95)$, we have

$$
\mathcal{C}\left(a_{2}^{\mathrm{w}}\right)-\mathcal{C}\left(a_{1}^{\mathrm{w}}\right) \leq \mathcal{C}\left(a_{2}^{\mathrm{b}}\right)-\mathcal{C}\left(a_{1}^{\mathrm{b}}\right)
$$

Now suppose to the contrary that the plan in second case is not cost-optimal. Then from Eq. 100 we have

$$
\begin{aligned}
& \alpha \cdot \mathcal{C}\left(a_{1}^{\mathrm{w}}\right)+(y-\alpha) \cdot \mathcal{C}\left(a_{2}^{\mathrm{w}}\right)+\beta \cdot \mathcal{C}\left(a_{1}^{\mathrm{b}}\right)+(y-\beta) \cdot \mathcal{C}\left(a_{2}^{\mathrm{b}}\right)< \\
& x \cdot \mathcal{C}\left(a_{1}^{\mathrm{w}}\right)+(y-x) \cdot \mathcal{C}\left(a_{2}^{\mathrm{w}}\right)+y \cdot \mathcal{C}\left(a_{1}^{\mathrm{b}}\right)
\end{aligned}
$$

and from it

$$
(y-\beta) \cdot\left(\mathcal{C}\left(a_{2}^{\mathrm{b}}\right)-\mathcal{C}\left(a_{1}^{\mathrm{b}}\right)\right)<(\alpha-x) \cdot\left(\mathcal{C}\left(a_{2}^{\mathrm{w}}\right)-\mathcal{C}\left(a_{1}^{\mathrm{w}}\right)\right)
$$

From Eq. 99 we have $y-\beta \geq \alpha-x$, together with Eq. 103 contradicting Eq. 104 .

(4) If $\left|\rho \downarrow_{v}\right|=2 y,\left|\sigma_{w}\right|=2 x,\left|\sigma_{w}\right| \geq\left|\rho \downarrow_{v}\right|$, then the actions of $\rho_{v}^{*}$ are set to

$$
a_{i}^{*}= \begin{cases}a_{\mathrm{w}_{v} \mid \mathrm{b}_{w}} & i=2 j-1, j \in \mathbb{N} \\ a_{\mathrm{b}_{v} \mid \mathrm{w}_{w}} & \text { otherwise }\end{cases}
$$

The ordering constraints $>_{v}$ are set according to Eq. 75. Likewise, if $\rho_{w}^{*}=$ $\left\langle a_{1}, \ldots, a_{2 x-1}\right\rangle$, we set $>_{v, w}$ according to Eq. 85 above. Finally, the ordering constraints $>_{v, w^{\prime}}$ for the rest of the parents $w^{\prime} \in \operatorname{pred}(v) \backslash\{u, w\}$ are set to empty sets. The relations $>_{v}$ and $>_{v, w}$ are identical to the previous case. Thus, we have $>_{v} \cup>_{v, w}$ forming strict partial order over the union of the elements of $\rho_{v}^{*}$ and $\rho_{w}^{*}$.

From Eqs. 75, 85 we can now derive that any linearization of $>_{v} \cup \bigcup_{w \in \operatorname{pred}(v)}>_{v, w}$ defines a sequence of actions that is applicable with respect to $\{v\} \cup \operatorname{pred}(v)$. In addition, $\left|\rho_{v}^{*}\right|=\left|\rho \downarrow_{v}\right|$ together with Eq. 105 implies that this action sequence provides to $v$ the value $G[v]$ if the latter is specified.

(5) If $\left|\rho \downarrow_{v}\right|=2 y+1,\left|\sigma_{w}\right|=2 x+1,\left|\sigma_{w}\right|<\left|\rho \downarrow_{v}\right|$, then we construct two post-3/2 candidates for $\rho_{v}^{*}$, and then assign $\rho_{v}^{*}$ to the cheapest among the two, proving that its cost has to be lower than $\mathcal{C}\left(\rho \downarrow_{v}\right)$. 
(a) All the changes of $v$ to $\mathrm{w}_{v}$ are done using action $a_{1}^{\mathrm{w}}$, and then the largest possible number of changes to $\mathrm{b}_{v}$ are done using action $a_{1}^{b}$, with the remaining changes to $\mathrm{b}_{v}$ being done using action $a_{2}^{\mathrm{b}}$. For this candidate for $\rho_{v}^{*}$, we set

$$
a_{i}^{*}= \begin{cases}a_{2}^{\mathrm{b}} & i=2 j, j \in \mathbb{N}, j \leq y-x \\ a_{1}^{\mathrm{b}} & i=2 j, j \in \mathbb{N}, y-x<j \leq y \\ a_{1}^{\mathrm{w}} & \text { otherwise }\end{cases}
$$

And the cost in this case is

$$
(y+1) \cdot \mathcal{C}\left(a_{1}^{\mathrm{w}}\right)+x \cdot \mathcal{C}\left(a_{1}^{\mathrm{b}}\right)+(y-x) \cdot \mathcal{C}\left(a_{2}^{\mathrm{b}}\right)
$$

The ordering constraints $>_{v}$ are set according to Eq. 75. Likewise, if $\rho_{w}^{*}=$ $\left\langle a_{1}, \ldots, a_{2 x}\right\rangle$, we set $>_{v, w}$ according to Eq. 96 above. For each $u \in \operatorname{pred}(v) \backslash$ $\{w\}$ we set $>_{v, u}$ according to Eq. 82 above. The relations $>_{v},>_{v, w}$ and $>_{v, u}$ are effectively identical to the previous case. Thus, we have $>_{v} \cup>_{v, u}$ and $>_{v} \cup>_{v, w}$ forming strict partial orders over the unions of the elements of $\rho_{v}^{*}$ and $\rho_{u}^{*}$, and $\rho_{v}^{*}$ and $\rho_{w}^{*}$, respectively.

From Eqs. 75, 82, 96 we can now derive that any linearization of $>_{v} \cup \bigcup_{w \in \operatorname{pred}(v)}>_{v, w}$ defines a sequence of actions that is applicable with respect to $\{v\} \cup \operatorname{pred}(v)$. In addition, $\left|\rho_{v}^{*}\right|=\left|\rho \downarrow_{v}\right|$ together with Eq. 106 implies that this action sequence provides to $v$ the value $G[v]$ if the latter is specified.

(b) All the changes of $v$ to $\mathrm{b}_{v}$ are done using action $a_{1}^{\mathrm{b}}$, and then the largest possible number of changes to $\mathrm{w}_{v}$ are done using action $a_{1}^{\mathrm{w}}$, with the remaining changes to $\mathrm{w}_{v}$ being done using action $a_{2}^{\mathrm{w}}$. For this candidate for $\rho_{v}^{*}$, we set

$$
a_{i}^{*}= \begin{cases}a_{1}^{\mathrm{w}} & i=2 j-1, j \in \mathbb{N}, j \leq x \text { or } j=y+1 \\ a_{2}^{\mathrm{w}} & i=2 j-1, j \in \mathbb{N}, x<j \leq y \\ a_{1}^{\mathrm{b}} & \text { otherwise }\end{cases}
$$

And the cost in this case is

$$
(x+1) \cdot \mathcal{C}\left(a_{1}^{\mathrm{w}}\right)+(y-x) \cdot \mathcal{C}\left(a_{2}^{\mathrm{w}}\right)+y \cdot \mathcal{C}\left(a_{1}^{\mathrm{b}}\right)
$$

The ordering constraints $>_{v}$ are set according to Eq. 75. Likewise, if $\rho_{w}^{*}=$ $\left\langle a_{1}, \ldots, a_{2 x}\right\rangle$, we set $\rangle_{v, w}$ according to Eq. 110 .

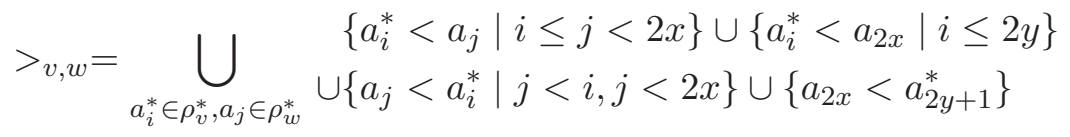

For each $u \in \operatorname{pred}(v) \backslash\{w\}$ we set $>_{v, u}$ according to Eq. 86 above.

It is easy to verify that the relation $>_{v, w}$ defined by Eq. 110 is a strict total order over its domain. The relations $>_{v}$ and $>_{v, u}$ are effectively identical to the previous case. Thus, we have $>_{v} \cup>_{v, u}$ and $>_{v} \cup>_{v, w}$ forming strict partial orders over the unions of the elements of $\rho_{v}^{*}$ and $\rho_{u}^{*}$, and $\rho_{v}^{*}$ and $\rho_{w}^{*}$, respectively. 
From Eqs. 75, 86, 110 we can now derive that any linearization of $>_{v} \cup \bigcup_{w \in \operatorname{pred}(v)}>_{v, w}$ defines a sequence of actions that is applicable with respect to $\{v\} \cup \operatorname{pred}(v)$. In addition, $\left|\rho_{v}^{*}\right|=\left|\rho \downarrow_{v}\right|$ together with Eq. 108 implies that this action sequence provides to $v$ the value $G[v]$ if the latter is specified.

Now, for each cost-optimal plan $\rho, \rho \downarrow_{v}$ cannot contain more than $y+x+1$ actions of both types $a_{1}^{w}$ and $a_{1}^{\mathrm{b}}$ totally. Suppose to the contrary that $\rho \downarrow_{v}$ contain at least $y+x+2$ actions of types $a_{1}^{\mathrm{w}}$ and $a_{1}^{\mathrm{b}}$. Then it contains no more than $y-x-1$ actions of other types. Let $\mathbf{b}_{w} \cdot \mathbf{w}_{w} \cdot \ldots \cdot \mathbf{b}_{w}$ sequence of $2 y+1$ values of $w$ that support a cost-optimal plan for $v$ given that $w$ can change its value any number of times. Then each action of any other type will decrease the needed length of this sequence by at most 2 . Therefore at most $y-x-1$ actions of other type will decrease the length by at most $2 y-2 x-2$, and we are left with the sequence of length $\geq 2 y+1-(2 y-2 x-2)=2 x+3$. Therefore $\sigma_{w}$ cannot support more than $y+x+1$ actions of types $a_{1}^{\mathrm{w}}$ and $a_{1}^{\mathrm{b}}$. Now, suppose that in some given cost-optimal plan $\rho \downarrow_{v}$ for $v$ there are $\alpha$ actions of type $a_{1}^{\mathrm{w}}$ and $\beta$ actions of type $a_{1}^{\text {b. }}$. Then

$$
\alpha+\beta \leq y+x+1
$$

and

$$
\mathcal{C}\left(\rho \downarrow_{v}\right) \geq \alpha \cdot \mathcal{C}\left(a_{1}^{\mathrm{w}}\right)+(y+1-\alpha) \cdot \mathcal{C}\left(a_{2}^{\mathrm{w}}\right)+\beta \cdot \mathcal{C}\left(a_{1}^{\mathrm{b}}\right)+(y-\beta) \cdot \mathcal{C}\left(a_{2}^{\mathrm{b}}\right)
$$

For $(107) \leq(109)$, we have

$$
\mathcal{C}\left(a_{2}^{\mathrm{b}}\right)-\mathcal{C}\left(a_{1}^{\mathrm{b}}\right) \leq \mathcal{C}\left(a_{2}^{\mathrm{w}}\right)-\mathcal{C}\left(a_{1}^{\mathrm{w}}\right)
$$

Now suppose to the contrary that the plan in first case is not cost-optimal. Then from Eq. 112 we have

$$
\begin{aligned}
& \alpha \cdot \mathcal{C}\left(a_{1}^{\mathrm{w}}\right)+(y+1-\alpha) \cdot \mathcal{C}\left(a_{2}^{\mathrm{w}}\right)+\beta \cdot \mathcal{C}\left(a_{1}^{\mathrm{b}}\right)+(y-\beta) \cdot \mathcal{C}\left(a_{2}^{\mathrm{b}}\right)< \\
& (y+1) \cdot \mathcal{C}\left(a_{1}^{\mathrm{w}}\right)+x \cdot \mathcal{C}\left(a_{1}^{\mathrm{b}}\right)+(y-x) \cdot \mathcal{C}\left(a_{2}^{\mathrm{b}}\right)
\end{aligned}
$$

and from it

$$
(y+1-\alpha) \cdot\left(\mathcal{C}\left(a_{2}^{\mathrm{w}}\right)-\mathcal{C}\left(a_{1}^{\mathrm{w}}\right)\right)<(\beta-x) \cdot\left(\mathcal{C}\left(a_{2}^{\mathrm{b}}\right)-\mathcal{C}\left(a_{1}^{\mathrm{b}}\right)\right)
$$

From Eq. 111 we have $y+1-\alpha \geq \beta-x$, together with Eq. 113 contradicting Eq. 114.

For $(109) \leq(107)$, we have

$$
\mathcal{C}\left(a_{2}^{\mathrm{w}}\right)-\mathcal{C}\left(a_{1}^{\mathrm{w}}\right) \leq \mathcal{C}\left(a_{2}^{\mathrm{b}}\right)-\mathcal{C}\left(a_{1}^{\mathrm{b}}\right)
$$

Now suppose to the contrary that the plan in second case is not cost-optimal. Then from Eq. 112 we have

$$
\begin{aligned}
& \alpha \cdot \mathcal{C}\left(a_{1}^{\mathrm{w}}\right)+(y+1-\alpha) \cdot \mathcal{C}\left(a_{2}^{\mathrm{w}}\right)+\beta \cdot \mathcal{C}\left(a_{1}^{\mathrm{b}}\right)+(y-\beta) \cdot \mathcal{C}\left(a_{2}^{\mathrm{b}}\right)< \\
& \left(x+1 \cdot \mathcal{C}\left(a_{1}^{\mathrm{w}}\right)+(y-x) \cdot \mathcal{C}\left(a_{2}^{\mathrm{w}}\right)+y \cdot \mathcal{C}\left(a_{1}^{\mathrm{b}}\right)\right.
\end{aligned}
$$


and from it

$$
(y-\beta) \cdot\left(\mathcal{C}\left(a_{2}^{\mathrm{b}}\right)-\mathcal{C}\left(a_{1}^{\mathrm{b}}\right)\right)<(\alpha-x-1) \cdot\left(\mathcal{C}\left(a_{2}^{\mathrm{w}}\right)-\mathcal{C}\left(a_{1}^{\mathrm{w}}\right)\right)
$$

From Eq. 111 we have $y-\beta \geq \alpha-x-1$, together with Eq. 115 contradicting Eq. 116.

(6) If $\left|\rho \downarrow_{v}\right|=2 y+1,\left|\sigma_{w}\right|=2 x+1,\left|\sigma_{w}\right| \geq\left|\rho \downarrow_{v}\right|$, the actions of $\rho_{v}^{*}$ are set to

$$
a_{i}^{*}= \begin{cases}a_{\mathrm{w}_{v} \mid \mathrm{b}_{w}} & i=2 j-1, j \in \mathbb{N} \\ a_{\mathrm{b}_{v} \mid \mathrm{w}_{w}} & \text { otherwise }\end{cases}
$$

The ordering constraints $>_{v}$ are set according to Eq. 75. Likewise, if $\rho_{w}^{*}=$ $\left\langle a_{1}, \ldots, a_{2 x}\right\rangle$, we set $>_{v, w}$ according to Eq. 85 above. Finally, the ordering constraints $>_{v, w^{\prime}}$ for the rest of the parents $w^{\prime} \in \operatorname{pred}(v) \backslash\{u, w\}$ are set to empty sets. The relations $>_{v}$ and $>_{v, w}$ are identical to the previous case. Thus, we have $>_{v} \cup>_{v, w}$ forming strict partial order over the union of the elements of $\rho_{v}^{*}$ and $\rho_{w}^{*}$.

From Eqs. 75, 85 we can now derive that any linearization of $>_{v} \cup \bigcup_{w \in \operatorname{pred}(v)}>_{v, w}$ defines a sequence of actions that is applicable with respect to $\{v\} \cup \operatorname{pred}(v)$. In addition, $\left|\rho_{v}^{*}\right|=\left|\rho \downarrow_{v}\right|$ together with Eq. 117 implies that this action sequence provides to $v$ the value $G[v]$ if the latter is specified.

(7) If $\left|\rho \downarrow_{v}\right|=2 y,\left|\sigma_{w}\right|=2 x+1,\left|\sigma_{w}\right| \leq\left|\rho \downarrow_{v}\right|$, then we construct two post-3/2 candidates for $\rho_{v}^{*}$, and then assign $\rho_{v}^{*}$ to the cheapest among the two, proving that its cost has to be lower than $\mathcal{C}\left(\rho \downarrow_{v}\right)$.

(a) All the changes of $v$ to $\mathrm{w}_{v}$ are done using action $a_{1}^{\mathrm{w}}$, and then the largest possible number of changes to $\mathrm{b}_{v}$ are done using action $a_{1}^{\mathrm{b}}$, with the remaining changes to $\mathrm{b}_{v}$ being done using action $\mathrm{a}_{2}^{\mathrm{b}}$. For this candidate for $\rho_{v}^{*}$, we set

$$
a_{i}^{*}= \begin{cases}a_{2}^{\mathrm{b}} & i=2 j, j \in \mathbb{N}, j \leq y-x \\ a_{1}^{\mathrm{b}} & i=2 j, j \in \mathbb{N}, y-x<j \leq y \\ a_{1}^{\mathrm{w}} & \text { otherwise }\end{cases}
$$

And the cost in this case is

$$
y \cdot \mathcal{C}\left(a_{1}^{\mathrm{w}}\right)+x \cdot \mathcal{C}\left(a_{1}^{\mathrm{b}}\right)+(y-x) \cdot \mathcal{C}\left(a_{2}^{\mathrm{b}}\right)
$$

The ordering constraints $>_{v}$ are set according to Eq. 75. Likewise, if $\rho_{w}^{*}=$ $\left\langle a_{1}, \ldots, a_{2 x}\right\rangle$, we set $>_{v, w}$ according to Eq. 96 above. For each $u \in \operatorname{pred}(v) \backslash$ $\{w\}$ we set $>_{v, u}$ according to Eq. 82 above. The relations $>_{v},>_{v, w}$ and $>_{v, u}$ are effectively identical to the previous case. Thus, we have $>_{v} \cup>_{v, u}$ and $>_{v} \cup>_{v, w}$ forming strict partial orders over the unions of the elements of $\rho_{v}^{*}$ and $\rho_{u}^{*}$, and $\rho_{v}^{*}$ and $\rho_{w}^{*}$, respectively.

From Eqs. 75, 82, 96 we can now derive that any linearization of $>_{v} \cup \bigcup_{w \in \operatorname{pred}(v)}>_{v, w}$ defines a sequence of actions that is applicable with respect to $\{v\} \cup \operatorname{pred}(v)$. In addition, $\left|\rho_{v}^{*}\right|=\left|\rho \downarrow_{v}\right|$ together with Eq. 118 implies that this action sequence provides to $v$ the value $G[v]$ if the latter is specified. 
(b) All the changes of $v$ to $\mathrm{b}_{v}$ are done using action $a_{1}^{\mathrm{b}}$, and then the largest possible number of changes to $\mathrm{w}_{v}$ are done using action $a_{1}^{\mathrm{w}}$, with the remaining changes to $\mathrm{w}_{v}$ being done using action $a_{2}^{\mathrm{w}}$. For this candidate for $\rho_{v}^{*}$, we set

$$
a_{i}^{*}= \begin{cases}a_{1}^{\mathrm{w}} & i=2 j-1, j \in \mathbb{N}, j \leq x \\ a_{2}^{\mathrm{w}} & i=2 j-1, j \in \mathbb{N}, x<j \leq y \\ a_{1}^{\mathrm{b}} & \text { otherwise }\end{cases}
$$

And the cost in this case is

$$
x \cdot \mathcal{C}\left(a_{1}^{\mathrm{w}}\right)+(y-x) \cdot \mathcal{C}\left(a_{2}^{\mathrm{w}}\right)+y \cdot \mathcal{C}\left(a_{1}^{\mathrm{b}}\right)
$$

The ordering constraints $>_{v}$ are set according to Eq. 75. Likewise, if $\rho_{w}^{*}=$ $\left\langle a_{1}, \ldots, a_{2 x}\right\rangle$, we set $\rangle_{v, w}$ according to Eq. 122 .

$$
>_{v, w}=\bigcup_{a_{i}^{*} \in \rho_{v}^{*}, a_{j} \in \rho_{w}^{*}}\left\{a_{i}^{*}<a_{j} \mid i \leq j<2 x\right\} \cup\left\{a_{j}<a_{i}^{*} \mid j<i, j<2 x\right\} \cup\left\{a_{i}^{*}<a_{2 x}\right\}
$$

For each $u \in \operatorname{pred}(v) \backslash\{w\}$ we set $>_{v, u}$ according to Eq. 86 above.

It is easy to verify that the relation $>_{v, w}$ defined by Eq. 122 is a strict total order over its domain. The relations $>_{v}$ and $>_{v, u}$ are effectively identical to the previous case. Thus, we have $>_{v} \cup>_{v, u}$ and $>_{v} \cup>_{v, w}$ forming strict partial orders over the unions of the elements of $\rho_{v}^{*}$ and $\rho_{u}^{*}$, and $\rho_{v}^{*}$ and $\rho_{w}^{*}$, respectively.

From Eqs. 75, 86, 122 we can now derive that any linearization of $>_{v} \cup \bigcup_{w \in \operatorname{pred}(v)}>_{v, w}$ defines a sequence of actions that is applicable with respect to $\{v\} \cup \operatorname{pred}(v)$. In addition, $\left|\rho_{v}^{*}\right|=\left|\rho \downarrow_{v}\right|$ together with Eq. 120 implies that this action sequence provides to $v$ the value $G[v]$ if the latter is specified.

Now, for each cost-optimal plan $\rho, \rho \downarrow_{v}$ cannot contain more than $y+x$ actions of both types $a_{1}^{\mathrm{w}}$ and $a_{1}^{\mathrm{b}}$ totally. Suppose to the contrary that $\rho \downarrow_{v}$ contain at least $y+x+1$ actions of types $a_{1}^{\mathrm{w}}$ and $a_{1}^{\mathrm{b}}$. Then it contains no more than $y-x-1$ actions of other types. Let $\mathrm{b}_{w} \cdot \mathbf{w}_{w} \cdot \ldots \cdot \mathbf{w}_{w}$ sequence of $2 y$ values of $w$ that support a cost-optimal plan for $v$ given that $w$ can change its value any number of times. Then each action of any other type will decrease the needed length of this sequence by at most 2 . Therefore at most $y-x-1$ actions of other type will decrease the length by at most $2 y-2 x-2$, and we are left with the sequence of length $\geq 2 y-(2 y-2 x-2)=2 x+2$. Therefore $\sigma_{w}$ cannot support more than $y+x$ actions of types $a_{1}^{\mathrm{w}}$ and $a_{1}^{\mathrm{b}}$. Now, suppose that in some given cost-optimal plan $\rho \downarrow_{v}$ for $v$ there are $\alpha$ actions of type $a_{1}^{\mathrm{w}}$ and $\beta$ actions of type $a_{1}^{\mathrm{b}}$. Then

$$
\alpha+\beta \leq y+x
$$

and

$$
\mathcal{C}\left(\rho \downarrow_{v}\right) \geq \alpha \cdot \mathcal{C}\left(a_{1}^{\mathrm{w}}\right)+(y-\alpha) \cdot \mathcal{C}\left(a_{2}^{\mathrm{w}}\right)+\beta \cdot \mathcal{C}\left(a_{1}^{\mathrm{b}}\right)+(y-\beta) \cdot \mathcal{C}\left(a_{2}^{\mathrm{b}}\right)
$$


For $(119) \leq(121)$, we have

$$
\mathcal{C}\left(a_{2}^{\mathrm{b}}\right)-\mathcal{C}\left(a_{1}^{\mathrm{b}}\right) \leq \mathcal{C}\left(a_{2}^{\mathrm{w}}\right)-\mathcal{C}\left(a_{1}^{\mathrm{w}}\right)
$$

Now suppose to the contrary that the plan in first case is not cost-optimal. Then from Eq. 124 we have

$$
\begin{aligned}
& \alpha \cdot \mathcal{C}\left(a_{1}^{\mathrm{w}}\right)+(y-\alpha) \cdot \mathcal{C}\left(a_{2}^{\mathrm{w}}\right)+\beta \cdot \mathcal{C}\left(a_{1}^{\mathrm{b}}\right)+(y-\beta) \cdot \mathcal{C}\left(a_{2}^{\mathrm{b}}\right)< \\
& y \cdot \mathcal{C}\left(a_{1}^{\mathrm{w}}\right)+x \cdot \mathcal{C}\left(a_{1}^{\mathrm{b}}\right)+(y-x) \cdot \mathcal{C}\left(a_{2}^{\mathrm{b}}\right)
\end{aligned}
$$

and from it

$$
(y-\alpha) \cdot\left(\mathcal{C}\left(a_{2}^{\mathrm{w}}\right)-\mathcal{C}\left(a_{1}^{\mathrm{w}}\right)\right)<(\beta-x) \cdot\left(\mathcal{C}\left(a_{2}^{\mathrm{b}}\right)-\mathcal{C}\left(a_{1}^{\mathrm{b}}\right)\right)
$$

From Eq. 123 we have $y-\alpha \geq \beta-x$, together with Eq. 125 contradicting Eq. 126 . For $(121) \leq(119)$, we have

$$
\mathcal{C}\left(a_{2}^{\mathrm{w}}\right)-\mathcal{C}\left(a_{1}^{\mathrm{w}}\right) \leq \mathcal{C}\left(a_{2}^{\mathrm{b}}\right)-\mathcal{C}\left(a_{1}^{\mathrm{b}}\right)
$$

Now suppose to the contrary that the plan in second case is not cost-optimal. Then from Eq. 124 we have

$$
\begin{aligned}
& \alpha \cdot \mathcal{C}\left(a_{1}^{\mathrm{w}}\right)+(y-\alpha) \cdot \mathcal{C}\left(a_{2}^{\mathrm{w}}\right)+\beta \cdot \mathcal{C}\left(a_{1}^{\mathrm{b}}\right)+(y-\beta) \cdot \mathcal{C}\left(a_{2}^{\mathrm{b}}\right)< \\
& x \cdot \mathcal{C}\left(a_{1}^{\mathrm{w}}\right)+(y-x) \cdot \mathcal{C}\left(a_{2}^{\mathrm{w}}\right)+y \cdot \mathcal{C}\left(a_{1}^{\mathrm{b}}\right)
\end{aligned}
$$

and from it

$$
(y-\beta) \cdot\left(\mathcal{C}\left(a_{2}^{\mathrm{b}}\right)-\mathcal{C}\left(a_{1}^{\mathrm{b}}\right)\right)<(\alpha-x) \cdot\left(\mathcal{C}\left(a_{2}^{\mathrm{w}}\right)-\mathcal{C}\left(a_{1}^{\mathrm{w}}\right)\right)
$$

From Eq. 123 we have $y-\beta \geq \alpha-x$, together with Eq. 127 contradicting Eq. 128.

(8) If $\left|\rho \downarrow_{v}\right|=2 y,\left|\sigma_{w}\right|=2 x+1,\left|\sigma_{w}\right|>\left|\rho \downarrow_{v}\right|$, then the actions of $\rho_{v}^{*}$ are set to

$$
a_{i}^{*}= \begin{cases}a_{\mathrm{w}_{v} \mid \mathrm{b}_{w}} & i=2 j-1, j \in \mathbb{N} \\ a_{\mathrm{b}_{v} \mid \mathrm{w}_{w}} & \text { otherwise }\end{cases}
$$

The ordering constraints $>_{v}$ are set according to Eq. 75. Likewise, if $\rho_{w}^{*}=$ $\left\langle a_{1}, \ldots, a_{2 x}\right\rangle$, we set $>_{v, w}$ according to Eq. 85 above. Finally, the ordering constraints $>_{v, w^{\prime}}$ for the rest of the parents $w^{\prime} \in \operatorname{pred}(v) \backslash\{u, w\}$ are set to empty sets. The relations $>_{v}$ and $>_{v, w}$ are identical to the previous case. Thus, we have $>_{v} \cup>_{v, w}$ forming strict partial order over the union of the elements of $\rho_{v}^{*}$ and $\rho_{w}^{*}$.

From Eqs. 75, 85 we can now derive that any linearization of $>_{v} \cup \bigcup_{w \in \operatorname{pred}(v)}>_{v, w}$ defines a sequence of actions that is applicable with respect to $\{v\} \cup \operatorname{pred}(v)$. In addition, $\left|\rho_{v}^{*}\right|=\left|\rho \downarrow_{v}\right|$ together with Eq. 129 implies that this action sequence provides to $v$ the value $G[v]$ if the latter is specified.

(IV) If $\gamma=\mathrm{w}_{w}, \delta=\mathrm{b}_{w}$, we distinguish between a few cases based on $\sigma_{w}$ and $\sigma_{v}$. 
(1) If $\left|\rho \downarrow_{v}\right|=2 y+1,\left|\sigma_{w}\right|=2 x,\left|\sigma_{w}\right| \leq\left|\rho \downarrow_{v}\right|$, then we construct two post-3/2 candidates for $\rho_{v}^{*}$, and then assign $\rho_{v}^{*}$ to the cheapest among the two, proving that its cost has to be lower than $\mathcal{C}\left(\rho \downarrow_{v}\right)$.

(a) All the changes of $v$ to $\mathrm{w}_{v}$ are done using action $a_{1}^{\mathrm{w}}$, and then the largest possible number of changes to $\mathrm{b}_{v}$ are done using action $a_{1}^{\mathrm{b}}$, with the remaining changes to $\mathrm{b}_{v}$ being done using action $a_{2}^{\mathrm{b}}$. For this candidate for $\rho_{v}^{*}$, we set

$$
a_{i}^{*}= \begin{cases}a_{2}^{\mathrm{b}} & i=2 j, j \in \mathbb{N}, j \leq y-x+1 \\ a_{1}^{\mathrm{b}} & i=2 j, j \in \mathbb{N}, y-x+1<j \leq y \\ a_{1}^{\mathrm{w}} & \text { otherwise }\end{cases}
$$

And the cost in this case is

$$
(y+1) \cdot \mathcal{C}\left(a_{1}^{\mathrm{w}}\right)+(x-1) \cdot \mathcal{C}\left(a_{1}^{\mathrm{b}}\right)+(y-x+1) \cdot \mathcal{C}\left(a_{2}^{\mathrm{b}}\right)
$$

The ordering constraints $>_{v}$ are set according to Eq. 75. Likewise, if $\rho_{w}^{*}=$ $\left\langle a_{1}, \ldots, a_{2 x-1}\right\rangle$, we set $>_{v, w}$ according to Eq. 132 .

$$
>_{v, w}=\bigcup_{a_{i}^{*} \in \rho_{v}^{*}, a_{j} \in \rho_{w}^{*}}\left\{a_{i}^{*}<a_{j} \mid i<j\right\} \cup\left\{a_{j}<a_{i}^{*} \mid j \leq i\right\}
$$

For each $u \in \operatorname{pred}(v) \backslash\{w\}$ we set $>_{v, u}$ according to Eq. 82 .

It is easy to verify that the relation $>_{v, w}$ defined by Eq. 132 is a strict total order over its domain. The relations $>_{v}$ and $>_{v, u}$ are effectively identical to the previous case. Thus, we have $>_{v} \cup>_{v, u}$ and $>_{v} \cup>_{v, w}$ forming strict partial orders over the unions of the elements of $\rho_{v}^{*}$ and $\rho_{u}^{*}$, and $\rho_{v}^{*}$ and $\rho_{w}^{*}$, respectively.

From Eqs. 75, 82, 132 we can now derive that any linearization of $>_{v} \cup \bigcup_{w \in \operatorname{pred}(v)}>_{v, w}$ defines a sequence of actions that is applicable with respect to $\{v\} \cup \operatorname{pred}(v)$. In addition, $\left|\rho_{v}^{*}\right|=\left|\rho \downarrow_{v}\right|$ together with Eq. 130 implies that this action sequence provides to $v$ the value $G[v]$ if the latter is specified.

(b) All the changes of $v$ to $\mathrm{b}_{v}$ are done using action $a_{1}^{\mathrm{b}}$, and then the largest possible number of changes to $\mathrm{w}_{v}$ are done using action $a_{1}^{\mathrm{w}}$, with the remaining changes to $\mathrm{w}_{v}$ being done using action $a_{2}^{\mathrm{w}}$. For this candidate for $\rho_{v}^{*}$, we set

$$
a_{i}^{*}= \begin{cases}a_{2}^{\mathrm{w}} & i=2 j-1, j \in \mathbb{N}, j \leq y-x+1 \\ a_{1}^{\mathrm{w}} & i=2 j-1, j \in \mathbb{N}, y-x+1<j \leq y+1 \\ a_{1}^{\mathrm{b}} & \text { otherwise }\end{cases}
$$

And the cost in this case is

$$
x \cdot \mathcal{C}\left(a_{1}^{\mathrm{w}}\right)+(y+1-x) \cdot \mathcal{C}\left(a_{2}^{\mathrm{w}}\right)+y \cdot \mathcal{C}\left(a_{1}^{\mathrm{b}}\right)
$$

The ordering constraints $>_{v}$ are set according to Eq. 75. Likewise, if $\rho_{w}^{*}=$ $\left\langle a_{1}, \ldots, a_{2 x-1}\right\rangle$, we set $\rangle_{v, w}$ according to Eq. 135 .

$>_{v, w}=\bigcup_{a_{i}^{*} \in \rho_{v}^{*}, a_{j} \in \rho_{w}^{*}}\left\{a_{i}^{*}<a_{j} \mid i \leq 2 y-2 x+1+j\right\} \cup\left\{a_{j}<a_{i}^{*} \mid i>2 y-2 x+1+j\right\}$ 
For each $u \in \operatorname{pred}(v) \backslash\{w\}$ we set $>_{v, u}$ according to Eq. 86 .

It is easy to verify that the relation $>_{v, w}$ defined by Eq. 135 is a strict total order over its domain. The relations $>_{v}$ and $>_{v, u}$ are effectively identical to the previous case. Thus, we have $>_{v} \cup>_{v, u}$ and $>_{v} \cup>_{v, w}$ forming strict partial orders over the unions of the elements of $\rho_{v}^{*}$ and $\rho_{u}^{*}$, and $\rho_{v}^{*}$ and $\rho_{w}^{*}$, respectively.

From Eqs. 75, 86, 135 we can now derive that any linearization of $>_{v} \cup \bigcup_{w \in \operatorname{pred}(v)}>_{v, w}$ defines a sequence of actions that is applicable with respect to $\{v\} \cup \operatorname{pred}(v)$. In addition, $\left|\rho_{v}^{*}\right|=\left|\rho \downarrow_{v}\right|$ together with Eq. 133 implies that this action sequence provides to $v$ the value $G[v]$ if the latter is specified.

Now, for each cost-optimal plan $\rho, \rho \downarrow_{v}$ cannot contain more than $y+x$ actions of both types $a_{1}^{w}$ and $a_{1}^{\mathrm{b}}$ totally. Suppose to the contrary that $\rho \downarrow_{v}$ contain at least $y+x+1$ actions of types $a_{1}^{\mathrm{w}}$ and $a_{1}^{\mathrm{b}}$. Then it contains no more than $y-x$ actions of other types. Let $\mathrm{w}_{w} \cdot \mathrm{w}_{b} \cdot \ldots \cdot \mathrm{w}_{w}$ sequence of $2 y+1$ values of $w$ that support a cost-optimal plan for $v$ given that $w$ can change its value any number of times. Then each action of any other type will decrease the needed length of this sequence by at most 2 . Therefore at most $y-x$ actions of other type will decrease the length by at most $2 y-2 x$, and we are left with the sequence of length $\geq 2 y+1-(2 y-2 x)=2 x+1$. Therefore $\sigma_{w}$ cannot support more than $y+x$ actions of types $a_{1}^{\mathrm{w}}$ and $a_{1}^{\mathrm{b}}$. Now, suppose that in some given cost-optimal plan $\rho \downarrow_{v}$ for $v$ there are $\alpha$ actions of type $a_{1}^{\mathrm{w}}$ and $\beta$ actions of type $a_{1}^{\mathrm{b}}$. Then

$$
\alpha+\beta \leq y+x
$$

and

$$
\mathcal{C}\left(\rho \downarrow_{v}\right) \geq \alpha \cdot \mathcal{C}\left(a_{1}^{\mathrm{w}}\right)+(y+1-\alpha) \cdot \mathcal{C}\left(a_{2}^{\mathrm{w}}\right)+\beta \cdot \mathcal{C}\left(a_{1}^{\mathrm{b}}\right)+(y-\beta) \cdot \mathcal{C}\left(a_{2}^{\mathrm{b}}\right)
$$

For $(131) \leq(134)$, we have

$$
\mathcal{C}\left(a_{2}^{\mathrm{b}}\right)-\mathcal{C}\left(a_{1}^{\mathrm{b}}\right) \leq \mathcal{C}\left(a_{2}^{\mathrm{w}}\right)-\mathcal{C}\left(a_{1}^{\mathrm{w}}\right)
$$

Now suppose to the contrary that the plan in first case is not cost-optimal. Then from Eq. 137 we have

$$
\begin{aligned}
& \alpha \cdot \mathcal{C}\left(a_{1}^{\mathrm{w}}\right)+(y+1-\alpha) \cdot \mathcal{C}\left(a_{2}^{\mathrm{w}}\right)+\beta \cdot \mathcal{C}\left(a_{1}^{\mathrm{b}}\right)+(y-\beta) \cdot \mathcal{C}\left(a_{2}^{\mathrm{b}}\right)< \\
& (y+1) \cdot \mathcal{C}\left(a_{1}^{\mathrm{w}}\right)+(x-1) \cdot \mathcal{C}\left(a_{1}^{\mathrm{b}}\right)+(y-x+1) \cdot \mathcal{C}\left(a_{2}^{\mathrm{b}}\right)
\end{aligned}
$$

and from it

$$
(y+1-\alpha) \cdot\left(\mathcal{C}\left(a_{2}^{\mathrm{w}}\right)-\mathcal{C}\left(a_{1}^{\mathrm{w}}\right)\right)<(\beta-x+1) \cdot\left(\mathcal{C}\left(a_{2}^{\mathrm{b}}\right)-\mathcal{C}\left(a_{1}^{\mathrm{b}}\right)\right)
$$

From Eq. 136 we have $y+1-\alpha \geq \beta-x+1$, together with Eq. 138 contradicting Eq. 139.

For $(134) \leq(131)$, we have

$$
\mathcal{C}\left(a_{2}^{\mathrm{w}}\right)-\mathcal{C}\left(a_{1}^{\mathrm{w}}\right) \leq \mathcal{C}\left(a_{2}^{\mathrm{b}}\right)-\mathcal{C}\left(a_{1}^{\mathrm{b}}\right)
$$


Now suppose to the contrary that the plan in second case is not cost-optimal. Then from Eq. 137 we have

$$
\begin{aligned}
& \alpha \cdot \mathcal{C}\left(a_{1}^{\mathrm{w}}\right)+(y+1-\alpha) \cdot \mathcal{C}\left(a_{2}^{\mathrm{w}}\right)+\beta \cdot \mathcal{C}\left(a_{1}^{\mathrm{b}}\right)+(y-\beta) \cdot \mathcal{C}\left(a_{2}^{\mathrm{b}}\right)< \\
& x \cdot \mathcal{C}\left(a_{1}^{\mathrm{w}}\right)+(y-x+1) \cdot \mathcal{C}\left(a_{2}^{\mathrm{w}}\right)+y \cdot \mathcal{C}\left(a_{1}^{\mathrm{b}}\right)
\end{aligned}
$$

and from it

$$
(y-\beta) \cdot\left(\mathcal{C}\left(a_{2}^{\mathrm{b}}\right)-\mathcal{C}\left(a_{1}^{\mathrm{b}}\right)\right)<(\alpha-x) \cdot\left(\mathcal{C}\left(a_{2}^{\mathrm{w}}\right)-\mathcal{C}\left(a_{1}^{\mathrm{w}}\right)\right)
$$

From Eq. 136 we have $y-\beta \geq \alpha-x$, together with Eq. 140 contradicting Eq. 141.

(2) If $\left|\rho \downarrow_{v}\right|=2 y+1,\left|\sigma_{w}\right|=2 x,\left|\sigma_{w}\right|>\left|\rho \downarrow_{v}\right|$, then the actions of $\rho_{v}^{*}$ are set to

$$
a_{i}^{*}= \begin{cases}a_{\mathrm{w}_{v} \mid \mathrm{w}_{w}} & i=2 j-1, j \in \mathbb{N} \\ a_{\mathrm{b}_{v} \mid \mathrm{b}_{w}} & \text { otherwise }\end{cases}
$$

The ordering constraints $>_{v}$ are set according to Eq. 75. Likewise, if $\rho_{w}^{*}=$ $\left\langle a_{1}, \ldots, a_{2 x-1}\right\rangle$, we set $>_{v, w}$ according to Eq. 132 above. Finally, the ordering constraints $>_{v, w^{\prime}}$ for the rest of the parents $w^{\prime} \in \operatorname{pred}(v) \backslash\{u, w\}$ are set to empty sets. The relations $>_{v}$ and $>_{v, w}$ are identical to the previous case. Thus, we have $>_{v} \cup>_{v, w}$ forming strict partial order over the union of the elements of $\rho_{v}^{*}$ and $\rho_{w}^{*}$.

From Eqs. 75, 132 we can now derive that any linearization of $>_{v} \cup \bigcup_{w \in \operatorname{pred}(v)}>_{v, w}$ defines a sequence of actions that is applicable with respect to $\{v\} \cup \operatorname{pred}(v)$. In addition, $\left|\rho_{v}^{*}\right|=\left|\rho \downarrow_{v}\right|$ together with Eq. 142 implies that this action sequence provides to $v$ the value $G[v]$ if the latter is specified.

(3) If $\left|\rho \downarrow_{v}\right|=2 y,\left|\sigma_{w}\right|=2 x,\left|\sigma_{w}\right| \leq\left|\rho \downarrow_{v}\right|+1$, then we construct two post-3/2 candidates for $\rho_{v}^{*}$, and then assign $\rho_{v}^{*}$ to the cheapest among the two, proving that its cost has to be lower than $\mathcal{C}\left(\rho \downarrow_{v}\right)$.

(a) All the changes of $v$ to $\mathrm{w}_{v}$ are done using action $a_{1}^{\mathrm{w}}$, and then the largest possible number of changes to $\mathrm{b}_{v}$ are done using action $a_{1}^{\mathrm{b}}$, with the remaining changes to $\mathrm{b}_{v}$ being done using action $\mathrm{a}_{2}^{\mathrm{b}}$. For this candidate for $\rho_{v}^{*}$, we set

$$
a_{i}^{*}= \begin{cases}a_{1}^{\mathrm{b}} & i=2 j, j \in \mathbb{N}, j<x \\ a_{2}^{\mathrm{b}} & i=2 j, j \in \mathbb{N}, x \leq j \leq y \\ a_{1}^{\mathrm{w}} & \text { otherwise }\end{cases}
$$

And the cost in this case is

$$
y \cdot \mathcal{C}\left(a_{1}^{\mathrm{w}}\right)+(x-1) \cdot \mathcal{C}\left(a_{1}^{\mathrm{b}}\right)+(y-x+1) \cdot \mathcal{C}\left(a_{2}^{\mathrm{b}}\right)
$$

The ordering constraints $>_{v}$ are set according to Eq. 75. Likewise, if $\rho_{w}^{*}=$ $\left\langle a_{1}, \ldots, a_{2 x-1}\right\rangle$, we set $\rangle_{v, w}$ according to Eq. 132 above.

For each $u \in \operatorname{pred}(v) \backslash\{w\}$ we set $>_{v, u}$ according to Eq. 82 .

The relations $>_{v},>_{v, w}$ and $>_{v, u}$ are effectively identical to the previous case. Thus, we have $>_{v} \cup>_{v, u}$ and $>_{v} \cup>_{v, w}$ forming strict partial orders over the unions of the elements of $\rho_{v}^{*}$ and $\rho_{u}^{*}$, and $\rho_{v}^{*}$ and $\rho_{w}^{*}$, respectively. 
From Eqs. 75, 82, 132 we can now derive that any linearization of $>_{v} \cup \bigcup_{w \in \operatorname{pred}(v)}>_{v, w}$ defines a sequence of actions that is applicable with respect to $\{v\} \cup \operatorname{pred}(v)$. In addition, $\left|\rho_{v}^{*}\right|=\left|\rho \downarrow_{v}\right|$ together with Eq. 143 implies that this action sequence provides to $v$ the value $G[v]$ if the latter is specified.

(b) All the changes of $v$ to $\mathrm{b}_{v}$ are done using action $a_{1}^{\mathrm{b}}$, and then the largest possible number of changes to $\mathrm{w}_{v}$ are done using action $a_{1}^{\mathrm{w}}$, with the remaining changes to $\mathrm{w}_{v}$ being done using action $a_{2}^{\mathrm{w}}$. For this candidate for $\rho_{v}^{*}$, we set

$$
a_{i}^{*}= \begin{cases}a_{2}^{\mathrm{w}} & i=2 j-1, j \in \mathbb{N}, j \leq y-x+1 \\ a_{1}^{\mathrm{w}} & i=2 j-1, j \in \mathbb{N}, y-x+1<j \leq y \\ a_{1}^{\mathrm{b}} & \text { otherwise }\end{cases}
$$

And the cost in this case is

$$
(x-1) \cdot \mathcal{C}\left(a_{1}^{\mathrm{w}}\right)+(y-x+1) \cdot \mathcal{C}\left(a_{2}^{\mathrm{w}}\right)+y \cdot \mathcal{C}\left(a_{1}^{\mathrm{b}}\right)
$$

The ordering constraints $>_{v}$ are set according to Eq. 75. Likewise, if $\rho_{w}^{*}=$ $\left\langle a_{1}, \ldots, a_{2 x-1}\right\rangle$, we set $>_{v, w}$ according to Eq. 135 above.

For each $u \in \operatorname{pred}(v) \backslash\{w\}$ we set $>_{v, u}$ according to Eq. 86 .

The relations $>_{v},>_{v, w}$ and $>_{v, u}$ are effectively identical to the previous case. Thus, we have $>_{v} \cup>_{v, u}$ and $>_{v} \cup>_{v, w}$ forming strict partial orders over the unions of the elements of $\rho_{v}^{*}$ and $\rho_{u}^{*}$, and $\rho_{v}^{*}$ and $\rho_{w}^{*}$, respectively.

From Eqs. 75, 86, 135 we can now derive that any linearization of $>_{v} \cup \bigcup_{w \in \operatorname{pred}(v)}>_{v, w}$ defines a sequence of actions that is applicable with respect to $\{v\} \cup \operatorname{pred}(v)$. In addition, $\left|\rho_{v}^{*}\right|=\left|\rho \downarrow_{v}\right|$ together with Eq. 145 implies that this action sequence provides to $v$ the value $G[v]$ if the latter is specified.

Now, for each cost-optimal plan $\rho, \rho \downarrow_{v}$ cannot contain more than $y+x-1$ actions of both types $a_{1}^{\mathrm{w}}$ and $a_{1}^{\mathrm{b}}$ totally. Suppose to the contrary that $\rho \downarrow_{v}$ contain at least $y+x$ actions of types $a_{1}^{\mathrm{w}}$ and $a_{1}^{\mathrm{b}}$. Then it contains no more than $y-x$ actions of other types. Let $\mathrm{w}_{w} \cdot \mathrm{b}_{w} \cdot \ldots \cdot \mathrm{b}_{w}$ sequence of $2 y$ values of $w$ that support a cost-optimal plan for $v$ given that $w$ can change its value any number of times. Then each action of any other type will decrease the needed length of this sequence by at most 2 . Therefore at most $y-x$ actions of other type will decrease the length by at most $2 y-2 x$, and we are left with the sequence of length $\geq 2 y-(2 y-2 x)=2 x$, which have to be a subsequence of $\sigma_{w}$, contradicting with the fact that $\sigma_{w}$ is of the same or smaller size and starts with a different character. Therefore $\sigma_{w}$ cannot support more than $y+x$ actions of types $a_{1}^{w}$ and $a_{1}^{\mathrm{b}}$. Now, suppose that in some given cost-optimal plan $\rho \downarrow_{v}$ for $v$ there are $\alpha$ actions of type $a_{1}^{\mathrm{w}}$ and $\beta$ actions of type $a_{1}^{\mathrm{b}}$. Then

$$
\alpha+\beta \leq y+x
$$

and

$$
\mathcal{C}\left(\rho \downarrow_{v}\right) \geq \alpha \cdot \mathcal{C}\left(a_{1}^{\mathrm{w}}\right)+(y-\alpha) \cdot \mathcal{C}\left(a_{2}^{\mathrm{w}}\right)+\beta \cdot \mathcal{C}\left(a_{1}^{\mathrm{b}}\right)+(y-\beta) \cdot \mathcal{C}\left(a_{2}^{\mathrm{b}}\right)
$$


For $(144) \leq(146)$, we have

$$
\mathcal{C}\left(a_{2}^{\mathrm{b}}\right)-\mathcal{C}\left(a_{1}^{\mathrm{b}}\right) \leq \mathcal{C}\left(a_{2}^{\mathrm{w}}\right)-\mathcal{C}\left(a_{1}^{\mathrm{w}}\right)
$$

Now suppose to the contrary that the plan in first case is not cost-optimal. Then from Eq. 148 we have

$$
\begin{aligned}
& \alpha \cdot \mathcal{C}\left(a_{1}^{\mathrm{w}}\right)+(y-\alpha) \cdot \mathcal{C}\left(a_{2}^{\mathrm{w}}\right)+\beta \cdot \mathcal{C}\left(a_{1}^{\mathrm{b}}\right)+(y-\beta) \cdot \mathcal{C}\left(a_{2}^{\mathrm{b}}\right)< \\
& y \cdot \mathcal{C}\left(a_{1}^{\mathrm{w}}\right)+x \cdot \mathcal{C}\left(a_{1}^{\mathrm{b}}\right)+(y-x) \cdot \mathcal{C}\left(a_{2}^{\mathrm{b}}\right)
\end{aligned}
$$

and from it

$$
(y-\alpha) \cdot\left(\mathcal{C}\left(a_{2}^{\mathrm{w}}\right)-\mathcal{C}\left(a_{1}^{\mathrm{w}}\right)\right)<(\beta-x) \cdot\left(\mathcal{C}\left(a_{2}^{\mathrm{b}}\right)-\mathcal{C}\left(a_{1}^{\mathrm{b}}\right)\right)
$$

From Eq. 147 we have $y-\alpha \geq \beta-x$, together with Eq. 149 contradicting Eq. 150. For $(146) \leq(144)$, we have

$$
\mathcal{C}\left(a_{2}^{\mathrm{w}}\right)-\mathcal{C}\left(a_{1}^{\mathrm{w}}\right) \leq \mathcal{C}\left(a_{2}^{\mathrm{b}}\right)-\mathcal{C}\left(a_{1}^{\mathrm{b}}\right)
$$

Now suppose to the contrary that the plan in second case is not cost-optimal. Then from Eq. 148 we have

$$
\begin{aligned}
& \alpha \cdot \mathcal{C}\left(a_{1}^{\mathrm{w}}\right)+(y-\alpha) \cdot \mathcal{C}\left(a_{2}^{\mathrm{w}}\right)+\beta \cdot \mathcal{C}\left(a_{1}^{\mathrm{b}}\right)+(y-\beta) \cdot \mathcal{C}\left(a_{2}^{\mathrm{b}}\right)< \\
& x \cdot \mathcal{C}\left(a_{1}^{\mathrm{w}}\right)+(y-x) \cdot \mathcal{C}\left(a_{2}^{\mathrm{w}}\right)+y \cdot \mathcal{C}\left(a_{1}^{\mathrm{b}}\right)
\end{aligned}
$$

and from it

$$
(y-\beta) \cdot\left(\mathcal{C}\left(a_{2}^{\mathrm{b}}\right)-\mathcal{C}\left(a_{1}^{\mathrm{b}}\right)\right)<(\alpha-x) \cdot\left(\mathcal{C}\left(a_{2}^{\mathrm{w}}\right)-\mathcal{C}\left(a_{1}^{\mathrm{w}}\right)\right)
$$

From Eq. 147 we have $y-\beta \geq \alpha-x$, together with Eq. 151 contradicting Eq. 152.

(4) If $\left|\rho \downarrow_{v}\right|=2 y,\left|\sigma_{w}\right|=2 x,\left|\sigma_{w}\right|>\left|\rho \downarrow_{v}\right|+1$, then the actions of $\rho_{v}^{*}$ are set to

$$
a_{i}^{*}= \begin{cases}a_{\mathrm{w}_{v} \mid \mathrm{w}_{w}} & i=2 j-1, j \in \mathbb{N} \\ a_{\mathrm{b}_{v} \mid \mathrm{b}_{w}} & \text { otherwise }\end{cases}
$$

The ordering constraints $>_{v}$ are set according to Eq. 75. Likewise, if $\rho_{w}^{*}=$ $\left\langle a_{1}, \ldots, a_{2 x-1}\right\rangle$, we set $>_{v, w}$ according to Eq. 132 above. Finally, the ordering constraints $>_{v, w^{\prime}}$ for the rest of the parents $w^{\prime} \in \operatorname{pred}(v) \backslash\{u, w\}$ are set to empty sets. The relations $>_{v}$ and $>_{v, w}$ are identical to the previous case. Thus, we have $>_{v} \cup>_{v, w}$ forming strict partial order over the union of the elements of $\rho_{v}^{*}$ and $\rho_{w}^{*}$.

From Eqs. 75, 132 we can now derive that any linearization of $>_{v} \cup \bigcup_{w \in \operatorname{pred}(v)}>_{v, w}$ defines a sequence of actions that is applicable with respect to $\{v\} \cup \operatorname{pred}(v)$. In addition, $\left|\rho_{v}^{*}\right|=\left|\rho \downarrow_{v}\right|$ together with Eq. 153 implies that this action sequence provides to $v$ the value $G[v]$ if the latter is specified.

(5) If $\left|\rho \downarrow_{v}\right|=2 y+1,\left|\sigma_{w}\right|=2 x+1,\left|\sigma_{w}\right| \leq\left|\rho \downarrow_{v}\right|+1$, then we construct two post-3/2 candidates for $\rho_{v}^{*}$, and then assign $\rho_{v}^{*}$ to the cheapest among the two, proving that its cost has to be lower than $\mathcal{C}\left(\rho \downarrow_{v}\right)$. 
(a) All the changes of $v$ to $\mathrm{w}_{v}$ are done using action $a_{1}^{\mathrm{w}}$, and then the largest possible number of changes to $\mathrm{b}_{v}$ are done using action $a_{1}^{b}$, with the remaining changes to $\mathrm{b}_{v}$ being done using action $\mathrm{a}_{2}^{\mathrm{b}}$. For this candidate for $\rho_{v}^{*}$, we set

$$
a_{i}^{*}= \begin{cases}a_{1}^{\mathrm{b}} & i=2 j, j \in \mathbb{N}, j<x \\ a_{2}^{\mathrm{b}} & i=2 j, j \in \mathbb{N}, x \leq j \leq y \\ a_{1}^{\mathrm{w}} & \text { otherwise }\end{cases}
$$

And the cost in this case is

$$
(y+1) \cdot \mathcal{C}\left(a_{1}^{\mathrm{w}}\right)+(x-1) \cdot \mathcal{C}\left(a_{1}^{\mathrm{b}}\right)+(y-x+1) \cdot \mathcal{C}\left(a_{2}^{\mathrm{b}}\right)
$$

The ordering constraints $>_{v}$ are set according to Eq. 75. Likewise, if $\rho_{w}^{*}=$ $\left\langle a_{1}, \ldots, a_{2 x}\right\rangle$, we set $\rangle_{v, w}$ according to Eq. 156 .

$$
>_{v, w}=\bigcup_{a_{i}^{*} \in \rho_{v}^{*}, a_{j} \in \rho_{w}^{*}}\left\{a_{i}^{*}<a_{j} \mid i<j<2 x\right\} \cup\left\{a_{j}<a_{i}^{*} \mid j \leq i, j<2 x\right\} \cup\left\{a_{i}^{*}<a_{2 x}\right\}
$$

For each $u \in \operatorname{pred}(v) \backslash\{w\}$ we set $>_{v, u}$ according to Eq. 82 .

It is easy to verify that the relation $>_{v, w}$ defined by Eq. 156 is a strict total order over its domain. The relations $>_{v}$ and $>_{v, u}$ are effectively identical to the previous case. Thus, we have $>_{v} \cup>_{v, u}$ and $>_{v} \cup>_{v, w}$ forming strict partial orders over the unions of the elements of $\rho_{v}^{*}$ and $\rho_{u}^{*}$, and $\rho_{v}^{*}$ and $\rho_{w}^{*}$, respectively.

From Eqs. 75, 82, 156 we can now derive that any linearization of $>_{v} \cup \bigcup_{w \in \operatorname{pred}(v)}>_{v, w}$ defines a sequence of actions that is applicable with respect to $\{v\} \cup \operatorname{pred}(v)$. In addition, $\left|\rho_{v}^{*}\right|=\left|\rho \downarrow_{v}\right|$ together with Eq. 154 implies that this action sequence provides to $v$ the value $G[v]$ if the latter is specified.

(b) All the changes of $v$ to $\mathrm{b}_{v}$ are done using action $a_{1}^{\mathrm{b}}$, and then the largest possible number of changes to $\mathrm{w}_{v}$ are done using action $a_{1}^{\mathrm{w}}$, with the remaining changes to $\mathrm{w}_{v}$ being done using action $a_{2}^{\mathrm{w}}$. For this candidate for $\rho_{v}^{*}$, we set

$$
a_{i}^{*}= \begin{cases}a_{2}^{\mathrm{w}} & i=2 j-1, j \in \mathbb{N}, j \leq y-x \text { or } j=y+1 \\ a_{1}^{\mathrm{w}} & i=2 j-1, j \in \mathbb{N}, y-x<j \leq y \\ a_{1}^{\mathrm{b}} & \text { otherwise }\end{cases}
$$

And the cost in this case is

$$
x \cdot \mathcal{C}\left(a_{1}^{\mathrm{w}}\right)+(y+1-x) \cdot \mathcal{C}\left(a_{2}^{\mathrm{w}}\right)+y \cdot \mathcal{C}\left(a_{1}^{\mathrm{b}}\right)
$$

The ordering constraints $>_{v}$ are set according to Eq. 75. Likewise, if $\rho_{w}^{*}=$ $\left\langle a_{1}, \ldots, a_{2 x-1}\right\rangle$, we set $\rangle_{v, w}$ according to Eq. 159 .

$$
>_{v, w}=\bigcup_{a_{i}^{*} \in \rho_{v}^{*}, a_{j} \in \rho_{w}^{*}}\left\{a_{i}^{*}<a_{j} \mid i<2 y-2 x+j\right\} \cup\left\{a_{j}<a_{i}^{*} \mid i \geq 2 y-2 x+j\right\}
$$


For each $u \in \operatorname{pred}(v) \backslash\{w\}$ we set $>_{v, u}$ according to Eq. 86 .

It is easy to verify that the relation $>_{v, w}$ defined by Eq. 159 is a strict total order over its domain. The relations $>_{v}$ and $>_{v, u}$ are effectively identical to the previous case. Thus, we have $>_{v} \cup>_{v, u}$ and $>_{v} \cup>_{v, w}$ forming strict partial orders over the unions of the elements of $\rho_{v}^{*}$ and $\rho_{u}^{*}$, and $\rho_{v}^{*}$ and $\rho_{w}^{*}$, respectively.

From Eqs. 75, 86, 159 we can now derive that any linearization of $>_{v} \cup \bigcup_{w \in \operatorname{pred}(v)}>_{v, w}$ defines a sequence of actions that is applicable with respect to $\{v\} \cup \operatorname{pred}(v)$. In addition, $\left|\rho_{v}^{*}\right|=\left|\rho \downarrow_{v}\right|$ together with Eq. 157 implies that this action sequence provides to $v$ the value $G[v]$ if the latter is specified.

Now, for each cost-optimal plan $\rho, \rho \downarrow_{v}$ cannot contain more than $y+x+1$ actions of both types $a_{1}^{\mathrm{w}}$ and $a_{1}^{\mathrm{b}}$ totally. Suppose to the contrary that $\rho \downarrow_{v}$ contain at least $y+x+2$ actions of types $a_{1}^{\mathrm{w}}$ and $a_{1}^{\mathrm{b}}$. Then it contains no more than $y-x-1$ actions of other types. Let $\mathrm{w}_{w} \cdot \mathrm{b}_{w} \cdot \ldots \cdot \mathrm{w}_{w}$ sequence of $2 y+1$ values of $w$ that support a cost-optimal plan for $v$ given that $w$ can change its value any number of times. Then each action of any other type will decrease the needed length of this sequence by at most 2 . Therefore at most $y-x-1$ actions of other type will decrease the length by at most $2 y-2 x-2$, and we are left with the sequence of length $\geq 2 y+1-(2 y-2 x-2)=2 x+3$. Therefore $\sigma_{w}$ cannot support more than $y+x+1$ actions of types $a_{1}^{\mathrm{w}}$ and $a_{1}^{\mathrm{b}}$. Now, suppose that in some given cost-optimal plan $\rho \downarrow_{v}$ for $v$ there are $\alpha$ actions of type $a_{1}^{\mathrm{W}}$ and $\beta$ actions of type $a_{1}^{\mathrm{b}}$. Then

$$
\alpha+\beta \leq y+x+1
$$

and

$$
\mathcal{C}\left(\rho \downarrow_{v}\right) \geq \alpha \cdot \mathcal{C}\left(a_{1}^{\mathrm{w}}\right)+(y+1-\alpha) \cdot \mathcal{C}\left(a_{2}^{\mathrm{w}}\right)+\beta \cdot \mathcal{C}\left(a_{1}^{\mathrm{b}}\right)+(y-\beta) \cdot \mathcal{C}\left(a_{2}^{\mathrm{b}}\right)
$$

For $(155) \leq(158)$, we have

$$
\mathcal{C}\left(a_{2}^{\mathrm{b}}\right)-\mathcal{C}\left(a_{1}^{\mathrm{b}}\right) \leq \mathcal{C}\left(a_{2}^{\mathrm{w}}\right)-\mathcal{C}\left(a_{1}^{\mathrm{w}}\right)
$$

Now suppose to the contrary that the plan in first case is not cost-optimal. Then from Eq. 161 we have

$$
\begin{aligned}
& \alpha \cdot \mathcal{C}\left(a_{1}^{\mathrm{w}}\right)+(y+1-\alpha) \cdot \mathcal{C}\left(a_{2}^{\mathrm{w}}\right)+\beta \cdot \mathcal{C}\left(a_{1}^{\mathrm{b}}\right)+(y-\beta) \cdot \mathcal{C}\left(a_{2}^{\mathrm{b}}\right)< \\
& (y+1) \cdot \mathcal{C}\left(a_{1}^{\mathrm{w}}\right)+x \cdot \mathcal{C}\left(a_{1}^{\mathrm{b}}\right)+(y-x) \cdot \mathcal{C}\left(a_{2}^{\mathrm{b}}\right)
\end{aligned}
$$

and from it

$$
(y+1-\alpha) \cdot\left(\mathcal{C}\left(a_{2}^{\mathrm{w}}\right)-\mathcal{C}\left(a_{1}^{\mathrm{w}}\right)\right)<(\beta-x) \cdot\left(\mathcal{C}\left(a_{2}^{\mathrm{b}}\right)-\mathcal{C}\left(a_{1}^{\mathrm{b}}\right)\right)
$$

From Eq. 160 we have $y+1-\alpha \geq \beta-x$, together with Eq. 162 contradicting Eq. 163.

For $(158) \leq(155)$, we have

$$
\mathcal{C}\left(a_{2}^{\mathrm{w}}\right)-\mathcal{C}\left(a_{1}^{\mathrm{w}}\right) \leq \mathcal{C}\left(a_{2}^{\mathrm{b}}\right)-\mathcal{C}\left(a_{1}^{\mathrm{b}}\right)
$$


Now suppose to the contrary that the plan in second case is not cost-optimal. Then from Eq. 161 we have

$$
\begin{aligned}
& \alpha \cdot \mathcal{C}\left(a_{1}^{\mathrm{w}}\right)+(y+1-\alpha) \cdot \mathcal{C}\left(a_{2}^{\mathrm{w}}\right)+\beta \cdot \mathcal{C}\left(a_{1}^{\mathrm{b}}\right)+(y-\beta) \cdot \mathcal{C}\left(a_{2}^{\mathrm{b}}\right)< \\
& \left(x+1 \cdot \mathcal{C}\left(a_{1}^{\mathrm{w}}\right)+(y-x) \cdot \mathcal{C}\left(a_{2}^{\mathrm{w}}\right)+y \cdot \mathcal{C}\left(a_{1}^{\mathrm{b}}\right)\right.
\end{aligned}
$$

and from it

$$
(y-\beta) \cdot\left(\mathcal{C}\left(a_{2}^{\mathrm{b}}\right)-\mathcal{C}\left(a_{1}^{\mathrm{b}}\right)\right)<(\alpha-x-1) \cdot\left(\mathcal{C}\left(a_{2}^{\mathrm{w}}\right)-\mathcal{C}\left(a_{1}^{\mathrm{w}}\right)\right)
$$

From Eq. 160 we have $y-\beta \geq \alpha-x-1$, together with Eq. 164 contradicting Eq. 165.

(6) If $\left|\rho \downarrow_{v}\right|=2 y+1,\left|\sigma_{w}\right|=2 x+1,\left|\sigma_{w}\right|>\left|\rho \downarrow_{v}\right|+1$, then the actions of $\rho_{v}^{*}$ are set to

$$
a_{i}^{*}= \begin{cases}a_{\mathrm{w}_{v} \mid \mathrm{w}_{w}} & i=2 j-1, j \in \mathbb{N} \\ a_{\mathrm{b}_{v} \mid \mathrm{b}_{w}} & \text { otherwise }\end{cases}
$$

The ordering constraints $>_{v}$ are set according to Eq. 75. Likewise, if $\rho_{w}^{*}=$ $\left\langle a_{1}, \ldots, a_{2 x-1}\right\rangle$, we set $>_{v, w}$ according to Eq. 132 above.

Finally, the ordering constraints $>_{v, w^{\prime}}$ for the rest of the parents $w^{\prime} \in \operatorname{pred}(v) \backslash$ $\{u, w\}$ are set to empty sets.

The relations $>_{v}$ and $>_{v, w}$ are identical to the previous case. Thus, we have $>_{v} \cup>_{v, w}$ forming strict partial order over the union of the elements of $\rho_{v}^{*}$ and $\rho_{w}^{*}$.

From Eqs. 75, 132 we can now derive that any linearization of $>_{v} \cup \bigcup_{w \in \operatorname{pred}(v)}>_{v, w}$ defines a sequence of actions that is applicable with respect to $\{v\} \cup \operatorname{pred}(v)$. In addition, $\left|\rho_{v}^{*}\right|=\left|\rho \downarrow_{v}\right|$ together with Eq. 166 implies that this action sequence provides to $v$ the value $G[v]$ if the latter is specified.

(7) If $\left|\rho \downarrow_{v}\right|=2 y,\left|\sigma_{w}\right|=2 x+1,\left|\sigma_{w}\right| \leq\left|\rho \downarrow_{v}\right|$, then we construct two post-3/2 candidates for $\rho_{v}^{*}$, and then assign $\rho_{v}^{*}$ to the cheapest among the two, proving that its cost has to be lower than $\mathcal{C}\left(\rho \downarrow_{v}\right)$.

(a) All the changes of $v$ to $\mathrm{w}_{v}$ are done using action $a_{1}^{\mathrm{w}}$, and then the largest possible number of changes to $\mathrm{b}_{v}$ are done using action $a_{1}^{\mathrm{b}}$, with the remaining changes to $\mathrm{b}_{v}$ being done using action $\mathrm{a}_{2}^{\mathrm{b}}$. For this candidate for $\rho_{v}^{*}$, we set

$$
a_{i}^{*}= \begin{cases}a_{2}^{\mathrm{b}} & i=2 j, j \in \mathbb{N}, j \leq y-x \\ a_{1}^{\mathrm{b}} & i=2 j, j \in \mathbb{N}, y-x<j \leq y \\ a_{1}^{\mathrm{w}} & \text { otherwise }\end{cases}
$$

And the cost in this case is

$$
y \cdot \mathcal{C}\left(a_{1}^{\mathrm{w}}\right)+x \cdot \mathcal{C}\left(a_{1}^{\mathrm{b}}\right)+(y-x) \cdot \mathcal{C}\left(a_{2}^{\mathrm{b}}\right)
$$

The ordering constraints $>_{v}$ are set according to Eq. 75. Likewise, if $\rho_{w}^{*}=$ $\left\langle a_{1}, \ldots, a_{2 x}\right\rangle$, we set $\rangle_{v, w}$ according to Eq. 169 .

$>_{v, w}=\bigcup_{a_{i}^{*} \in \rho_{v}^{*}, a_{j} \in \rho_{w}^{*}}\left\{a_{i}^{*}<a_{j} \mid i<2 y-2 x+j, j>1\right\} \cup\left\{a_{j}<a_{i}^{*} \mid i \geq 2 y-2 x+j\right\} \cup\left\{a_{1}<a_{i}^{*}\right\}$ 
For each $u \in \operatorname{pred}(v) \backslash\{w\}$ we set $>_{v, u}$ according to Eq. 82 .

It is easy to verify that the relation $>_{v, w}$ defined by Eq. 169 is a strict total order over its domain. The relations $>_{v}$ and $>_{v, u}$ are effectively identical to the previous case. Thus, we have $>_{v} \cup>_{v, u}$ and $>_{v} \cup>_{v, w}$ forming strict partial orders over the unions of the elements of $\rho_{v}^{*}$ and $\rho_{u}^{*}$, and $\rho_{v}^{*}$ and $\rho_{w}^{*}$, respectively.

From Eqs. 75, 82, 169 we can now derive that any linearization of $>_{v} \cup \bigcup_{w \in \operatorname{pred}(v)}>_{v, w}$ defines a sequence of actions that is applicable with respect to $\{v\} \cup \operatorname{pred}(v)$. In addition, $\left|\rho_{v}^{*}\right|=\left|\rho \downarrow_{v}\right|$ together with Eq. 167 implies that this action sequence provides to $v$ the value $G[v]$ if the latter is specified.

(b) All the changes of $v$ to $\mathrm{b}_{v}$ are done using action $a_{1}^{\mathrm{b}}$, and then the largest possible number of changes to $\mathrm{w}_{v}$ are done using action $a_{1}^{\mathrm{w}}$, with the remaining changes to $\mathrm{w}_{v}$ being done using action $a_{2}^{\mathrm{w}}$. For this candidate for $\rho_{v}^{*}$, we set

$$
a_{i}^{*}= \begin{cases}a_{2}^{\mathrm{w}} & i=2 j-1, j \in \mathbb{N}, j \leq y-x \\ a_{1}^{\mathrm{w}} & i=2 j-1, j \in \mathbb{N}, y-x<j \leq y \\ a_{1}^{\mathrm{b}} & \text { otherwise }\end{cases}
$$

And the cost in this case is

$$
x \cdot \mathcal{C}\left(a_{1}^{\mathrm{w}}\right)+(y-x) \cdot \mathcal{C}\left(a_{2}^{\mathrm{w}}\right)+y \cdot \mathcal{C}\left(a_{1}^{\mathrm{b}}\right)
$$

The ordering constraints $>_{v}$ are set according to Eq. 75. Likewise, if $\rho_{w}^{*}=$ $\left\langle a_{1}, \ldots, a_{2 x-1}\right\rangle$, we set $>_{v, w}$ according to Eq. 159 above.

For each $u \in \operatorname{pred}(v) \backslash\{w\}$ we set $>_{v, u}$ according to Eq. 86 above.

The relations $>_{v},>_{v, w}$ and $>_{v, u}$ are effectively identical to the previous case. Thus, we have $>_{v} \cup>_{v, u}$ and $>_{v} \cup>_{v, w}$ forming strict partial orders over the unions of the elements of $\rho_{v}^{*}$ and $\rho_{u}^{*}$, and $\rho_{v}^{*}$ and $\rho_{w}^{*}$, respectively.

From Eqs. 75, 86, 159 we can now derive that any linearization of $>_{v} \cup \bigcup_{w \in \operatorname{pred}(v)}>_{v, w}$ defines a sequence of actions that is applicable with respect to $\{v\} \cup \operatorname{pred}(v)$. In addition, $\left|\rho_{v}^{*}\right|=\left|\rho \downarrow_{v}\right|$ together with Eq. 170 implies that this action sequence provides to $v$ the value $G[v]$ if the latter is specified.

Now, for each cost-optimal plan $\rho, \rho \downarrow_{v}$ cannot contain more than $y+x$ actions of both types $a_{1}^{\mathrm{w}}$ and $a_{1}^{\mathrm{b}}$ totally. Suppose to the contrary that $\rho \downarrow_{v}$ contain at least $y+x+1$ actions of types $a_{1}^{\mathrm{w}}$ and $a_{1}^{\mathrm{b}}$. Then it contains no more than $y-x-1$ actions of other types. Let $\mathbf{w}_{w} \cdot \mathbf{b}_{w} \cdot \ldots \cdot \mathbf{b}_{w}$ sequence of $2 y$ values of $w$ that support a cost-optimal plan for $v$ given that $w$ can change its value any number of times. Then each action of any other type will decrease the needed length of this sequence by at most 2 . Therefore at most $y-x-1$ actions of other type will decrease the length by at most $2 y-2 x-2$, and we are left with the sequence of length $\geq 2 y-(2 y-2 x-2)=2 x+2$. Therefore $\sigma_{w}$ cannot support more than $y+x$ actions of types $a_{1}^{\mathrm{w}}$ and $a_{1}^{\mathrm{b}}$. Now, suppose that in some given cost-optimal plan $\rho \downarrow_{v}$ for $v$ there are $\alpha$ actions of type $a_{1}^{\mathrm{w}}$ and $\beta$ actions of type $a_{1}^{\mathrm{b}}$. Then

$$
\alpha+\beta \leq y+x
$$


and

$$
\mathcal{C}\left(\rho \downarrow_{v}\right) \geq \alpha \cdot \mathcal{C}\left(a_{1}^{\mathrm{w}}\right)+(y-\alpha) \cdot \mathcal{C}\left(a_{2}^{\mathrm{w}}\right)+\beta \cdot \mathcal{C}\left(a_{1}^{\mathrm{b}}\right)+(y-\beta) \cdot \mathcal{C}\left(a_{2}^{\mathrm{b}}\right)
$$

For $(168) \leq(171)$, we have

$$
\mathcal{C}\left(a_{2}^{\mathrm{b}}\right)-\mathcal{C}\left(a_{1}^{\mathrm{b}}\right) \leq \mathcal{C}\left(a_{2}^{\mathrm{w}}\right)-\mathcal{C}\left(a_{1}^{\mathrm{w}}\right)
$$

Now suppose to the contrary that the plan in first case is not cost-optimal. Then from Eq. 173 we have

$$
\begin{aligned}
& \alpha \cdot \mathcal{C}\left(a_{1}^{\mathrm{w}}\right)+(y-\alpha) \cdot \mathcal{C}\left(a_{2}^{\mathrm{w}}\right)+\beta \cdot \mathcal{C}\left(a_{1}^{\mathrm{b}}\right)+(y-\beta) \cdot \mathcal{C}\left(a_{2}^{\mathrm{b}}\right)< \\
& y \cdot \mathcal{C}\left(a_{1}^{\mathrm{w}}\right)+x \cdot \mathcal{C}\left(a_{1}^{\mathrm{b}}\right)+(y-x) \cdot \mathcal{C}\left(a_{2}^{\mathrm{b}}\right)
\end{aligned}
$$

and from it

$$
(y-\alpha) \cdot\left(\mathcal{C}\left(a_{2}^{\mathrm{w}}\right)-\mathcal{C}\left(a_{1}^{\mathrm{w}}\right)\right)<(\beta-x) \cdot\left(\mathcal{C}\left(a_{2}^{\mathrm{b}}\right)-\mathcal{C}\left(a_{1}^{\mathrm{b}}\right)\right)
$$

From Eq. 172 we have $y-\alpha \geq \beta-x$, together with Eq. 174 contradicting Eq. 175 . For $(171) \leq(168)$, we have

$$
\mathcal{C}\left(a_{2}^{\mathrm{w}}\right)-\mathcal{C}\left(a_{1}^{\mathrm{w}}\right) \leq \mathcal{C}\left(a_{2}^{\mathrm{b}}\right)-\mathcal{C}\left(a_{1}^{\mathrm{b}}\right)
$$

Now suppose to the contrary that the plan in second case is not cost-optimal. Then from Eq. 173 we have

$$
\begin{aligned}
& \alpha \cdot \mathcal{C}\left(a_{1}^{\mathrm{w}}\right)+(y-\alpha) \cdot \mathcal{C}\left(a_{2}^{\mathrm{w}}\right)+\beta \cdot \mathcal{C}\left(a_{1}^{\mathrm{b}}\right)+(y-\beta) \cdot \mathcal{C}\left(a_{2}^{\mathrm{b}}\right)< \\
& x \cdot \mathcal{C}\left(a_{1}^{\mathrm{w}}\right)+(y-x) \cdot \mathcal{C}\left(a_{2}^{\mathrm{w}}\right)+y \cdot \mathcal{C}\left(a_{1}^{\mathrm{b}}\right)
\end{aligned}
$$

and from it

$$
(y-\beta) \cdot\left(\mathcal{C}\left(a_{2}^{\mathrm{b}}\right)-\mathcal{C}\left(a_{1}^{\mathrm{b}}\right)\right)<(\alpha-x) \cdot\left(\mathcal{C}\left(a_{2}^{\mathrm{w}}\right)-\mathcal{C}\left(a_{1}^{\mathrm{w}}\right)\right)
$$

From Eq. 172 we have $y-\beta \geq \alpha-x$, together with Eq. 176 contradicting Eq. 177. (8) If $\left|\rho \downarrow_{v}\right|=2 y,\left|\sigma_{w}\right|=2 x+1,\left|\sigma_{w}\right|>\left|\rho \downarrow_{v}\right|$, then the actions of $\rho_{v}^{*}$ are set to

$$
a_{i}^{*}= \begin{cases}a_{\mathrm{w}_{v} \mid \mathrm{w}_{w}} & i=2 j-1, j \in \mathbb{N} \\ a_{\mathrm{b}_{v} \mid \mathrm{b}_{w}} & \text { otherwise }\end{cases}
$$

The ordering constraints $>_{v}$ are set according to Eq. 75. Likewise, if $\rho_{w}^{*}=$ $\left\langle a_{1}, \ldots, a_{2 x-1}\right\rangle$, we set $>_{v, w}$ according to Eq. 132 above.

Finally, the ordering constraints $>_{v, w^{\prime}}$ for the rest of the parents $w^{\prime} \in \operatorname{pred}(v) \backslash$ $\{u, w\}$ are set to empty sets.

The relations $>_{v}$ and $>_{v, w}$ are identical to the previous case. Thus, we have $>_{v} \cup>_{v, w}$ forming strict partial order over the union of the elements of $\rho_{v}^{*}$ and $\rho_{w}^{*}$. 
Until now, we have specified the sequences $\rho_{v}^{*}$, the orders $>_{v}$ induced by these sequences, the orders $>_{v, w}$, and proved that all $>_{v} \cup>_{v, w}$ and $>_{w} \cup>_{v, w}$ form strict partial orders over their domains. This construction allows us to apply now Theorem 1 to the (considered as sets) sequences $\rho_{v}^{*}$ and orders $>_{v}$ and $>_{v, w}$, proving that

$$
>=\bigcup_{v \in V}\left(>_{v} \cup \bigcup_{w \in \operatorname{pred}(v)}>_{v, w}\right)
$$

forms a strict partial order over the union of $\rho_{v_{1}}^{*}, \ldots, \rho_{v_{n}}^{*}$. Putting thing together, the above implies that any linearization $\rho^{*}$ of $>$ is a plan for $\Pi$, and post-3/2ness of all its subsequences $\rho_{v_{1}}^{*}, \ldots, \rho_{v_{n}}^{*}$ then implies $\rho^{*} \in \mathcal{P}^{3 / 2}(\Pi)$. Moreover, if $\rho$ is an optimal plan for $\Pi$, then $\mathcal{C}\left(\rho^{*}\right)=\mathcal{C}(\rho)$ implies the optimality of $\rho^{*}$.

Theorem 9 Let $\Pi$ be a $\mathbf{P}(1)$ problem, $\operatorname{COP}_{\Pi}=(\mathcal{X}, \mathcal{F})$ be the corresponding constraint optimization problem, and $\bar{x}$ be an optimal assignment to $\mathcal{X}$ with $\sum_{\varphi \in \mathcal{F}} \varphi(\bar{x})=\alpha$.

(I) If $\alpha<\infty$, then a plan of cost $\alpha$ for $\Pi$ can be reconstructed from $\bar{x}$ in time polynomial in the description size of $\Pi$.

(II) If $\Pi$ has a plan, then $\alpha<\infty$.

\section{Proof:}

(I) Given a COP solution $\bar{x}$ with $\sum_{\varphi \in \mathcal{F}} \varphi(\bar{x})=\alpha<\infty$, we construct a plan $\rho$ for $\Pi$ with $\mathcal{C}(\rho)=\alpha$. We construct this plan by

1. Traversing the planning variables in a topological ordering of the causal graph $C G(\Pi)$, and associating each variable $v$ with a sequence $\rho_{v} \in A_{v}^{*}$.

2. Merging the constructed sequences $\rho_{v_{1}}, \ldots, \rho_{v_{n}}$ into the desired plan $\rho$.

For each $v \in V$ with $\operatorname{pred}(v)=\emptyset$ we set $\rho_{v}=\left\langle a_{1} \cdot \ldots \cdot a_{l}\right\rangle$, where $l=\left|\bar{x}_{v}\right|-1$, and $a_{i}$ is defined as in Eq 179 below.

$$
a_{i}= \begin{cases}a_{\mathrm{w}_{v}}, & i \text { is odd, } \\ a_{\mathbf{b}_{v}}, & i \text { is even, }\end{cases}
$$

Note that Eq. 179 is well-defined - the existence of the essential for Eq. 179 actions $a_{\mathrm{w}_{v}} / a_{\mathrm{b}_{v}}$ is implied by Eq. 22 and $\alpha<\infty$.

In turn, for each $v \in V$ with $\operatorname{pred}(v)=\left\{w_{1}, \ldots, w_{k}\right\}$, given $\bar{x}_{v}^{w_{1}}, \ldots, \bar{x}_{v}^{w_{k}}$, we distinguish between the following cases.

[ $\mathbf{R} 1$ is played ] $\mathrm{R} 1$ is played by one of the parents, while all other parents play role R11.

[ R1 is played by $w_{1}$ ] Eq. 35 then implies

$$
\varphi_{x_{v} w_{1}}\left(\bar{x}_{v}^{w_{1}}, \bar{x}_{w_{1}}\right)=\varphi\left(\mathrm{R} 1, \bar{x}_{v}^{w_{1}}, \bar{x}_{w_{1}}\right)
$$

and $\bar{x}_{v}^{w_{1}} \ni \mathbf{S}=\mathrm{S} 1$. From Eq. 36 we then have $\bar{x}_{v}^{w_{j}} \ni \mathbf{S}^{\prime}=\mathrm{S} 1$ for each $1<j \leq k$, giving us

$$
\varphi_{x_{v}}^{w_{j}}\left(\bar{x}_{v}^{w_{j}}, \bar{x}_{v}^{w_{j-1}}, \bar{x}_{w_{j}}\right)=0
$$


[ $\mathbf{R} 1$ is played by $w_{j}, j>1$ ] Eq. 36 then implies

$$
\begin{aligned}
\left.\varphi_{x_{v} w_{j}}\left(\llbracket \mathbf{S}, \#_{\mathrm{w}}, \#_{\mathrm{b}}, \eta\right], \llbracket \mathbf{S}^{\prime}, \#_{\mathrm{w}}^{\prime}, \#_{\mathrm{b}}^{\prime}, \eta^{\prime} \rrbracket, \sigma_{w_{j}}\right)= \\
\varphi\left(\mathrm{R} 1, \llbracket \mathbf{S}, \#_{\mathrm{w}}-\#_{\mathrm{w}}^{\prime}, \#_{\mathbf{b}}-\#_{\mathrm{b}}^{\prime}, \eta \rrbracket, \sigma_{w_{j}}\right)
\end{aligned}
$$

and, for all $1<i \neq j \leq k$,

$$
\varphi_{x_{v}^{w_{i}}}\left(\bar{x}_{v}^{w_{i}}, \bar{x}_{v}^{w_{i-1}}, \bar{x}_{w_{i}}\right)=0
$$

From Eq. 35 we also have

$$
\varphi_{x_{v}^{w_{1}}}\left(\bar{x}_{v}^{w_{1}}, \bar{x}_{w_{1}}\right)=0
$$

In both these sub-cases, $\rho_{v},>_{v}$ and $>_{v, w}$ are specified as in the proof of Theorem 8 , case I.

[ $\mathbf{R} 2$ is played ] R2 is played by one of the parents, while all other parents play role R11.

[ R2 is played by $w_{1}$ ] Eq. 35 then implies

$$
\varphi_{x_{v} w_{1}}\left(\bar{x}_{v}^{w_{1}}, \bar{x}_{w_{1}}\right)=\varphi\left(\mathrm{R} 2, \bar{x}_{v}^{w_{1}}, \bar{x}_{w_{1}}\right)
$$

and, for each $1<j \leq k$, Eq. 36 implies

$$
\varphi_{x_{v} w_{j}}\left(\bar{x}_{v}^{w_{j}}, \bar{x}_{v}^{w_{j-1}}, \bar{x}_{w_{j}}\right)=0
$$

If $\varphi\left(\mathrm{R} 2,\left[\mathbf{S}, \#_{\mathrm{w}}, \#_{\mathbf{b}}, \eta\right], \sigma_{w_{1}}\right)=\xi\left(\#_{\mathrm{w}}, 0, \#_{\mathbf{b}}, 0\right)$, then $\rho_{v},>_{v}$ and $>_{v, w}$ are specified as in the proof of Theorem 8, case III.2, otherwise, as in the case IV.2.

[ $\mathbf{R} 2$ is played by $w_{j}, j>1$ ] Eq. 36 then implies

$$
\begin{aligned}
& \varphi_{x_{v}}^{w_{j}}\left(\llbracket \mathbf{S}, \#_{\mathrm{w}}, \#_{\mathbf{b}}, \eta\right],\left.\llbracket \mathbf{S}^{\prime}, \#_{\mathbf{w}}^{\prime}, \#_{\mathbf{b}}^{\prime}, \eta^{\prime} \rrbracket, \sigma_{w_{j}}\right)= \\
& \varphi\left(\mathrm{R} 2, \llbracket \mathbf{S}, \#_{\mathrm{w}}-\#_{\mathrm{w}}^{\prime}, \#_{\mathbf{b}}-\#_{\mathbf{b}}^{\prime}, \eta \rrbracket, \sigma_{w_{j}}\right)
\end{aligned}
$$

and, for all $1<i \neq j \leq k$,

$$
\varphi_{x_{v}^{w_{i}}}\left(\bar{x}_{v}^{w_{i}}, \bar{x}_{v}^{w_{i-1}}, \bar{x}_{w_{i}}\right)=0
$$

From Eq. 35 we also have

$$
\varphi_{x_{v}^{w_{1}}}\left(\bar{x}_{v}^{w_{1}}, \bar{x}_{w_{1}}\right)=0
$$

If $\varphi\left(\mathrm{R} 2,\left[\mathbf{S}, \#_{\mathrm{w}}-\#_{\mathrm{w}}^{\prime}, \#_{\mathrm{b}}-\#_{\mathrm{b}}^{\prime}, \eta\right], \sigma_{w_{j}}\right)=\xi\left(\#_{\mathrm{w}}-\#_{\mathrm{w}}^{\prime}, 0, \#_{\mathrm{b}}-\#_{\mathrm{b}}^{\prime}, 0\right)$, then $\rho_{v},>_{v}$ and $>_{v, w}$ are specified as in the proof of Theorem 8, case III.2, otherwise, as in the case IV.2.

[ R3 and R4 are played ] Those roles are played by two of the parents, while all other parents play role $\mathrm{R} 11$. 
[ $\mathbf{R} 3$ is played by $w_{1}, \mathbf{R} 4$ is played by $w_{j}, j>1$ ] From Eqs. 35 and 36 we then have

$$
\varphi_{x_{v} w_{1}}\left(\bar{x}_{v}^{w_{1}}, \bar{x}_{w_{1}}\right)=\varphi\left(\mathrm{R} 3, \bar{x}_{v}^{w_{1}}, \bar{x}_{w_{1}}\right)
$$

and

$$
\begin{aligned}
& \varphi_{x_{v}}^{w_{j}}\left(\llbracket \mathbf{S}, \#_{\mathrm{w}}, \#_{\mathbf{b}}, \eta\right],\left.\llbracket \mathbf{S}^{\prime}, \#_{\mathrm{w}}^{\prime}, \#_{\mathbf{b}}^{\prime}, \eta^{\prime} \rrbracket, \sigma_{w_{j}}\right)= \\
& \varphi\left(\mathrm{R} 4, \llbracket \mathbf{S}, \#_{\mathrm{w}}-\#_{\mathrm{w}}^{\prime}, \#_{\mathbf{b}}-\#_{\mathbf{b}}^{\prime}, \eta \rrbracket, \sigma_{w_{j}}\right)
\end{aligned}
$$

and, for all $1<i \leq k$, such that $i \neq j$ :

$$
\varphi_{x_{v}^{w_{i}}}\left(\bar{x}_{v}^{w_{i}}, \bar{x}_{v}^{w_{i-1}}, \bar{x}_{w_{i}}\right)=0
$$

[ $\mathbf{R} 4$ is played by $w_{1}, \mathbf{R} 3$ is played by $w_{j}, j>1$ ] From Eqs. 35 and 36 we then have

$$
\varphi_{x_{v} w_{1}}\left(\bar{x}_{v}^{w_{1}}, \bar{x}_{w_{1}}\right)=\varphi\left(\mathrm{R} 4, \bar{x}_{v}^{w_{1}}, \bar{x}_{w_{1}}\right)
$$

and

$$
\begin{aligned}
& \varphi_{x_{v}}^{w_{j}}\left(\llbracket \mathbf{S}, \#_{\mathrm{w}}, \#_{\mathbf{b}}, \eta\right],\left.\llbracket \mathbf{S}^{\prime}, \#_{\mathbf{w}}^{\prime}, \#_{\mathbf{b}}^{\prime}, \eta^{\prime} \rrbracket, \sigma_{w_{j}}\right)= \\
& \varphi\left(\mathrm{R} 3, \llbracket \mathbf{S}, \#_{\mathrm{w}}-\#_{\mathrm{w}}^{\prime}, \#_{\mathbf{b}}-\#_{\mathbf{b}}^{\prime}, \eta \rrbracket, \sigma_{w_{j}}\right)
\end{aligned}
$$

and, for all $1<i \neq j \leq k$,

$$
\varphi_{x_{v}^{w_{i}}}\left(\bar{x}_{v}^{w_{i}}, \bar{x}_{v}^{w_{i-1}}, \bar{x}_{w_{i}}\right)=0
$$

[ $\mathbf{R} 3$ is played by $w_{j}, \mathbf{R} 4$ is played by $w_{t}, j \neq t, j, t>1$ ] From Eqs. 35 and 36 we have

$$
\varphi_{x_{v}^{w_{1}}}\left(\bar{x}_{v}^{w_{1}}, \bar{x}_{w_{1}}\right)=0
$$

and

$$
\begin{aligned}
& \varphi_{x_{v}}^{w_{j}}\left(\llbracket \mathbf{S}, \#_{\mathrm{w}}, \#_{\mathbf{b}}, \eta\right],\left.\llbracket \mathbf{S}^{\prime}, \#_{\mathrm{w}}^{\prime}, \#_{\mathrm{b}}^{\prime}, \eta^{\prime} \rrbracket, \sigma_{w_{j}}\right)= \\
& \varphi\left(\mathrm{R} 3, \llbracket \mathbf{S}, \#_{\mathrm{w}}-\#_{\mathrm{w}}^{\prime}, \#_{\mathbf{b}}-\#_{\mathbf{b}}^{\prime}, \eta \rrbracket, \sigma_{w_{j}}\right)
\end{aligned}
$$

and

$$
\begin{aligned}
\varphi_{x_{v} w_{t}}\left(\llbracket \mathbf{S}, \#_{\mathrm{w}}, \#_{\mathrm{b}}, \eta\right], & \left.\llbracket \mathbf{S}^{\prime}, \#_{\mathrm{w}}^{\prime}, \#_{\mathrm{b}}^{\prime}, \eta^{\prime} \rrbracket, \sigma_{w_{t}}\right)= \\
& \varphi\left(\mathrm{R} 4, \llbracket \mathbf{S}, \#_{\mathrm{w}}-\#_{\mathrm{w}}^{\prime}, \#_{\mathrm{b}}-\#_{\mathrm{b}}^{\prime}, \eta \rrbracket, \sigma_{w_{t}}\right)
\end{aligned}
$$

and, for all $1<i \leq k$ such that $i \notin\{j, t\}$,

$$
\varphi_{x_{v}^{w_{i}}}\left(\bar{x}_{v}^{w_{i}}, \bar{x}_{v}^{w_{i-1}}, \bar{x}_{w_{i}}\right)=0
$$

In all these three sub-cases, $\rho_{v},>_{v}$ and $>_{v, w}$ are specified as in the proof of Theorem 8 , case II.

[ $\mathbf{R} 5$ is played ] $\mathrm{R} 5$ is played by one of the parents, while all other parents play role R11. 
[ R5 is played by $w_{1}$ ] Eqs. 35 and 36 imply

$$
\varphi_{x_{v} w_{1}}\left(\bar{x}_{v}^{w_{1}}, \bar{x}_{w_{1}}\right)=\varphi\left(\mathrm{R} 5, \bar{x}_{v}^{w_{1}}, \bar{x}_{w_{1}}\right)
$$

and, for each $1<j \leq k$,

$$
\varphi_{x_{v} w_{j}}\left(\bar{x}_{v}^{w_{j}}, \bar{x}_{v}^{w_{j-1}}, \bar{x}_{w_{j}}\right)=0
$$

Considering now the specification of the function $\varphi$ in Eq. 29,

- If the first case holds, and the minimum is obtained at the first expression, then $\rho_{v},>_{v}$ and $>_{v, w}$ are defined as in the proof of Theorem 8, case III.1.a.

- If the first case holds, and the minimum is obtained at the second expression, then $\rho_{v},>_{v}$ and $>_{v, w}$ are defined as in the proof of Theorem 8, case IV.1.a.

- If the second case holds, and the minimum is obtained at the first expression, then $\rho_{v},>_{v}$ and $>_{v, w}$ are defined as in the proof of Theorem 8, case III.3.a.

- If the second case holds, and the minimum is obtained at the second expression, then $\rho_{v},>_{v}$ and $>_{v, w}$ are defined as in the proof of Theorem 8, case IV.3.a.

- If the third case holds, then $\rho_{v},>_{v}$ and $>_{v, w}$ are defined as in the proof of Theorem 8, case III.3.a.

- If the forth case holds, then $\rho_{v},>_{v}$ and $>_{v, w}$ are defined as in the proof of Theorem 8, case IV.3.a.

- If the fifth case holds, and the minimum is obtained at the first expression, then $\rho_{v},>_{v}$ and $>_{v, w}$ are defined as in the proof of Theorem 8, case III.5.a.

- If the fifth case holds, and the minimum is obtained at the second expression, then $\rho_{v},>_{v}$ and $>_{v, w}$ are defined as in the proof of Theorem 8, case IV.5.a.

- If the sixth case holds, then $\rho_{v},>_{v}$ and $>_{v, w}$ are defined as in the proof of Theorem 8, case III.5.a.

- If the seventh case holds, then $\rho_{v},>_{v}$ and $>_{v, w}$ are defined as in the proof of Theorem 8, case IV.5.a.

- If the eighth case holds, and the minimum is obtained at the first expression, then $\rho_{v},>_{v}$ and $>_{v, w}$ are defined as in the proof of Theorem 8, case III.7.a.

- If the eighth case holds, and the minimum is obtained at the second expression, then $\rho_{v},>_{v}$ and $>_{v, w}$ are defined as in the proof of Theorem 8, case IV.7.a.

[ R5 is played by $w_{j}, j>1$ ] Eq. 36 then implies

$$
\begin{aligned}
\left.\varphi_{x_{v}}^{w_{j}}\left(\llbracket \mathbf{S}, \#_{\mathrm{w}}, \#_{\mathbf{b}}, \eta\right], \llbracket \mathbf{S}^{\prime}, \#_{\mathrm{w}}^{\prime}, \#_{\mathrm{b}}^{\prime}, \eta^{\prime} \rrbracket, \sigma_{w_{j}}\right)= \\
\varphi\left(\mathrm{R} 5, \llbracket \mathbf{S}, \#_{\mathrm{w}}-\#_{\mathrm{w}}^{\prime}, \#_{\mathbf{b}}-\#_{\mathbf{b}}^{\prime}, \eta \rrbracket, \sigma_{w_{j}}\right)
\end{aligned}
$$

and, for all $1<i \neq j \leq k$,

$$
\varphi_{x_{v}}^{w_{i}}\left(\bar{x}_{v}^{w_{i}}, \bar{x}_{v}^{w_{i-1}}, \bar{x}_{w_{i}}\right)=0
$$

From Eq. 35 we also have

$$
\varphi_{x_{v}^{w_{1}}}\left(\bar{x}_{v}^{w_{1}}, \bar{x}_{w_{1}}\right)=0
$$

Here $\rho_{v},>_{v}$ and $>_{v, w}$ are specified exactly as in the previous case. 
[ $\mathbf{R} 6$ is played ] R6 is played by one of the parents, while all other parents play role R11.

[ $\mathbf{R} 6$ is played by $w_{1}$ ] Eqs. 35 and 36 imply

$$
\varphi_{x_{v} w_{1}}\left(\bar{x}_{v}^{w_{1}}, \bar{x}_{w_{1}}\right)=\varphi\left(\mathrm{R} 6, \bar{x}_{v}^{w_{1}}, \bar{x}_{w_{1}}\right)
$$

and, for each $1<j \leq k$,

$$
\varphi_{x_{v} w_{j}}\left(\bar{x}_{v}^{w_{j}}, \bar{x}_{v}^{w_{j-1}}, \bar{x}_{w_{j}}\right)=0
$$

Considering now the specification of the function $\varphi$ in Eq. 30,

- If the first case holds, and the minimum is obtained at the first expression, then $\rho_{v},>_{v}$ and $>_{v, w}$ are defined as in the proof of Theorem 8, case III.1.b.

- If the first case holds, and the minimum is obtained at the second expression, then $\rho_{v},>_{v}$ and $>_{v, w}$ are defined as in the proof of Theorem 8, case IV.1.b.

- If the second case holds, and the minimum is obtained at the first expression, then $\rho_{v},>_{v}$ and $>_{v, w}$ are defined as in the proof of Theorem 8, case III.3.b.

- If the second case holds, and the minimum is obtained at the second expression, then $\rho_{v},>_{v}$ and $>_{v, w}$ are defined as in the proof of Theorem 8, case IV.3.b.

- If the third case holds, then $\rho_{v},>_{v}$ and $>_{v, w}$ are defined as in the proof of Theorem 8, case III.3.b.

- If the forth case holds, then $\rho_{v},>_{v}$ and $>_{v, w}$ are defined as in the proof of Theorem 8, case IV.3.b.

- If the fifth case holds, and the minimum is obtained at the first expression, then $\rho_{v},>_{v}$ and $>_{v, w}$ are defined as in the proof of Theorem 8, case III.5.b.

- If the fifth case holds, and the minimum is obtained at the second expression, then $\rho_{v},>_{v}$ and $>_{v, w}$ are defined as in the proof of Theorem 8, case IV.5.b.

- If the sixth case holds, then $\rho_{v},>_{v}$ and $>_{v, w}$ are defined as in the proof of Theorem 8, case III.5.b.

- If the seventh case holds, and the minimum is obtained at the first expression, then $\rho_{v},>_{v}$ and $>_{v, w}$ are defined as in the proof of Theorem 8, case III.7.b.

- If the seventh case holds, and the minimum is obtained at the second expression, then $\rho_{v},>_{v}$ and $>_{v, w}$ are defined as in the proof of Theorem 8, case IV.7.b.

[ $\mathbf{R} 6$ is played by $w_{j}, j>1$ ] Eq. 36 then implies

$$
\begin{aligned}
& \varphi_{x_{v}}^{w_{j}}\left(\llbracket \mathbf{S}, \#_{\mathrm{w}}, \#_{\mathbf{b}}, \eta\right],\left.\llbracket \mathbf{S}^{\prime}, \#_{\mathrm{w}}^{\prime}, \#_{\mathrm{b}}^{\prime}, \eta^{\prime} \rrbracket, \sigma_{w_{j}}\right)= \\
& \varphi\left(\mathrm{R} 6, \llbracket \mathbf{S}, \#_{\mathrm{w}}-\#_{\mathrm{w}}^{\prime}, \#_{\mathbf{b}}-\#_{\mathrm{b}}^{\prime}, \eta \rrbracket, \sigma_{w_{j}}\right)
\end{aligned}
$$

and, for all $1<i \neq j \leq k$,

$$
\varphi_{x_{v} w_{i}}\left(\bar{x}_{v}^{w_{i}}, \bar{x}_{v}^{w_{i-1}}, \bar{x}_{w_{i}}\right)=0
$$

From Eq. 35 we also have

$$
\varphi_{x_{v} w_{1}}\left(\bar{x}_{v}^{w_{1}}, \bar{x}_{w_{1}}\right)=0
$$

Here $\rho_{v},>_{v}$ and $>_{v, w}$ are specified exactly as in the previous case. 
[ R7 and R9 are played ] Those roles are played by two of the parents, while all other parents play role R11.

[ $\mathbf{R} 7$ is played by $w_{1}, \mathbf{R} 9$ is played by $w_{j}, j>1$ ] From Eqs. 35 and 36 we then have

$$
\varphi_{x_{v} w_{1}}\left(\bar{x}_{v}^{w_{1}}, \bar{x}_{w_{1}}\right)=\varphi\left(\mathrm{R} 7, \bar{x}_{v}^{w_{1}}, \bar{x}_{w_{1}}\right)
$$

and

$$
\begin{aligned}
\left.\varphi_{x_{v}}^{w_{j}}\left(\llbracket \mathbf{S}, \#_{\mathrm{w}}, \#_{\mathbf{b}}, \eta\right], \llbracket \mathbf{S}^{\prime}, \#_{\mathrm{w}}^{\prime}, \#_{\mathbf{b}}^{\prime}, \eta^{\prime} \rrbracket, \sigma_{w_{j}}\right)= \\
\varphi\left(\mathrm{R} 9, \llbracket \mathbf{S}, \#_{\mathrm{w}}-\#_{\mathrm{w}}^{\prime}, \#_{\mathbf{b}}-\#_{\mathbf{b}}^{\prime}, \eta \rrbracket, \sigma_{w_{j}}\right)
\end{aligned}
$$

and ,for all $1<i \neq j \leq k$,

$$
\varphi_{x_{v}^{w_{i}}}\left(\bar{x}_{v}^{w_{i}}, \bar{x}_{v}^{w_{i-1}}, \bar{x}_{w_{i}}\right)=0
$$

Considering now the specification of the function $\varphi$ in Eq. 31,

- If the first case holds, and the minimum is obtained at the first expression, then $\rho_{v},>_{v}$ and $>_{v, w}$ are defined as in the proof of Theorem 8, case III.1.a.

- If the first case holds, and the minimum is obtained at the second expression, then $\rho_{v},>_{v}$ and $>_{v, w}$ are defined as in the proof of Theorem 8, case IV.1.a.

- If the second case holds, then $\rho_{v},>_{v}$ and $>_{v, w}$ are defined as in the proof of Theorem 8, case III.3.a.

- If the third case holds, then $\rho_{v},>_{v}$ and $>_{v, w}$ are defined as in the proof of Theorem 8, case IV.3.a.

- If the forth case holds, then $\rho_{v},>_{v}$ and $>_{v, w}$ are defined as in the proof of Theorem 8, case III.5.a.

- If the fifth case holds, then $\rho_{v},>_{v}$ and $>_{v, w}$ are defined as in the proof of Theorem 8, case IV.5.a.

- If the sixth case holds, and the minimum is obtained at the first expression, then $\rho_{v},>_{v}$ and $>_{v, w}$ are defined as in the proof of Theorem 8, case III.7.a.

- If the sixth case holds, and the minimum is obtained at the second expression, then $\rho_{v},>_{v}$ and $>_{v, w}$ are defined as in the proof of Theorem 8, case IV.7.a.

[ $\mathbf{R} 9$ is played by $w_{1}, \mathbf{R} 7$ is played by $w_{j}, j>1$ ] From Eqs. 35 and 36 we then have

$$
\varphi_{x_{v} w_{1}}\left(\bar{x}_{v}^{w_{1}}, \bar{x}_{w_{1}}\right)=\varphi\left(\mathrm{R} 9, \bar{x}_{v}^{w_{1}}, \bar{x}_{w_{1}}\right)
$$

and

$$
\begin{aligned}
& \varphi_{x_{v} w_{j}}\left(\llbracket \mathbf{S}, \#_{\mathrm{w}}, \#_{\mathbf{b}}, \eta\right],\left.\llbracket \mathbf{S}^{\prime}, \#_{\mathrm{w}}^{\prime}, \#_{\mathbf{b}}^{\prime}, \eta^{\prime} \rrbracket, \sigma_{w_{j}}\right)= \\
& \varphi\left(\mathrm{R} 7, \llbracket \mathbf{S}, \#_{\mathrm{w}}-\#_{\mathrm{w}}^{\prime}, \#_{\mathbf{b}}-\#_{\mathbf{b}}^{\prime}, \eta \rrbracket, \sigma_{w_{j}}\right)
\end{aligned}
$$

and, for all $1<i \neq j \leq k$,

$$
\varphi_{x_{v}^{w_{i}}}\left(\bar{x}_{v}^{w_{i}}, \bar{x}_{v}^{w_{i-1}}, \bar{x}_{w_{i}}\right)=0
$$

Here $\rho_{v},>_{v}$ and $>_{v, w}$ are specified exactly as in the previous case. 
[ $\mathbf{R} 7$ is played by $w_{j}, \mathbf{R} 9$ is played by $w_{t}, j \neq t, j, t>1$ ] From Eqs. 35 and 36 we have

$$
\varphi_{x_{v}^{w_{1}}}\left(\bar{x}_{v}^{w_{1}}, \bar{x}_{w_{1}}\right)=0
$$

and

$$
\begin{aligned}
\left.\varphi_{x_{v}}^{w_{j}}\left(\llbracket \mathbf{S}, \#_{\mathrm{w}}, \#_{\mathbf{b}}, \eta\right], \llbracket \mathbf{S}^{\prime}, \#_{\mathrm{w}}^{\prime}, \#_{\mathbf{b}}^{\prime}, \eta^{\prime} \rrbracket, \sigma_{w_{j}}\right)= \\
\varphi\left(\mathrm{R} 7, \llbracket \mathbf{S}, \#_{\mathrm{w}}-\#_{\mathrm{w}}^{\prime}, \#_{\mathbf{b}}-\#_{\mathbf{b}}^{\prime}, \eta \rrbracket, \sigma_{w_{j}}\right)
\end{aligned}
$$

and

$$
\begin{array}{r}
\varphi_{x_{v}^{w_{t}}}\left(\left[\mathbf{S}, \#_{\mathrm{w}}, \#_{\mathrm{b}}, \eta\right], \llbracket \mathbf{S}^{\prime}, \#_{\mathrm{w}}^{\prime}, \#_{\mathrm{b}}^{\prime}, \eta^{\prime} \rrbracket, \sigma_{w_{t}}\right)= \\
\varphi\left(\mathrm{R} 9, \llbracket \mathbf{S}, \#_{\mathrm{w}}-\#_{\mathrm{w}}^{\prime}, \#_{\mathrm{b}}-\#_{\mathrm{b}}^{\prime}, \eta \rrbracket, \sigma_{w_{t}}\right)
\end{array}
$$

and, for all $1<i \leq k$, such that $i \notin\{j, t\}$,

$$
\varphi_{x_{v}^{w_{i}}}\left(\bar{x}_{v}^{w_{i}}, \bar{x}_{v}^{w_{i-1}}, \bar{x}_{w_{i}}\right)=0
$$

Then, $\rho_{v},>_{v}$ and $>_{v, w}$ are specified exactly as in the two previous cases.

[ R8 and R10 are played ] Those roles are played by two of the parents, while all other parents play role R11.

[ $\mathbf{R} 8$ is played by $w_{1}, \mathbf{R} 10$ is played by $w_{j}, j>1$ ] From Eqs. 35 and 36 we then have

$$
\varphi_{x_{v} w_{1}}\left(\bar{x}_{v}^{w_{1}}, \bar{x}_{w_{1}}\right)=\varphi\left(\mathrm{R} 8, \bar{x}_{v}^{w_{1}}, \bar{x}_{w_{1}}\right)
$$

and

$$
\begin{aligned}
\varphi_{x_{v} w_{j}}\left(\left[\mathbf{S}, \#_{\mathrm{w}}, \#_{\mathbf{b}}, \eta \rrbracket,\right.\right. & \left.\llbracket \mathbf{S}^{\prime}, \#_{\mathrm{w}}^{\prime}, \#_{\mathrm{b}}^{\prime}, \eta^{\prime} \rrbracket, \sigma_{w_{j}}\right)= \\
& \varphi\left(\mathrm{R} 10, \llbracket \mathbf{S}, \#_{\mathrm{w}}-\#_{\mathrm{w}}^{\prime}, \#_{\mathbf{b}}-\#_{\mathbf{b}}^{\prime}, \eta \rrbracket, \sigma_{w_{j}}\right)
\end{aligned}
$$

and, for all $1<i \neq j \leq k$,

$$
\varphi_{x_{v}^{w_{i}}}\left(\bar{x}_{v}^{w_{i}}, \bar{x}_{v}^{w_{i-1}}, \bar{x}_{w_{i}}\right)=0
$$

Considering now the specification of the function $\varphi$ in Eq. 32,

- If the first case holds, and the minimum is obtained at the first expression, then $\rho_{v},>_{v}$ and $>_{v, w}$ are defined as in the proof of Theorem 8, case III.1.b.

- If the first case holds, and the minimum is obtained at the second expression, then $\rho_{v},>_{v}$ and $>_{v, w}$ are defined as in the proof of Theorem 8, case IV.1.b.

- If the second case holds, then $\rho_{v},>_{v}$ and $>_{v, w}$ are defined as in the proof of Theorem 8, case III.3.b.

- If the third case holds, then $\rho_{v},>_{v}$ and $>_{v, w}$ are defined as in the proof of Theorem 8, case IV.3.b.

- If the forth case holds, then $\rho_{v},>_{v}$ and $>_{v, w}$ are defined as in the proof of Theorem 8, case III.5.b.

- If the fifth case holds, then $\rho_{v},>_{v}$ and $>_{v, w}$ are defined as in the proof of Theorem 8, case IV.5.b. 
- If the sixth case holds, and the minimum is obtained at the first expression, then $\rho_{v},>_{v}$ and $>_{v, w}$ are defined as in the proof of Theorem 8, case III.7.b.

- If the sixth case holds, and the minimum is obtained at the second expression, then $\rho_{v},>_{v}$ and $>_{v, w}$ are defined as in the proof of Theorem 8, case IV.7.b.

[ $\mathbf{R} 10$ is played by $w_{1}, \mathbf{R} 8$ is played by $w_{j}, j>1$ ] From Eqs. 35 and 36 we then have

$$
\varphi_{x_{v}^{w_{1}}}\left(\bar{x}_{v}^{w_{1}}, \bar{x}_{w_{1}}\right)=\varphi\left(\mathrm{R} 10, \bar{x}_{v}^{w_{1}}, \bar{x}_{w_{1}}\right)
$$

and

$$
\begin{aligned}
& \varphi_{x_{v} w_{j}}\left(\llbracket \mathbf{S}, \#_{\mathrm{w}}, \#_{\mathbf{b}}, \eta\right],\left.\llbracket \mathbf{S}^{\prime}, \#_{\mathrm{w}}^{\prime}, \#_{\mathbf{b}}^{\prime}, \eta^{\prime} \rrbracket, \sigma_{w_{j}}\right)= \\
& \varphi\left(\mathrm{R} 8, \llbracket \mathbf{S}, \#_{\mathrm{w}}-\#_{\mathrm{w}}^{\prime}, \#_{\mathbf{b}}-\#_{\mathbf{b}}^{\prime}, \eta \rrbracket, \sigma_{w_{j}}\right)
\end{aligned}
$$

and, for all $1<i \neq j \leq k$,

$$
\varphi_{x_{v}^{w_{i}}}\left(\bar{x}_{v}^{w_{i}}, \bar{x}_{v}^{w_{i-1}}, \bar{x}_{w_{i}}\right)=0
$$

Here $\rho_{v},>_{v}$ and $>_{v, w}$ are specified exactly as in the previous case.

[ $\mathbf{R} 8$ is played by $w_{j}, \mathbf{R} 10$ is played by $w_{t}, j \neq t, j, t>1$ ] From Eqs. 35 and 36 we have

$$
\varphi_{x_{v}^{w_{1}}}\left(\bar{x}_{v}^{w_{1}}, \bar{x}_{w_{1}}\right)=0
$$

and

$$
\begin{aligned}
\left.\varphi_{x_{v}}^{w_{j}}\left(\llbracket \mathbf{S}, \#_{\mathrm{w}}, \#_{\mathbf{b}}, \eta\right], \llbracket \mathbf{S}^{\prime}, \#_{\mathrm{w}}^{\prime}, \#_{\mathbf{b}}^{\prime}, \eta^{\prime} \rrbracket, \sigma_{w_{j}}\right)= \\
\varphi\left(\mathrm{R} 8, \llbracket \mathbf{S}, \#_{\mathrm{w}}-\#_{\mathrm{w}}^{\prime}, \#_{\mathbf{b}}-\#_{\mathbf{b}}^{\prime}, \eta \rrbracket, \sigma_{w_{j}}\right)
\end{aligned}
$$

and

$$
\begin{array}{r}
\varphi_{x_{v}^{w_{t}}}\left(\left[\mathbf{S}, \#_{\mathrm{w}}, \#_{\mathrm{b}}, \eta\right], \llbracket \mathbf{S}^{\prime}, \#_{\mathrm{w}}^{\prime}, \#_{\mathrm{b}}^{\prime}, \eta^{\prime} \rrbracket, \sigma_{w_{t}}\right)= \\
\varphi\left(\mathrm{R} 10, \llbracket \mathbf{S}, \#_{\mathrm{w}}-\#_{\mathrm{w}}^{\prime}, \#_{\mathrm{b}}-\#_{\mathrm{b}}^{\prime}, \eta \rrbracket, \sigma_{w_{t}}\right)
\end{array}
$$

and, for all $1<i \leq k$, such that $i \notin\{j, t\}$,

$$
\varphi_{x_{v}^{w_{i}}}\left(\bar{x}_{v}^{w_{i}}, \bar{x}_{v}^{w_{i-1}}, \bar{x}_{w_{i}}\right)=0
$$

Then, $\rho_{v},>_{v}$ and $>_{v, w}$ are specified exactly as in the two previous cases.

Until now, for each variable $v \in V$, we have specified the action sequence $\rho_{v}$ and the order $>_{v}$ over the elements of $\rho_{v}$. For each $w \in \operatorname{pred}(v)$, we have specified the order $>_{v, w}$, and proved that all $>_{v} \cup>_{v, w}$ and $>_{w} \cup>_{v, w}$ form strict partial orders over their domains. Similarly to the uniform cost case, this construction allows us to apply now Theorem 1 on the (considered as sets) sequences $\rho_{v}$ and orders $>_{v}$ and $>_{v, w}$, proving that

$$
>=\bigcup_{v \in V}\left(>_{v} \cup \bigcup_{w \in \operatorname{pred}(v)}>_{v, w}\right)
$$

forms a strict partial order over the union of $\rho_{v_{1}}, \ldots, \rho_{v_{n}}$. 
Finally, we note that the plan extraction step of the algorithm polytree-1-dep corresponds exactly to the above construction along Eqs. 74-79, 81-83, 85-86, 93-94, 9697, 105-106, 108, 110, 117-118, 120, 122, 129-130, 132-133, 135, 142-143, 145, 153-154, 156$157,159,166-167,169-170,178$, providing us in poly-time with concrete cost-optimal plan corresponding to the optimal solution for $\mathrm{COP}_{\Pi}$.

(II) We now prove that if $\Pi$ is solvable, then we must have $\alpha<\infty$. Assume to the contrary that this is not the case. Let $\Pi$ be a solvable $\mathbf{P}(1)$ problem, and let (using Theorem 8) $\rho$ be an irreducible, post-3/2 plan for $\Pi$. Given such $\rho$, let a COP assignment $\bar{x}_{\rho}$ be defined as follows.

1. For each COP variable $x_{v}$, the assignment $\bar{x}_{\rho}$ provides the value $\sigma_{v} \in \unrhd^{*}[\sigma(v)]$ such that $\left|\sigma_{v}\right|=\left|\rho \downarrow_{v}\right|+1$.

2. For each variable $v \in V$, such that $\operatorname{pred}(v) \neq \emptyset$, find the (at most two) parents that prevail the actions in $\rho \downarrow_{v}$. Let $w$ be such a parent that performs a role $\mathrm{R} \in$ $\{\mathrm{R} 1, \mathrm{R} 2, \mathrm{R} 3, \mathrm{R} 5, \mathrm{R} 6, \mathrm{R} 7, \mathrm{R} 8\}$, and $w^{\prime}$ be the other such parent that performs one of the roles $\mathrm{R}^{\prime} \in\{\mathrm{R} 4, \mathrm{R} 9, \mathrm{R} 10, \mathrm{R} 11\}$. (By definition of post-3/2 action sequences, the rest of the parents all perform role R11.) Given that, if $|\operatorname{pred}(v)|=k>0$, we adopt an ordering of $\operatorname{pred}(v)$ such that $w_{1}=w$ and $w_{k}=w^{\prime}$. First, the assignment $\bar{x}_{v}^{w_{k}}$ to COP variable $x_{v}^{w_{k}}$ provides the value $\llbracket \mathrm{S} 1,\left\lceil\frac{\left|\sigma_{v}\right|-1}{2}\right\rceil,\left\lfloor\frac{\left|\sigma_{v}\right|-1}{2}\right\rfloor,\left|\sigma_{v}\right|-1 \rrbracket$. Then, for $1 \leq i<k$, the assignment $\bar{x}_{v}^{w_{i}}$ to COP variable $x_{v}^{w_{i}}$ provides the value $\left[\mathrm{S}, \#_{\mathrm{w}}, \#_{\mathrm{b}},\left|\sigma_{v}\right|-1\right]$, where

$$
\mathrm{S}= \begin{cases}\mathrm{S} 2, & \mathrm{R}^{\prime}=\mathrm{R} 4 \\ \mathrm{~S} 4, & \mathrm{R}^{\prime}=\mathrm{R} 9 \\ \mathrm{~S} 5, & \mathrm{R}^{\prime}=\mathrm{R} 10 \\ \mathrm{~S} 1, & \mathrm{R}^{\prime}=\mathrm{R} 11\end{cases}
$$

and $\#_{\mathrm{w}}$ and $\#_{\mathrm{b}}$ are the numbers of actions in $\rho \downarrow_{v}$ that change the value of $v$ to $\mathrm{w}_{v}$ and $\mathbf{b}_{v}$, respectively, while being prevailed by the value of $w_{1}$.

From Eq. 22-36 we then have that, for each $v \in V$, if $\operatorname{pred}(v)=\emptyset$, then $\varphi_{x_{v}}\left(\bar{x}_{v}\right)=\mathcal{C}\left(\rho \downarrow_{v}\right)$. Otherwise, if $\operatorname{pred}(v)=\left\{w_{1}, \ldots, w_{k}\right\}$, then $\varphi_{x_{v}}\left(\bar{x}_{v}, \bar{x}_{v}^{w_{k}}\right)=0$, and $\sum_{w \in \operatorname{pred}(v)} \varphi_{x_{v}^{w}}\left(\bar{x}_{\rho}\right)=$ $\mathcal{C}\left(\rho \downarrow_{v}\right)$. Therefore, we have

$$
\sum_{\varphi \in \mathcal{F}} \varphi\left(\bar{x}_{\rho}\right)=\sum_{v \in V} \mathcal{C}\left(\rho \downarrow_{v}\right)=\mathcal{C}(\rho)
$$

which is what we had to prove.

\section{References}

Bacchus, F., \& Yang, Q. (1994). Downward refinement and the efficiency of hierarchical problem solving. Artificial Intelligence, 71 (1), 43-100.

Bäckström, C., \& Klein, I. (1991). Planning in polynomial time: The SAS-PUBS class. Computational Intelligence, 7(3), 181-197. 
Bäckström, C., \& Nebel, B. (1995). Complexity results for $\mathrm{SAS}^{+}$planning. Computational Intelligence, 11(4), 625-655.

Bonet, B., \& Geffner, H. (2001). Planning as heuristic search. Artificial Intelligence, 129(12), 5-33.

Boutilier, C., Brafman, R., Domshlak, C., Hoos, H., \& Poole, D. (2004). CP-nets: A tool for representing and reasoning about conditional ceteris paribus preference statements. Journal of Artificial Intelligence Research, 21, 135-191.

Brafman, R. I., \& Domshlak, C. (2003). Structure and complexity of planning with unary operators. Journal of Artificial Intelligence Research, 18, 315-349.

Brafman, R. I., \& Domshlak, C. (2006). Factored planning: How, when, and when not. In Proceedings of the 18th National Conference on Artificial Intelligence (AAAI), pp. 809-814, Boston, MA.

Bylander, T. (1994). The computational complexity of propositional STRIPS planning. Artificial Intelligence, 69(1-2), 165-204.

Chapman, D. (1987). Planning for conjunctive goals. Artificial Intelligence, 32(3), 333-377.

Cormen, T. H., Leiserson, C. E., \& Rivest, R. L. (1990). Introduction to Algorithms. MIT Press.

Dechter, R. (2003). Constraint Processing. Morgan Kaufmann.

Edelkamp, S. (2001). Planning with pattern databases. In Proceedings of the European Conference on Planning (ECP), pp. 13-34.

Erol, K., Nau, D. S., \& Subrahmanian, V. S. (1995). Complexity, decidability and undecidability results for domain-independent planning. Artificial Intelligence, Special Issue on Planning, 76 (1-2), 75-88.

Garey, M. R., \& Johnson, D. S. (1978). Computers and Intractability: A Guide to the Theory of NP-Completeness. W.H. Freeman and Company, New-York.

Gimenez, O., \& Jonsson, A. (2008). The complexity of planning problems with simple causal graphs. Journal of Artificial Intelligence Research, 31, 319-351.

Haslum, P. (2006). Admissible Heuristics for Automated Planning. Ph.D. thesis, Linköping University, Department of Computer and Information Science.

Haslum, P., Bonet, B., \& Geffner, H. (2005). New admissible heuristics for domainindependent planning. In Proceedings of the 20th National Conference on Artificial Intelligence (AAAI), pp. 1163-1168, Pittsburgh, PA.

Haslum, P., \& Geffner, H. (2000). Admissible heuristics for optimal planning. In Proceedings of the 15th International Conference on Artificial Intelligence Planning Systems (AIPS), pp. 140-149, Breckenridge, CO.

Helmert, M. (2003). Complexity results for standard benchmark domains in planning. Artificial Intelligence, 146(2), 219-262.

Helmert, M. (2006). The Fast Downward planning system. Journal of Artificial Intelligence Research, 26, 191-246. 
Hoffmann, J. (2003). Utilizing Problem Structure in Planning: A Local Search Approach. No. 2854 in LNAI. Springer-Verlag.

Hoffmann, J., \& Edelkamp, S. (2005). The deterministic part of IPC-4: An overview. Journal of Artificial Intelligence Research, 24, 519-579.

Hoffmann, J., \& Nebel, B. (2001). The FF planning system: Fast plan generation through heuristic search. Journal of Artificial Intelligence Research, 14, 253-302.

Jonsson, P., \& Bäckström, C. (1995). Incremental planning. In New Directions in AI Planning: EWSP'95-3rd European Workshop on Planning, pp. 79-90, Assisi, Italy.

Jonsson, P., \& Bäckström, C. (1998a). State-variable planning under structural restrictions: Algorithms and complexity. Artificial Intelligence, 100(1-2), 125-176.

Jonsson, P., \& Bäckström, C. (1998b). Tractable plan existence does not imply tractable plan generation. Annals of Mathematics and Artificial Intelligence, 22(3-4), 281-296.

Kambhampati, S. (1995). Admissible pruning strategies based on plan minimality for planspace planning. In Proceedings of the 14th International Joint Conference on Artificial Intelligence, pp. 1627-1635, Montreal, Canada.

Katz, M., \& Domshlak, C. (2007). Structural patterns heuristics. In ICAPS-07 Workshop on Heuristics for Domain-independent Planning: Progress, Ideas, Limitations, Challenges, Providence, RI.

Klein, I., Jonsson, P., \& Bäckström, C. (1998). Efficient planning for a miniature assembly line. Artificial Intelligence in Engineering, 13(1), 69-81.

Knoblock, C. (1994). Automatically generating abstractions for planning. Artificial Intelligence, 68(2), 243-302.

Newell, A., \& Simon, H. A. (1963). GPS: A program that simulates human thought. In Feigenbaum, E. A., \& Feldman, J. (Eds.), Computers and Thought, pp. 279-293. Oldenbourg.

Sacerdoti, E. (1974). Planning in a hierarchy of abstraction spaces. Artificial Intelligence, $5,115-135$.

Tenenberg, J. D. (1991). Abstraction in planning. In Allen, J. F., Kautz, H. A., Pelavin, R. N., \& Tenenberg, J. D. (Eds.), Reasoning About Plans, chap. 4, pp. 213-283. Morgan Kaufmann.

Williams, B., \& Nayak, P. (1996). A model-based approach to reactive self-configuring systems. In Proceedings of the 13th National Conference on Artificial Intelligence (AAAI), pp. 971-977, Portland, OR.

Williams, B., \& Nayak, P. (1997). A reactive planner for a model-based executive. In Proceedings of the 15th International Joint Conference on Artificial Intelligence (IJCAI), pp. 1178-1185, Nagoya, Japan. 